\title{
PRICING BARRIER AND BARRIER CRACK OPTION UNDER LEVY PROCESS
}

\author{
by \\ Rofeide Jabbari \\ A thesis \\ presented to Ryerson University \\ in partial fulfilment \\ of the requirements for the Degree of \\ Master of Science \\ in the program of \\ Applied Mathematics
}

Toronto, Ontario, Canada, 2019

(C) Rofeide Jabbari, 2019 
AUTHOR'S DECLARATION FOR ELECTRONIC SUBMISSION OF A THESIS

I hereby declare that I am the sole author of this thesis. This is a true copy of the thesis, including any required final revisions, as accepted by my examiners.

I authorize Ryerson University to lend this thesis to other institutions or individuals for the purpose of scholarly research.

I further authorize Ryerson University to reproduce this thesis by photocopying or by other means, in total or in part, at the request of other institutions or individuals for the purpose of scholarly research.

I understand that my thesis may be made electronically available to the public. 


\title{
PRICING BARRIER AND BARRIER CRACK OPTION UNDER LEVY PROCESS
}

\author{
Master of Science, 2019 \\ Rofeide Jabbari \\ Applied Mathematics \\ Ryerson University
}

In this thesis we study and analyze the pricing of barrier and barrier crack options under a Time-Changed Levy process. Oil and gasoline in Canada are our underlying commodities of interest in this study. To characterize the dynamics of oil and gasoline prices, Black-Scholes and Time-Changed models based on Levy process are proposed. To verify the model, real data of the Canada oil and gas market is used. While the pricing methods based on Monte Carlo are the well-known and dominant for price calculation, we propose a Fourier Transform (FT) for the pricing, which provide some important advantages to the Monte Carlo method such as computation speed without compromising any accuracy. The method is also applied to Crack spread contracts to reduce the risk. 


\section{Acknowledgements}

I would like to gratefully acknowledge all who have supported me to make this dissertation possible. First and foremost, I would like to express my gratitude to my supervisor, Prof. Pablo Olivares, for all his guidance and support. It has been a real privilege to work with such a distinguished professor and great leader.

I also would like to acknowledge Teresa Lee and department of mathematics.

I wish to express my gratitude to my family in Iran, my husband, my parents, my parents' in-law, as well as my brother and sisters for their support and encouragements. Especially, I would like to thank my husband, Mojtaba that has blessed me with her unconditional support and encouragement. 


\section{Dedication}

To my husband

Mojtaba

$\&$

My lovely daughter Jana 


\section{TABLE OF CONTENTS}

Declaration ............................. ii

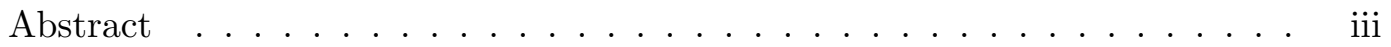

Acknowledgments ......................... iv

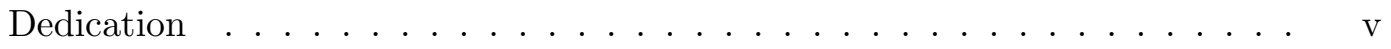

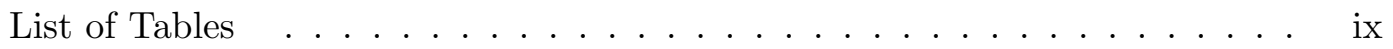

List of Figures . . . . . . . . . . . . . . . .

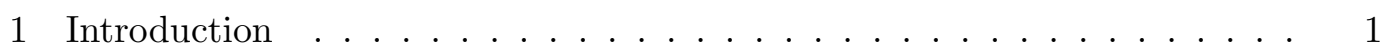

1.1 Energy-Crude Oil and Its Derivatives Market . . . . . . . . . . 1

1.1.1 Basic Definition in Energy Market . . . . . . . . . . . . . 3

1.2 Financial Derivatives . . . . . . . . . . . . . 5

1.2.1 Different Types of Option . . . . . . . . . . . 7

1.2.2 Barrier Options Definition and Pricing . . . . . . . . . 8

1.3 Classic Black-Scholes Model . . . . . . . . . . . . . . . . . . . . 12

1.4 Statistics Analysis of Underlying Assets . . . . . . . . . . . . . . 13

1.5 Levy Process and Time-Changed Models . . . . . . . . . . . . . 21

1.5.1 Normal Inverse Gaussian (NIG) Levy Process . . . . . . . . . 22 
1.6 Monte-Carlo Simulation . . . . . . . . . . . . . . . . . 23

1.7 Option Pricing Using Advanced Transformer Techniques . . . . . . 24

1.8 Thesis Objective and Results . . . . . . . . . . . . . 25

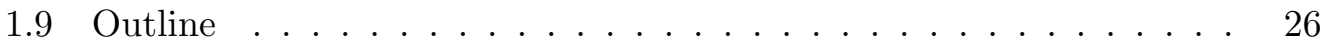

2 Mathematical Basis of Option Pricing . . . . . . . . . . . . . . 28

2.1 Black-Scholes Approach . . . . . . . . . . . . . . . . 28

2.1.1 The Black-Scholes Model Solution . . . . . . . . . . . . 32

2.2 Levy Processes . . . . . . . . . . . . . . . . . . . . . . . 33

2.2.1 Examples of Levy Processes _. . . . . . . . . . . . . 35

2.3 Time-Changed Subordinator _. . . . . . . . . . . . . 36

2.3.1 Inverse Gaussian Subordinator . . . . . . . . . . . . . 36

3 BARRIER PRICING BASED ON CONVENTIONAL BLACK-SCHOLES

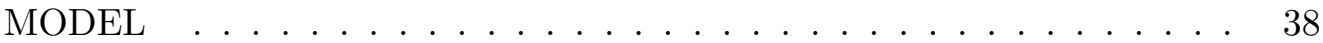

3.1 Barrier Option for Black-Scholes Model . . . . . . . . . . . . . 38

3.1.1 Univariate Black-Scholes Model . . . . . . . . . . . . . . . . . 39

$3.1 .2 \quad$ Empirical Data . . . . . . . . . . . . . . . . . 46

3.2 Pricing Under Univariate Black-Scholes Model . . . . . . . . . . . . 47

3.2.1 Bivariate Black-Scholes Model . . . . . . . . . . . . 55

3.3 Pricing Under Bivariate Black-Scholes Model . . . . . . . . . . . . 58

4 Pricing Barrier Option Based on Time-Changed model by Using Monte Carlo 


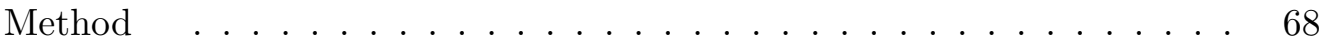

4.1 Time-Changed Model . . . . . . . . . . . . . . . . . . . . 68

4.1.1 Univariate Time-Changed Model . . . . . . . . . . . . 68

4.1.2 Bivariate Time-Changed Model . . . . . . . . . . . . . . 70

4.2 The Monte Carlo Method and Pricing Applications . . . . . . . . . 71

4.3 Algorithm of Monte Carlo Output Functions _ . . . . . . . . 73

4.3.1 Algorithm of Monte Carlo Under Univariate Time-Changed Model . . . . . . . . . . . . . . . . . . . . . . . . . 73

4.3.2 Algorithm of Monte Carlo for Bivariate Time-Changed Model 81

4.4 Monte Carlo Pricing Computation . . . . . . . . . . . . . . . 85

4.4.1 Monte Carlo Pricing under Univariate Time-Changed Model 86

4.4.2 Monte Carlo Pricing under Bivariate Time-Changed Model . 97

4.5 Cosine Fast Fourier Transform Method . . . . . . . . . . . . 108

4.5.1 COS Fast Fourier Transform Method _. . . . . . . . . . 108

4.5.2 Cosine Method in Barrier Option Time Changed . . . . . . . 110

4.5.3 COS Method Algorithm . . . . . . . . . . . . . . . . . . 112

5 Conclusion . . . . . . . . . . . . . . . . . . . . . . 114

References ........................... 115

Appendix . . . . . . . . . . . . . . . . . . . 120

$.1 \quad$ MATLAB Codes . . . . . . . . . . . . . . . . 120 


\section{LIST OF TABLES}

1.1 Different Types of Barrier Option . . . . . . . . . . . . . . 9

1.2 Statistics of Empirical Data _. . . . . . . . . . . 15

1.3 Mean, Variance, Skewness, Kurtosis for NIG . . . . . . . . . . 23

3.1 Prices of In Univariate Barrier Option . . . . . . . . . . . . . . 45

3.2 Prices of Out Univariate Barrier Option. . . . . . . . . . . . . 46

$3.3 \mu$ and $\sigma$ for all three assets . . . . . . . . . . . . . 47

$4.1 \mu$ and $\sigma$ for all three assets in Time-Changed Model . . . . . . . . . 86 


\section{LIST OF FIGURES}

1.1 Barrel of Crude Oil Derivatives Per 2009 Report . . . . . . . . . 2

1.2 Empirical Spot Price, Returns and Log Returns for Brent Oil . . . . 16

1.3 Cumulative Distribution Function from Empirical Data for Brent Oil Price ............................. 17

1.4 Empirical Spot Price, Returns and Log Returns for WTI Oil . . . . 18

1.5 Cumulative Distribution Function from Empirical Data for WTI Oil

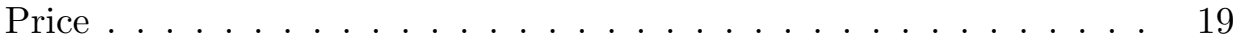

1.6 Empirical Spot Price, Returns and Log Returns for Ontario Gasoline 20

1.7 Cumulative Distribution Function from Empirical Data for Ontario Gasoline Price . . . . . . . . . . . . . . . . . . . . . . . 21

3.1 Differnet types of DIBC when $K>M \ldots \ldots \ldots$. . . . . . 41

3.2 Differnet types of DIBC when $K<M \ldots \ldots$. . . . . . . . . . 43

3.3 Simulation of option price for Brent Oil in Black-Scholes Model . . . 49

3.4 Simulation of option price for WTI Oil in Black-Scholes Model . . . 50

3.5 Simulation of option price for Ontario Gasoline in Black-Scholes Model 51

3.6 Simulation of option price for Brent Oil in Black-Scholes Model . . . 52

3.7 Simulation of option price for WTI Oil in Black-Scholes Model . . . 53

3.8 Simulation of option price for Ontario Gasoline in Black-Scholes Model 54 
3.9 Barrier Option Prices for Two Assets(Brent Oil and Onatrio Gasoline)by using Bivariate Black-Scholes Model . . . . . . . . . . . . 59

3.10 Barrier Option Prices for Two Assets(WTI Oil and Onatrio Gasoline)by using Bivariate Black-Scholes Model . . . . . . . . . . . . . 60

3.11 Barrier Option Prices for Two Assets(Brent and WTI oil)by using Bivariate Black-Scholes Model . . . . . . . . . . . . . . . . . . . 61

3.12 Barrier Option Prices for Two Assets(Brent Oil and Onatrio Gasoline)by using Bivariate Black-Scholes Model . . . . . . . . . . . . . 62

3.13 Barrier Option Prices for Two Assets(WTI Oil and Onatrio Gasoline)by using Bivariate Black-Scholes Model . . . . . . . . . . . . 63

3.14 Barrier Option Prices for Two Assets(Brent and WTI Oil)by using Bivariate Black-Scholes Model . . . . . . . . . . . . . . . . . . 64

3.15 Barrier Option Prices for Two Assets(Brent Oil and Ontario Gasolinel)by using Bivariate Black-Scholes Model . . . . . . . . . . . .

3.16 Barrier Option Prices for Two Assets(WTI Oil and Ontario Gasolinel)by using Bivariate Black-Scholes Model . . . . . . . . . . . . . 66

3.17 Barrier Option Prices for Two Assets(Brent and WTI Oil)by using Bivariate Black-Scholes Model . . . . . . . . . . . . . . . . 67

4.1 Flowchart Depicting the functions created to obtain the Price Option. 73

4.2 Inverse Gaussian Subordinator Simulation Result . . . . . . . . . . . 75

4.3 Brownian Motion Simulation _. . . . . . . . . . . . . . . 77 
4.4 Spot Price for an Asset . . . . . . . . . . . . . . . . 79

4.5 Bivariate Inverse Gaussian Subordinator . . . . . . . . . . . . . . . 82

4.6 Bivariate Brownian Motion Simulation . . . . . . . . . . . . . 84

4.7 Simulation of option price for Brent Oil in Univariate Time-Changed Model .............................. 88

4.8 Simulation of Option Price for WTI Oil in Univariate Time-Changed Model ............................... 89

4.9 Simulation of Option Price for Ontario Gasoline in Univariate TimeChanged Model . . . . . . . . . . . . . . . . . . . . 90

4.10 Simulation of Option Price for Brent Oil in Univariate Time-Changed

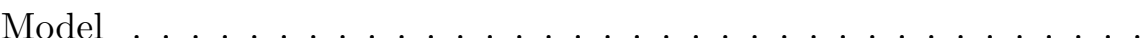

4.11 Simulation of Option Price for Brent Oil in Univariate Time-Changed Model ............................ 92

4.12 Simulation of Option Price for Ontario Gasoline in Univariate TimeChanged Model . . . . . . . . . . . . . . . . . . . . 93

4.13 Simulation of Option Price for Brent Oil in Univariate Time-Changed

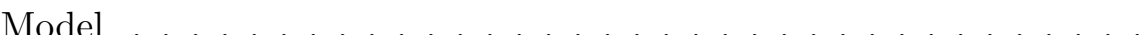

4.14 Simulation of Option Price for WTI Oil in Univariate Time-Changed Model ........................... 95

4.15 Simulation of Option Price for Ontario Gasoline in Univariate TimeChanged Model . . . . . . . . . . . . . . . . . . . . 96 
4.16 Bivariate Simulation of Barrier Option Price for Ontario Gasoline and Brent Oil in Bivariate Time-Changed Model . . . . . . . . . . . . . . 99

4.17 Simulation of Option Price for Ontario Gasoline and Brent Oil in Bivariate Time-Changed Model . . . . . . . . . . . . . . . . 100

4.18 Simulation of Option Price for Ontario Gasoline and Brent Oil in Bivariate Time-Changed Model . . . . . . . . . . . . . . . . . 101

4.19 Bivariate Simulation of Barrier Option Price for Ontario Gasoline and WTI Oil using Time-Changed Model . . . . . . . . . . . . . . . . . . 102

4.20 Simulation of Option Price for Ontario Gasoline and WTI Oil in Bivariate Time-Changed Model . . . . . . . . . . . . . . . 103

4.21 Simulation of Option Price for Ontario Gasoline and WTI Oil in Bivariate Time-Changed Model . . . . . . . . . . . . . . . . . 104

4.22 Simulation of Option Price for Brent and WTI Oil in Bivariate TimeChanged Model . . . . . . . . . . . . . . . . . . . 105

4.23 Simulation of Option Price for Brent and WTI Oil in Bivariate TimeChanged Model . . . . . . . . . . . . . . . . . 106

4.24 Simulation of Option Price for Brent oil and WTI Oil in Bivariate Time-Changed Model . . . . . . . . . . . . . . . . . . 107 


\section{Chapter 1}

\section{INTRODUCTION}

In this chapter we will discuss the energy market and specifically the Oil market. Basic terminology will be explained for introducing energy market.

\subsection{Energy-Crude Oil and Its Derivatives Market}

Any commodity market that is dealing with supply and trade of energy is an energy market. That includes electricity, nuclear energy, renewable energy, coal, crude oil and its derivatives and natural gas. Energy markets have a key role in global economy especially crude oil. Consequently, studying and analyzing the crude oil energy market is vital to global economy's stability. In fact, crude oil is the most traded commodity in the world and every energy trader needs to have a solid understanding of its market. Accordingly, economists and finance experts, have generated some financial tools trying to predict, control or mitigate the risk of uncertainty and volatility in the crude oil and its derivative markets. Any negligence of the risk involved in the crude oil market could cause a great risk to the financial flow of a lot of companies and countries including Canada. Crude oil price fluctuation and price shock could put companies in bankruptcy if they have not prepared themself for risk control by utilizing right financial tools and hedging their funds against the 
risk. Even though a lot of tools have been introduced and new mathematical and numerical methods have been applied to tame and predict the crude-oil market, because of large uncertainly involve, it is still the most challenging markets in the finance.

Figure 1.1: Barrel of Crude Oil Derivatives Per 2009 Report

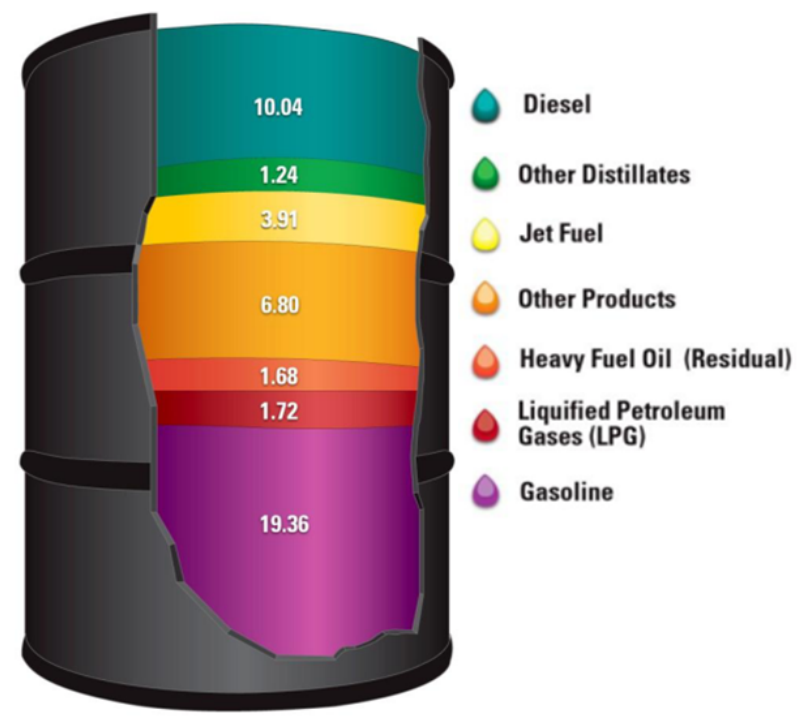

Figure (1.1) is retrieved from https://www.energy.gov/articles/hows-and-whysreplacing-whole-barrel.

Financial tools such as forwards, futures and options have been introduced through appropriate hedging strategies to manage the risk in the very volatile energy market and generally for all types of market. However, if these tools are misused and the risk is overestimated or underestimated, they will do more damage than good by pushing companies to overspending and losing competitiveness or neglecting the risk 
and losing their fund and capital.

\subsubsection{Basic Definition in Energy Market}

In a Spot Market, commodities are delivered on the day of the transaction. Consumers are usually purchasing small quantities of a commodity immediate use in spot transactions.(Edvards,2010)

Commodities deliver immediately in spot market at the same day of purchasing. When commodities are schedule for delivery at some agreed days the future, this is called Forward Market.

Buyers and sellers allow to agree on transactions ahead of time in the forward trading market. By real contracts, this gives both sides enough time to prepare for delivery or receipt of a physical commodity.

Trades in the forward market are generally specified by several factors:

- Underlying Instrument. The commodity being traded, usually with a description of minimum quality standards that must be met.

- Quantity. The amount of the commodity that must be delivered.

-Delivery Price. The price per unit due at delivery.

- Delivery Date. The date at which the underlying instrument will be delivered.

- Delivery Location. The location at which the underlying instrument will be delivered. (Edvards,2010)

A trade is a transaction and a position is the pure presentation that comes from 
one or more trades. If a buyer makes more than one transaction to buy for example crude oil, the combination of all of those trades is known as a position.

We have long and short positions in the market by the term of trading. In the long position, the trader will benefit from a rise in the price of the commodity. When trader make a benefit from fall in the price of a commodity, it is called short position.

\section{OVER THE COUNTER AND EXCHANGES}

Traders can sell or buy energy product in two ways, when it trade directly between two parties, we call it over the counter $(O T C)$. Another way is exchange, that trades to be made through an intermediary (Schoutens,2003). In over the counter trades, a contract for trading involves signing each and every time a trade is made. If one party goes bankrupt, the other party usually has no option other than going through bankruptcy proceeding. Accordingly, each party is responsible for checking the other party reliability.

When trades are made through an exchange, they are called exchange traded transactions. There are some difficulties in associating with direct contract, then just limited people can enter into OTC trades. Exchange make the market more accessible to people, where parties instead of transacting with each other, they trade through exchange. As the result, the exchange trade will be easier and the risk of the OTC will be eliminated.

Cash trades, futures, and forwards are of the three most common types of energy trades. Cash trades are an exchange of physical commodity for cash in spot market. 


\section{Crack and Spark Spreads}

Spreads are trading strategies that include more than one financial asset. Crack spread is one of popular trading strategy where various derivatives are combined.

For example refiner who buys crude oil and sells oil product is more interested in price difference between the crude oil and the products than each of them individually. Same concept can be applied into future trading when a hedger can buy crude futures and sell product future. However, in reality it is not practical to account all the barrel's product and crack spread will ignore a large share of the barrel. Typically, 3-2 rule will be applied when 3 barrel of crude future will be traded for two barrels of gasoline and one barrel of heating oil.(Dahl,2015)

\subsection{Financial Derivatives}

Financial experts have introduced some techniques and tools to control the risk of the market fluctuations called financial derivatives. Forward, future and option contracts are among useful tools to mitigate the risk and alleviate the market fluctuation.

Forward Contracts is an agreement to buy/sell a fixed amount of an asset at a given price at a certain time in the future $($ Hull,2015). Forward contract delivery is mandatory and is traded in the OTC market between two financial institutions (Hull,2015). 
Future Contracts like a forward contracts is an agreement to buy/sell a commodities at a future time for a certain price. Futures contract is traded on an exchange while forward contracts will be traded in the OTC market (Hull,2015).

Besides where they are traded Margin is the most important difference between futures and forwards market. It is a deposit used in the futures trades that required by the exchange to ensure that traders meet their obligations. There is no margin in forward market.

\section{Option Contracts}

Another alternative financial derivative to forward and future contracts are options by which its owner or holder has the right, not the obligation, to buy or sell an asset at a specified price within a specified time (Zhang,1998). The exercise price of the option contract is called strike or exercise price and the date in the contract is called maturity or expiration time. The option can be traded either at exchange or OTC markets.

Among financial derivatives, option contracts are the most popular one. While, based on data from Chicago Board Option Exchange (CBOE), it looks like that only $10 \%$ of the option contracts are exercised and $90 \%$ of them are useless. There is an argument that option contract are useless. However, in reality around $55-60 \%$

of these option contracts will be closed before the expiry. It means that more than $65-70 \%$ of option contract will be used to balance the market and only $30-35 \%$ of them expired worthless. 


\subsubsection{Different Types of Option}

If the owner can only exercise their right to buy/sell their at maturity time, the option is called a European option. An American option, is the option that the owner can exercise their right to buy/sell at any time before or at expiration or maturity time, unlike European option which cannot be exercised before maturity time. The holder of the option needs to pay a non-refundable premium money to the seller when they buy the option (Zhang,1998). The option that gives the right to holder to buy an underlying asset is called call option and the option that gives the holder the right to sell the option is called put option (Zhang,1998). In call option contracts, the holder makes a profit if the asset price rises above the strike price. In put option contracts, the holder gains if the strike price is higher than the asset price.

One of the European and American options drawback is that they do not provide any information about the price trajectory, specially European option as it is very important factor to settle the option price at the time of exercise. Therefore, different types of path-dependent option such as Asian option, barrier option, look back option have been introduced(Zhang,1998). Among them the barrier options which are the focus of this thesis also is one of the most demanded derivative because of the flexibility and better price it offers to users. 


\subsubsection{Barrier Options Definition and Pricing}

Barrier Options are options in which the right of the holder to buy or sell the stock by the end of the contract is conditional on the stock crossing( or not crossing) a certain barrier before the expiry date. (Cont \& Tankov,2004)

The barrier option can be divided in two main categories Knock-in and Knock-out. A Knock-in barrier option becomes effective only if the underlying asset first reaches a certain barrier level.

Oppositely, a Knock-out barrier option immediately terminates if the underlying asset reaches a certain barrier level. (Marie,2014)

Depending on how to reach the barrier price from above or below, you can categorize the barrier options to up-and-knock-in, up-and-knock-out, down-and-knock-in and down-and-knock-out options for both call and put options (Zhang,1998). 


\begin{tabular}{|c|c|c|}
\hline Types & & Option Price \\
\hline \hline \multirow{2}{*}{ Call } & Knock-In & Down \\
& Knock-Out & Down \\
& & Up \\
\hline \multirow{2}{*}{ Put } & Knock-In & Down \\
& & Up \\
& Knock-Out & Down \\
& & Up \\
\hline
\end{tabular}

Table 1.1: Different Types of Barrier Option

In knock-in and knock-out options, the seller has the opportunity to cancel it before it expiry or termination time. This helps the seller to reduce their risk. Therefore, to attract the buyer, they offer more appealing price on these types of options. We denote $M_{t}^{S}, m_{t}^{S}$ as

$$
M_{t}^{S}=\sup \left\{S_{t} ; 0 \leq t \leq T\right\} \quad m_{t}^{S}=\inf \left\{S_{t} ; 0 \leq t \leq T\right\}, \quad 0 \leq t \leq T
$$

Where $\mathrm{M}$ is barrier, $\mathrm{K}$ is strike price, $\mathrm{r}$ is interest rate, $\mathrm{T}$ is maturity time and $\mathbf{1}_{\left\{m_{T}^{s}>M\right\}}$ is indicator function.

For single-barrier options, the price of different types of barrier options will be 
defined as below.

Down-and-out barrier call is worthless unless its minimum remains above some low barrier $\mathrm{M}$, in which case it will be treated as a nominal call option. Its at time $T=0$ price is given by

$$
D O B C=\exp (-r T) \mathbb{E}_{Q}\left[\left(S_{T}-K\right)^{+} \mathbf{1}_{\left\{m_{T}^{s}>M\right\}}\right]
$$

Down-and-in barrier call is a standard call with strike $\mathrm{K}$ if its minimum goes below M. If $\mathrm{M}$ is never reached during the lifetime of the option, the option is worthless. Its initial price is given by

$$
D I B C=\exp (-r T) \mathbb{E}_{Q}\left[\left(S_{T}-K\right)^{+} \mathbf{1}_{\left\{m_{T}^{s} \leq M\right\}}\right] .
$$

Up-and-in barrier call is worthless unless its maximum hits some high barrier M, in which case it retains the structure of a standard call with strike K. Its price is given by

$$
U I B C=\exp (-r T) \mathbb{E}_{Q}\left[\left(S_{T}-K\right)^{+} \mathbf{1}_{\left\{M_{T}^{s} \geq M\right\}}\right]
$$

Up-and-out barrier call is worthless unless its maximum is still below the barrier M. Its price is given by

$$
U O B C=\exp (-r T) \mathbb{E}_{Q}\left[\left(S_{T}-K\right)^{+} \mathbf{1}_{\left\{M_{T}^{s}<M\right\}}\right]
$$

Down-and-out barrier put option, it is worthless in the same condition as downand-in barrier call option and its price is given by

$$
D O B P=\exp (-r T) \mathbb{E}_{Q}\left[\left(K-S_{T}\right)^{+} \mathbf{1}_{\left\{m_{T}^{s}>M\right\}}\right]
$$


Down-and-in barrier put option, the price can be found from

$$
D I B P=\exp (-r T) \mathbb{E}_{Q}\left[\left(K-S_{T}\right)^{+} \mathbf{1}_{\left\{m_{T}^{s} \leq M\right\}}\right]
$$

Up-and-in barrier Put option, its price comes from

$$
U I B P=\exp (-r T) \mathbb{E}_{Q}\left[\left(K-S_{T}\right)^{+} \mathbf{1}_{\left\{M_{T}^{s} \geq M\right\}}\right]
$$

Up-and-out barrier Put is worthless if the asset falls to the barrier M, otherwise its price can be calculated by

$$
U O B P=\exp (-r T) \mathbb{E}_{Q}\left[\left(K-S_{T}\right)^{+} \mathbf{1}_{\left\{M_{T}^{s}<M\right\}}\right]
$$

Up-and-out call (put) is a common example of barrier options and usually purchased by an energy producer to hedge their natural long position in the energy markets. Up-and-out call (put), while it is less expensive, provide the same price protection if the price goes down from current level. However, if the price goes up, the risk protection for price drop will be reduced due to the increase in the underlying commodity's price. If the price moves up to pass the barrier price, then the option will be terminated.

In that case the owner may hedge another call option at a higher strike price to have better protection than the lower strike price that was already canceled. 


\subsection{Classic Black-Scholes Model}

Determining the fair and reasonable price for an option is the main objective and at the same time most the challenging issue of the option market. The model introduced by Fisher Black and Myron Scholes in 1973 was a major breakthrough for option pricing. In the same year Robert Merton presented a similar model by different approach (Merton,1973). The impact of Black-Scholes-Merton (or BlackScholes) model on the option and other financial derivate was so huge that brought Morton and Scholes a Nobel prize for economics in 1997. Black was passed away at 1995, otherwise he definitely would be one of the prize recipient.

Skipping all mathematical details of the model, if we have $\mathrm{S}$ as underlying asset price, $\mathrm{K}$ as strike price, $\mathrm{T}$ as time to expiration or maturity time, $\mathrm{r}$ as risk free interest rate and $\sigma$ as volatility of underlying price we can extract two separate formulas to price of call and put options. Black-Scholes model assumes the underlying asset price develops according to geometric Brownian motion (named after R. Brown)also known as Gaussian Wiener process as presented below(Schoutens,2003)

$$
d S_{t}=S_{t}\left(\mu d t+\sigma d B_{t}\right), \quad S_{0}>0 .
$$

where $B_{t}$ is a standard Gauss-Wiener process (Brownian motion), $\mu$ is the mean and $\sigma$ is standard deviation or as often called volatility of the underlying asset price(Zhang,1998). Brownian motion is in fact the dynamic counterpart of its static Normal distribution equivalent. Solving the equation above by Ito formula will lead 
to following formula

$$
S_{t}=S_{0} \exp \left(\left(\mu-\frac{1}{2} \sigma^{2}\right) t+\sigma B_{t}\right)
$$

or

$$
\log \left(\frac{S_{t}}{S_{0}}\right)=\left(\mu-\frac{1}{2} \sigma^{2}\right) t+\sigma B_{t}
$$

From (1.2), it shows the returns in a Black-Scholes model is log-normally distributed as $B_{t}$ has normal distribution wither zero mean and variance $\sigma$ (Zhang,1998).

To reach to the (1.2) formula the Black and Scholes model assumes:

1. Interest rate is constant through the time.

2. Volatility of the price remains constant over the time.

3. Price follow the geometric Brownian motion (Wiener process).

4. The price changes are log-normally distributed.

Among these assumption, considering the volatility of the price constant is far from reality, as by analyzing a real price data we can easily see that, this assumption is not accurate.

\subsection{Statistics Analysis of Underlying Assets}

For financial engineers and experts, statistical analysis of stocks or option of the underlying assets is critical where parameters such as mean, variance, skewness and kurtosis have always been utilized. For any X random data, mean or average will 
be defined as follow

$$
\mu=\mathbb{E}[X]
$$

Variance which shows the deviation of the data from the average is defined as below

$$
\sigma^{2}=\mathbb{E}\left[(X-\mu)^{2}\right]
$$

Square root of variance or $\sigma$ is called deviation.

Skewness measures the degree to which a distribution is asymmetric:

$$
\text { Skewness }=\frac{\mathbb{E}\left[(X-\mu)^{3}\right]}{\sigma^{3}}
$$

For a normal distribution, the skewness is zero, while in real world, the stock or option prices are not zero sometimes far from zero skewness mostly negative showing has longer tail to the left (Schouten).

Kurtosis or fat tail, is a parameter that shows if any the large movement has happened in the data like jumping that we can see happen more often in finance derivative markets.

$$
\text { Kurtosis }=\frac{\mathbb{E}\left[(X-\mu)^{4}\right]}{\sigma^{4}}
$$

For example the Normal distribution is the kurtosis is 3. In the following table, mean, variance, skewness and kurtosis are presented for Brent and WTI Oil and Ontario Gasoline from empirical data. It shows that a empirical data has bigger kurtosis than 3 , suggesting that in real word market is keen to have price jumping in contrast to Normal distribution and Balck-Scholes model presumptions. (Schoutens). 


\begin{tabular}{ccccc}
\hline Series & Mean & Standard Deviation & Skewness & Kurtosis \\
\hline \hline BRE Oil & 0 & 0.0202 & 0.3937 & 2.9560 \\
WTI Oill & 0 & 0.0223 & 0.1990 & 3.0553 \\
Ontario Gasoline & 0.0131 & 0.0185 & -1.1683 & 21.0090
\end{tabular}

Table 1.2: Statistics of Empirical Data

Unlike Black-Scholes assumption explained in previous section asset prices jump, leading to non-normal return distribution and also return volatility varies scholastically over time which Black-Scholes cannot predict very accurately. Time-Changed models based on Levy processes have been introduced to make the model more consistent and robust with observed prices in the market (Carr and Liuren,2003). 
Figure 1.2: Empirical Spot Price, Returns and Log Returns for Brent Oil
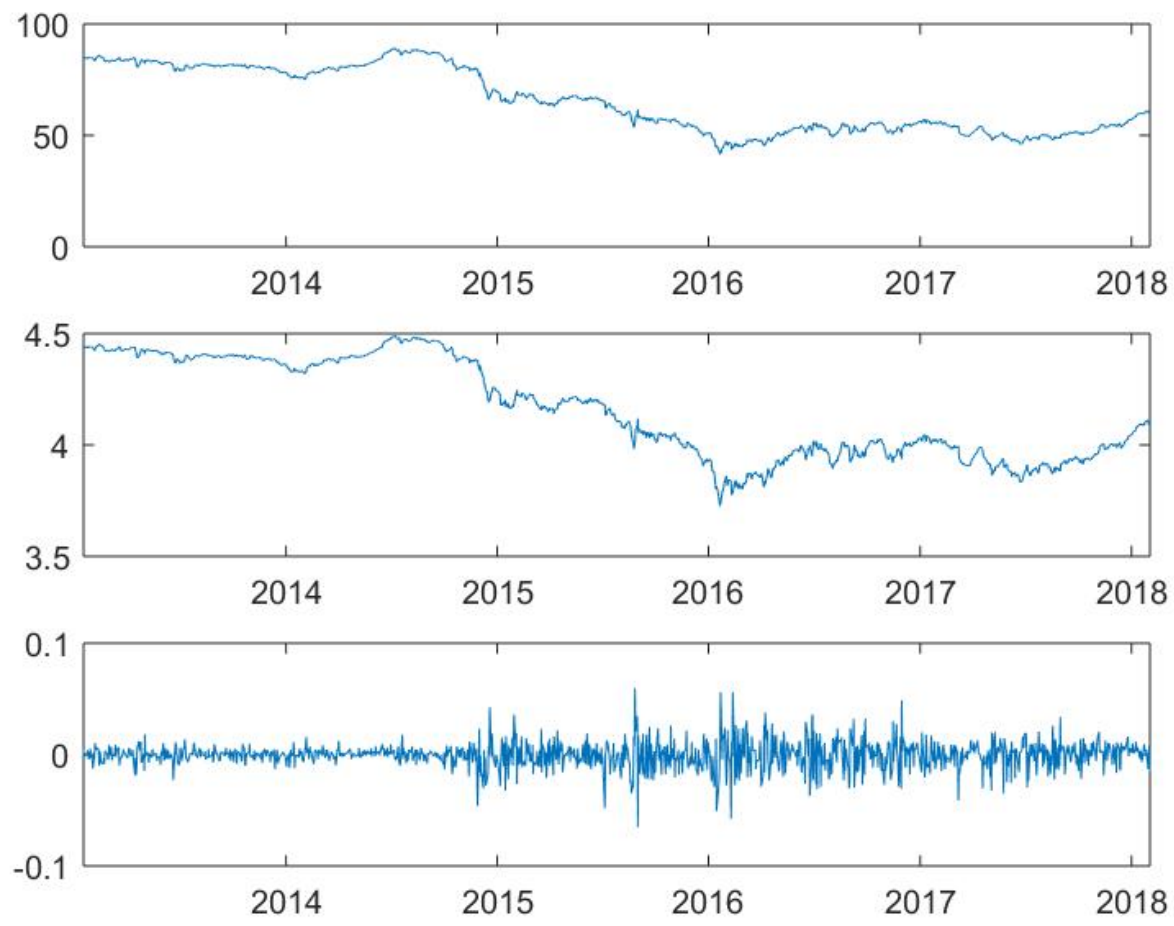

The graphs above show the data for Brent oil prices the 5 years empirical data April 2014 -March 2019. The graph at the top shows the spot price for Brent oil. The second graph shows the returns for same commodity. The third graph illustrates the Brent oil's log-returns. A gradual decrease in combination with jumps and steadiness is observed from graphs. 
Figure 1.3: Cumulative Distribution Function from Empirical Data for Brent Oil Price

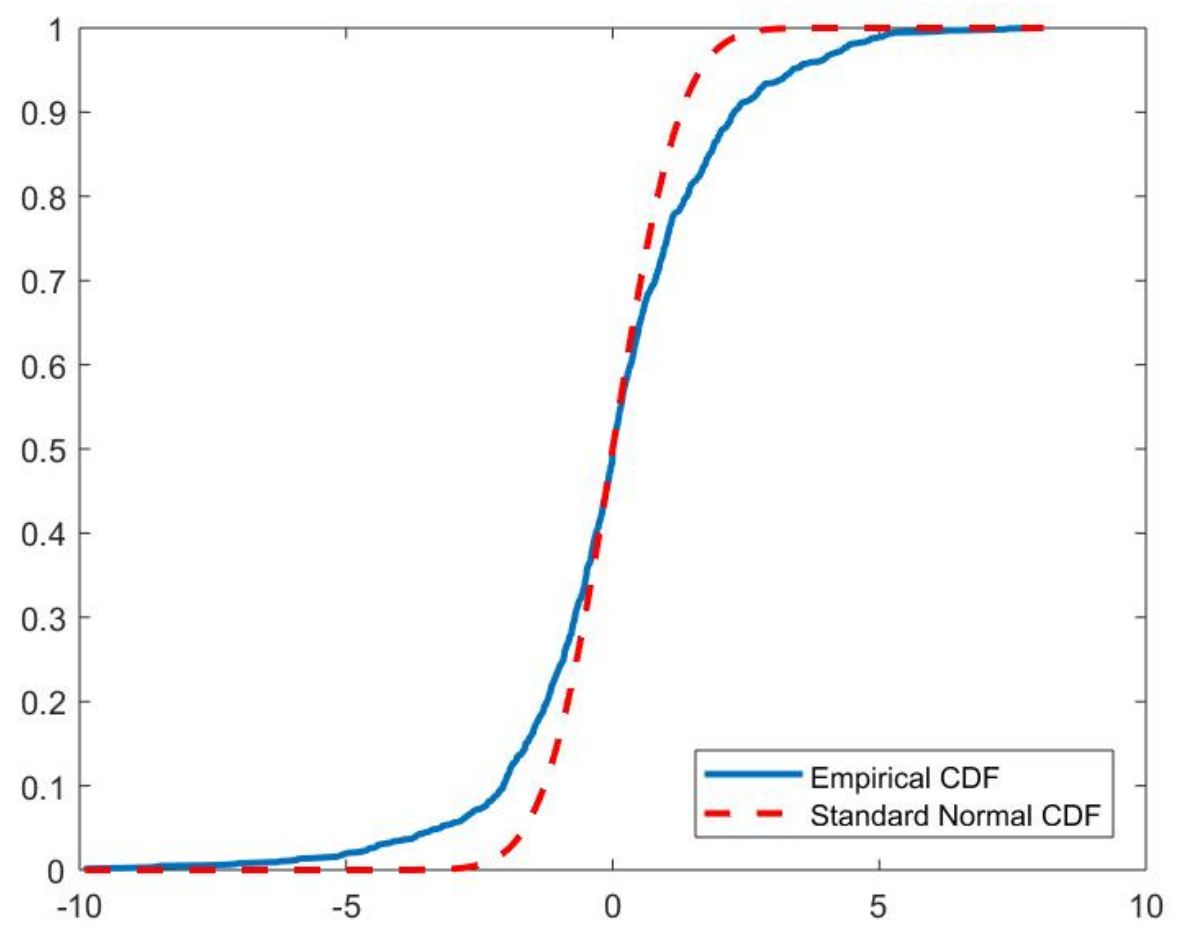

The graph show the CDF for daily empirical data for Brent Oil. Comparing CDF Brent Oil with CDF of Normal distribution shows the WTI Oil does not have a Normal Distribution. 
Figure 1.4: Empirical Spot Price, Returns and Log Returns for WTI Oil
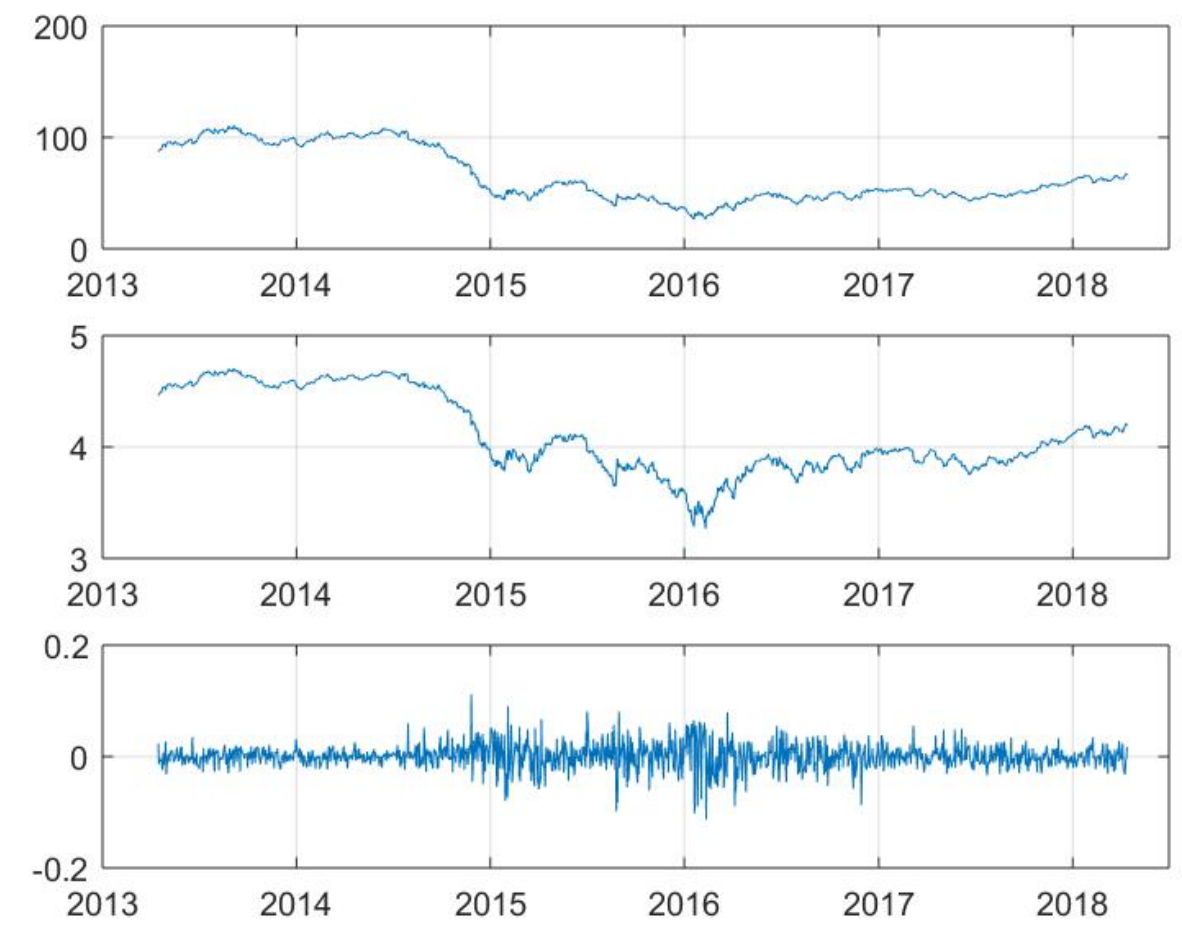

The graphs above show the data for WTI oil prices the 5 years empirical data April 2014 -March 2019. The graph at the top shows the spot price for WTI oil. The second graph shows the returns for same commodity. The third graph illustrates the WTI oil's log-returns. A gradual decrease in combination with jumps and steadiness is observed from graphs. 
Figure 1.5: Cumulative Distribution Function from Empirical Data for WTI Oil Price

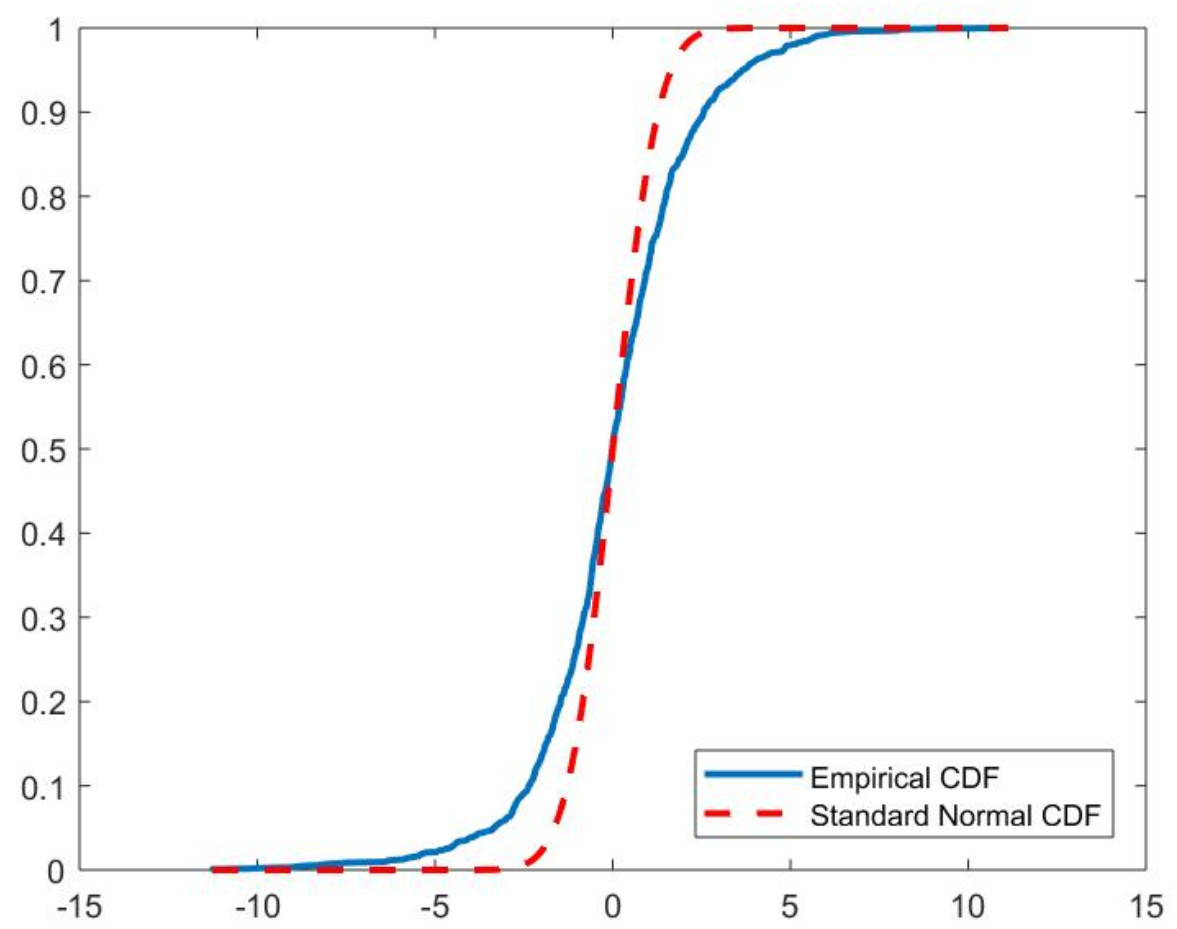

The graph show the CDF for daily empirical data for WTI Oil. Comparing CDF Brent Oil with CDF of Normal distribution shows the WTI Oil does not have a Normal Distribution. 
Figure 1.6: Empirical Spot Price, Returns and Log Returns for Ontario Gasoline
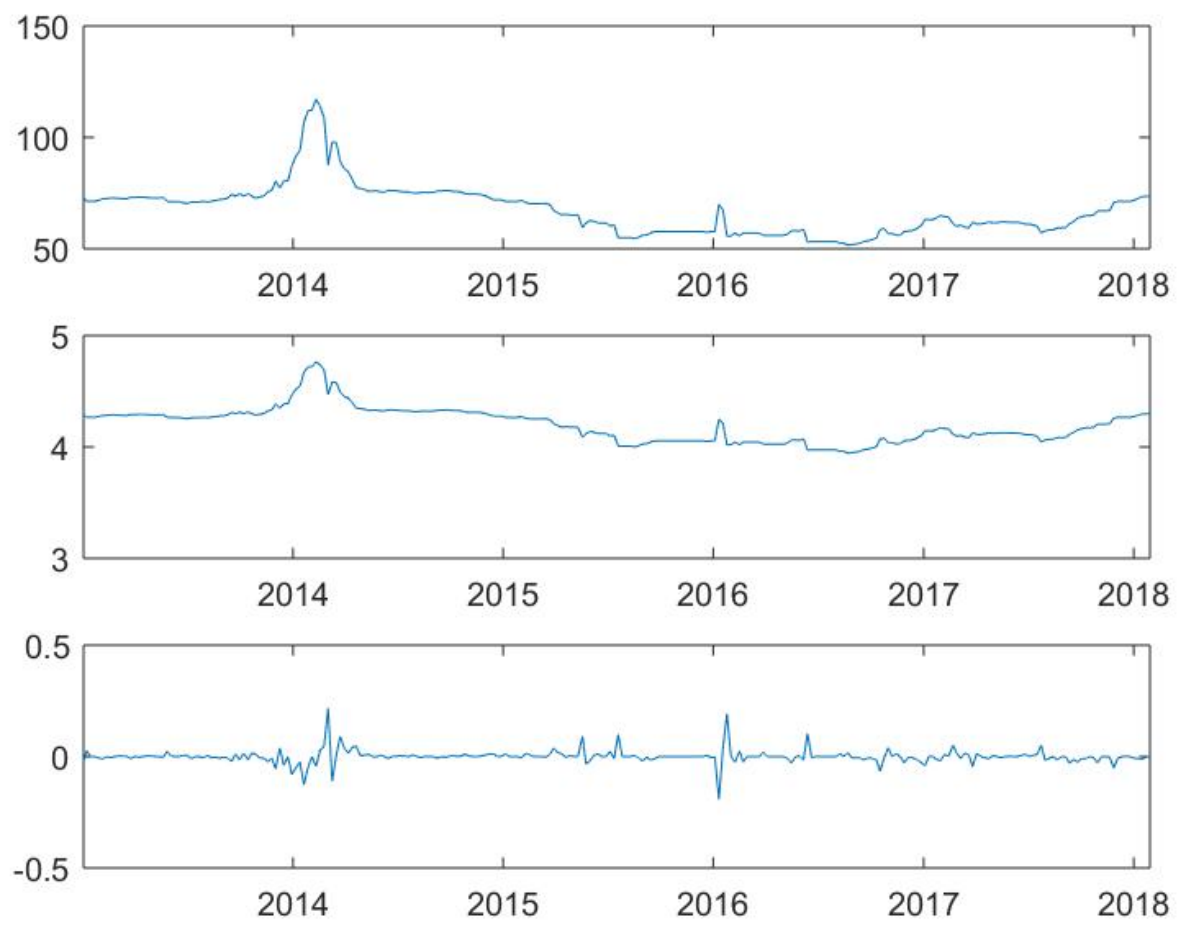

The graphs above show the weekly pricing data for the Ontario gasoline from the 5 years empirical data. The first graph demonstrates the spot price for Ontario gasoline. The second graph shows the returns for same commodity. The third graph illustrates the Ontario gasoline's log-returns. The price increase with high jumps in the first year. After 2014, the price reduce gradually with jumps. 
Figure 1.7: Cumulative Distribution Function from Empirical Data for Ontario Gasoline Price

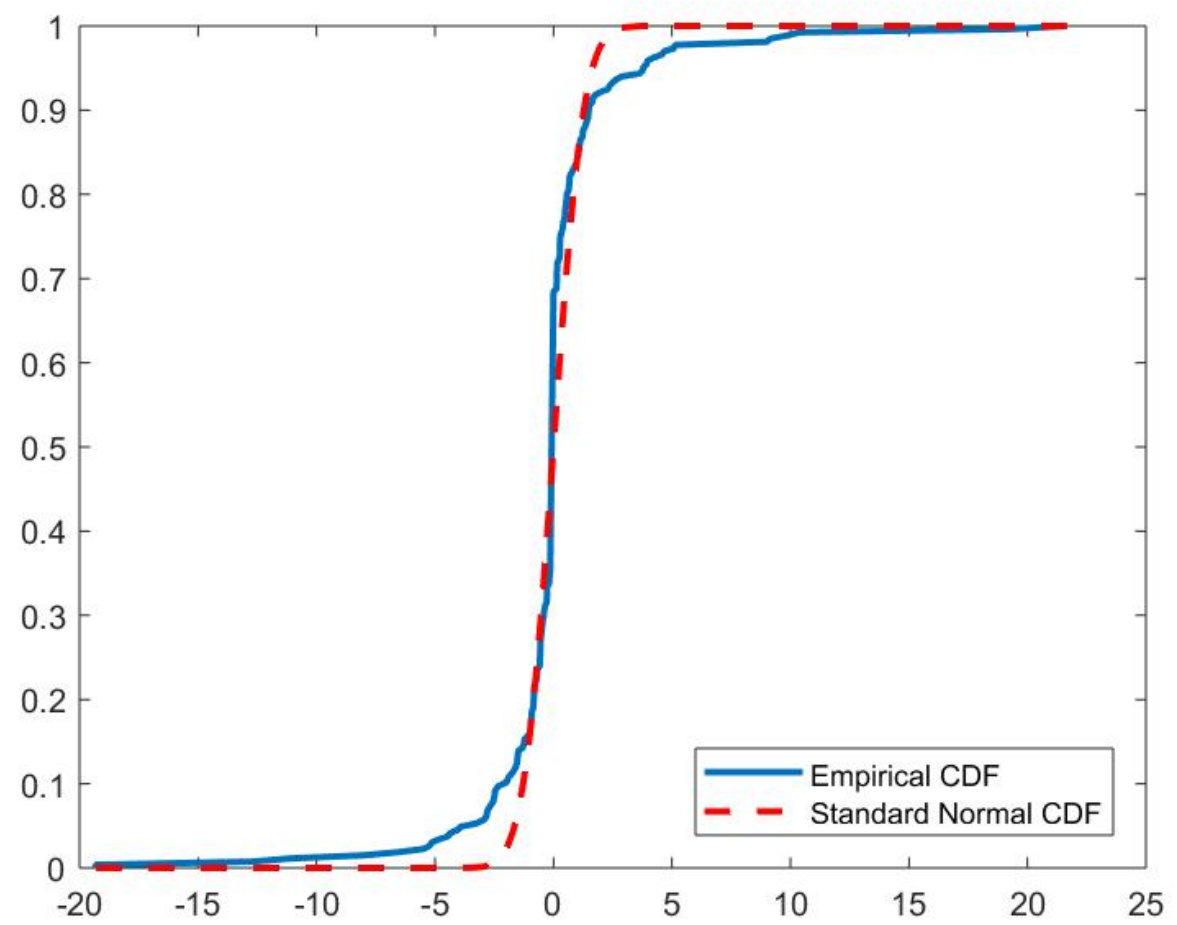

The graph show the CDF for weekly empirical data for Ontario Gasoline. Comparing CDF Ontario Gasoline with CDF of Normal distribution shows the Ontario Gasoline does not have a Normal Distribution.

\subsection{Levy Process and Time-Changed Models}

Looking at (1.1) of the Black-Scholes model and its Brownian motion, we would like to have a similar yet independent and stationary increments process, with a 
more general than the Normal distribution. However, in order to define such a stochastic process the distribution has to be infinitely divisible. Such processes are called Levy processes, in honor of Paul Levy, the pioneer of the theory (Schoutens). Examples of such distribution which can take skewness and excess kurtosis into account are the Variance Gamma (VG), the Normal Inverse Gaussian (NIG), the CGMY, the (Generalized) Hyperbolic Model and the Meixner distributions. All of these techniques are based on Levy process and have their own advantages and disadvantages. In this thesis our focus will be on NIG Levy process which was first proposed by Barndorff-Nielsen at 1995 (Schoutens.W, 2003).

\subsubsection{Normal Inverse Gaussian (NIG) Levy Process}

NIG distribution as a member of Levy process family provides a good flexibility to calibrate its mean, variance, skewness and kurtosis according to empirical data making it very appealing for modeling financial derivatives like option.

The NIG distribution has the characteristic function as follow

$$
\varphi_{N I G}(u ; \alpha, \beta, \delta)=\exp \left(-\delta\left(\sqrt{\alpha^{2}-(\beta+i u)^{2}}-\sqrt{\alpha^{2}-\beta^{2}}\right)\right)
$$

where $\alpha>0,-\alpha<\beta<\alpha$ and $\delta>0$. The mean, variance, skewness and kurtosis of this model will be as follow: 


\begin{tabular}{|c|c|}
\hline Mean & $\frac{\alpha \beta}{\sqrt{\alpha^{2}-\beta^{2}}}$ \\
\hline Variance & $\frac{\alpha^{2} \delta}{\sqrt{\left(\alpha^{2}-\beta^{2}\right)^{3}}}$ \\
\hline Skewness & $\frac{3 \beta}{\alpha \sqrt{\delta} \sqrt[4]{\alpha^{2}-\beta^{2}}}$ \\
\hline Kurtosis & $3\left(1+\frac{\alpha^{2}+4 \beta^{2}}{\alpha^{2} \delta \sqrt{\left(\alpha^{2}-\beta^{2}\right)}}\right)$ \\
\hline
\end{tabular}

Table 1.3: Mean, Variance, Skewness, Kurtosis for NIG

Inverse Gaussian (IG(a, b)) will be defined as a sub-category of NIG by defining $a=1$ and $b=\delta \sqrt{\alpha^{2}-\beta^{2}}$.

\subsection{Monte-Carlo Simulation}

Along introducing new methods to address Black-Scholes model shortcoming like time-changing volatility, numerical techniques are also developed simultaneously to provide solutions for these new techniques mostly two or higher order partial differential equations (PDE) which are not easy to solve mathematically. Two most well-known numerical techniques are Monte-Carlo and binomial option pricing techniques. Numerical methods can provide very accurate response by increasing the steps of simulation even when there more than one uncertainty in the model like time-varying average, time-varying volatility or two underlying assets of course with expense of the processing time. However, with new powerful processor these techniques are becoming more and more appealing. 
Among these techniques, Monte-Carlo technique is the most popular one. IT basically follows with these steps:

1. Generate random number for possible price path based on probability distribution using simulation.

2. Use parameters like volatility and expiry time values to calculate the stock price.

3. Use the stock price at the time of expiry to calculate the option.

4. Repeat steps 1-3 for numerous times and average the results to find the final option price.

\subsection{Option Pricing Using Advanced Transformer Techniques}

The new advanced techniques have been proposed based on convolution and Fast Fourier Transform (FFT) (Carr and Madan,1999). The key idea is to recognize that the usual risk-neutral valuation formula can be calculated as a convolution. This feature, is highly useful, since convolutions in the time domain can be translated easily to the Fourier domain, enabling one to apply the FFT and benefit from its computational power. This recent pricing method, proposed by Lord et al. (2008), was dubbed the convolution method, and is applicable to a wide variety of payoffs requiring only the knowledge of the characteristic function of the model. As such, the method is applicable within many regular affine models, among which is the class of exponential Levy models. Assuming that the characteristic function of the log-stock 
price is known analytically, the price of plain vanilla options can be determined using the Fast Fourier Transform (FFT) method first presented in (Carr,1999). Using this approach, the call price is expressed in terms of an inverse Fourier transform of the characteristic function of the log-stock price under the assumed stochastic process. The resulting formula can then be re-formulated to enable computation using the FFT algorithm that significantly decreases computation time compared to standard numerical methods such as the discrete Fourier transform.

Fourier transform methods are shown to be an effective approach to pricing an option whose underlying asset price process is a Levy process. Instead of applying the direct discounted expectation approach of computing the expectation integral that involves the product of the terminal payoff and the density function of the Levy process, it may be easier to compute the integral of their Fourier transform since the characteristic function (Fourier transform of the density function) of the Levy process is easier to be handled than the density function itself. Actually, one may choose a Levy process by specifying the characteristic function since the LevyKhinchine formula allows a Levy process to be fully described by the characteristic function (Kienitz, Wetterau,2012).

\subsection{Thesis Objective and Results}

In this thesis, we investigate the barrier option pricing for single asset and two highly correlated assets scenario. The analysis started with Black-Scholes model where its 
short coming make it impossible to offer accurate pricing in a very volatile energy market.

Accordingly, the novel single/two asset(s) Time-Changed model was proposed under Levy Process, where we were able to capture essential aspects of the price dynamic including time-changing mean and volatility and seasonal sensitivity, jumps and diffusion. While the proposed models proved to have more realistic estimation the price that should be paid to is computing complexity and time especially where the accuracy of Monte-Carlo algorithm used for these new models directly depends on the number of repetition(here we use one million times). Which my take couple of days for one simulation run using MATLAB.

Accordingly, simultaneous data logging was implemented in software to log the results as soon as simulation data is available.

\subsection{Outline}

In the next chapter, a background of the models utilized in this thesis are provided. Concepts and models like Brownian motion, Black-Scholes model and Levy process are provided in more mathematically depth elaboration. In chapter three, BlackScholes model is presented for both single asset (univariate) and two asset (bivariate) schemes where for bivariate schemes the effect of correlation between two assets price modeling is explained for focusing on crude Oil and Ontario Gasoline as two objective assets. In the chapter four, the real data of the crude oil and gas prices 
are analyzed and it is shown that assuming the constant volatility for these assets is not very realistic, the main assumption that Black-Scholes model is based on. Normal Inverse Gaussian model based on Levy process, one of the most well-known Time-Changing is described in more details. Both univariate and bivariate analysis on crude Oil and Gas along simulation data is provided. The results are compared with results from conventional Black-Scholes model from chapter three. In the last section, Fourier expansions is proposed as solution to overcome the long time consumption issue of the time-Changed model. At the end we will have conclusion. All simulation are supported with MATLAB codes. 


\section{Chapter 2}

\section{MATHEMATICAL BASIS OF OPTION PRICING}

To calculate the price barrier option accurately, having a comprehensive mathematically well developed model that support all types of assets with fix or varying mean and low or high volatility is necessary. Generally models can be divided in to two main categories time-fixed model better known as Black-Scholes model and more realistic Time-Changed model where all parameters of the model can be function of time.

In this chapter, the mathematical background of Black-Scholes model is provided. The Levy process mathematical theory as foundation of Time-Changed models is introduced. The Brownian motion process and the Poisson process ( two subclasses of Levy process) are introduced. At the end of this chapter Levy process subordinator is also described with definition of Inverse Gaussian Random process which will be the Time-Changed technique used in this thesis.

\subsection{Black-Scholes Approach}

Definition 2.1. Let $(\Omega, \mathbb{F}, P)$ be a probability space, an increasing family of $\sigma$ algebra $(\mathbb{F})_{t \in[0, T]}$ called filtration on this space such that

$$
\mathbb{F}_{s} \subseteq \mathbb{F}_{t} \subseteq \mathbb{F} \quad \text { for } \quad 0 \leq s \leq t \leq T
$$


The Brownian motion is the dynamic counterpart of the Normal distribution. Both arise from the Central Limit theorem. Intuitively, it tells us that the suitably normalized sum of many small independent random variables is approximately normally distributed. These results explain the ubiquity of the Normal distribution in a static context. If we work in a dynamic setting, i.e with stochastic processes, Brownian motion appears in a similar way.(Bachelier)

Definition 2.2. A stochastic process $B=\left\{B_{t}, t \geq 0\right\}$ is a standard Brownian motion on some stochastic basis $(\Omega, \mathbb{F}, P)$ if:

(i) $B_{0}=0$

(ii) B has independent increments, (iii)B has stationary increments,

(iv) $B_{t+s}-B_{t}$ is Normally distributed with mean 0 and $\sigma>0$,i.e. $B_{t+s}-B_{t} \simeq N(0, \sigma)$.

Some of important properties of Brownian motion are listed below 1. Martingale Property:

Brownian motion is one of the simplest examples of a martingale.

We have, for all $0 \leq s \leq t$,

$$
\mathbb{E}\left[B_{t} \mid F_{s}\right]=\mathbb{E}\left[B_{t} \mid B_{s}\right]=B_{s} .
$$


The property follows from the instructure of the increments and we have

$$
\mathbb{E}\left[B_{t} \mid B_{s}\right]=\min \{t, s\}
$$

\section{Path Properties:}

It can be proved that Brownian motion has continuous paths, i.e. for any $w \in \Omega$, $B_{t}(w)$ is a continuous function of $t \in \mathbb{R}^{+}$. However, the paths of Brownian motion are very erratic and for example nowhere differentiable.

3. Oscillation Property: Another property is that for a Brownian motion $B=$ $\left\{B_{t}, t \geq 0\right\}$, we have that

$$
P\left(\sup _{t \geq 0} B_{t}=+\infty \quad \text { and } \quad \text { inf }_{t \geq 0} B_{t}=-\infty\right)=1 .
$$

Which means that the Brownian path will keep oscillating between positive and negative values.(Shoutens)

Now by definition Brownian motion we can introduce one of the most important stochastic process in finance, that is relative to Brownian motion: geometric Brownian motion.(Black and Scholes)

To model the time evolution of stock prices in the Black-Scholes model consider the change the price $\Delta S_{t}=S_{t+\Delta t}-S_{t}$ in small time interval from present time $t$ to a near future time $t+\Delta t$, where the return interval on the $[t, \Delta t \geq 0], \Delta S_{t} / S_{t}$.

Economically, we can divide this return into a systematic and random parts. In the systematic or deterministic part of stock return, we assume that the expected return over a period of the time is proportional to the length of that period. Accordingly, 
for a $\mu \in \mathbb{R}$ the expected increasing of the price, $\Delta S_{t}$, can be peresented by $\mu S_{t} \Delta t$. To model the random part of the stock return, we assume the price fluctuates stochastically where the variance of the returns is proportional of the interval of the time. Modeled by $\sigma \Delta B_{t}$ where $\Delta B_{t}$ represents the noise term (with variance $\Delta t$ ) driving the stock-price dynamics, and $\sigma>0$ is the parameter that describes the stock price fluctuation or the effect of noise. Empirically it has been established that the variance of the return equals to $\sigma^{2} \Delta t$ where $\sigma$ expresses the volatility of the stock. Combining these two parts of stock return, we will have

$$
\Delta S_{t}=S_{t}\left(\mu \Delta t+\sigma \Delta B_{t}\right), \quad S_{0}>0
$$

informally, by taking $\Delta t \rightarrow 0$, we have following stochastic differential equation:

$$
d S_{t}=S_{t}\left(\mu d t+\sigma d B_{t}\right), \quad S_{0}>0 .
$$

Equation (2.1) has the unique solution from Ito's formula and called Geometric Brownian motion

$$
S_{t}=S_{0} \exp \left(\left(\mu-\frac{1}{2} \sigma^{2}\right) t+\sigma B_{t}\right)
$$

Note that for any $t>0$ the log-returns

$$
\log \left(\frac{S_{t}}{S_{0}}\right)=\left(\mu-\frac{1}{2} \sigma^{2}\right) t+\sigma B_{t}
$$

have a $\mathrm{N}\left(\left(\mu-\frac{1}{2} \sigma^{2}\right) t, \sigma^{2} t\right)$ distribution. Equation (2.3) has the lognormal distribution which it entails form the basis for the Black-Scholes model of stock-price dynamics in continuous time. 


\subsubsection{The Black-Scholes Model Solution}

We show how the Black-Scholes model for valuing European call and put options on a stock works to trade continuously up to some fixed finite planning horizon .(Shoutens, 2003)

We assume two assets. One without risk like bank account and one risky like stock.

For no risk scenario, the price is given by $W=\left\{W_{t}=\exp (r t), 0 \leq t \leq T\right\}$.

For the risky scenario the price is modeled by

$$
S_{t}=S_{0} \exp \left(\left(\mu-\frac{1}{2} \sigma^{2}\right) t+\sigma B_{t}\right)
$$

where $B=\left\{B_{t}, t \geq 0\right\}$ is the Geometric Brownian motion, $\mu$ is drift and $\sigma$ is standard constant volatility over the time. By assuming the natural filtration $\mathbb{F}=$ $\left\{\mathbb{F}_{t}, t \geq 0\right\}$ generated by $B$, the stock price process $S=\left\{S_{t}, 0 \leq t \leq T\right\}$ is a strictly positive adapted process. We call this market model the Black-Scholes model.

The Black-Scholes formula for the European call and Put option is as

$$
\begin{gathered}
C:=C(K, T)=S_{0} N\left(d_{1}\right)-K \exp -r(T-t) N\left(d_{2}\right) \\
P:=P(K, T)=K \exp (-r T) N\left(-d_{2}\right)-S N\left(-d_{1}\right)
\end{gathered}
$$

where

$$
\begin{gathered}
d_{1}=\frac{\log \left(S_{0} / K\right)+\left(r+\frac{1}{2} \sigma^{2}\right)(T-t)}{\sigma \sqrt{T-t}} \\
d_{2}=d_{1}-\sigma \sqrt{T-t}
\end{gathered}
$$

$S_{0}:$ Stock price.

K: Strike price. 
r: Interest rate.

$\sigma$ : Volatility of underlying.

t: Current date.

T: Maturity date.

$T-t$ : Time to maturity.

\subsection{Levy Processes}

Looking at the definition of Brownian motion, we would like to have more general distribution than the Normal with independent and stationary increments process.However, in order to define such a stochastic process with independent and stationary increments, the distribution has to be infinitely divisible, such processes are called Levy processes, in honor of Paul Levy, the pioneer of the theory.

Definition 2.3. Let $\left(X_{t}\right)_{t \geq 0}$ be a stochastic process on the stochastic basis $\left(\Omega, \mathbb{F},\left(\mathbb{F}_{t}\right), P\right)$, $\left(X_{t}\right)$ is a real-valued random variable on $\Omega$.

The process $X=\left(X_{t}\right)_{t \geq 0}$ is said to be a Levy process if and only if:

(1) It has independent increments:

i.e. for any $n \in \mathbb{N}, \quad 0 \leq t_{1}<t_{2}<\ldots<t_{n}<\infty$

$$
X_{t_{k}}-X_{t_{k-1}} \quad(k=1,2, \ldots, n)
$$

are independent random variables.

(2) It has stationary increments:

i.e. for any $s<t, \quad X_{t}-X_{s}$ is equal in distribution to $X_{t-s}$. 
(3) It is Continuous

i.e. for $s \geq 0, \quad X_{t+s}-X_{s} \rightarrow 0 \quad$ as $t \rightarrow 0$ almost surely.

Brownian motion and the Poisson process are two well known examples of Levy processes. Inverse Gamma and Time-Changed processes are two other examples of Levy process and will be discussed in this chapter later (Schoutens,2003). Each process has a characteristic function which determines the distribution of the process. The characteristic function is always uniformly continuous and vailable.It is defined below. (Papapantoleon,2000)

Definition 2.4. Defined on a stochastic basis, for a real-valued process $\left(X_{t}\right)_{t \geq 0}$, $\phi_{X_{t}}(\theta)$ is the characteristic function of the process. If equals to $X_{t}$ :

$$
\phi_{X_{t}}(u)=E\left(e^{i u X_{t}}\right), \quad u \in \mathbb{R}
$$

The characteristic exponent of a Levy process is defined as (Sandri,1996)

$$
\Psi_{X_{t}}(u)=\frac{1}{t} \log \phi_{X_{t}}(u)
$$

The Levy-Khintchine Formula gives the characteristic function, which characterizes the distribution of a Levy process.

Theorem 2.5. Levy-Khinchine Formula

If $X=\left(X_{t}\right)_{t \geq 0}$ is a Levy process, then its characteristic function $\phi_{X_{t}}(u)$ is given by

$$
\phi_{X_{t}}(u)=\exp \left(\left[\left(i \mu u-\frac{1}{2} \sigma^{2} u^{2}+\int_{\mathbb{R}-0}\left(e^{i u x}-1-i u x \boldsymbol{I}_{|x|<1}\right) \Pi(d x)\right) t\right]\right)
$$

where $\mu \in \mathbb{R}, \sigma \geq 0, \boldsymbol{I}$ is the indicator function and $\Pi$ is the measure the jumps of $X$ and satisfying $\int_{\mathbb{R} \backslash\{0\}}$ inf $\left\{1, x^{2}\right\} \Pi(d x)<\infty$. 
A Levy process has three independent components: a linear drift, a Brownian motion and a compound Poisson process. The measure $\Pi(d x)$ represents the intensity and the length of the jumps. These three components,as well as LevyKhintchine representation, are fully determined by the Levy triplet $\left(\mu, \sigma^{2}, \Pi\right)$.A Brownian motion with drift is the only non-deterministic continuous Levy process.

$$
X_{t}=\mu t+\sigma B_{t}+Z_{t}
$$

where $Z_{t}$ is a Poisson process. The Levy-Ito Decomposition states that any Levy process $\left(X_{t}\right)_{t \geq 0}$ can be constructed as a sum of three independent processes.

\subsubsection{Examples of Levy Processes}

\section{Example 1. Poisson Process}

Let $\left(X_{t}\right)_{t \geq 0}$ be a random process with finite independent stationary increments. The Poisson analysis counts how many jumps in increments occur during the interval $[0, t]$. See (Rama\&Tankov).The increments are independent of the past, for example, if there is an average of five jumps within an interval, not observing a jump would not change the probability of happening a jump in the future.

Definition 2.6. $\left(N_{t}\right)_{t \geq 0}$ is a Poisson process if and only if:

(1) $N_{0}=0$

(2) $\left(N_{t}\right)_{t \geq 0}$ has stationary and independent increments.

(3) $P\left(N_{t}=n\right)=e^{-\lambda t \frac{(\lambda t)^{n}}{n !}}, \quad n=0,1,2, \ldots$

See (Boxma, Yechiali) for a proof. 


\section{Example 2. Brownian Motion}

Modeling the price process using geometric Brownian motion leads to calculating the expected value dependent on the spot price. Brownian Motion is described by the Wiener process, which is defined below.

The probability density function of $\left(B_{t}\right)$ is defined by:

$$
f_{B_{t}}(x)=\frac{1}{\sqrt{2 \pi t}} e^{-\frac{x^{2}}{2 t}}
$$

\subsection{Time-Changed Subordinator}

Definition 2.7. A Subordinator $A_{t}$ is a nonnegative and nondecreasing Levy process.

Definition 2.8. Let $\left(A_{t}\right)_{t \geq 0}$ be a subordinator and $\left(B_{t}\right)$ is a Brownian motion then $\left(B_{A_{t}}\right)_{t \geq 0}$ is Time-Changed process.

The characteristic fuction $\phi_{B_{A_{t}}}$ of Time-Changed model is given by below

$$
\phi_{B_{A_{t}}}(u)=E\left(e^{i u B_{A_{t}}}\right), \quad u \in \mathbb{R}
$$

We have more details in chapter three regarding to Time-Changed model.

\subsubsection{Inverse Gaussian Subordinator}

The Inverse Gaussian distribution is a two parameter $(a, b)$ family of continuous probability distributions with support on $(0, \infty)$.

Its probability density function is given by

$$
f(x ; a, b)=\left(\frac{b}{2 \pi x^{3}}\right)^{\frac{1}{2}} \exp \frac{-b(x-a)^{2}}{2 a^{2} x}
$$


for $x>0$, where $a>0$ is the mean and $b>0$ is the shape parameter.

As $b \rightarrow \infty$ this distribution tends to a normal distribution.

To indicate that a random variable $\mathrm{X}$ is Inverse Gaussian distributed with mean a and shape parameter b we write $X \sim I G(a, b)$.

Definition 2.9. $\left(A_{t}\right)$ is an IG-Subordinator if

$$
A_{t+s}-A_{s} \sim I G(a t, b) \quad s, t \geq 0 .
$$

An IG-subordinator has characteristic exponent:

$$
\Psi_{A_{(t)}}^{I G}(u)=-a t\left(\sqrt{-2 i u+b^{2}}-b\right) \quad a, b>0
$$




\section{Chapter 3}

\section{BARRIER PRICING BASED ON CONVENTIONAL BLACK-SCHOLES MODEL}

Since introduced it in the market, barrier option have become the most popular class of exotic options. They have been traded on stocks, oil, gas, currency and the other commodities.

In this chapter, we provide the details on barrier option pricing based on BlackScholes model. Both univariate and bivariate models for single asset and two assets pricing formulas are introduced with focus on Brent oil, WTI oil and Ontario gasoline as univariate assets and oil/gasoline as bivariate assets. The objective is to use the already existing oil and gasoline data to predicts the option price for future by using Black-Scholes model. Of course, the models will be offer from the same short coming of Black-Scholes model for vanilla option. Which will be presented in the next chapters with compared with advance Time-Changed models.

\subsection{Barrier Option for Black-Scholes Model}

Oil market's barrier options have been traded in the OTC since 1967. The Chicago Board Option Exchange and the American Option Exchange now list up-and-out 
barrier call (UOBC)options and down-and-out put (DOBP) options on stock indexes.(Hauge)

\subsubsection{Univariate Black-Scholes Model}

Merton (1973) and Reiner and Rubinstein (1991) have developed formulas for pricing standard barrier options using Black-Scholes model. Their formulas are different but they use a common set of factors.

We started with Down-and-In-barrier call option (DIBC) where we can have two scenarios. First when the barrier price $(\mathrm{M})$ is smaller than the strike price $(\mathrm{K})$ and the second scenario is when the barrier price is larger than the strike price.

To be able to extract the option price for these two scenarios based on Black-Scholes model we need to define the density of the risk neutral return of a asset as normal distribution as follow

$$
f(u) \equiv\left(\frac{1}{\sigma \sqrt{2 \pi t}}\right) e^{-\frac{1}{2 v^{2}}}
$$

with

$$
\begin{gathered}
v \equiv \frac{(u-\mu t)}{\sigma \sqrt{t}} \\
\mu \equiv \log \left(\frac{r}{d}\right)-\frac{1}{2 \sigma^{2}}
\end{gathered}
$$

This the normal density where $\mathrm{r}$ is the interest, $\mathrm{d}$ is the payout of the underlying asset, sigma is the volatility of the underlying asset, and t is the time to expiration. We will need another density where the price passes the barrier.

$$
g(u) \equiv e^{\frac{2 \eta \alpha}{\sigma^{2}}}\left(\frac{1}{\sigma \sqrt{2 \pi t}}\right) e^{-\frac{1}{2 v^{2}}}
$$


with

$$
\begin{gathered}
v \equiv \frac{(u-2 \eta \alpha-\eta \mu t)}{\sigma \sqrt{t}} \\
\alpha \equiv \log \left(\frac{M}{S_{0}}\right)
\end{gathered}
$$

Accordingly for DIBC and scenario one when $K>M$ we may have

(1) $S_{T}>K$ providing $S(t) \geq M$ for some $0 \leq t<T$ (Figure 3.1 a)

In that case, pay - off $=S_{T}-K$.

(2) $S_{T} \leq K$ providing $S(t) \geq M$ for some $0 \leq t<T$ (Figure $3.1 \mathrm{~b}$ ). In that case, pay - off $=0$.

(3) There is a case where the price will never hit the barrier $S(t)>M$ for all $\mathrm{t}$ and the option will not be activated at all (Figure $3.1 \mathrm{c}$ ). In that case, the Pay - of $f=$ rebate. 
Figure 3.1: Differnet types of DIBC when $K>M$
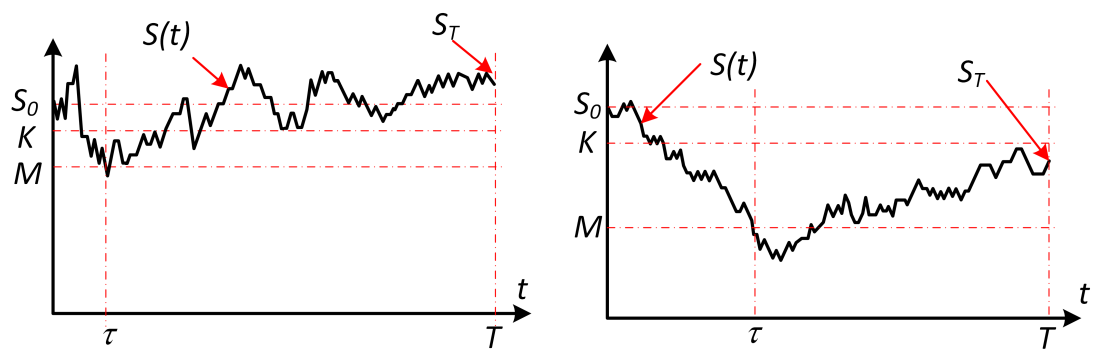
(a) $S_{T}>K, S(t) \geq M$ for some
(b) $S_{T} \geq K, S(t) \leq M$ for some
$0 \leq t<T$
$0 \leq t<T$

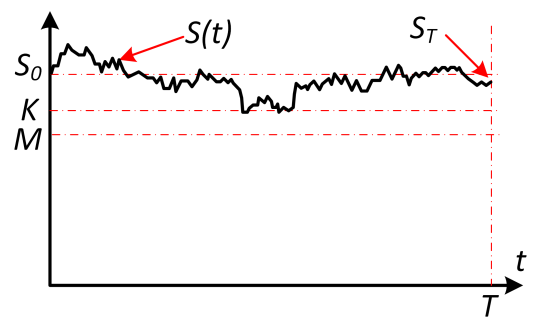

(c) $S(t)>M$ for all t 
For DIBC we can have another scenario where $K<M$.

(1) For this scenario, we can have a case where $S_{T}>M$ providing $S(t) \geq M$ for some $0 \leq t<T$ (Figure 3.2 a). In that case Pay-off $=S_{T^{-}} K$.

(2) We may have a case where $S(T) \geq M$ and $S(T)>K$ (Figure 3.2 b) which leads to Pay - off $=S_{T}-K$.

(3) For the same scenario we may have $S(T) \geq K$ as depicted in Figure (3.3 c). In that case Pay - off $=0$.

(4) For the final case we have a case where $\mathrm{S}(\mathrm{t})$ never pass the barrier for all $\mathrm{t}$ as presented in Figure $(3.4 \mathrm{~d})$ which end up for Pay - off $=$ rebate. 
Figure 3.2: Differnet types of DIBC when $K<M$
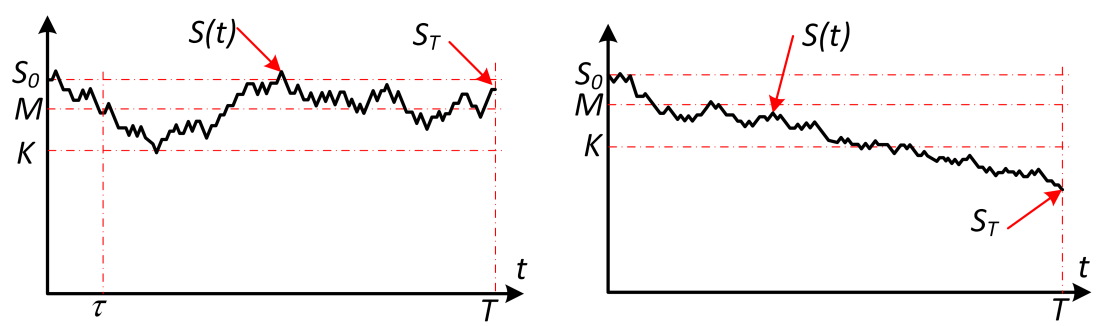

(a) $S(T)>M$ for all t

(b) $S(T)>K S(T) \geq M$ for all t
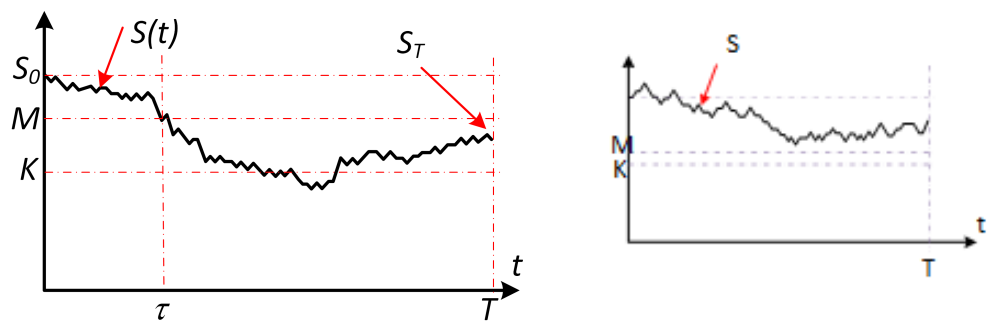

(c) $S(T) \geq K$ for all t

(d) $K<M$ for all t 


\section{Consequently,}

$$
\begin{gathered}
A \equiv r^{-T} \int \phi\left(S_{0} e^{u}-K\right) f(u) d u=\phi S_{0} N\left(\phi x_{1}\right)-\phi K e^{-r T} N\left(\phi x_{1}-\phi \sigma \sqrt{T}\right) \\
B \equiv r^{-T} \int \phi\left(S_{0} e^{u}-K\right) f(u) d u=\phi S_{0} N\left(\phi x_{2}\right)-\phi K e^{-r T} N\left(\phi x_{2}-\phi \sigma \sqrt{T}\right) \\
C \equiv r^{-T} \int \phi\left(S_{0} e^{u}-K\right) g(u) d u=\phi S_{0}\left(\frac{M}{S_{0}}\right)^{2(\mu+1)} N\left(\eta y_{1}\right)-\phi K e^{-r T}\left(\frac{M}{S_{0}}\right)^{2 \mu} N\left(\eta y_{1}-\eta \sigma \sqrt{T}\right) \\
D \equiv r^{-T} \int \phi\left(S_{0} e^{u}-K\right) g(u) d u=\phi S_{0}\left(\frac{M}{S_{0}}\right)^{2(\mu+1)} N\left(\eta y_{2}\right)-\phi K e^{-r T}\left(\frac{M}{S_{0}}\right)^{2 \mu} N\left(\eta y_{2}-\eta \sigma \sqrt{T}\right) \\
E \equiv R r^{-T} \int(f(u)-g(u)) d(u) d u=R e^{-r T}\left[N\left(\eta x_{2}-\eta \sigma \sqrt{T}\right)-\left(\frac{M}{S_{0}}\right)^{2 \mu} N\left(\eta y_{2}-\eta \sigma \sqrt{T}\right)\right] \\
F \equiv=R\left[\left(\frac{M}{S_{0}}\right)^{(\mu+\lambda)} N(\eta z)+\left(\frac{M}{S_{0}}\right)^{(\mu-\lambda)} N(\eta z-2 \eta \lambda \sigma \sqrt{T})\right]
\end{gathered}
$$

Where

$$
\begin{gathered}
x_{1}=\frac{\ln \left(\frac{S_{0}}{K}\right)}{\sigma \sqrt{T}}+(1+\mu) \sigma \sqrt{T} ; \\
x_{2}=\frac{\ln \left(\frac{S_{0}}{M}\right)}{\sigma \sqrt{T}}+(1+\mu) \sigma \sqrt{T} ; \\
y_{1}=\frac{\ln \left(\frac{M^{2}}{S_{0} K}\right)}{\sigma \sqrt{T}}+(1+\mu) \sigma \sqrt{T} ; \\
y_{2}=\frac{\ln \left(\frac{M}{S_{0}}\right)}{\sigma \sqrt{T}}+(1+\mu) \sigma \sqrt{T} ; \\
z=\frac{\ln \left(\frac{M}{S_{0}}\right)}{\sigma \sqrt{T}}+\lambda \sigma \sqrt{T} ; \\
\mu=\frac{r-\frac{\sigma^{2}}{2}}{\sigma^{2}} ; \\
\lambda=\sqrt{\mu^{2}+\frac{2 r}{\sigma^{2}}} ; \\
\eta \begin{array}{c}
\text { if } \text { Down } \\
-1 \quad \text { if } U p
\end{array}
\end{gathered}
$$




$$
\phi= \begin{cases}1 & \text { if Call } \\ -1 & \text { if Put }\end{cases}
$$

The Price for all barrier option cases is provided in following table (3.1) and (3.2).

\section{"In" Barrier Options}

As explained before, In options are paid for today but first come into existence if the asset price $S_{0}$ hits the barrier M before maturity time.

\begin{tabular}{|c|c|c|c|}
\hline Type & & $K<M$ & $K>M$ \\
\hline \hline Down-and-in Call & $S_{0}>M$ & $A-B+D+E$ & $C+E$ \\
\hline Up-and-in Call & $S_{0}<M$ & $B-C+D+E$ & $A+E$ \\
\hline Down-and-in Put & $S_{0}>M$ & $A+E$ & $B-C+D+E$ \\
\hline Up-and-in Put & $S_{0}<M$ & $C+E$ & $A-B+D+E$ \\
\hline
\end{tabular}

Table 3.1: Prices of In Univariate Barrier Option

\section{"Out" Barrier Options}

As explained before, Out options are same as the standard options except that the option becomes worthless if the asset price $S_{0}$ hits the barrier M before T. 


\begin{tabular}{|c|c|c|c|}
\hline Type & & $K<M$ & $K>M$ \\
\hline \hline Down-and-out Call & $S_{0}>M$ & $B-D+F$ & $A-C+F$ \\
\hline Up-and-out Call & $S_{0}<M$ & $A-B+C-D+F$ & $F$ \\
\hline Down-and-out Put & $S_{0}>M$ & $F$ & $A-B+C-D+F$ \\
\hline Up-and-out Put & $S_{0}<M$ & $A-C+F$ & $B-D+F$ \\
\hline
\end{tabular}

Table 3.2: Prices of Out Univariate Barrier Option.

\subsubsection{Empirical Data}

The empirical data is composed of historic daily prices for the commodities, Brent oil, WTI oil, and weekly average price for gasoline in Ontario, Canada, obtained from for a five year period. As presented table (1.2) and calculated from the logreturn empirical pricing data of each commodities.

Since we need $\operatorname{drift}(\mu)$ and volatility $(\sigma)$ for each commodities in MATLAB, and by (2.3) log- return price in Black-Scholes model have a normal distribution $N((\mu-$ $\left.\left.\frac{1}{2} \sigma^{2}\right) t, \sigma^{2} t\right)$, then we can find the drift and volatility for any asset by using

$$
\begin{gathered}
\text { Mean }=\left(\mu-\frac{1}{2} \sigma^{2}\right) t, \\
\text { Variance }=\sigma^{2} t
\end{gathered}
$$

where the $t=1$ for Brent and WTI oil, because we have daily price and for Ontario 
gasoline weekly price is 5 .

\begin{tabular}{ccccc}
\hline Series & $\mu_{\text {daily }}$ & $\sigma_{\text {daily }}$ & $\mu_{\text {weekly }}$ & $\sigma_{\text {weekly }}$ \\
\hline \hline BRE Oil & 0 & 0.02 & 0.006 & 0.002 \\
WTI Oill & 0 & 0.02 & 0.005 & 0.013 \\
Ontario Gasoline & - & - & 0.031 & 0.014
\end{tabular}

Table 3.3: $\mu$ and $\sigma$ for all three assets

\subsection{Pricing Under Univariate Black-Scholes Model}

To describe the dynamics of the prices for the barrier option contracts under BlackScholes model, three dimensional graphs have been created. Figures (3.3), (3.4) and (3.5) describe the price for Down-and-Out call barrier option for Brent, WTI oil and Ontario gasoline versus the maturity time $(\mathrm{T})$ and strike price $(\mathrm{K})$. As we expected based on payoff formula, the option price decreases, when the strike price increases. As the maturity increases, there is a slight decrease in the option prices. The maturity time range taken for these graphs was $0.08,0.16,0.25,0.5,0.6,0.75$ and 1 year.

Figures (3.6), (3.7) and (3.8) demonstrate interaction between Down-and-Out call barrier option for our three assets, strike price $(\mathrm{K})$ and the volatility of assets $(\sigma)$. In all graphs, the option price has a highest price in the lowest $\mathrm{K}$ and $\sigma$ and the price slight decrease when $\mathrm{K}$ and $\sigma$ increasing. 
Figure 3.3: Simulation of option price for Brent Oil in Black-Scholes Model

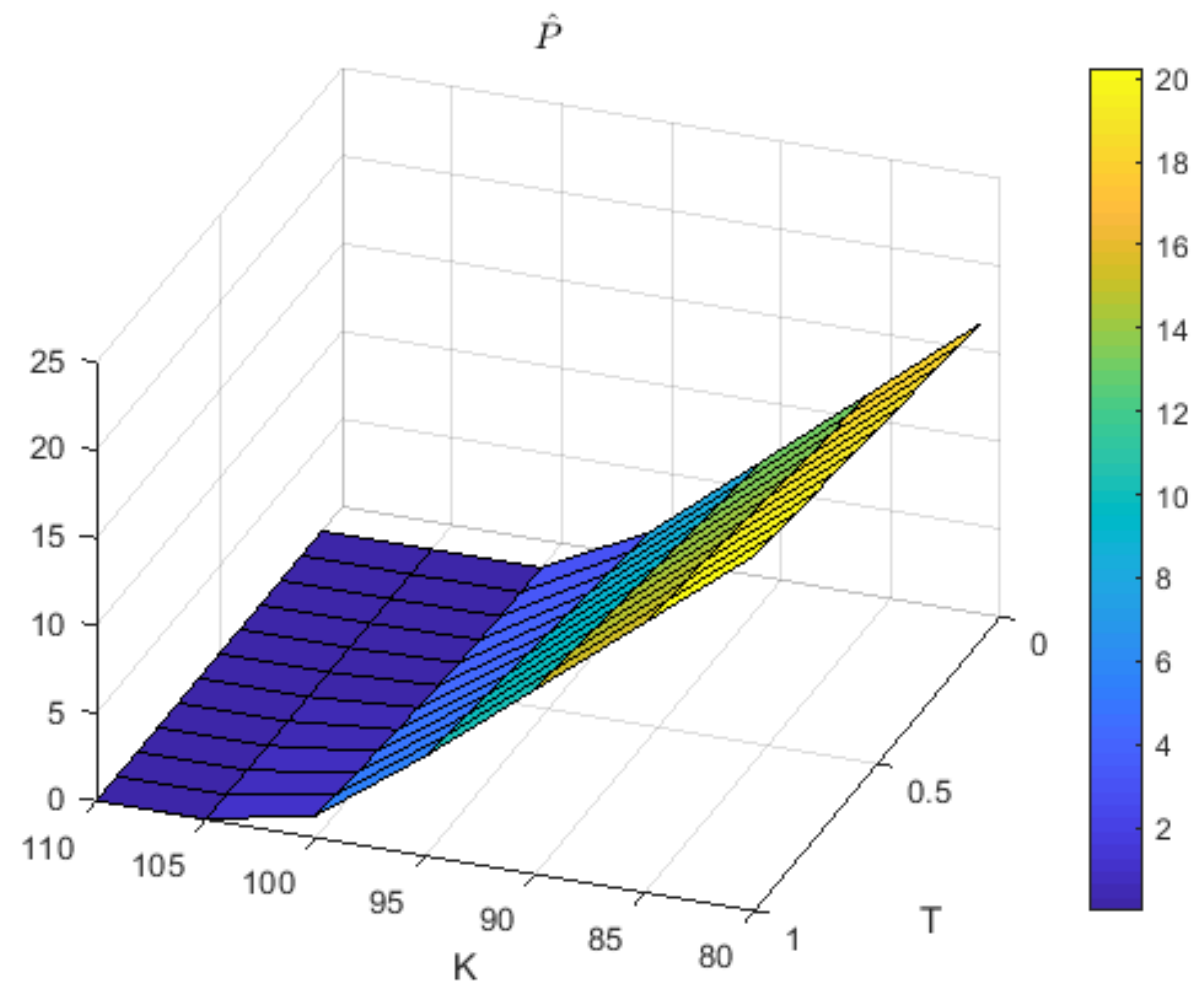

Prices for Brent oil: The 3-D graph shows the barrier option prices change versus $\mathrm{T}$ ( maturity of the barrier option) per year, $\mathrm{K}$ (Strike price of the barrier option) based on univariate Black-Scholes model simulation. The $\mathrm{K}$ is [80:5:110] and $\mathrm{T}$ is $\left[\frac{1}{12}: \frac{1}{12}: 1\right]$ 
Figure 3.4: Simulation of option price for WTI Oil in Black-Scholes Model

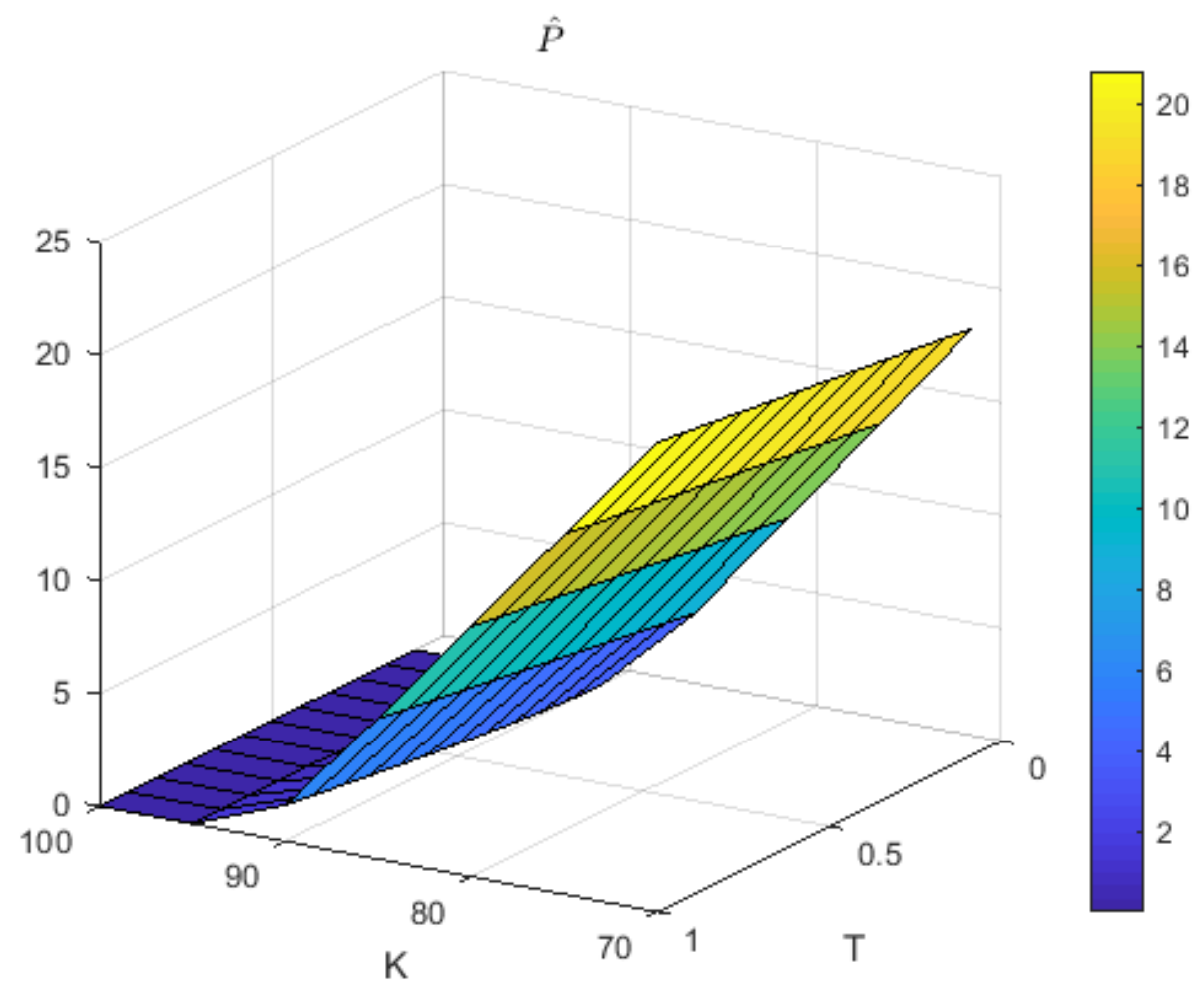

Prices for WTI oil: The 3-D graph shows the interaction between $\mathrm{T}$ ( maturity of the barrier option), $\mathrm{K}$ (Strike price of the barrier option) and the price of the option using univariate Black-Scholes model simulation. The rang for $\mathrm{K}$ is $70,75, \ldots, 100$. 
Figure 3.5: Simulation of option price for Ontario Gasoline in Black-Scholes Model

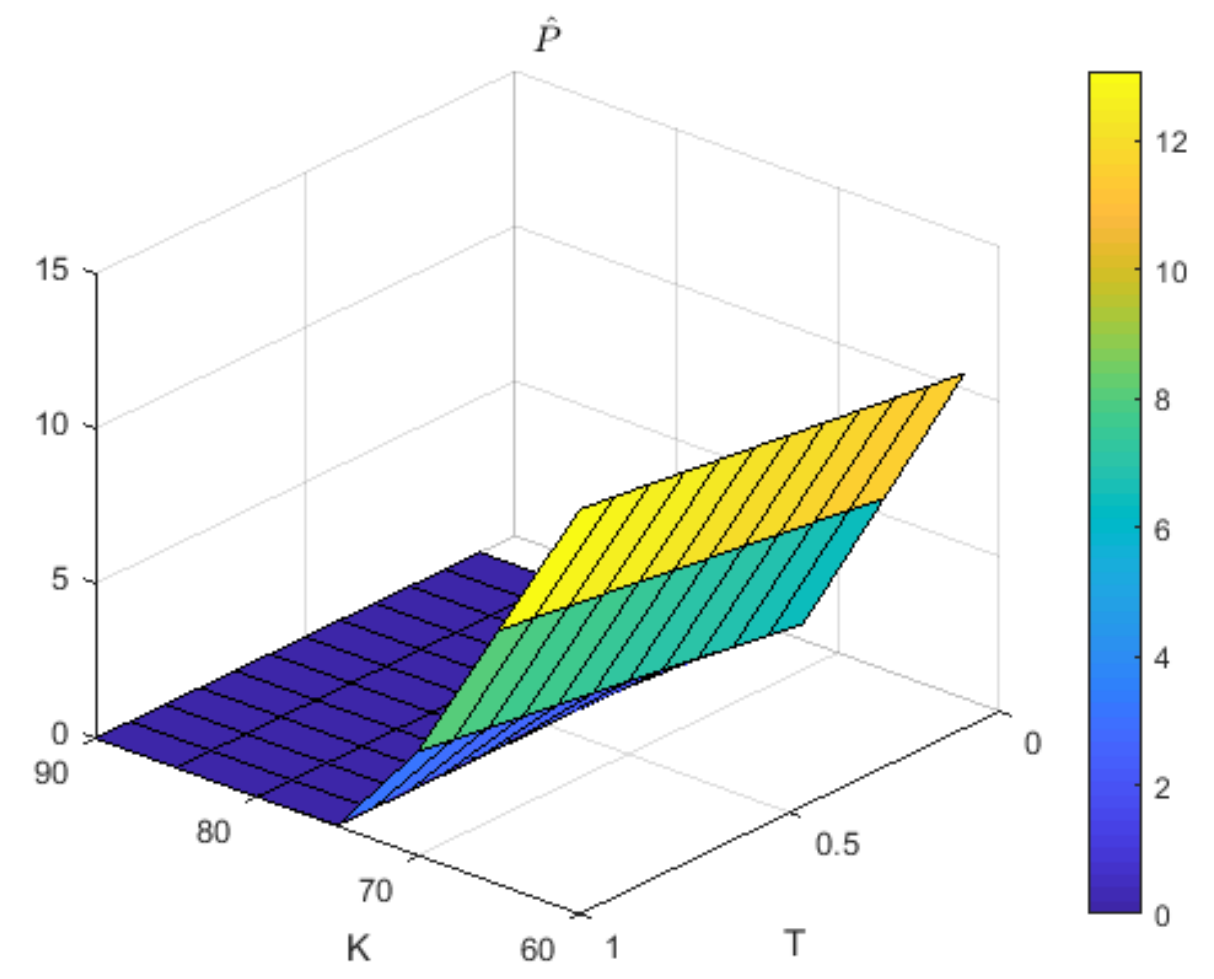

Prices for Ontario gasoline: The 3-D graph shows the interaction between $\mathrm{T}($ maturity of the barrier option), $\mathrm{K}$ (Strike price of the barrier option) and the price of the option using univariate Black-Scholes model simulation. The range for $\mathrm{K}$ is $60,65, \ldots, 90$ 
Figure 3.6: Simulation of option price for Brent Oil in Black-Scholes Model

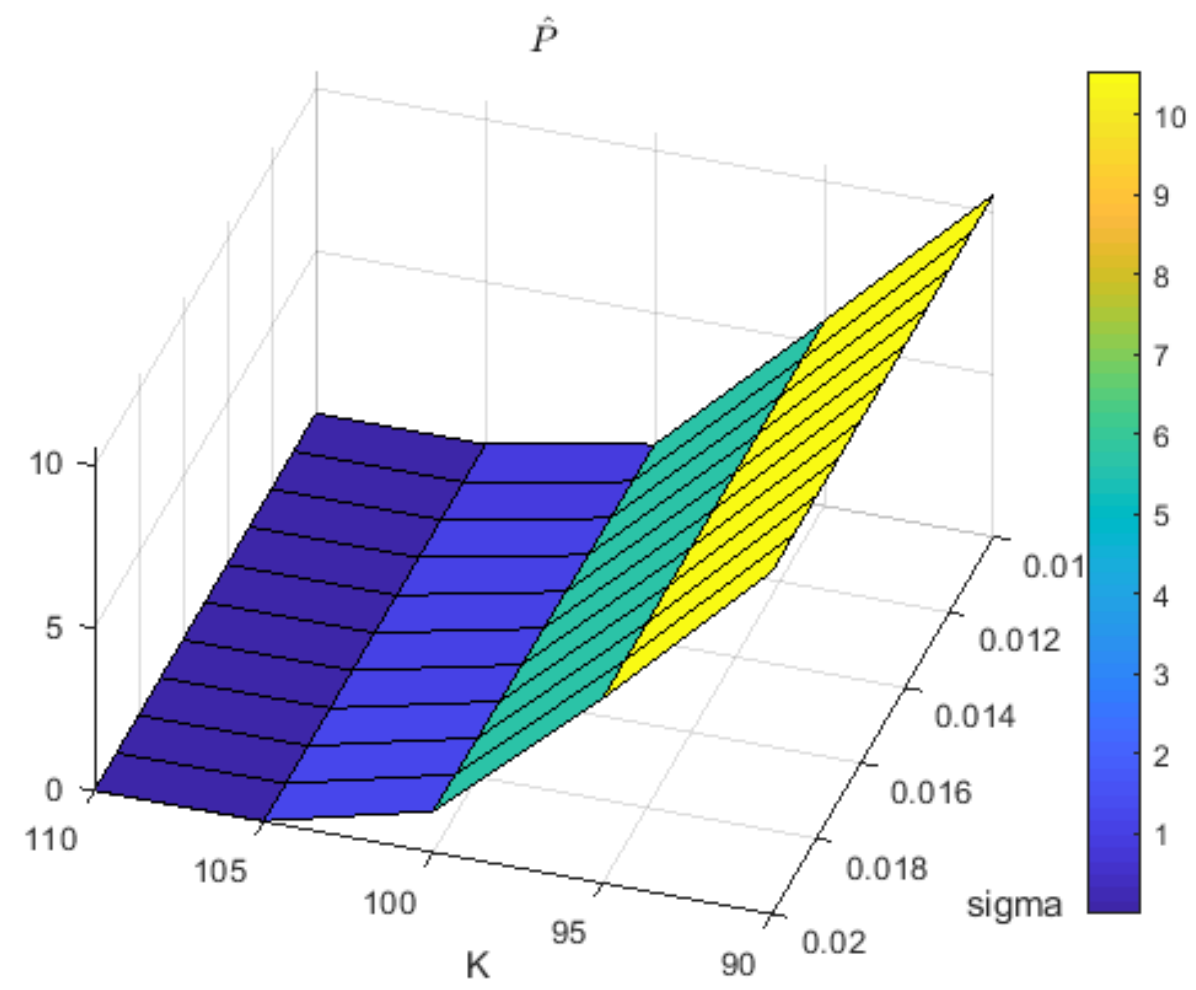

Prices for Brent oil: The 3-D graph shows the interaction between $\sigma_{\text {BrentOil }}$,

$\mathrm{K}$ (Strike price of the barrier option) and the price of the option using univariate

Black-Scholes model simulation. $\sigma_{\text {BrentOil }}$ is [0.01:0.001:0.02]. 
Figure 3.7: Simulation of option price for WTI Oil in Black-Scholes Model

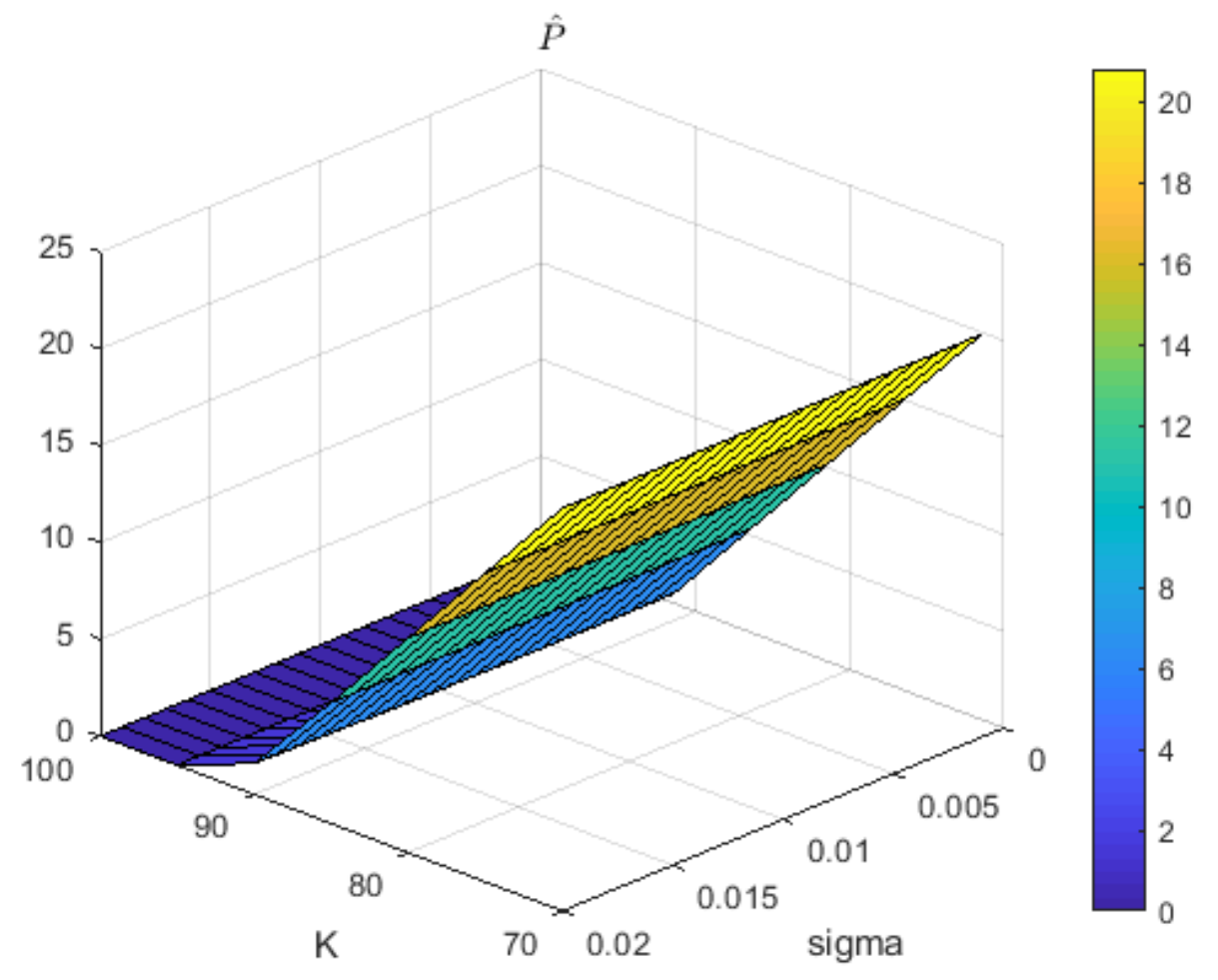

Prices for WTI oil: The 3-D graph shows the interaction between $\sigma_{W T I O i l}$, $\mathrm{K}$ (Strike price of the barrier option) and the price of the option using univariate Black-Scholes model simulation. $\sigma_{W T I O i l}$ is [0.01:0.001:0.02]. 
Figure 3.8: Simulation of option price for Ontario Gasoline in Black-Scholes Model

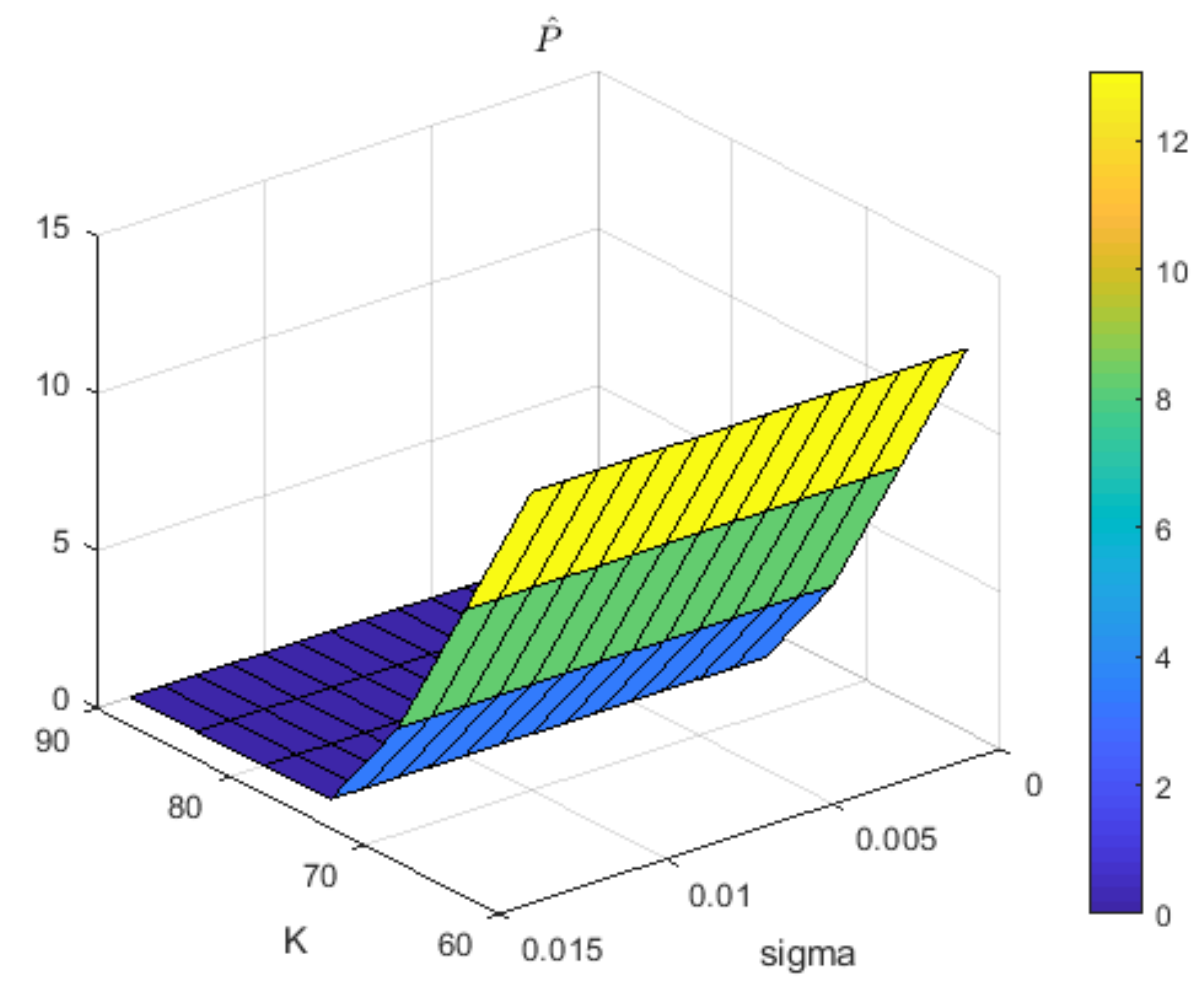

Prices for Ontario gasoline: The 3-D graph shows the interaction between

$\sigma_{\text {OntarioGasoline }}, \mathrm{K}$ (Strike price of the barrier option) and the price of the option using uni-variate Black-Scholes model simulation. $\sigma_{\text {OntarioGasoline }}$ is

$$
\text { [0.01:0.001:0.014] }
$$




\subsubsection{Bivariate Black-Scholes Model}

In this section, we explain the payoff formula for barrier option for two assets, that assets are based on Black-Scholes model and they follow geometric Brownian motions

$$
\begin{aligned}
& d S_{t}^{1}=S_{t}^{1}\left(\mu_{1} d t+\sigma_{1} d z_{t}\right) \\
& d S_{t}^{2}=S_{t}^{2}\left(\mu_{2} d t+\sigma_{2} d w_{t}\right)
\end{aligned}
$$

where $\mu_{1}$ and $\mu_{2}$ are the expected increasing rate of return of the two assets, and $\sigma_{1}$ and $\sigma_{2}$ are the volatility of assets. $d z$ and $d w$ are two Brownian Processes.

In a two-asset barrier option, one of the underlying assets, $S_{1}$, determines how much the option is in- or out-of-the-money, and the other asset, $S_{2}$, is linked to barrier hits. Heynen and Kat (1994) have developed the following pricing formula(Hauge):

$$
\begin{aligned}
P & =\eta S_{0}^{1}\left\{M\left(\eta d_{1}, \phi e_{1} ;-\eta \phi \rho\right)\right. \\
& \left.-\exp \left[\frac{2\left(\mu_{2}+\rho \sigma_{1} \sigma_{2}\right) \ln \left(\frac{M}{S_{0}^{2}}\right)}{\sigma_{2}^{2}}\right] M\left(\eta d_{3}, \phi e_{3} ;-\eta \phi \rho\right)\right\} \\
& -\eta K e^{-r T}\left\{M\left(\eta d_{2}, \phi e_{2} ;-\eta \phi \rho\right)\right. \\
& \left.-\exp \left[\frac{2 \mu_{2} \ln \frac{M}{S_{0}^{2}}}{\sigma_{2}^{2}}\right] M\left(\eta d_{4}, \phi e_{4} ;-\eta \phi \rho\right)\right\}
\end{aligned}
$$

Where

$$
\begin{gathered}
d_{1}=\frac{\ln \left(\frac{S_{0}^{1}}{K}\right)+\left(\mu_{1}+\sigma_{1}^{2}\right) T}{\sigma_{1} \sqrt{T}} \\
d_{2}=d_{1}-\sigma_{1} \sqrt{T} \\
d_{3}=d_{1}+\frac{2 \rho \ln \left(\frac{M}{S_{0}^{2}}\right)}{\sigma_{2} \sqrt{T}}
\end{gathered}
$$




$$
\begin{gathered}
d_{4}=d_{2}+\frac{2 \rho \ln \left(\frac{M}{S_{0}^{2}}\right)}{\sigma_{2} \sqrt{T}} \\
e_{1}=\frac{\ln \left(\frac{M}{S_{0}^{2}}\right)+\left(\mu_{2}+\rho \sigma_{1} \sigma_{2}\right) T}{\sigma_{2} \sqrt{T}} \\
e_{2}=e_{1}+\rho \sigma_{1} \sqrt{T} \\
e_{3}=e_{1}-\frac{2 \ln \left(\frac{M}{S_{2}}\right)}{\sigma_{2} \sqrt{T}} \\
d_{4}=d_{2}-\frac{2 \ln \left(\frac{M}{S_{2}}\right)}{\sigma_{2} \sqrt{T}} \\
\mu_{1}=-\sigma_{1}^{2} \\
\mu_{1}=-\sigma_{2}^{2}
\end{gathered}
$$

and $M(a, b ; \rho)$ is bivariate cumulative normal distribution function.

\section{Two-Asset Out Barriers Option}

Down-and-out call $\left(c_{d o}\right), \quad \eta=1, \phi=-1$

$$
P= \begin{cases}\max \left(S_{1}-K ; 0\right) & \text { if } S_{2}>M \\ 0 & \text { else }\end{cases}
$$

Up-and-out call $\left(C_{u o}\right), \quad \eta=1, \phi=1$

$$
P= \begin{cases}\max \left(S_{1}-K ; 0\right) & \text { if } S_{2}<M \\ 0 & \text { else }\end{cases}
$$

Down-and-out put $\left(p_{d o}\right), \quad \eta=-1, \phi=-1$

$$
P= \begin{cases}\max \left(K-S_{1} ; 0\right) & \text { if } S_{2}>M \\ 0 & \text { else }\end{cases}
$$


Up-and-out put $\left(p_{u o}\right), \quad \eta=-1, \phi=1$

$$
P= \begin{cases}\max \left(K-S_{1} ; 0\right) & \text { if } S_{2}<M \\ 0 & \text { else }\end{cases}
$$

Two-Asset In Barriers Option

Down-and-in call, $\quad c_{d i}=$ Call $-c_{d o}$

$$
P= \begin{cases}\max \left(S_{1}-K ; 0\right) & \text { if } S_{2}>M \\ 0 & \text { else }\end{cases}
$$

Up-and-in call, $\quad c_{u i}=$ call $-c_{u o}$

$$
P= \begin{cases}\max \left(S_{1}-K ; 0\right) & \text { if } S_{2}<M \\ 0 & \text { else }\end{cases}
$$

Down-and-in put,$\quad p_{d i}=$ put $-p_{d o}$

$$
P= \begin{cases}\max \left(K-S_{2} ; 0\right) & \text { if } S_{2}>M \\ 0 & \text { else }\end{cases}
$$

Up-and-in put, $\quad p_{u i}=p u t-p_{u o}$

$$
P= \begin{cases}\max \left(K-S_{2} ; 0\right) & \text { if } S_{2}<M \\ 0 & \text { else }\end{cases}
$$




\subsection{Pricing Under Bivariate Black-Scholes Model}

To describe the dynamics of prices for the barrier option contracts under bivariate Black-Scholes model, three dimensional graphs have been created. We apply bivariate Black-Scholes model for three sets: Brent oil and Ontario gasoline, WTI oil and Ontario gasoline, and Brent and WTI oil.

Figures (3.9), (3.10) and (3.11) demonstrate the price of bivariate Down-and-Out call barrier option versus the maturity time $(\mathrm{T})$ and strike price $(\mathrm{K})$. As we expected by increasing strike price for Brent oil for two first sets and increasing WTI strike price for last set the barrier option price decreases. Figures (3.12), (3.13) and (3.14) show that for bivariate barrier option changing volatilities of all assets does not change the price to much. Changing bivariate barrier option price versus the correlation between underlying assets and the strike price (same as strike price for first three graphs in this section) have been presented in figures (3.15), (3.16) and (3.17). Bivariate barrier option price decreases by increasing strike price and changing correlation $(\rho)$ does not have an effect on the option price. 
Figure 3.9: Barrier Option Prices for Two Assets(Brent Oil and Onatrio Gasoline)by using Bivariate Black-Scholes Model

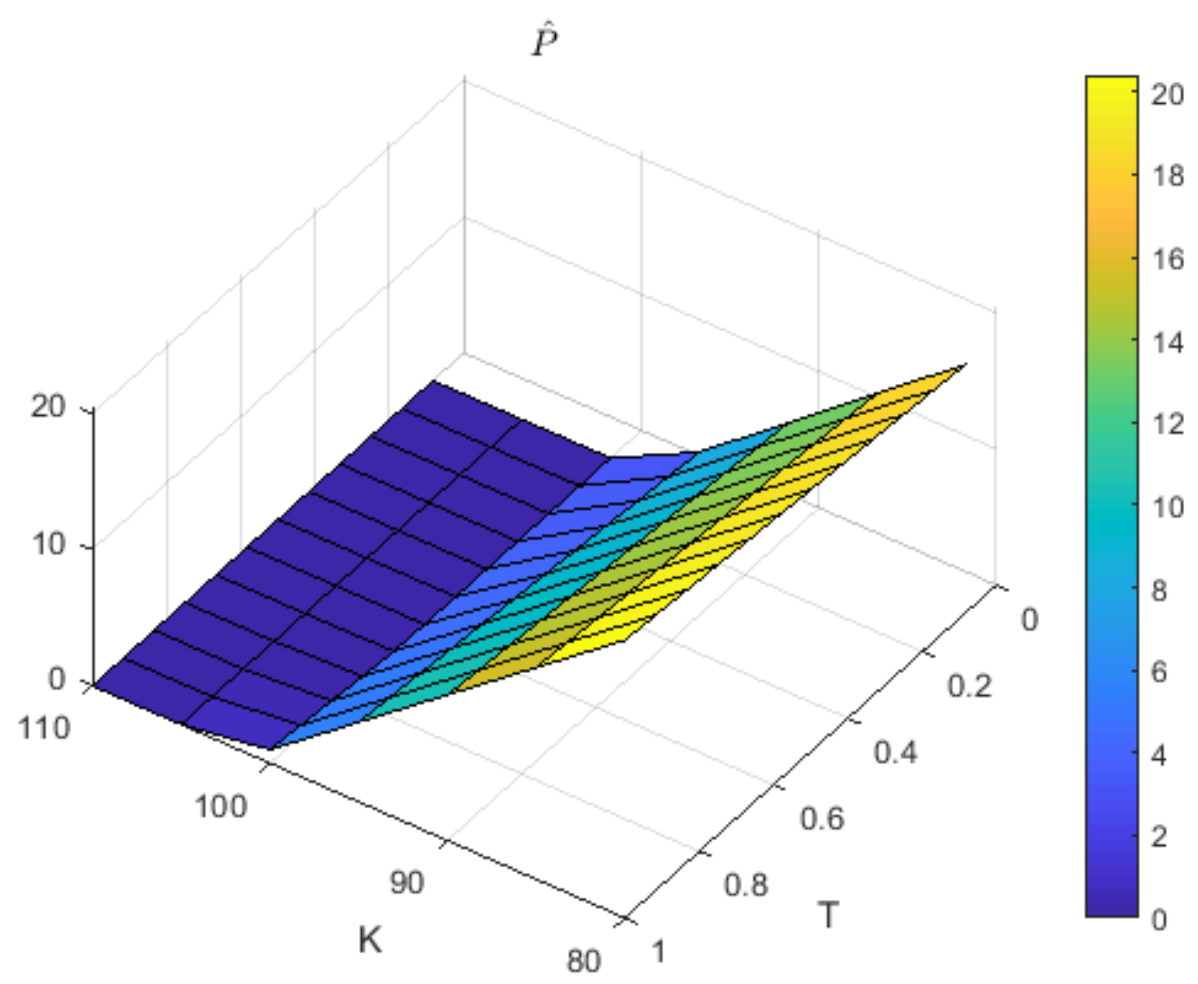

Barrier Prices Option: The 3-D graph shows the interaction between $\mathrm{T}$ ( maturity time of the barrier option), $\mathrm{K}$ (strike price of the barrier option) and the price of the option for two assets using bivariate Black-Scholes model simulation. The $\mathrm{K}$ is [80:5:110], T is $\left[\frac{1}{12}: \frac{1}{12}: 1\right]$ and and $\mathrm{M}$ is 68 . 
Figure 3.10: Barrier Option Prices for Two Assets(WTI Oil and Onatrio Gasoline)by using Bivariate Black-Scholes Model

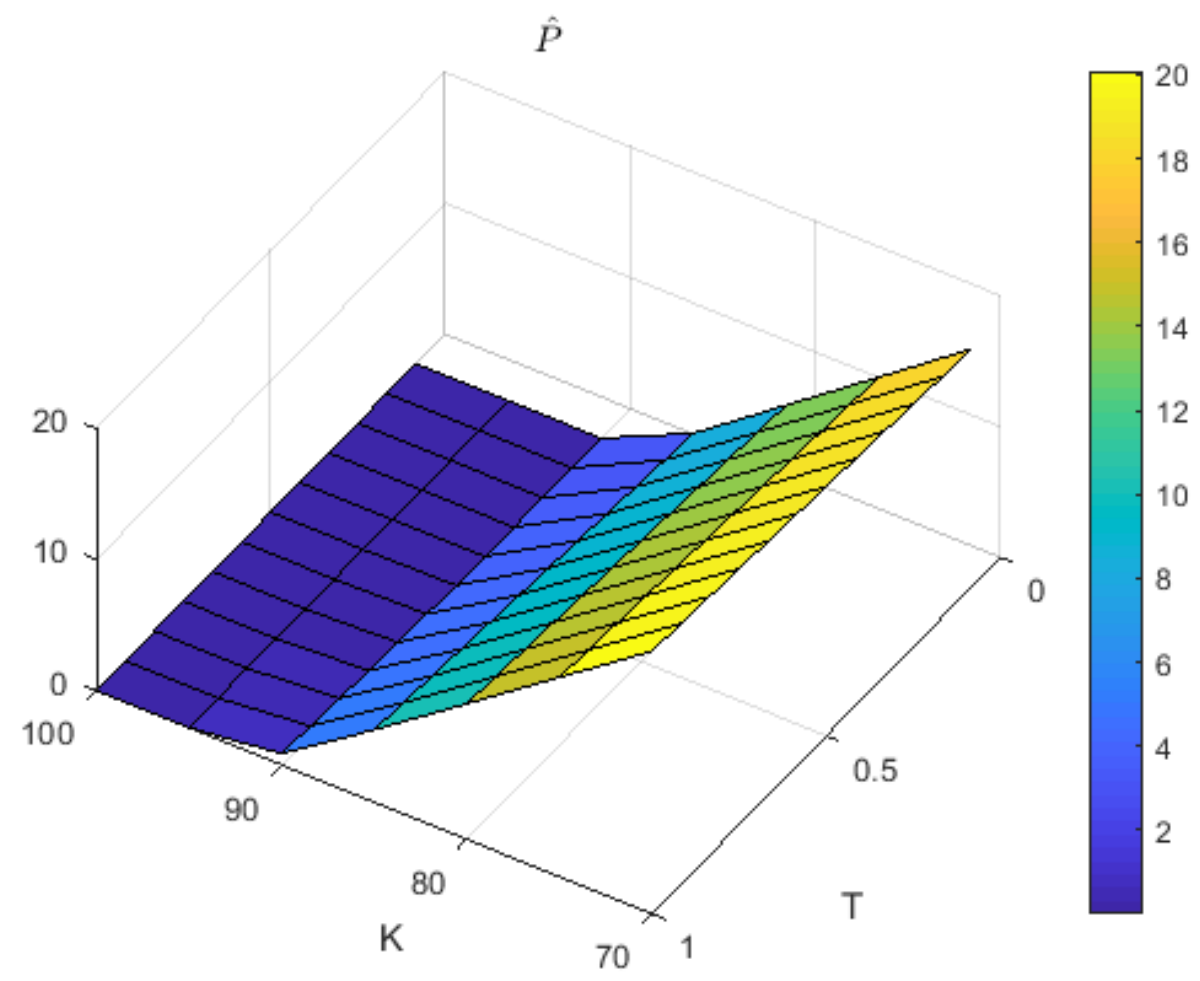

Barrier Prices Option: The 3-D graph shows the interaction between T( maturitytime of the barrier option), $\mathrm{K}$ (strike price of the barrier option) and the price of the option for two assets using bivariate Black-Scholes model simulation.

The $\mathrm{K}$ is $[70: 5: 100], \mathrm{T}$ is $\left[\frac{1}{12}: \frac{1}{12}: 1\right]$ and $\mathrm{M}$ is 68 . 
Figure 3.11: Barrier Option Prices for Two Assets(Brent and WTI oil)by using Bivariate Black-Scholes Model

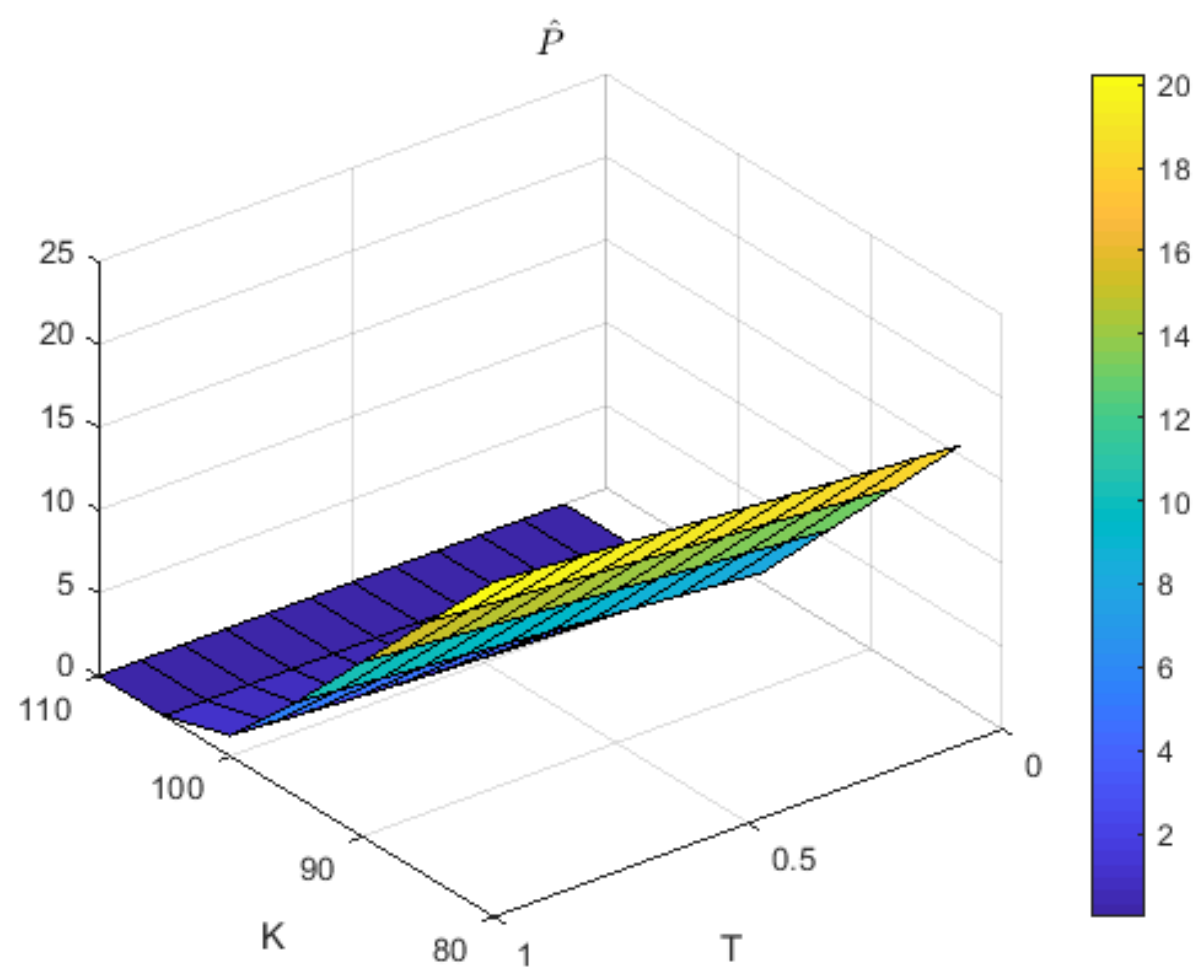

Barrier Prices Option: The 3-D graph shows the interaction between T( maturity time of the barrier option), $\mathrm{K}$ (strike price of the barrier option) and the price of the option for two assets using bivariate Black-Scholes model simulation. The $\mathrm{K}$ is $[80: 5: 110], \mathrm{T}$ is $\left[\frac{1}{12}: \frac{1}{12}: 1\right]$ and $\mathrm{M}$ is 83 . 
Figure 3.12: Barrier Option Prices for Two Assets(Brent Oil and Onatrio Gasoline)by using Bivariate Black-Scholes Model

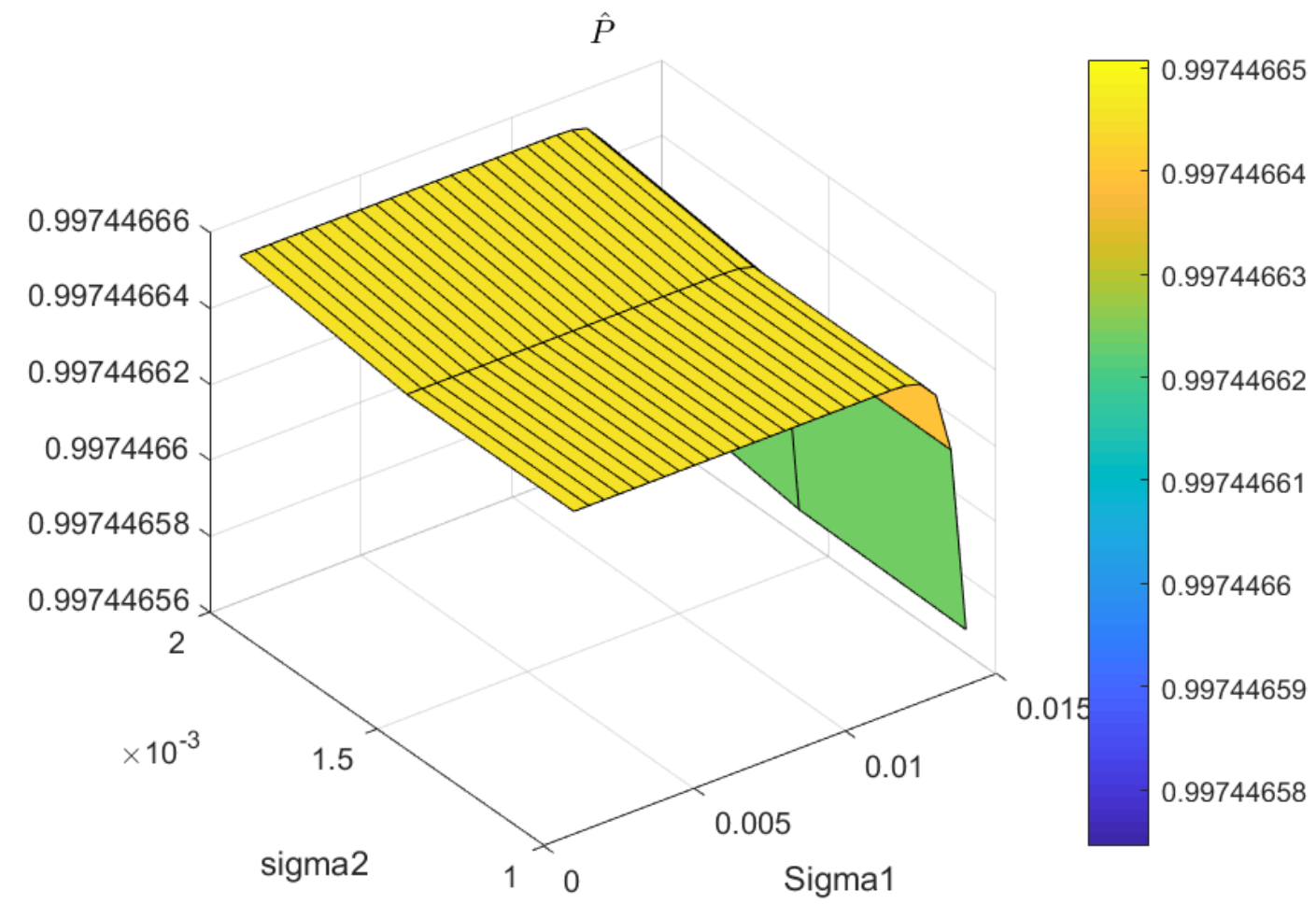

Barrier Prices Option: The 3-D graph shows the interaction between $\sigma_{\text {Brent-Oil }}$,

$\sigma_{\text {Ontrio-Gasoline }}$ and the price of the option for two assets using bivariate

Black-Scholes model simulation. The $\sigma_{\text {Brent-Oil }}$ is [0.001:0.001:0.002] and

$\sigma_{\text {Ontrio-Gasoline }}$ is [0.001:0.001:0.014]. 
Figure 3.13: Barrier Option Prices for Two Assets(WTI Oil and Onatrio Gasoline)by using Bivariate Black-Scholes Model

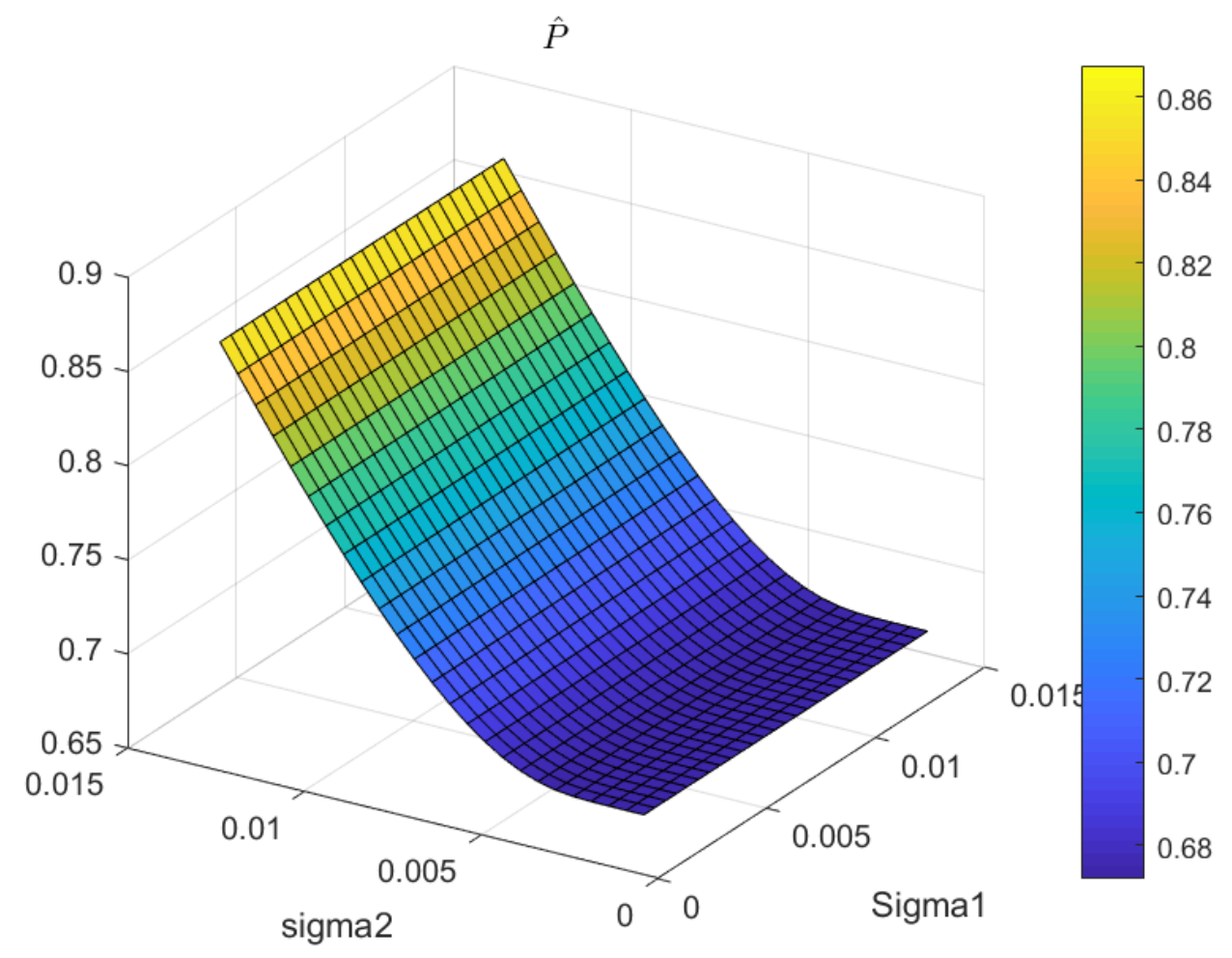

Barrier Prices Option: The 3-D graph shows the interaction between $\sigma_{\text {Brent-Oil }}$,

$\sigma_{\text {Ontrio-Gasoline }}$ and the price of the option for two assets using bivariate

Black-Scholes model simulation. The $\sigma_{W T I-O i l}$ is [0.001:0.001:0.002] and

$\sigma_{\text {Ontrio-Gasoline }}$ is [0.001:0.001:0.014]. 
Figure 3.14: Barrier Option Prices for Two Assets(Brent and WTI Oil)by using Bivariate Black-Scholes Model

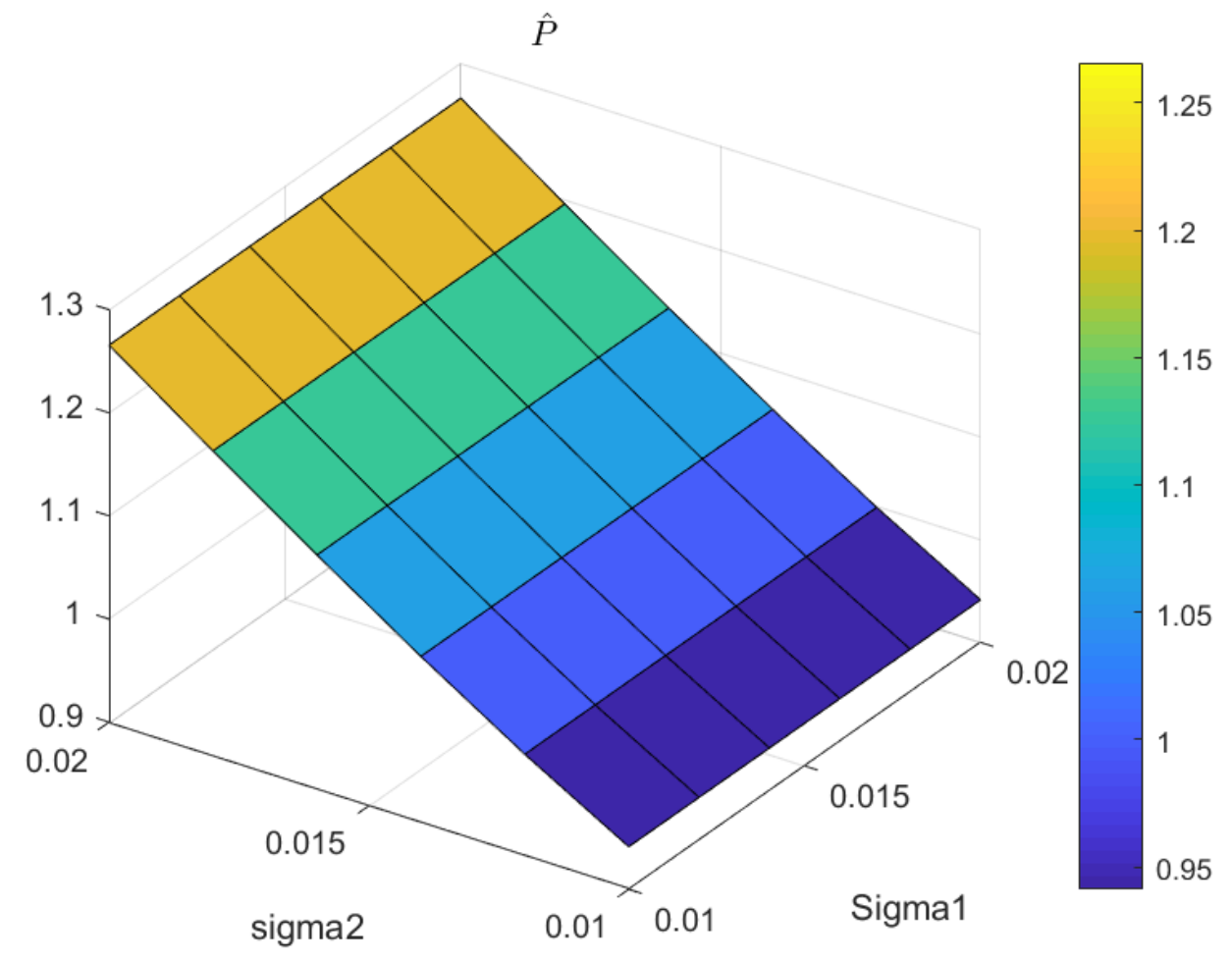

Barrier Prices Option: The 3-D graph shows the interaction between $\sigma_{\text {Brent-Oil }}$, $\sigma_{W T I-O i l}$ and the price of the option for two assets using bivariate Black-Scholes model simulation. The $\sigma_{\text {Brent-Oil }}$ is [0.01:0.001:0.02] and $\sigma_{W T I-O i l}$ is [0.01:0.01:0.02] 
Figure 3.15: Barrier Option Prices for Two Assets(Brent Oil and Ontario Gasolinel)by using Bivariate Black-Scholes Model

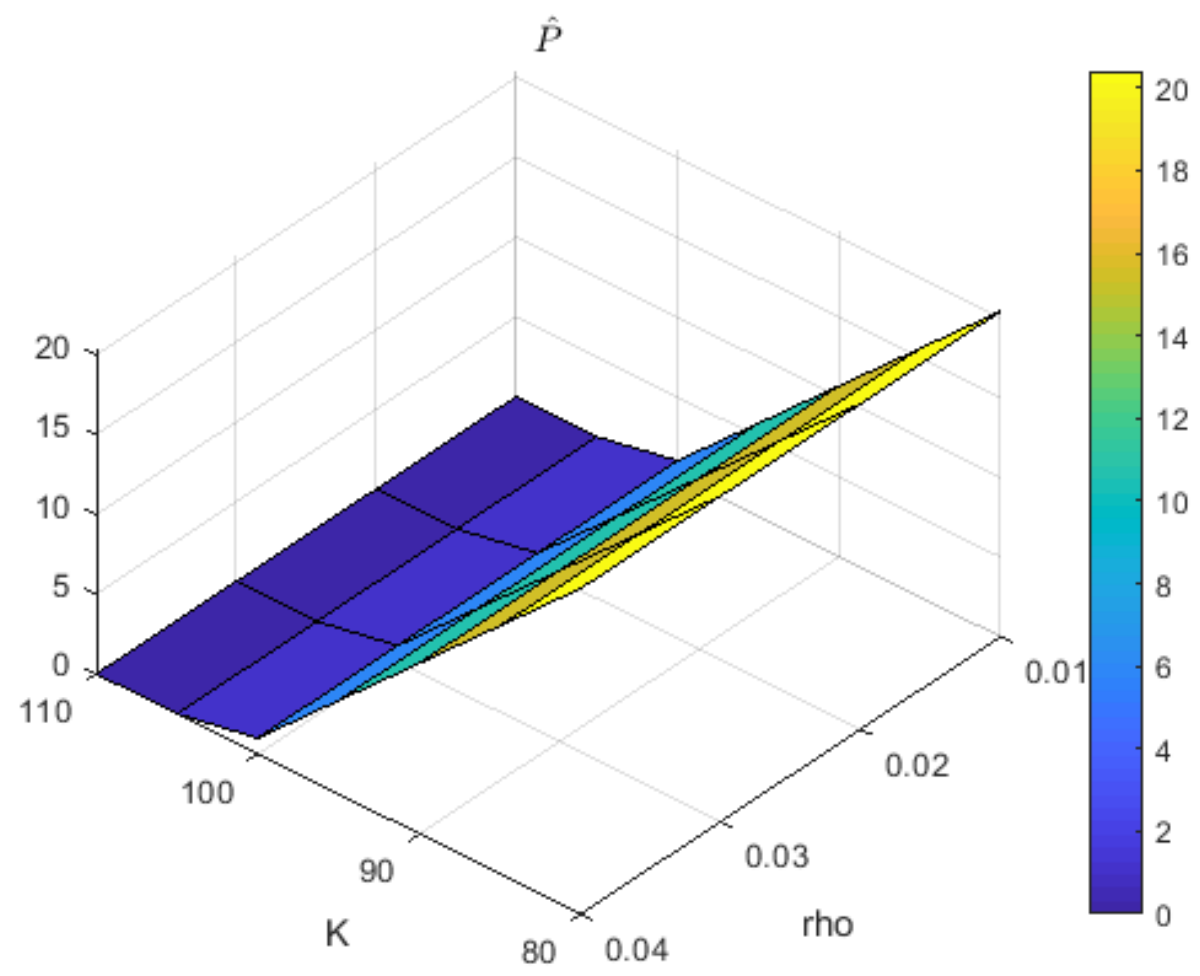

Barrier Prices Option: The 3-D graph shows the interaction between $\rho_{\text {Brent-Oil\&Ontario-Gasoline }}$ and the price of the option for two assets using bivariate Black-Scholes model simulation.The $\rho_{\text {Brent-Oil\&Ontario-Gasoline }}$ is [0.01:0.01:0.045] and $\mathrm{K}$ is [80:5:110]. 
Figure 3.16: Barrier Option Prices for Two Assets(WTI Oil and Ontario Gasolinel)by using Bivariate Black-Scholes Model

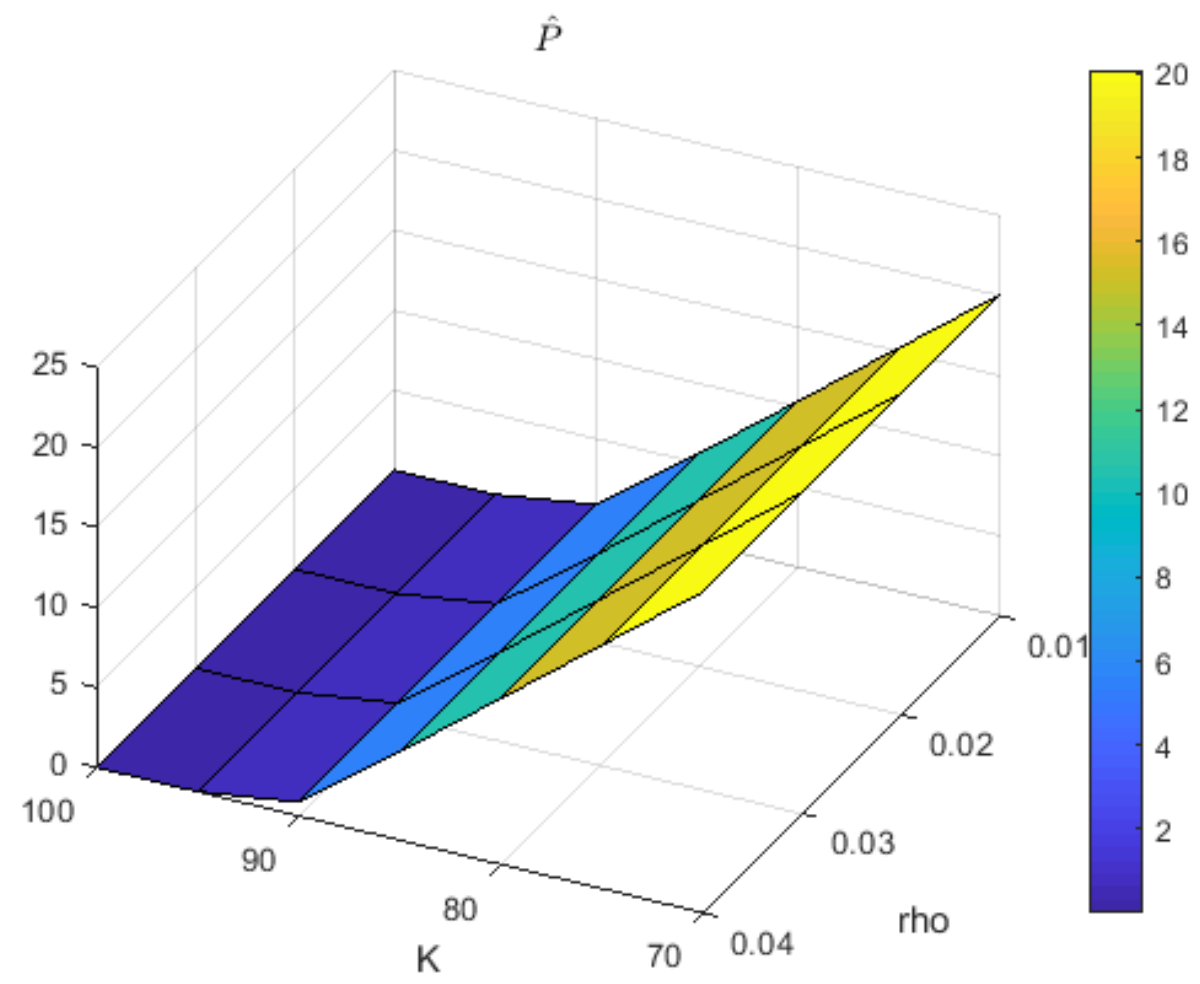

Barrier Prices Option: The 3-D graph shows the interaction between $\rho_{W T I-O i l \& O n t a r i o-G a s o l i n e}$ and the price of the option for two assets using bivariate Black-Scholes model simulation.The $\rho_{W T I-O i l \& O n t a r i o-G a s o l i n e}$ is [0.01:0.01:0.048] $\mathrm{K}$ is $[70: 5: 100]$. 
Figure 3.17: Barrier Option Prices for Two Assets(Brent and WTI Oil)by using Bivariate Black-Scholes Model

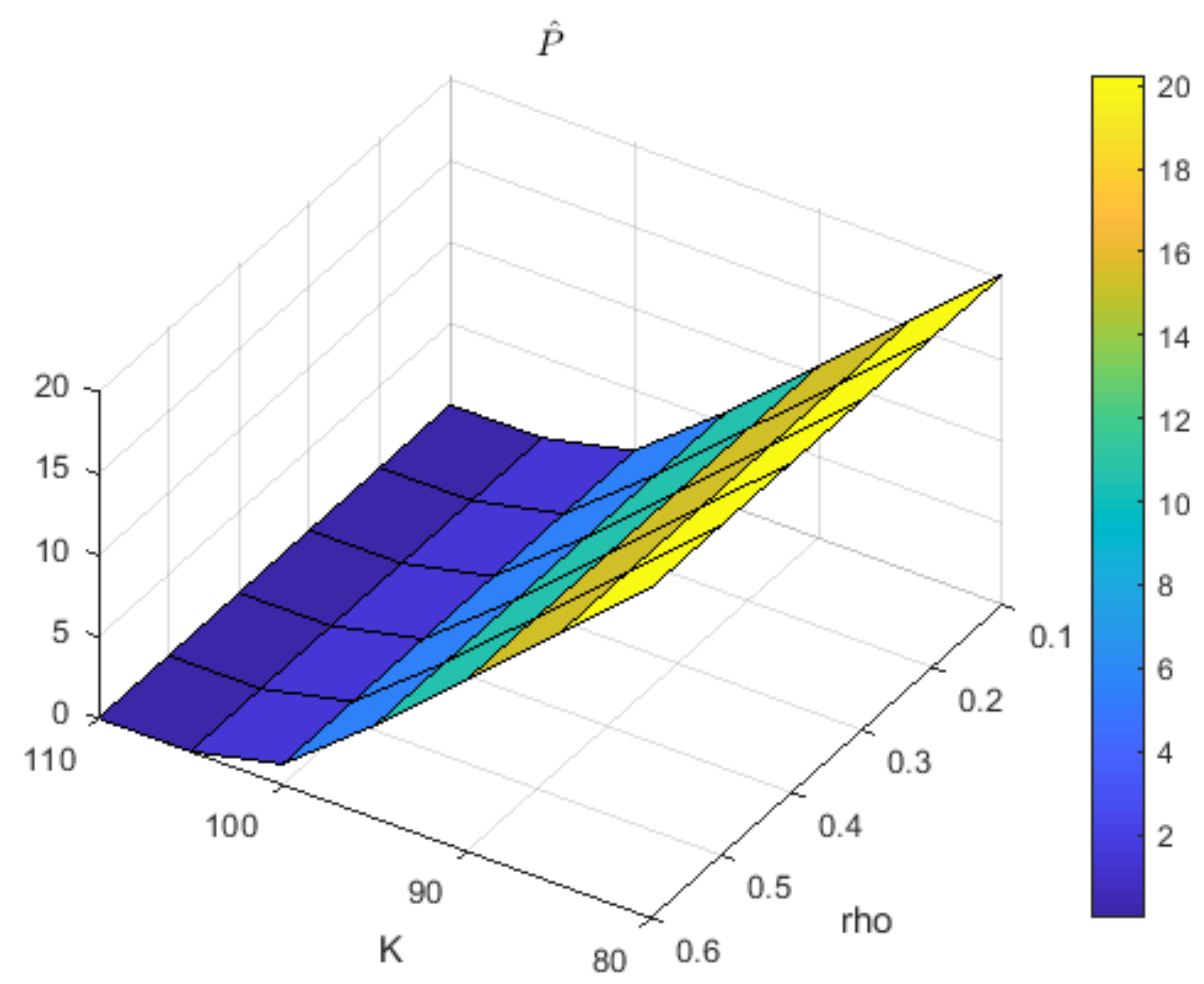

Barrier Prices Option: The 3-D graph shows the interaction between $\rho_{W T I-O i l}$, $\rho_{\text {Brent-Oil }}$ and the price of the option for two assets using bivariate Black-Scholes model simulation. The $\rho_{\text {Brent-Oil-Oil\&WTI-Oil }}$ is $[0.1: 0.1: 0.89] \mathrm{K}$ is $[80: 5: 110]$. 


\section{Chapter 4}

\section{PRICING BARRIER OPTION BASED ON TIME-CHANGED MODEL BY USING MONTE CARLO METHOD}

In this chapter, algorithms of more advanced and comprehensive univariate and bivariate Time-Changed model based on Inverse Gaussian are introduced to price the barrier option for same assets of chapter three.

To implement the introduced algorithms the Monte Carlo method is applied in simulation. The model verifiedd in simulation by implementing two main blocks Brownian motion and Inverse Gaussian process.

\subsection{Time-Changed Model}

\subsubsection{Univariate Time-Changed Model}

In order to price barrier option on oil and gas contracts, we assume a dynamic for the underlying commodities based on the spot price. The initial task is to define a model for the underlying commodities of spot price. Accordingly, we can define

$$
S_{t}=S_{0} \exp \left(Y_{t}\right)
$$


where $S_{t}, t \geq 0$ is the price for asset at time t, and $Y_{t}$ is the log-price. Moreover, we assume the log-price process $\left(Y_{t}\right)_{t \geq 0}$ verifies by

$$
Y_{t}=\sigma B_{A_{t}}+\mu A_{t}
$$

where $A_{t}$ is a subordinator with IG distribution. The process $\left(B_{t}\right)_{t \geq 0}$ is a Brownian motion.

An Normal Inverse Gaussian (NIG) $Y_{t}$ has characteristic exponent that is given by

$$
\phi_{Y_{t}}(u)=-a t \sqrt{-2 i v+b^{2}}-b
$$

where $v=u \mu+\frac{1}{2} \sigma^{2} u^{2} i$.

Notice that subordinators are positive processes which jumps the random times. We analyze Down-and-Out barrier options whose payoff at maturity $\mathrm{T}$, for a strike price $\mathrm{K}$, and Barrier $\mathrm{M}$ is given by:

$$
h_{B}\left(S_{t}\right)=\left(S_{T}-K\right)_{+} \mathbf{1}_{\left\{m_{T}^{s}>M\right\}} \quad 0 \leq t \leq T
$$

Finally the fair price of the contracts at time $\mathrm{T}$ with strike price $\mathrm{K}$ will be given by the following equation, which take the expectation of the payoff, and discount the payoff.

$$
P_{t}=e^{-r T} \mathbb{E}_{Q}\left[h_{B}\left(S_{t}\right)_{0 \leq t \leq T}\right]
$$

where $Q$ is an equivalent martingale measure and $\mathbb{E}_{Q}$ is the expectation with respect to the $\mathrm{Q}$. 


\subsubsection{Bivariate Time-Changed Model}

Along with univariate Time-Changed model, we need two-dimensional Time-Changed model to find the price for two assets in order to price the crack spread contracts and accordingly payoff of each commodity based on the spot price.Similar to univariate Time-Changed model begging with reproducing the spot prices for any assets, using the following equation

$$
S_{t}^{j}=S_{0}^{j} \exp \left(Y_{t}^{j}\right) \quad j=1,2
$$

where $\left(Y_{t}^{j}\right)$ is the log-price of asset $\mathrm{j}$

$$
Y_{t}^{j}=B_{A_{t}^{j}}^{j}+\mu_{j} A_{t}^{j} \quad j=1,2
$$

where

$$
A_{t}^{j}=X_{t}^{0}+C_{j} X_{t}^{j} \quad j=1,2
$$

and $\left(X_{t}\right)_{t \geq 0}=\left(X_{t}^{0}, X_{t}^{1}, X_{t}^{2}\right)_{t \geq 0}$ is a bivariate Levy process with independent components. Similar to univariate Time-Changed model, we have studied in down-and-out barrier options whose payoff at maturity $\mathrm{T}$, for a strike price $\mathrm{K}$, and barrier $\mathrm{M}$ is given by:

$$
h_{B}\left(S_{t}\right)=\left(S_{T}^{(1)}-S_{T}^{(2)}-K\right)_{+} \mathbf{1}_{\left\{m_{T}{ }^{(1)}-S_{(2)}>M\right\}} \quad 0 \leq t \leq T
$$

where

$$
m_{T}^{S^{(1)}-S^{(2)}}=\inf \left\{S_{t}^{(1)}-S_{t}^{(2)}\right\}, \quad 0 \leq t \leq T .
$$


We compute the price of contracts at maturity time, $\mathrm{T}$, with strike price, $\mathrm{K}$, by using the following equation at the end of simulation.

$$
\hat{P}_{t}=e^{-r(T-t)} \mathbb{E}_{Q}\left[h_{B}\left(S_{t}\right)_{0 \leq t \leq T}\right]
$$

where $r$ is interest rate.

\subsection{The Monte Carlo Method and Pricing Applications}

In this section we introduce Monte Carlo method for finding $P_{t}$. Since we take $\mathrm{m}$ is a very large number of paths of the stocks price process in this method then we are able to compute the payoff by using

$$
\hbar=\frac{1}{m} \sum_{i=1}^{m} h_{B}^{i}\left(S_{t}\right) \simeq \mathbb{E}_{Q}\left[h_{B}\left(S_{t}\right)_{0 \leq t \leq T}\right]
$$

The Monte Carlo Method is based on two foundations; the Law of Large Numbers (LLN), and the algorithm to generate positive random numbers. The LLN generally states that the average of the results from performing the same experiment a large

number of times m should be close to its expected value, and usually becomes closer as $m$ increases. See (Hazewinkel,2001). The Strong Law of Large Numbers(SLLN), and the Weak Law of Large Numbers(WLLN) are two versions of LLN which the Monte Carlo Method is convergent in both of them,therefore the SLLN is proposed below.

Proposition 4.1. The Strong Law of Large Numbers.

Let $X_{1}, X_{2}, \ldots, X_{n}$ be an independent and identically distributed sequence of random 
variables where $E\left[X_{1}\right]<\infty$ Then,

$$
\frac{1}{n} \sum_{n=1}^{\infty} X_{n} \rightarrow E\left[X_{1}\right]
$$

as $n \rightarrow \infty$. See(Prokhorov, )

The randomness comes from generating suitable random numbers using different generating techniques. In this method, we must generate a random number. Normal random numbers from Inverse Gaussian distribution will be created in TimeChanged model and will be used to determine the asset price and then the option price.

Both Univariate and bivariate Time-Changed model after generating Inverse Gaussian distribution, Brownian Motion will be generated for pricing asset(s) and then we applied Monte Carlo method for barrier option price.

The algorithms for each component of Time-Changed model to price option are given in next section. Figure(4.1) shows how the processes combine to have good output,price option. Uni and bi variate Inverse Gaussian and Brownian motion are initial simulation required. Then the Time-Changed model is used to simulate a trajectory the spot price for asset(s). At the end, the Monte Carlo is used to simulate the option price. For beginning, the algorithms for input Time-Changed model are discussed. Then followed by the algorithm of the Monte Carlo method. 


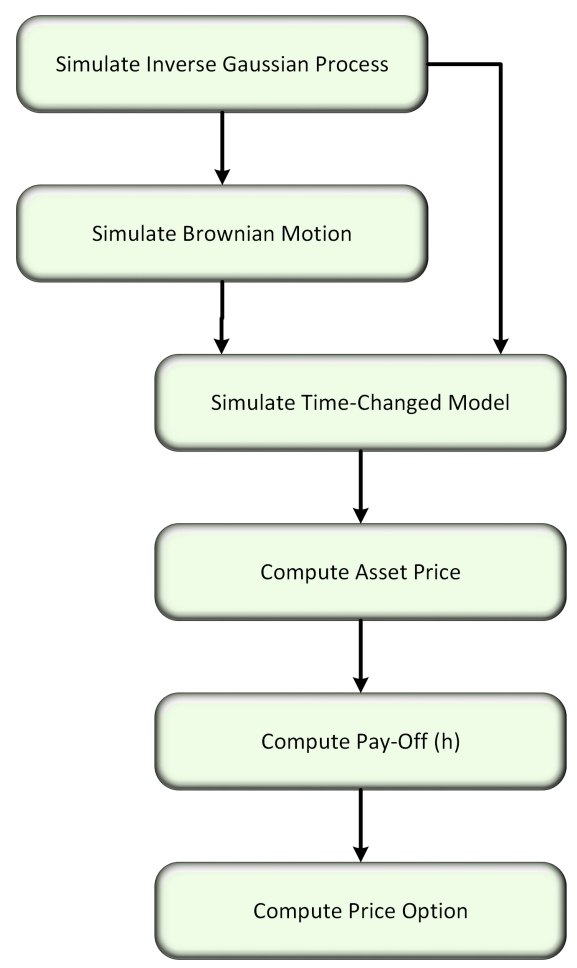

Figure 4.1: Flowchart Depicting the functions created to obtain the Price Option.

\subsection{Algorithm of Monte Carlo Output Functions}

Each process or function used in the methodology has its own respective algorithm which operates as the foundation to the software implementation. The algorithms for Brownian motion, Inverse Gaussian process and Monte Carlo method are explained for both univariate and bivariate in this section.

\subsubsection{Algorithm of Monte Carlo Under Univariate Time-Changed Model}

We begin with one dimensional Monte Carlo method by generating univariate Inverse Gaussian subordinator and Brownian Motion model. 


\section{Univariate Inverse Gaussian Subordinator}

Simulating subordinator $A_{t}$ is the first step of the Monte Carlo method simulation for time-changed model. The time-changed model proposed, uses an Inverse Gaussian subordinator $(\operatorname{IG}(a, b))$. We use the following algorithm known as the IG generator of Schucany and Haas.(Schucany\& Hass) .

\section{Algorithm(generating IG numbers with IG distribution):}

(1) Inputs $a>0, b>0$.

(2) Generate a standard Normal random number $v$.

(3) $y=v^{2}$.

(4) Set $x=\frac{a}{b}+\frac{y}{2 b^{2}}-\frac{\sqrt{4 a b y+y^{2}}}{2 b^{2}}$

(5) Generate a uniform random number $u$.

(6) If $u \leq \frac{a}{a+x b}$, then return the number $x$ as the IG(a,b) random number, else return $\frac{a^{2}}{b^{2} x}$ as the $\mathrm{IG}(\mathrm{a}, \mathrm{b})$ random number $A_{t}$. 
Numbers we generate the subordinator $\left(A_{t}\right)_{t \geq 0}$ by using the univariate Inverse Gaussian algorithm.

Inverse Gaussian Subordinator Simulation Result

Figure 4.2: Inverse Gaussian Subordinator Simulation Result

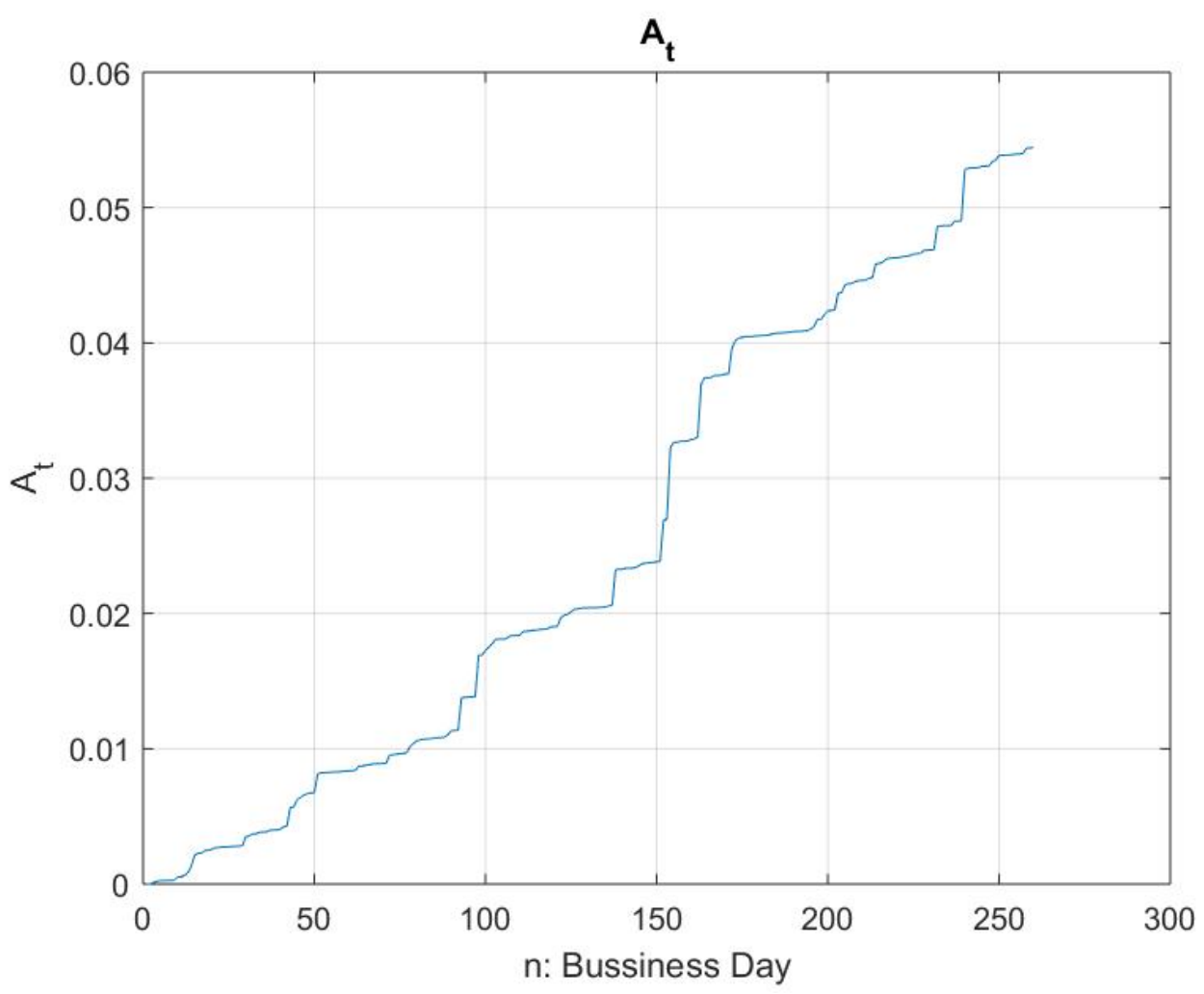

Figure 4.2 depicts an Inverse Gaussian process simulation fort $=[0, T]$, with $a=1$ and $b=20$. The parameters a and $\mathrm{b}$ represent the mean and standard deviation of the process, respectively. Evidently, the slope of the trajectory is varied. A steeper slope observed throughout the interval results in a greater change in the spot price. 


\section{Univariate Brownian Motion}

Simuating Brownian motion is the second step in the Monte Carlo simulation for univariate Time-Changed model. The goal is to simulate a Brownian motion on the interval $[0, T]$. For this simulation, we divided the interval into $n$ equal subintervals. Using the following algorithm:

\section{Algorithm}

(1) Input $T$ and $\Delta T$.

(2) The subintervals, $\mathrm{n}$, is calculated for the maturity period of $\mathrm{T}$, and increment of $\Delta T$ as $n=\left[\frac{T}{\Delta T}\right]$ and $\mathrm{n}$ is an integer.

(3) Using a univariate random number for generating univariate Brownian with mean of zero and the variance $\sigma$,

$$
B_{t} \sim N\left(0, \sigma A_{t}\right)
$$


Geometric Brownian Motion $B_{t}$ Simulation

Figure 4.3: Brownian Motion Simulation

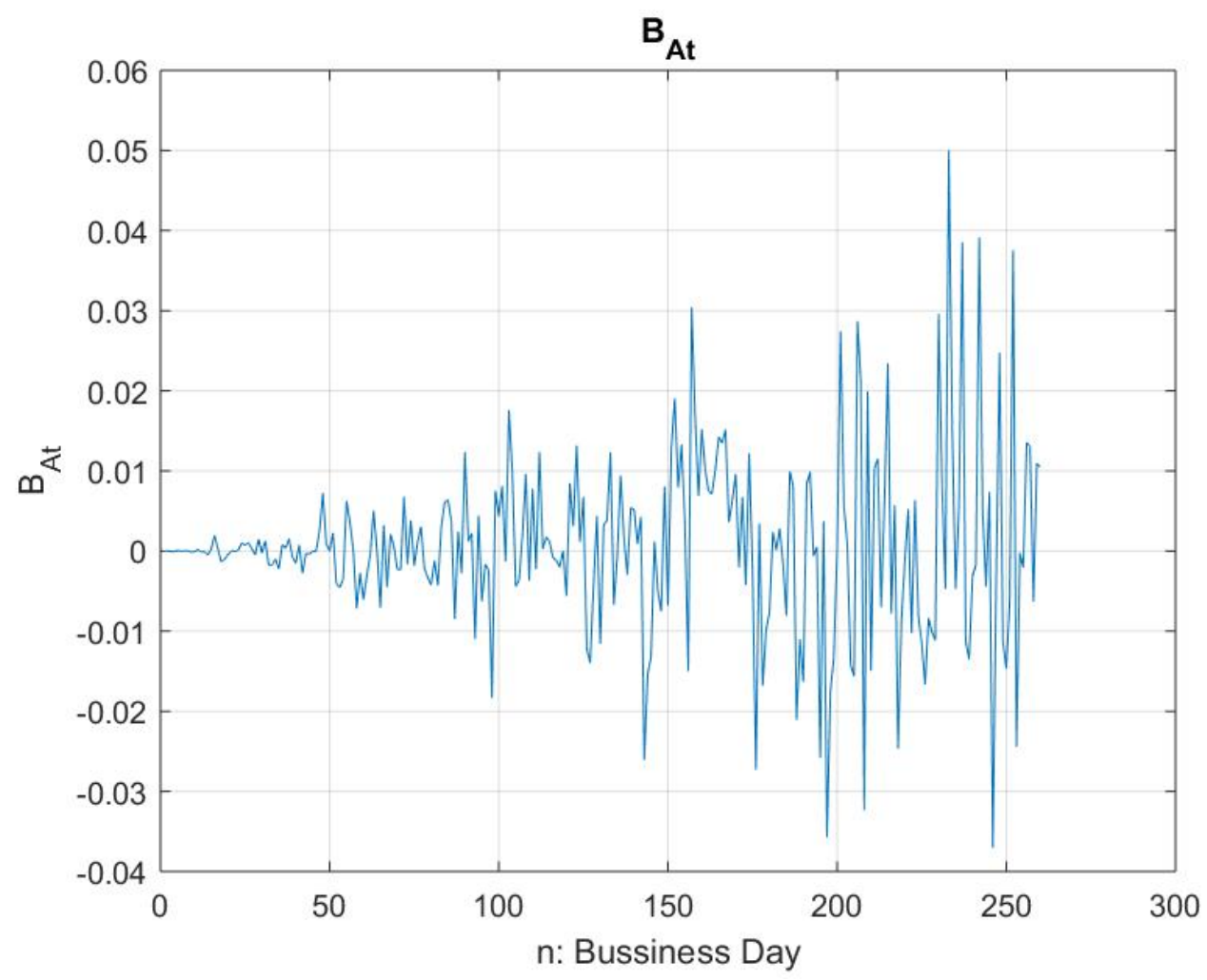

Figure 4.3 demonstrates the simulation of a trajectory Brownian motion, where $T$ is one year, $t \in[0, T], n=260$ mean zero, and $\sigma=0.2$. 


\section{Price Simulation}

Simulating the asset price is the last step before applying Monte Carlo method for Time-Changed model. IG subordinator and Brownian motion will be recall in asset price simulation.

\section{Algorithm}

(1) Input $A_{t}, B_{A_{t}}, \mathrm{~T}, S_{0}$, drift $(\mu)$ and volatility $(\sigma)$ of the asset.

(2) simulating $Y_{t}$ by using $A_{t}$ and $B_{A_{t}}$

$$
Y_{t}=\sigma B_{A_{t}}+\mu A_{t} \quad 0 \leq t \leq T .
$$

(3) Finding the spot price $\left(S_{t}\right)$ by

$$
S_{t}=S_{0} \exp \left(Y_{t}\right) \quad 0 \leq t \leq T .
$$


Figure 4.4: Spot Price for an Asset

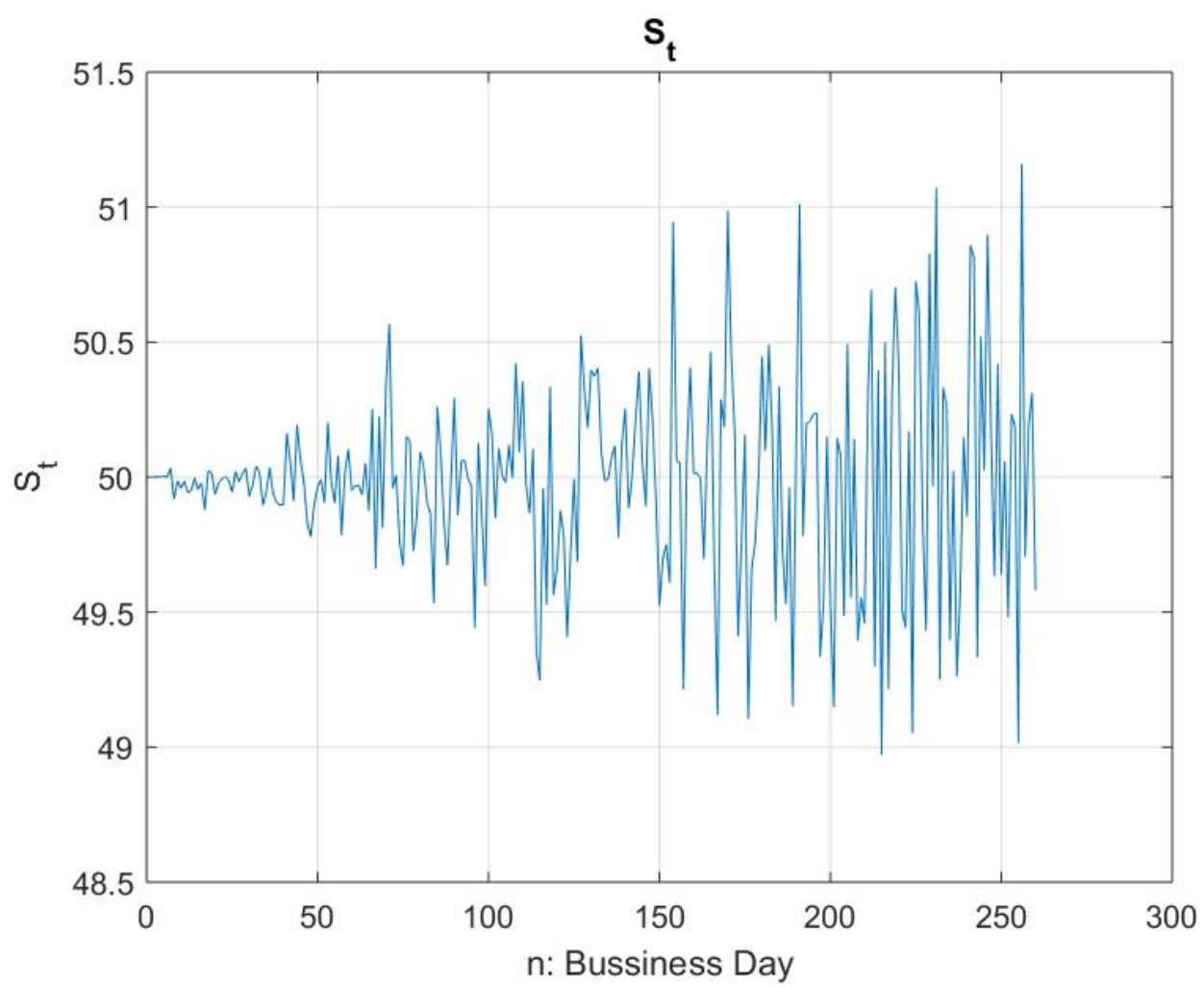

In the figure 4.4 spot price for an asset with $S_{0}=50$ over a period of $T=1$ year with 260 business day using Time-Changed model is simulated. 


\section{Univariate Monte Carlo Pricing}

The algorithm steps for pricing spark spread options using the Monte Carlo method is as below:

\section{Algorithm}

(1)Input strike price $(\mathrm{K})$, barrier (M), repetition (m) and Maturity time (T).

(2) Divide the interval $(0, T)$ into $n$ intervals with length of $\Delta t$, then simulate the sequence of prices:

$$
S_{\Delta t}, S_{2 \Delta t}, S_{3 \Delta t}, \ldots, S_{n \Delta t}=S_{T}
$$

(3) Compute $h_{B}\left(S_{t}\right)$

$$
h_{B}\left(S_{t}\right)=\left(S_{T}-K\right)_{+} \mathbf{1}_{\left\{m_{t}^{s}>M\right\}} \quad 0 \leq t \leq T .
$$

(4) Repeat 1 and 2 for $m$ times.

(5) Calculate $\hbar$

$$
\hbar=\frac{1}{m} \sum_{m=1}^{m} h_{B}\left(S_{t}\right)
$$

(6) Compute price option $\hat{P}$

$$
\hat{p}=\exp (-r T) \hbar
$$




\subsubsection{Algorithm of Monte Carlo for Bivariate Time-Changed Model}

In this subsection, bivariate Inverse Gaussian subordinator, Brownian Motion and Time-Changed model algorithms will be demonstrated and the algorithm for pricing the option price will be explain by Monte Carlo method at the end.

1. Multivariate Inverse Gaussian Subordinator For generating multivariate IG subordinator, we have the same 5 steps algorithm as a univariate IG subordinator. we have simulate two IG subordinator from step (6) as following:

\section{Algorithm:}

(1) Generate a standard Normal random number $v$.

(2) $y=v^{2}$.

(3) Set $x=\frac{a}{b}+\frac{y}{2 b^{2}}-\frac{\sqrt{4 a b y+y^{2}}}{2 b^{2}}$

(4) Generate a uniform random numberu.

(5) If $u \leq \frac{a}{a+x * b}$, then return the number $x$ as the $\operatorname{IG}(\mathrm{a}, \mathrm{b})$ random number, else return $\frac{a^{2}}{b^{2} * x}$ as the $\mathrm{IG}(\mathrm{a}, \mathrm{b})$ random number.

(6) Next, it is easy to simulate a sample path of an IG process $X_{\Delta t}^{j}$, where It follows an $I G(a t, b)$ law. We simulate the value of this process at time points $\{n \Delta t, \quad n=0,1, \ldots\}$ as follows. First generate independent $I G(a \Delta t, b)$ random numbers $\left\{i_{n}, \quad n \geq 1\right\}$, then

$$
X_{0}^{j}=0, \quad X_{n \Delta t}^{j}=X_{n-1 \Delta t}^{j}+i_{n} \quad n \geq 1, \quad j=1,2
$$

Where $i_{n}$ are $I G(a \Delta t, b)$ and $\Delta t=\frac{T}{n}$.

(7) finally we generate subordinator $A_{t}^{j}$ 
Figure 4.5: Bivariate Inverse Gaussian Subordinator

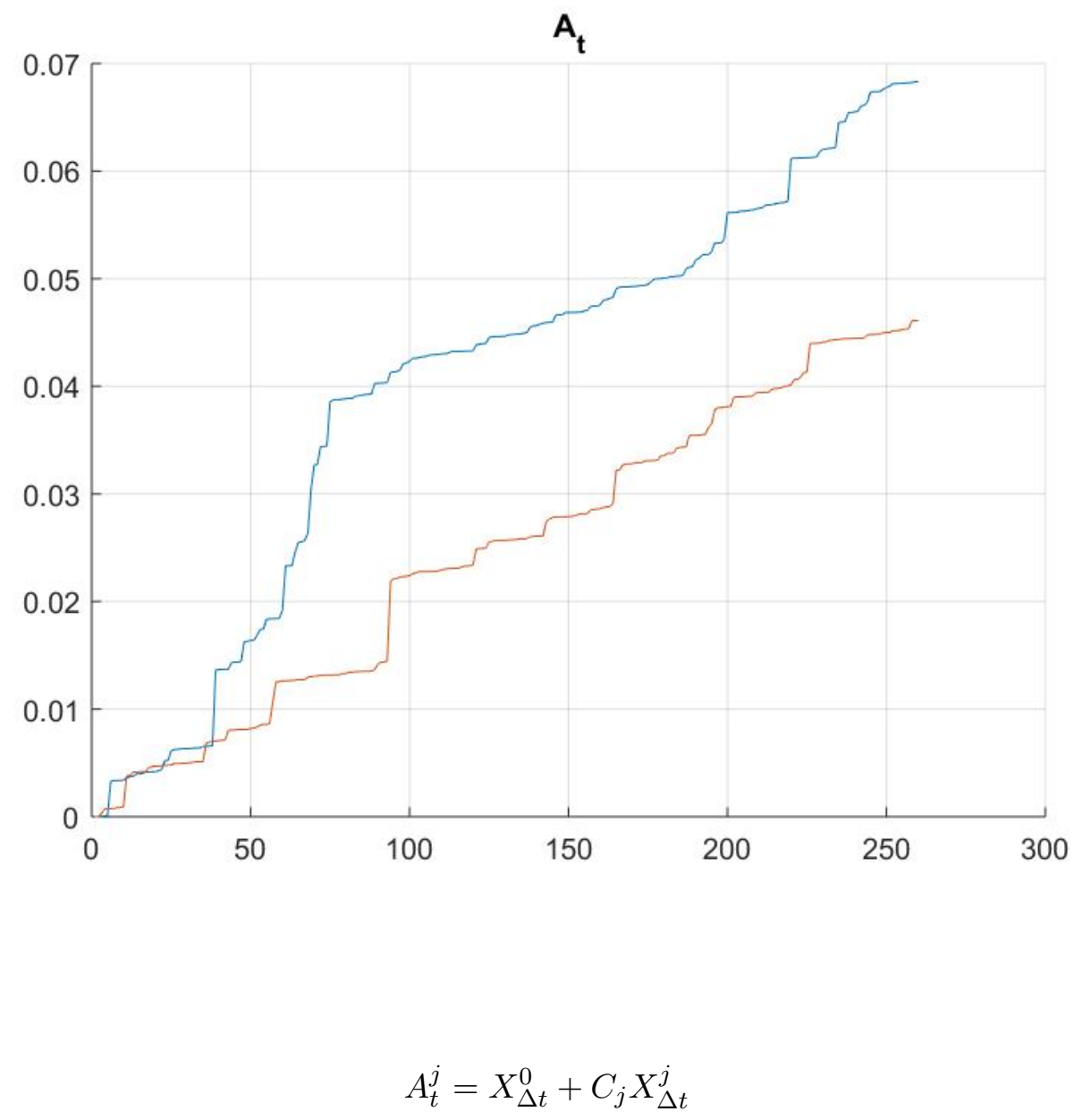

Where $C_{j}$ are constant number.

Figure 4.5: Inverse Gaussian subordinator simulated using $a=1, b=20$, maturity $T=1$ year and $\Delta t=1$ day 


\section{Brownian Motion Algorithm Algorithm}

(1) The subintervals, $n$, is calculated using the maturity period $T$, and increment $\Delta T$ as $n=\left[\frac{T}{\Delta T}\right]$ such that $\mathrm{n}$ is an integer

(2) The correlation matrix is created as

$$
\sum_{B}=\left[\begin{array}{cc}
\sigma_{1}^{2} & \rho \sigma_{1} \sigma_{2} \\
\rho \sigma_{1} \sigma_{2} & \sigma_{2}^{2}
\end{array}\right]
$$

We take $\rho=0$ that means, there is no correlation between $B_{t}^{1}, B_{t}^{2}$.

(3) The zero mean is given by

$$
\mu=(0,0)^{T}
$$

(4) Using a multivariate random number generator the two dimensional Brownian motion is simulated with zero mean and correlation $\sum_{B}$

$$
B_{A_{t}}^{j} \simeq N\left(0, A_{t}^{j} \sum_{B}\right)
$$


Figure 4.6: Bivariate Brownian Motion Simulation

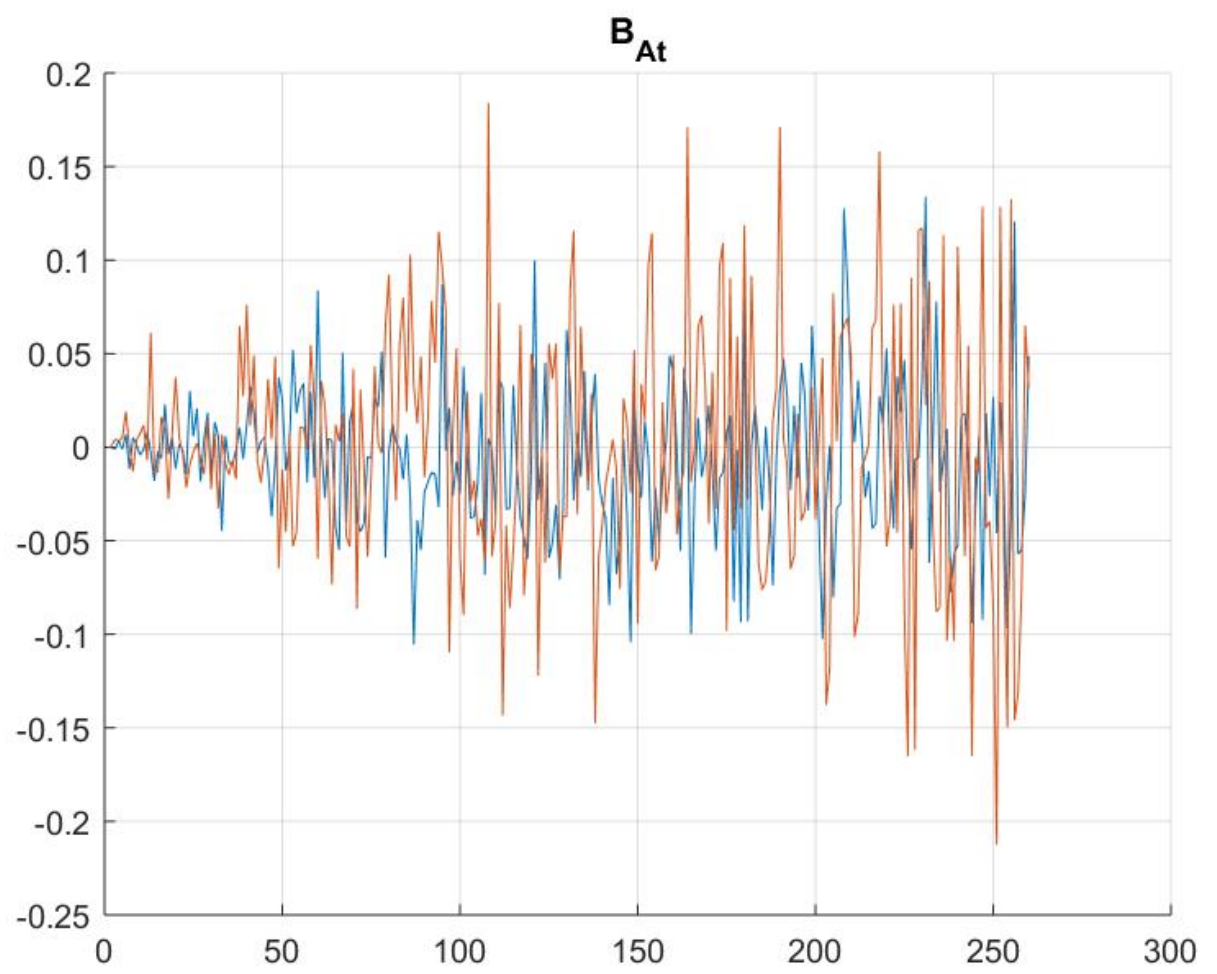


3. Multivariate Monte Carlo Pricing Algorithm The algorithm for pricing spark spread options using the Monte Carlo Simulation Method consists of the following steps:

(1) Divide the interval $(0, T)$ into $n$ intervals of length $\Delta t$ repeating the 2 simulations to obtain the $\mathrm{j}$-th simulation of the sequence of prices:

$$
S_{\Delta t}^{(j)}, S_{2 \Delta t}^{(j)}, S_{3 \Delta t}^{(j)}, \ldots, S_{n \Delta t}^{(j)}=S_{T}^{(j)}
$$

for $\mathrm{j}=1,2$

(2) Computing $h_{B}\left(S_{t}\right)$

$$
h_{B}\left(S_{t}\right)=\left(S_{T}^{(1)}-S_{T}^{(2)}-K\right)_{+} \mathbf{1}_{\left\{S_{T}^{(1)}-S_{T}^{(2)}>M\right\}} \quad 0 \leq t \leq T
$$

3. Repeat all these steps for $m$ times.

4. Calculating $\hbar$

$$
\hbar=\frac{1}{m} \sum_{p=1}^{m} h_{p}
$$

5. Computing $\hat{p}(t)$

$$
\hat{p}(t)=\exp (-r T) \hbar
$$

\subsection{Monte Carlo Pricing Computation}

This section presents the result procured from the methodology proposed to price barrier option. Three dimensional graphs have been used to present the impact the various parameters present in Time-Changed model. The Monte Carlo method for both univariate and bivariate Time-Changed model is used with $n=1000000$ runs 
for each graphs.

We need a drift $(\mu)$ and the volatility $(\sigma)$ for each commodities, same as the BlackScholes model. By using characteristic exponent function (4.1) for Time-Changed model and

$$
\begin{gathered}
\text { Mean }=\mathbb{E}\left(Y_{t}\right)=-i \phi^{\prime}(0)=\frac{a t \mu}{b} \\
\mathbb{E}\left(Y_{t}^{2}\right)=(-i)^{2} \phi^{\prime \prime}(0)=\frac{a t\left(\sigma^{2} b^{2}-\mu^{2}\right)}{b^{3}} \\
\operatorname{Var}\left(Y_{t}\right)=\mathbb{E}\left(Y_{t}^{2}\right)-\left(\mathbb{E}\left(Y_{t}\right)\right)^{2}=\frac{a t\left(\sigma^{2} b^{2}-\mu^{2}\right)}{b^{3}}-\frac{a^{2} t^{2} \mu^{2}}{b^{2}}
\end{gathered}
$$

we can find the drift and volatility for each asset from the empirical data that presented in table (1.2).

\begin{tabular}{ccccc}
\hline Series & $\mu-$ daily & $\sigma_{\text {daily }}$ & $\mu$-weekly & $\sigma_{\text {weekly }}$ \\
\hline \hline BRE Oil & 0 & 0.09 & 0.12 & 0.06 \\
WTI Oill & 0 & 0.1 & 0.09 & 0.07 \\
Ontario Gasoline & - & - & 0.052 & 0.07
\end{tabular}

Table 4.1: $\mu$ and $\sigma$ for all three assets in Time-Changed Model

\subsubsection{Monte Carlo Pricing under Univariate Time-Changed Model}

To observe the dynamics of the prices for the barrier option contracts under univariate Time-Changed model, Three dimensional graphs have been created. Figure (4.7), (4.8) and (4.9) describe the price of the barrier option for Brent oil, WTI 
oil and Ontario gasoline versus the Maturity time and strike price. As the strike price increase the option price reduce as we expected based on the payoff formula. The same happen when the maturity time increase, as seen in univariate BlackScholes model. All three graphs demonstrate sharp decrease in the option price when $K=\$ 100$ for Brent oil, $K=\$ 90$ for WTI oil and $K=\$ 70$ for Ontario gasoline. The time range taken for these graphs was $T=0.08,0.16,0.25,0.5,0.6,0.75$ and 1 year.

Figures (4.10), (4.11) and (4.12) show the interaction between tha barrier option price, strike price and the volatility $(\sigma)$ for all three commodities. Each of them $\sigma_{\text {BrentOil }}, \sigma_{W T I O i l}$ and $\sigma_{\text {OntarioGasoline }}$ is characterized as the coefficient volatility of Brownian motion process in Time-Changed model. In all commodities, very little alteration in the price option is happened with changing the commodities' volatility. The barrier option price is zero after initial strike price for each asset. This price is clarified by the payoff formula.

Figures (4.13), (4.14) and (4.15) show the interaction between the price for Downand-Out call barrier option and the Inverse Gaussian process parameters (a,b). Based on graphs, the payoff is zero most of the times and highest price is observed at lowest value for $b=10$ and largest value for $a=1$. When $b$ increases the price option declines very sharply to zero. 
Figure 4.7: Simulation of option price for Brent Oil in Univariate Time-Changed Model

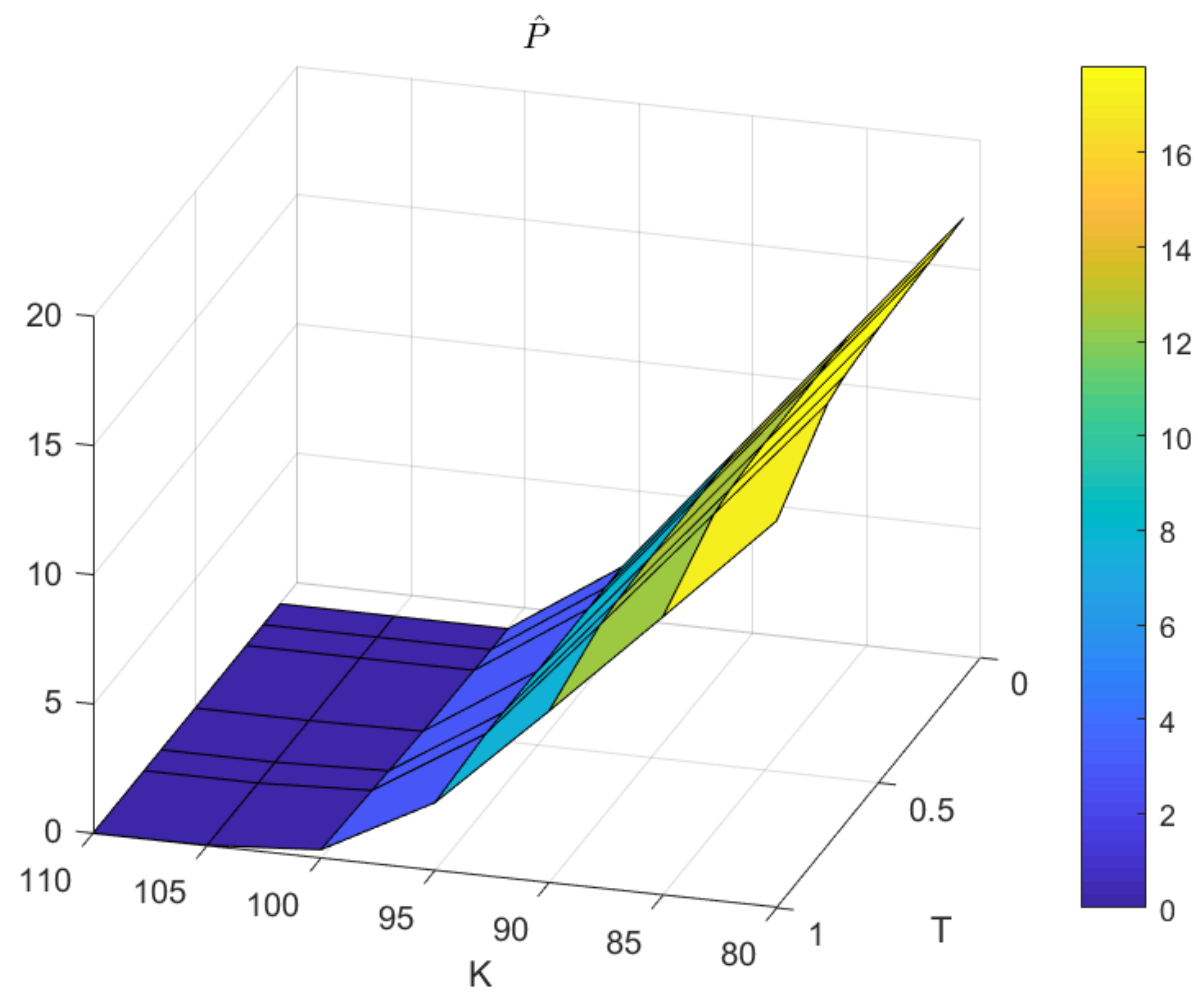

Prices for Brent oil: The 3-D graph shows the interaction between $\mathrm{T}$ ( maturity of the barrier option), $K($ Strike price of the barrier option) and the price of the option using univariate Time-Changed model simulation. The $\mathrm{K}$ is [80:5:110] and $\mathrm{T}$ is $\left[\frac{1}{12}: \frac{1}{12}: 1\right]$. 
Figure 4.8: Simulation of Option Price for WTI Oil in Univariate Time-Changed Model

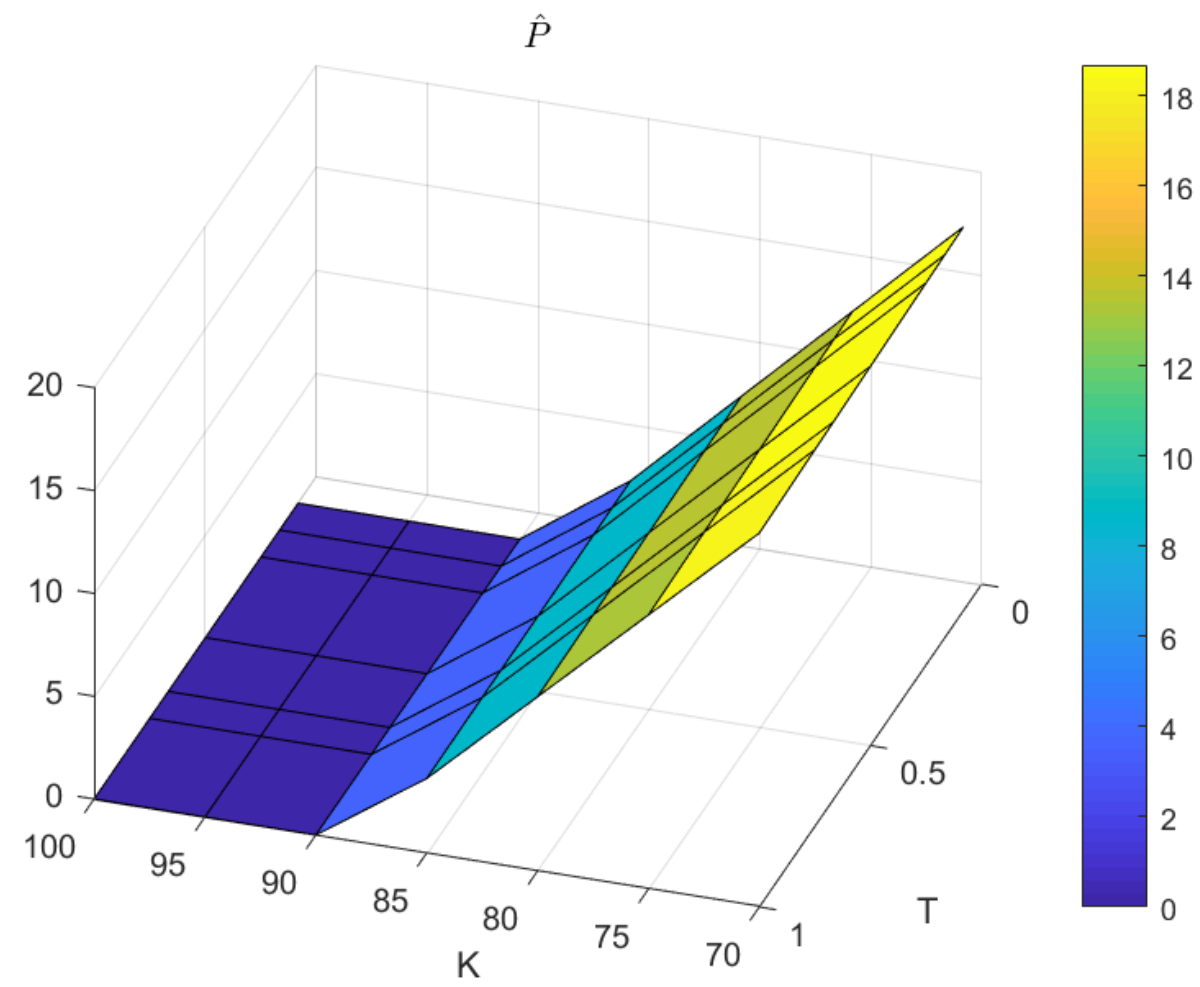

Prices for WTI oil: The 3-D graph shows the interaction between $\mathrm{T}$ ( maturity of the barrier option), $\mathrm{K}$ (Strike price of the barrier option) and the price of the option using univariate Time-Changed model simulation. The K is [70:5:100] and $\mathrm{T}$ is $\left[\frac{1}{12}: \frac{1}{12}: 1\right]$. 
Figure 4.9: Simulation of Option Price for Ontario Gasoline in Univariate TimeChanged Model

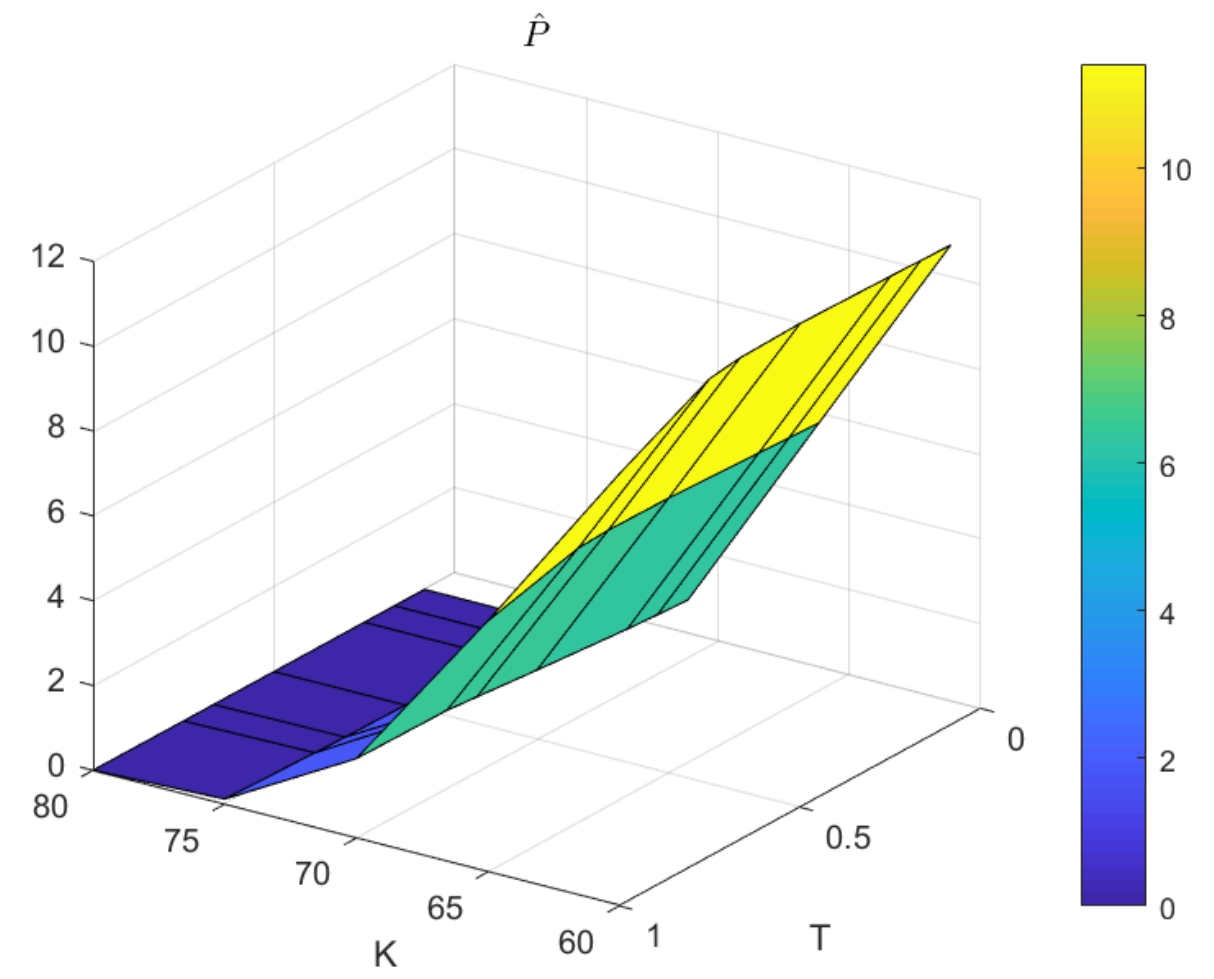

Prices for Ontario gasoline: The 3-D graph shows the interaction between $\mathrm{T}$ ( maturity of the barrier option), $\mathrm{K}$ (Strike price of the barrier option) and the price of the option using univariate Time-Changed model simulation. .The $\mathrm{K}$ is [60:5:90] and $\mathrm{T}$ is $\left[\frac{1}{12}: \frac{1}{12}: 1\right]$ 
Figure 4.10: Simulation of Option Price for Brent Oil in Univariate Time-Changed Model

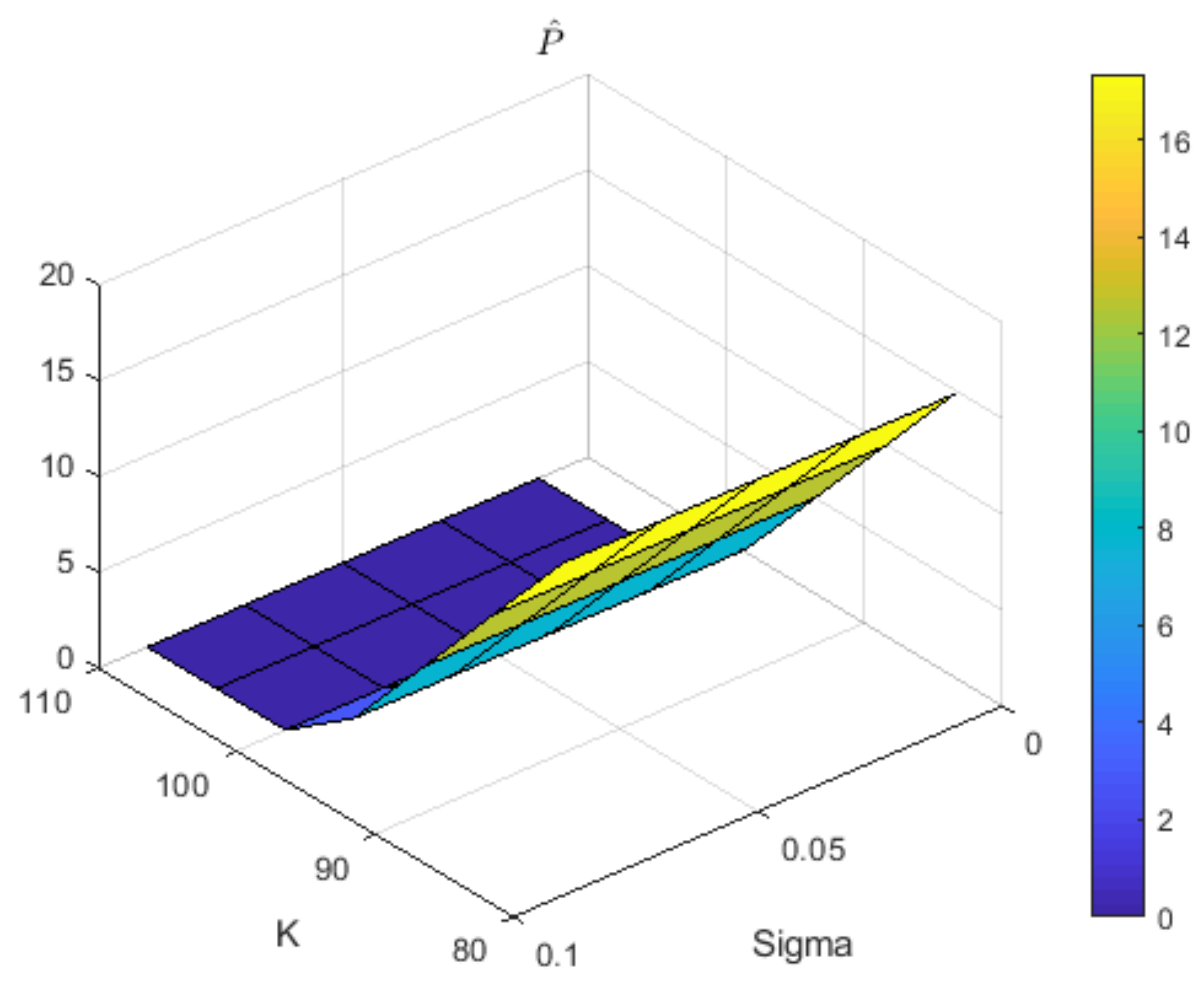

Prices for Brent oil: The 3-D graph shows the interaction between $\sigma_{\text {Brent-oil }}$, $\mathrm{K}$ (Strike price of the barrier option) and the price of the option using uni-variate Time-Changed model simulation. $\sigma_{\text {Brent-oil }}$ is [0.01:0.02:0.09]. 
Figure 4.11: Simulation of Option Price for Brent Oil in Univariate Time-Changed Model

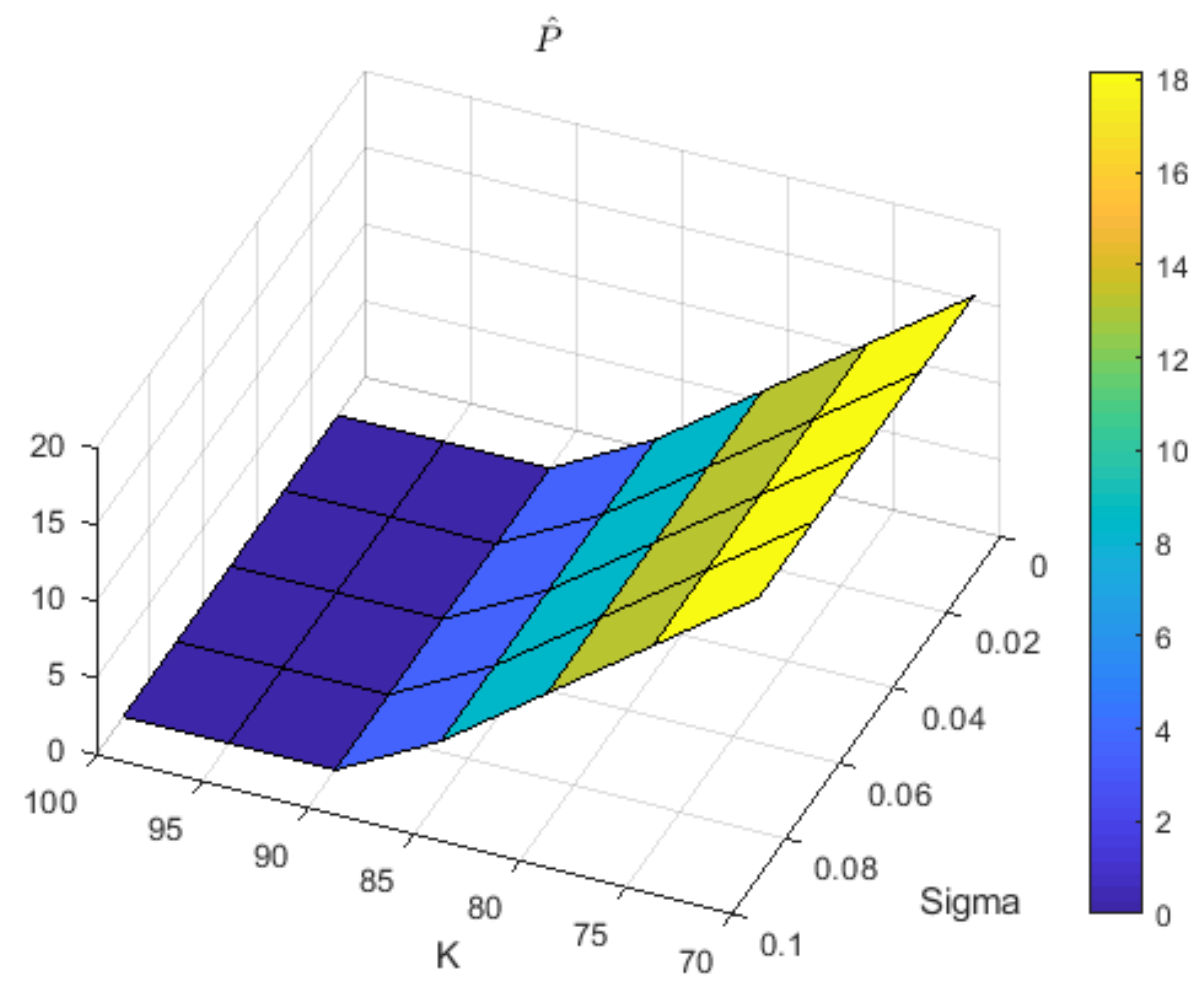

Prices for WTI oil: The 3-D graph shows the interaction between $\sigma_{W T I-o i l}$, $\mathrm{K}$ (Strike price of the barrier option) and the price of the option using uni-variate Time-Changed model simulation. $\sigma_{W T I-o i l}$ is [0.01:0.02:0.1]. 
Figure 4.12: Simulation of Option Price for Ontario Gasoline in Univariate TimeChanged Model

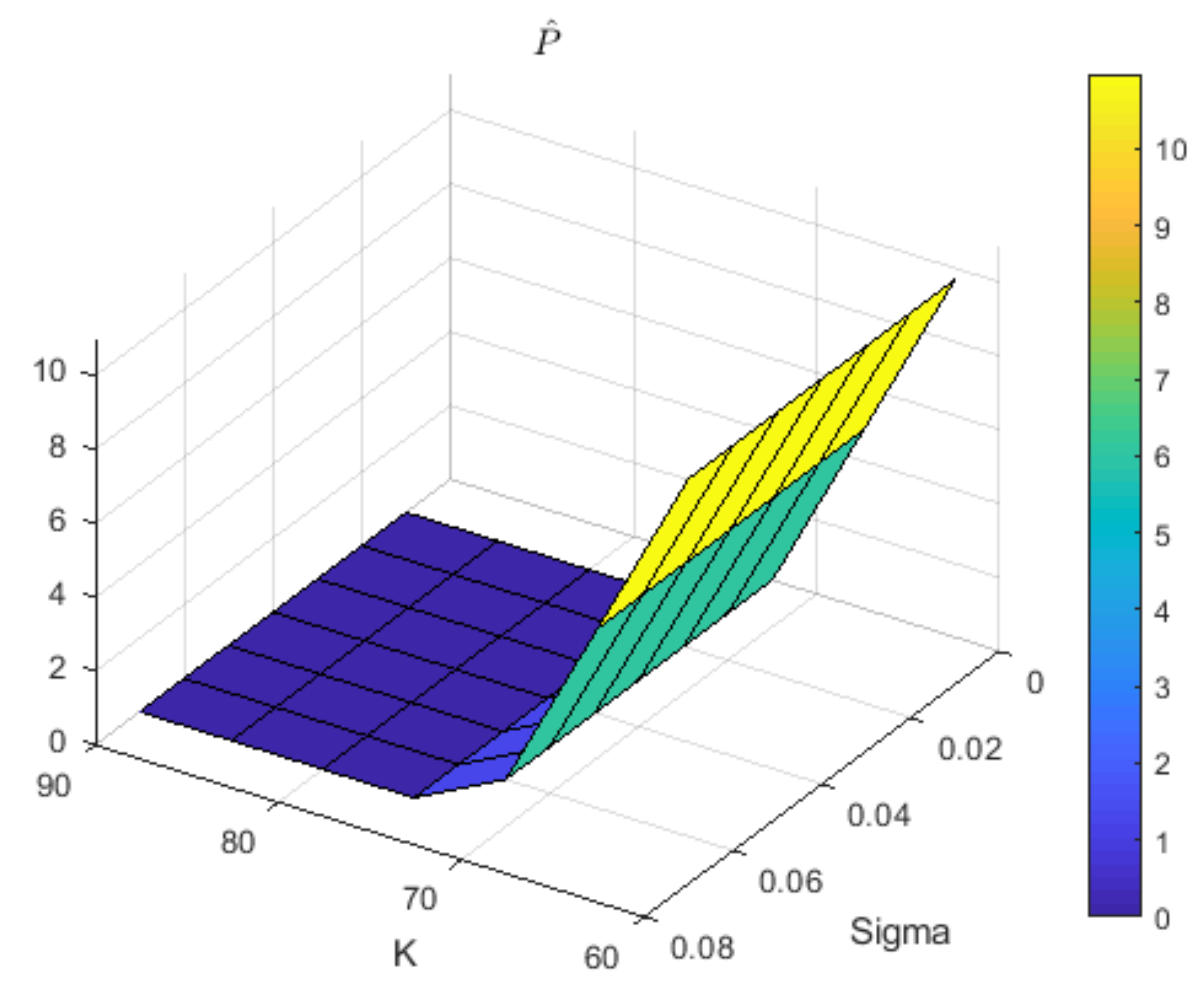

Prices for Ontario gasoline: The 3-D graph shows the interaction between $\sigma_{W T I-o i l}, \mathrm{~K}($ Strike price of the barrier option) and the price of the option using univariate Time-Changed model simulation. $\sigma_{\text {Ontario-Gasoline }}$ is [0.01:0.02:0.07]. 
Figure 4.13: Simulation of Option Price for Brent Oil in Univariate Time-Changed Model

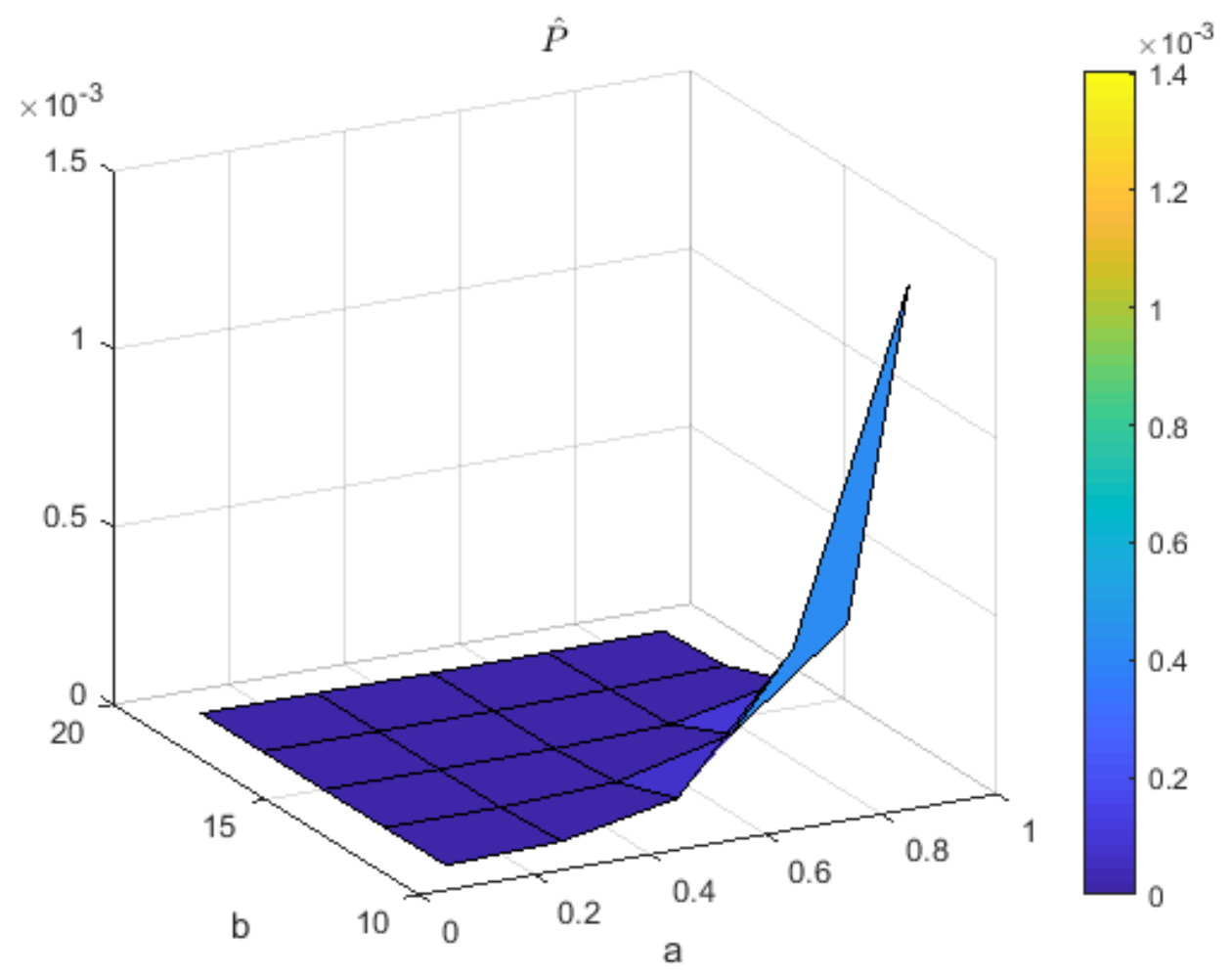

Prices for Brent oil: The 3-D graph shows the interaction between a, b and the price of the option using univariate Time-Changed model simulation.a was [0.01:0.01:1] and b was [11:2:20]. 
Figure 4.14: Simulation of Option Price for WTI Oil in Univariate Time-Changed Model

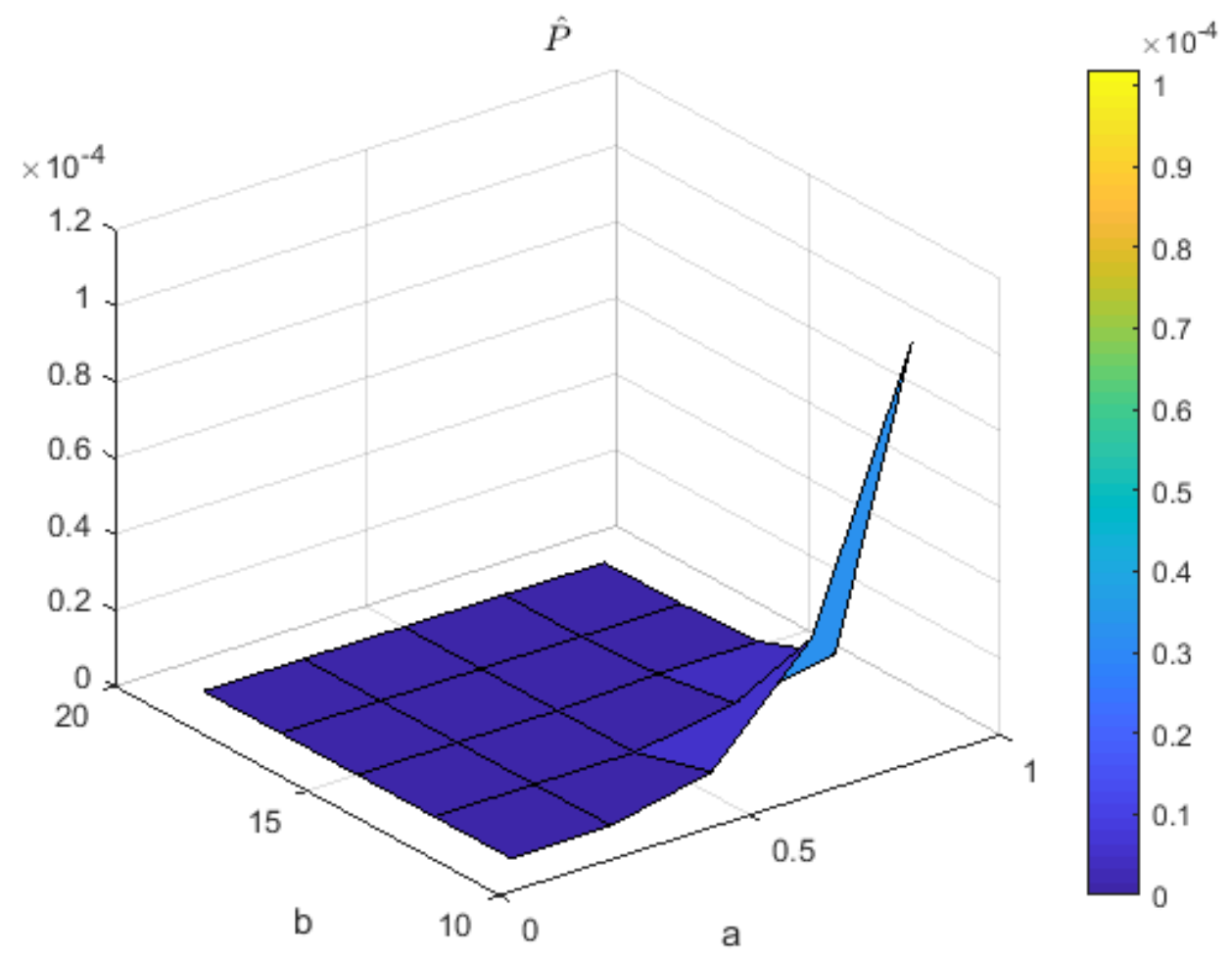

Prices for WTI oil: The 3-D graph shows the interaction between a, b and the price of the option using univariate Time-Changed model simulation. a was [0.01:0.01:1] and b was [11:2:20]. 
Figure 4.15: Simulation of Option Price for Ontario Gasoline in Univariate TimeChanged Model

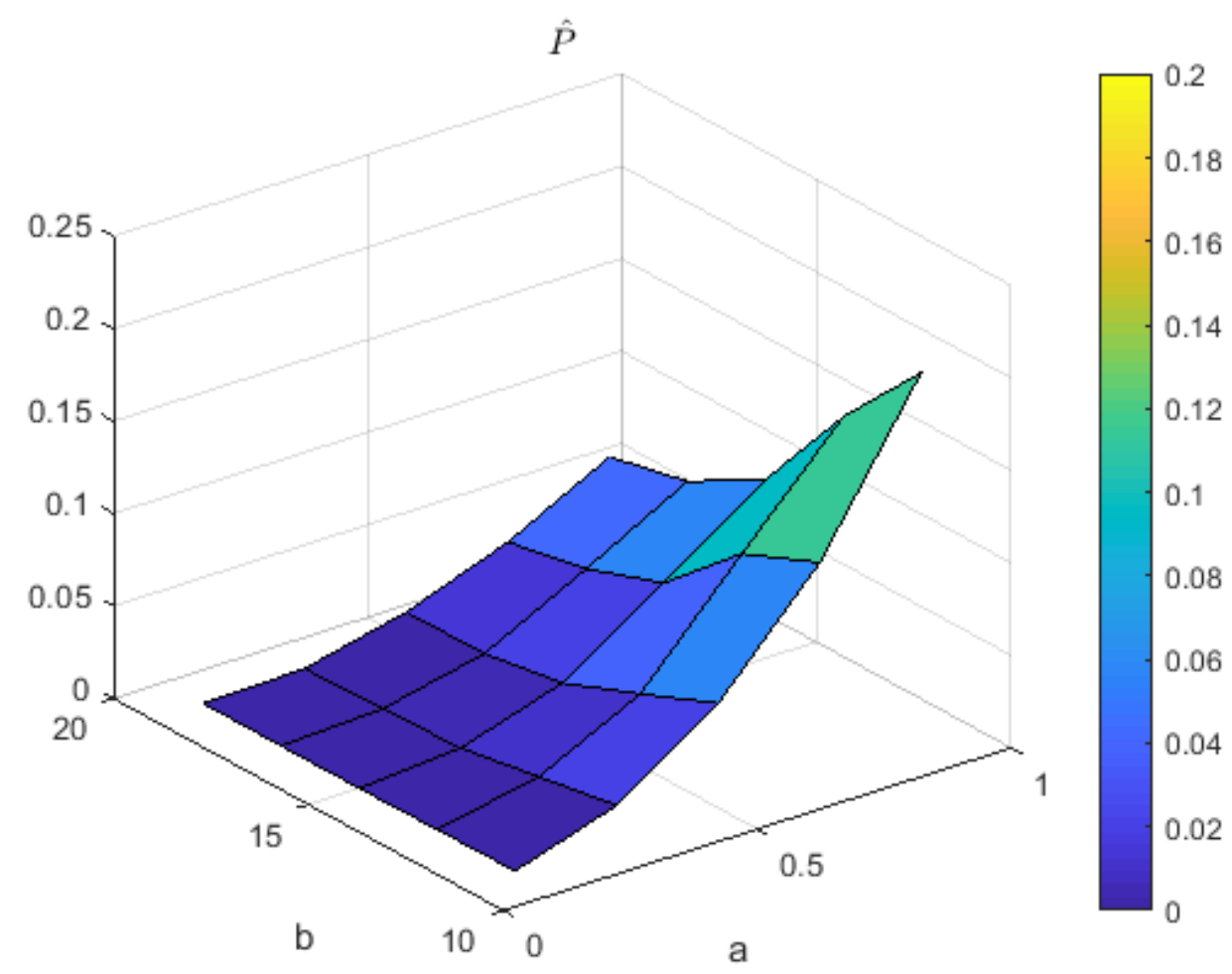

Prices for Ontario gasoline oil: The 3-D graph shows the interaction between a, b and the price of the option using univariate Time-Changed model simulation. a was [0.01:0.01:1] and b was [11:2:20]. 


\subsubsection{Monte Carlo Pricing under Bivariate Time-Changed Model}

To observe the dynamics of the prices for the barrier option contracts under bivariate Time-Changed model, Three dimensional graphs have been created. Figures (4.16), (4.17) and (4.18) display the difference in barrier option price between Ontario gasoline and Brent oil using Bivariate Time-Changed model. Figure (4.16) shows that by increasing $\mathrm{Ts}$, the option price increase drastically at first for small Ts and then increases very slowly like going to saturation for larger Ts between 0.5 to 1 year. It also shows that changing $\mathrm{K}$ from 1 to 5 does not affect that much on option price. Figure (4.17) shows that increasing $\sigma_{\text {OntarioGasoline }}$ and decreasing

$\sigma_{\text {Brentoil }}$ increases bivariate barrier option Price. Figure (4.18) shows that increasing $a_{0}$ and decreasing $b_{0}$ will increase price where the maximum price of around 32.2 is reached at $a_{0}=0.1$ and $b_{0}=20$.

Figures (4.19), (4.20) and (4.21) demonstrate the difference in barrier option price between Ontario gasoline and WTIl using Bivariate Time-Changed model. Figure (4.19) proves that the bivariate barrier option price decreases by increasing Time and strike price where the lowest price happens at maximum $\mathrm{K}$ of 5 and $\mathrm{T}$ equal to one year. Figure (4.20) shows that for bivariate barrier option changing $\sigma_{1}$ and $\sigma_{2}$ of both assets does not change the price that much.Figure (4.21) the price is less sensitive to Inverse Gaussian parameters $a_{0}$ and $b_{0}$. Where the price variation is small around 18.2-18.5 and the minimum price is reached when we have $a_{0}=0.1$ and $b_{0}=20$. 
Figures (4.22), (4.23) and (4.24) show the difference in barrier option price between Brent and WTI oil using Bivariate Time-Changed model. Similar to figure (4.19) ,figure (4.22) also shows that barrier option price increases linearly by decreasing K while the maturity time does not affect that much on price. Figures (4.23) simulation results show that both Inverse Gaussian parameters $a_{0}$ and $b_{0}$ have impact on price, where increasing both of them decreases the price and decreasing them increased the price and maximum price of around 5.5 is reached at $a_{0}=0.1$ and $b_{0}=20$. 
Figure 4.16: Bivariate Simulation of Barrier Option Price for Ontario Gasoline and Brent Oil in Bivariate Time-Changed Model

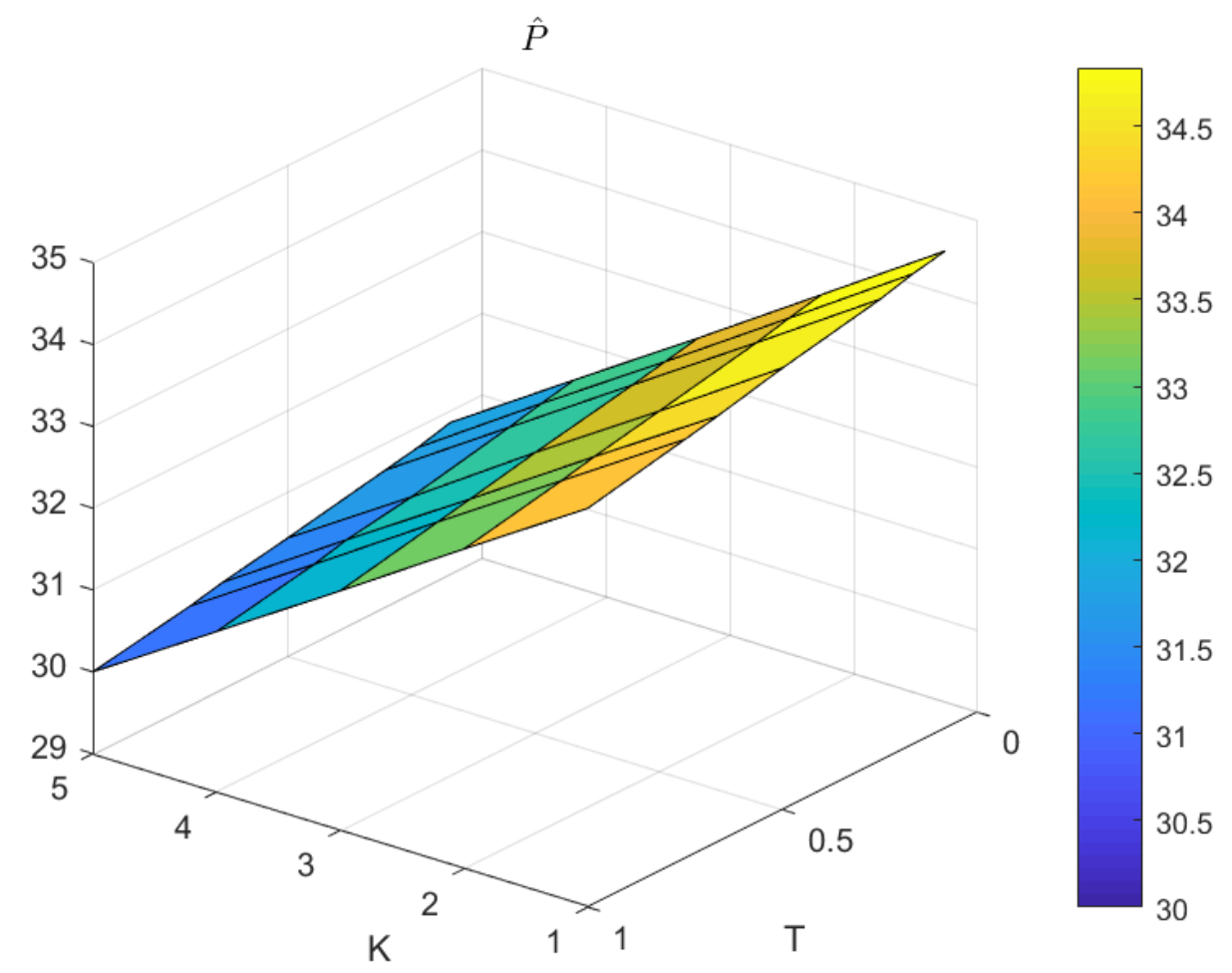

The graph shows bivariate barrier option prices change versus $\mathrm{T}$ ( maturity time of the barrier option per year), K(Strike price of the barrier option) and the price of the option based on bivariate Time-Changed model simulation. The $\mathrm{K}$ is [1:1:5] and $\mathrm{T}$ is $\left[\frac{1}{12}: \frac{1}{12}: 1\right]$. 
Figure 4.17: Simulation of Option Price for Ontario Gasoline and Brent Oil in Bivariate Time-Changed Model

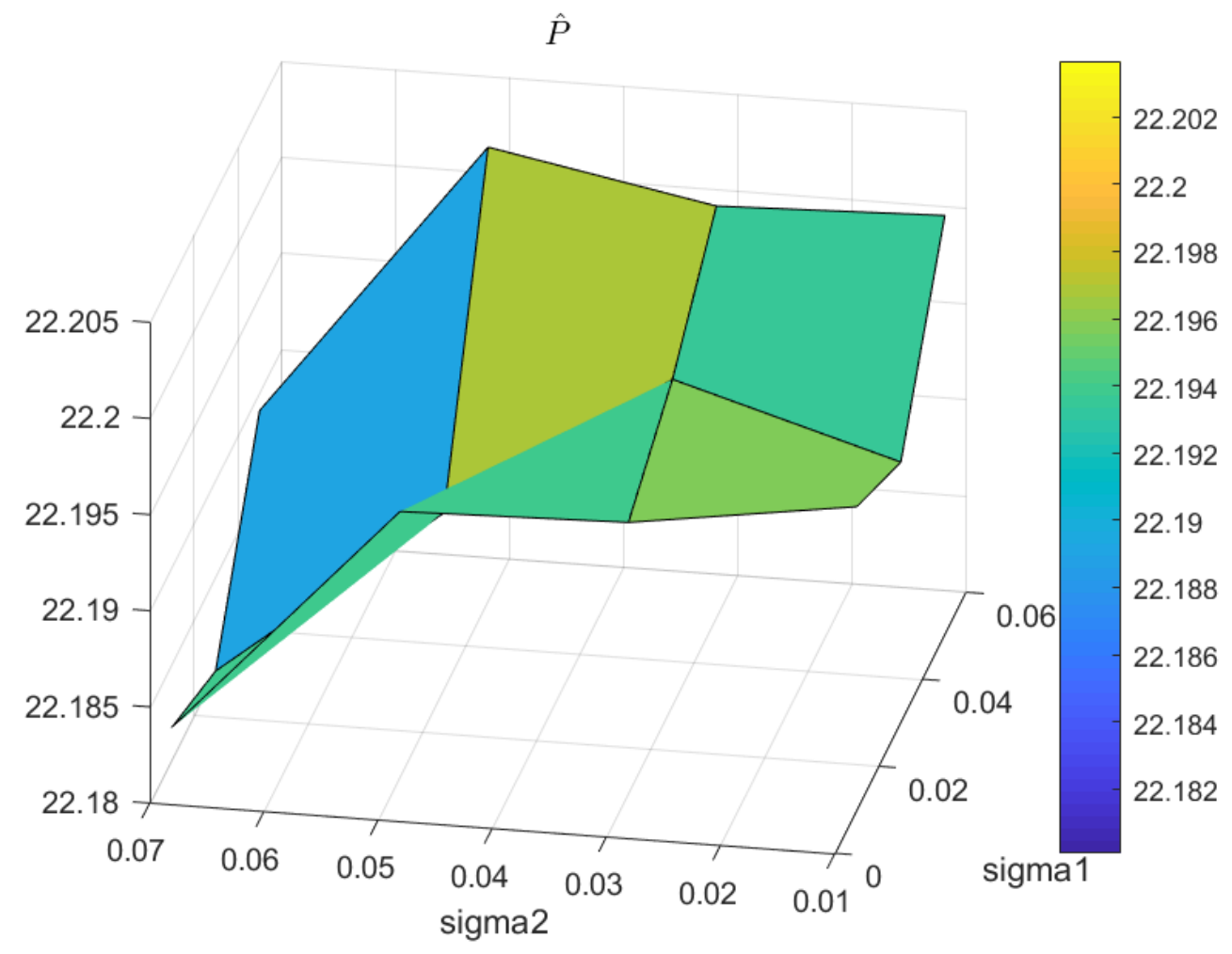

The graph depicts the effect of $\sigma_{\text {OntarioGasoline }}$ and $\sigma_{\text {Brentoil }}$ and variations on the price of the option using bivariate Time-Changed model simulation. $\sigma_{\text {BrentOil }}$ is $[0.01: 0.02: 0.06]$ and $\sigma_{\text {OntarioGasoline }}$ is $[0.01: 0.02: 0.07]$ 
Figure 4.18: Simulation of Option Price for Ontario Gasoline and Brent Oil in Bivariate Time-Changed Model

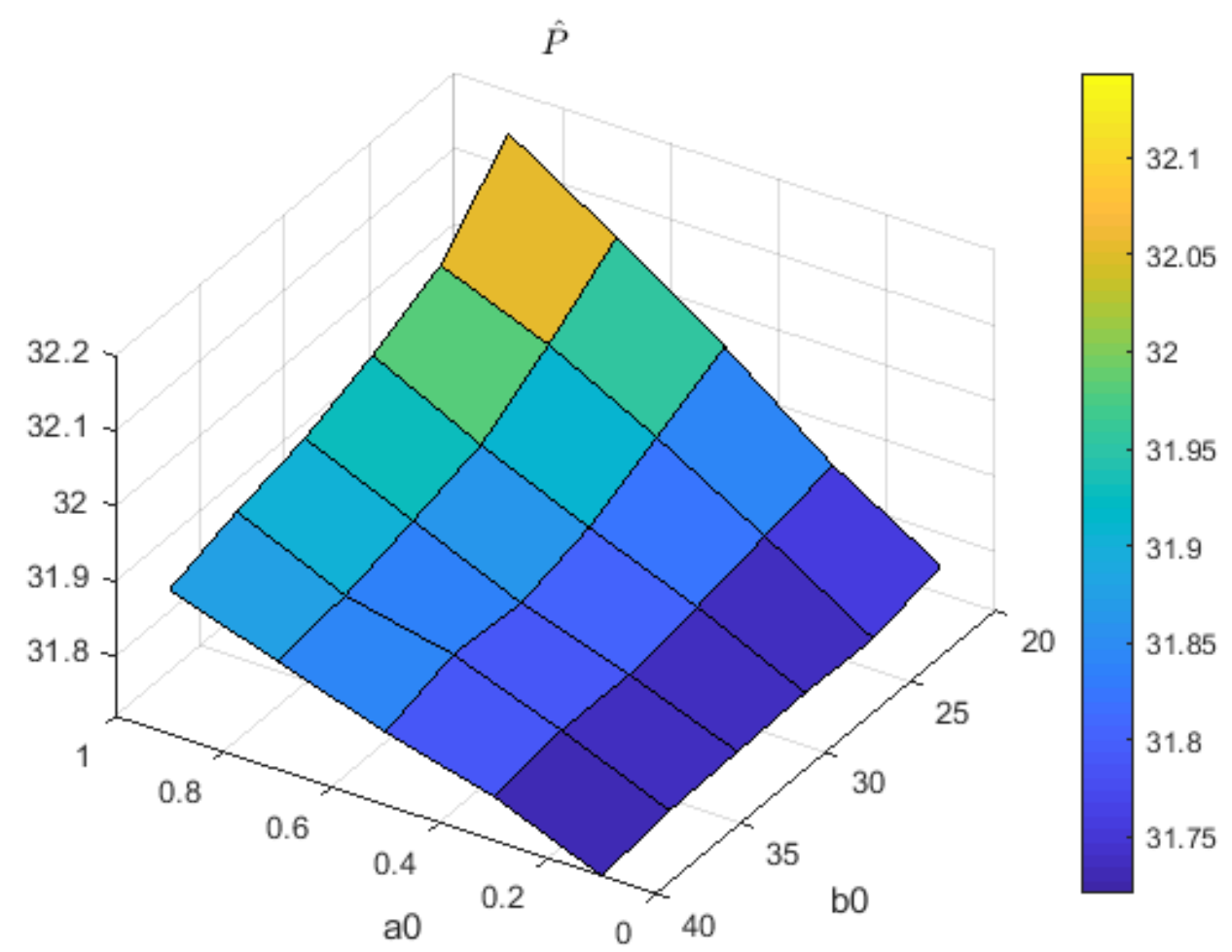

The 3-D graph displays the barrier option price variation with respect to the parameters of $I G\left(a_{0}, b_{0}\right.$ using bivariate Time-Changed model simulation. $a_{0}$ and $b_{0}$ are $[0.1: 0.2: 1]$ and $[20: 2: 40]$ respectably. 
Figure 4.19: Bivariate Simulation of Barrier Option Price for Ontario Gasoline and WTI Oil using Time-Changed Model

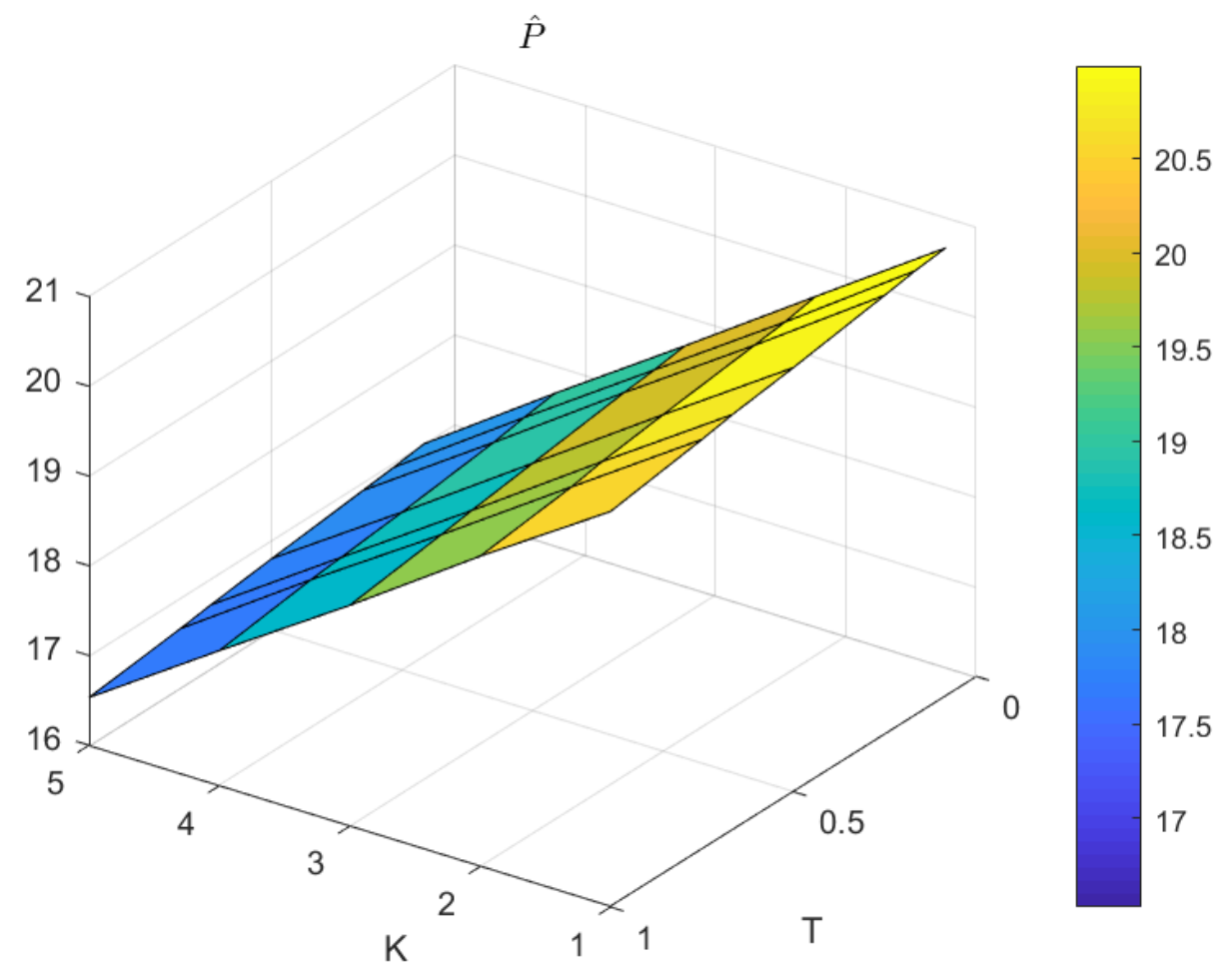

The graph shows the interaction between $\mathrm{T}$ ( maturity of the barrier option), $\mathrm{K}$ (Strike price of the barrier option) and the price of the option using bivariate Time-Changed model simulation. The $\mathrm{K}$ is $[1: 1: 5]$ and $\mathrm{T}$ is $\left[\frac{1}{12}: \frac{1}{12}: 1\right]$. 
Figure 4.20: Simulation of Option Price for Ontario Gasoline and WTI Oil in Bivariate Time-Changed Model

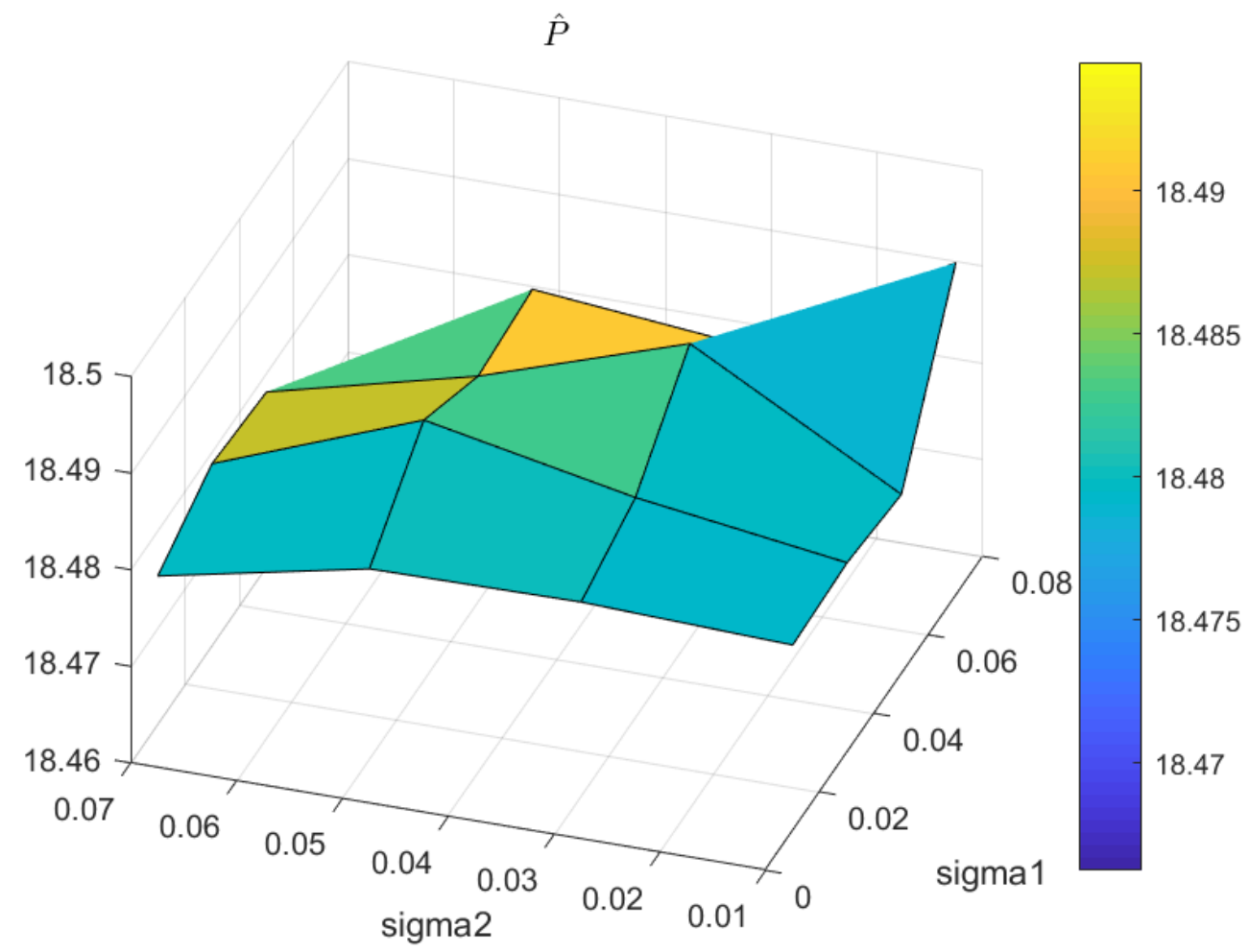

The 3-D graph shows the effect of changing $\sigma_{\text {OntarioGasoline }}$ and $\sigma_{W T I O i l}$ on the price of the barrier option using bivariate Time-Changed model simulation. $\sigma_{\text {WTI Ioil }}$ is $[0.01: 0.02: 0.07]$ and $\sigma_{\text {OntarioGasoline }}$ is $[0.01: 0.02: 0.07]$. 
Figure 4.21: Simulation of Option Price for Ontario Gasoline and WTI Oil in Bivariate Time-Changed Model

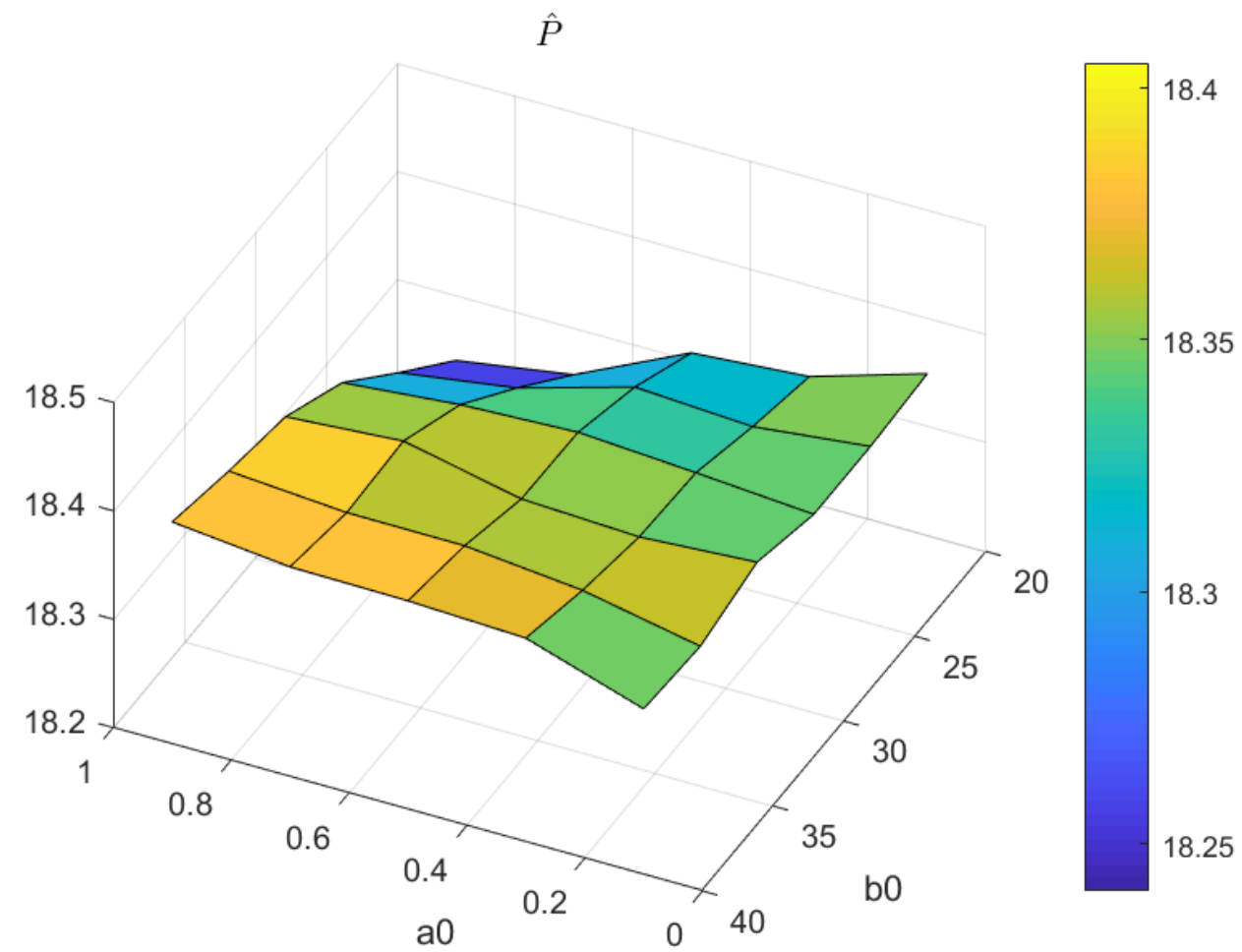

The 3-D graph displays the barrier option price variation with respect to the parameters of $I G\left(a_{0}, b_{0}\right.$ using bivariate Time-Changed model simulation. $a_{0}$ and $b_{0}$ are $[0.1: 0.2: 1]$ and $[20: 2: 40]$ respectably. 
Figure 4.22: Simulation of Option Price for Brent and WTI Oil in Bivariate TimeChanged Model

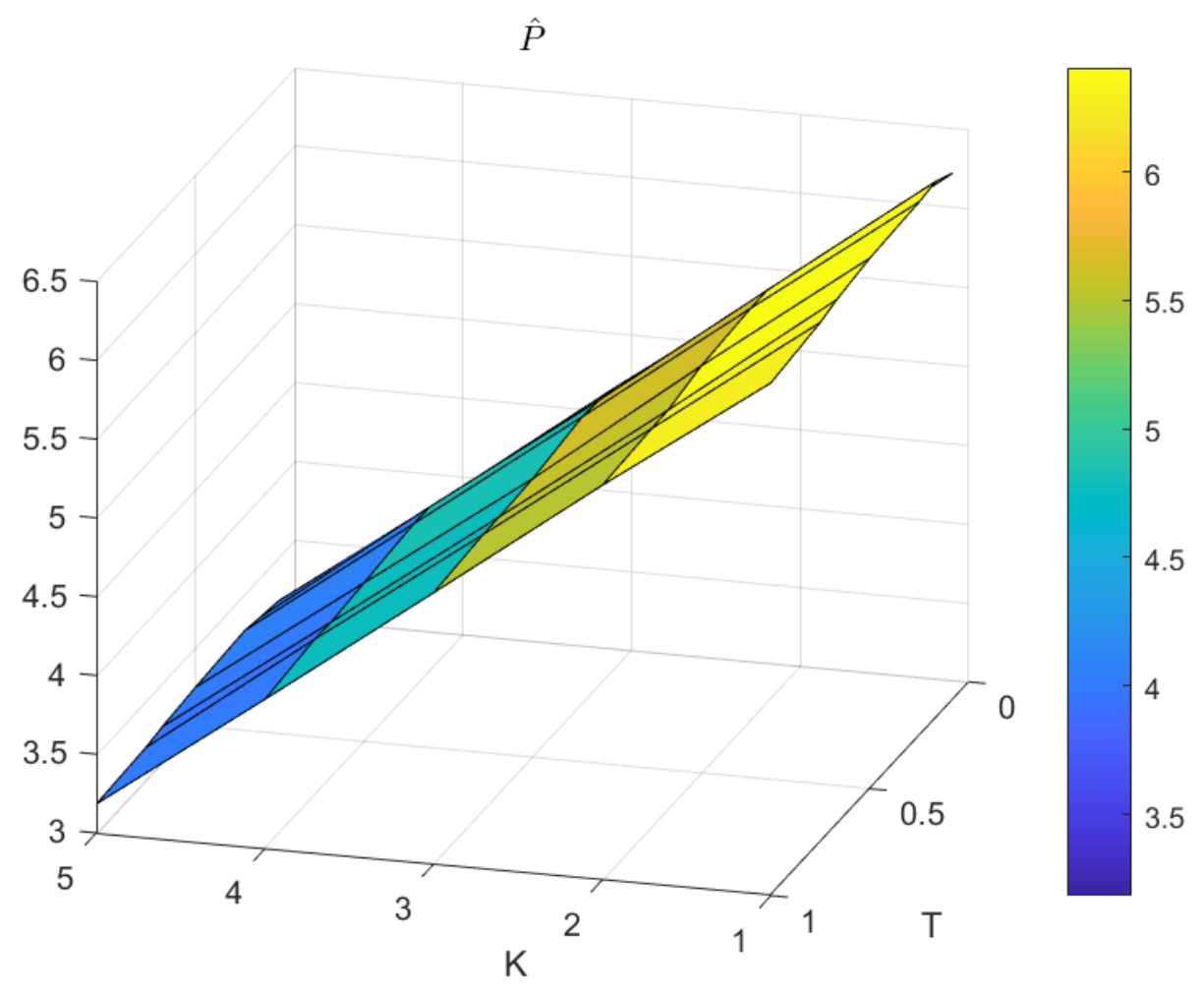

The graph depicts the barrier option price variation with respect to the $\mathrm{T}$ ( maturity of the barrier option), K(Strike price of the barrier option) using bivariate Time-Changed model simulation. The $\mathrm{K}$ is $[1: 1: 5]$ and $\mathrm{T}$ is $\left[\frac{1}{12}: \frac{1}{12}: 1\right]$. 
Figure 4.23: Simulation of Option Price for Brent and WTI Oil in Bivariate TimeChanged Model

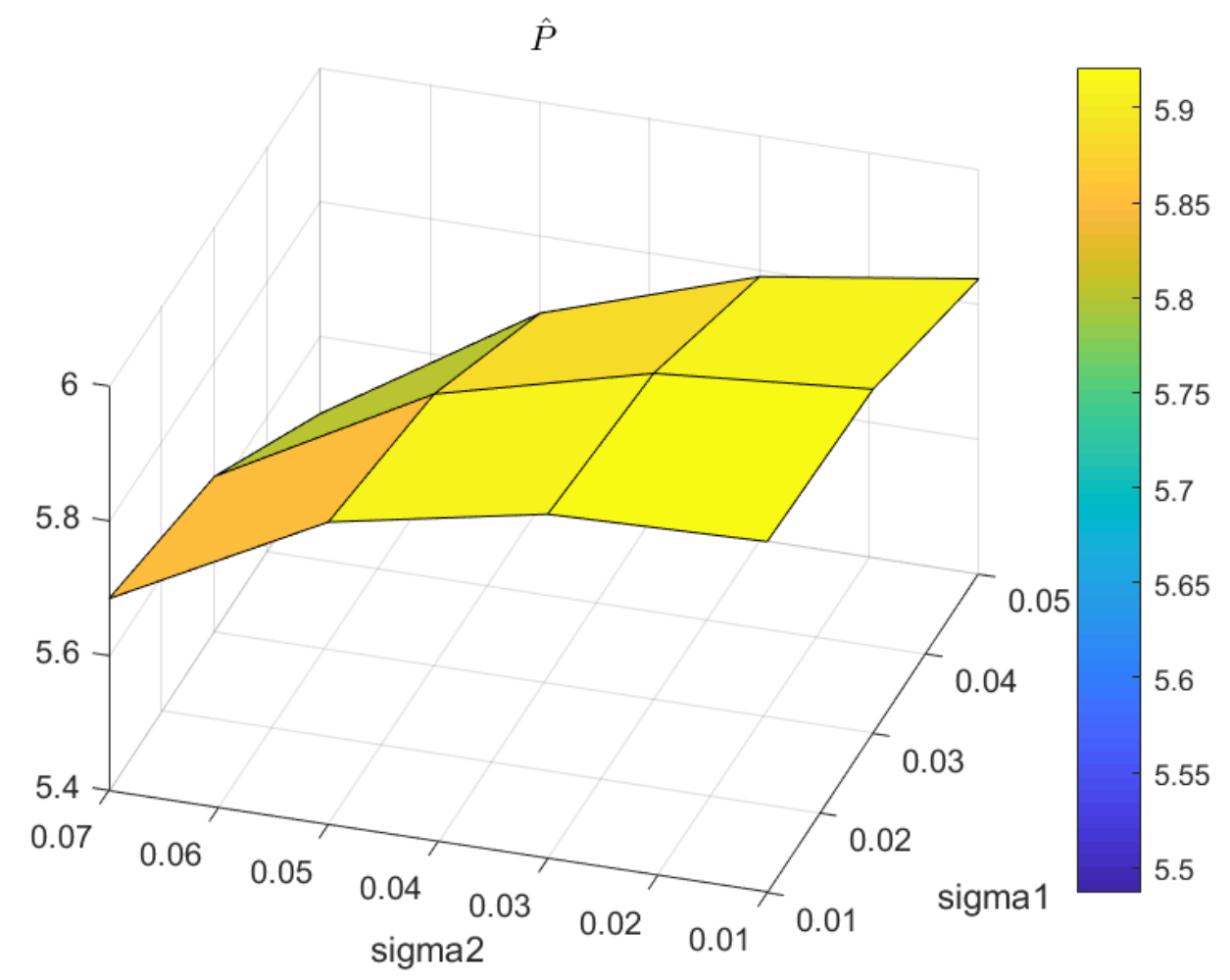

The 3-D graph displays the interplay between $\sigma_{\text {Brentoil }}$ and $\sigma_{W T I o i l}$ and the price of the option using bivariate Time-Changed model simulation. $\sigma_{\text {Brentoil }}$ is $[0.01: 0.02: 0.09]$ and $\sigma_{W T \text { Ioil }}$ is $[0.01: 0.02: 0.1]$ 
Figure 4.24: Simulation of Option Price for Brent oil and WTI Oil in Bivariate Time-Changed Model

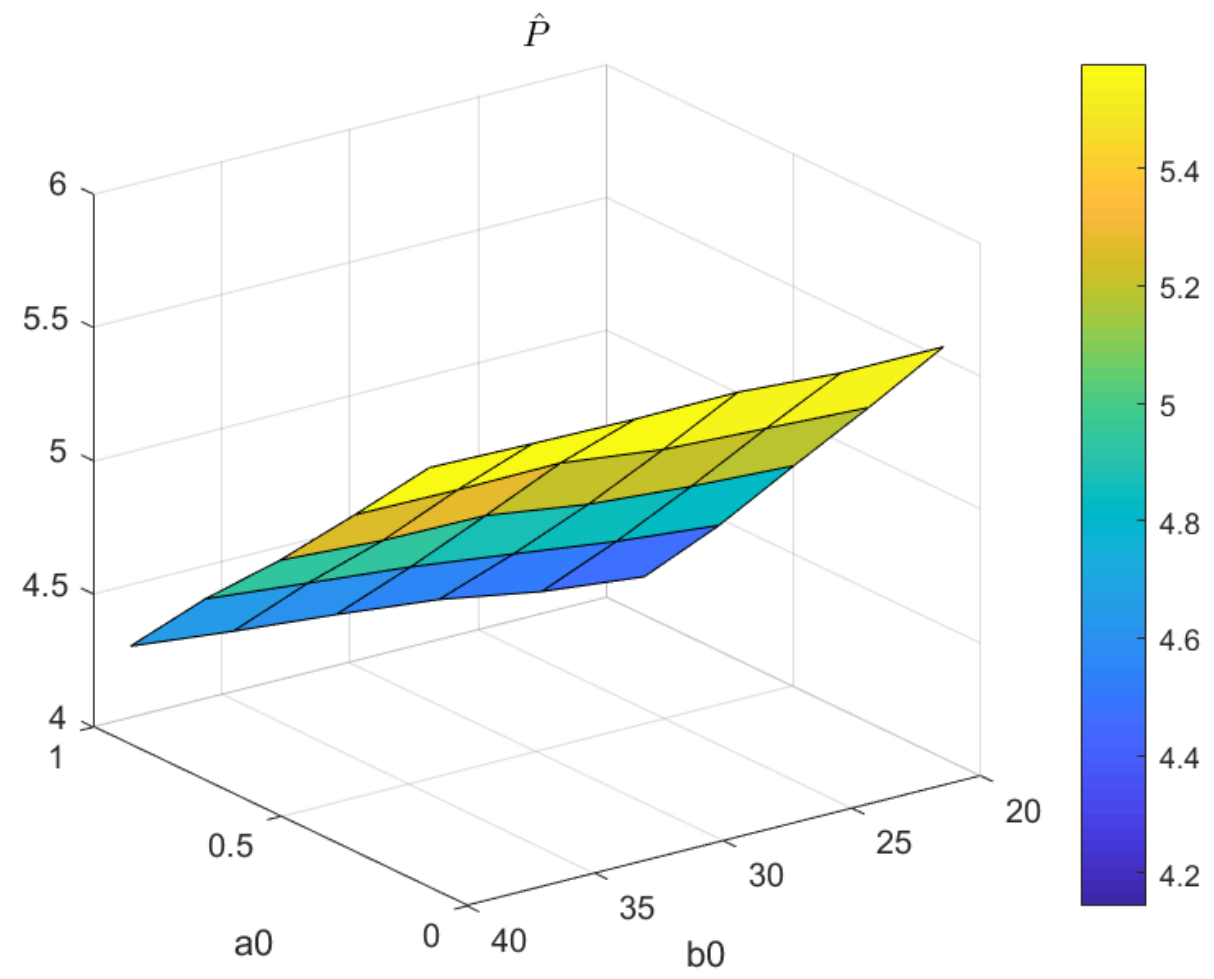

The graph shows barrier option prices change versus te $a_{0}$ and $b_{0}$ that are Inverse

Gaussian parameters based on bivariate Time-Changed model. $a_{0}$ and $b_{0}$ are $[0.1: 0.2: 1]$ and $[20: 2: 40]$ respectably. 


\subsection{Cosine Fast Fourier Transform Method}

Fourier Transform methods provide an efficient and widely-used alternative to MonteCarlo and other numerical methods when the characteristic func- tion of the underlying market model is known. Carr and Madan (Carr and Madan,1999)first used the Fourier transform to price European call options, while both Dempster and Hong (Dempster and Hong, 2000), and Hurd and Zhou. derived Fourier Transform methods to price spread options.

In this section, we used COS Fast Fourier Transform method for barrier option in Time-Changed model.

\subsubsection{COS Fast Fourier Transform Method}

The COS method is imerged from the idea that the Fourier-cosine series coefficients of a density function $f(y \mid x)$ are closely related to its characteristic function.

Since the density function, $f(y \mid x)$, decays to zero rapidly as $y \rightarrow \pm \infty$, we can truncate the infinite integration range in the risk-neutral valuation formula without loosing significant accuracy. Suppose for $[a, b] \subset \mathbb{R}$, we have

$$
\int_{\mathbb{R} \backslash[a, b]} f(y \mid x) d y<T O L
$$

for some given tolerance, TOL, then we can approximate $c\left(x, t_{m-1}\right)$ with replacing the density function by its Fourier-cosine series expansion on $[a, b]$,

$$
f(y \mid x)=\sum{ }_{k=1}^{\infty} A_{k}(x) \cos \left(k \pi \frac{y-a}{b-a}\right)
$$


where $\sum$, indicates that the first term in the summation is multiplied by $\frac{1}{2}$. The series coefficients $\left\{A_{k}(x)\right\}_{k=1}^{\infty}$ are defined by

$$
A_{x}(x):=\frac{2}{b-a} \int_{a}^{b} f(y \mid x) \cos \left(k \pi \frac{y-a}{b-a}\right) d y
$$

Interchanging the summation and integration operators yields

$$
c\left(x, t_{m-1}\right)=\frac{1}{2}(b-a) e^{-r \Delta t} \sum_{k=0}^{\infty} A_{k}(x) V_{k}\left(t_{m}\right)
$$

with $V_{k}\left(t_{m}\right)$ are the Fourier-cosine series coefficients of $v\left(y, t_{m}\right)$ on $[a, b]$,

$$
V_{k}\left(t_{m}\right):=\frac{2}{b-a} \int_{a}^{b} v\left(y, t_{m}\right) \cos \left(k \pi \frac{y-a}{b-a}\right) d y
$$

cutting infinite series gives to $\mathrm{N}$

$$
c^{\prime}\left(x, t_{m-1}\right)=\frac{1}{2}(b-a) e^{-r \Delta t} \sum_{k=0}^{N-1} A_{k}(x) V_{k}\left(t_{m}\right)
$$

conditional characteristic function, $\phi(\omega ; x)$ and $A_{k}(x)$ are related together and can be defined

$$
\phi(\omega ; x):=\int_{\mathbb{R}} f(y \mid x) e^{i \omega y} d y
$$

Consequently, we can write coefficient $A_{k}(x)$ as below

$$
A_{k}(x)=\frac{2}{b-a} \operatorname{Re}\left\{e^{-i k \pi \frac{a}{b-a}} \int_{a}^{b} e^{i \frac{k \pi}{b-a} y} f(y \mid x) d y\right\}
$$

where $R e\{$.$\} is real part of input argument. Finite integration can be approximated$ by following equation

$$
\int_{a}^{b} e^{i \frac{k \pi}{b-a} y} f(y \mid x) d y \approx \int_{\mathbb{R}} e^{i \frac{k \pi}{b-a} y} f(y \mid x) d y=: \phi\left(\frac{k \pi}{b-a} ; x\right) .
$$


Accordingly by $A_{k}(x)$ by $F_{k}(x)$, we have

$$
F_{k}(x):=\frac{2}{b-a} \operatorname{Re}\left\{\phi\left(\frac{k \pi}{b-a} ; x\right) e^{-i k \pi \frac{a}{b-a}}\right\}
$$

after replacing $A_{k}(x)$ in $c^{\prime}$ by $F_{k}(x)$ gives COS formula for exponential Levy processes will be

$$
\hat{c}\left(x, t_{q-1}\right):=e^{-r \Delta t} \sum_{k=0}^{N-1} \operatorname{Re}\left\{\varphi_{\text {levy }}\left(\frac{k \pi}{b-a}\right) e^{i k \pi \frac{x-a}{b-a}}\right\} V_{k}\left(t_{q}\right)
$$

where $\varphi_{\text {levy }}(\omega):=\phi_{\text {levy }}(\omega ; 0)$.

Using $\varphi_{\text {levy }}(\omega):=\phi_{\text {levy }}(\omega ; 0)$ we can calculate $v\left(x, t_{0}\right)$

$$
\hat{v}\left(x, t_{q-1}\right):=e^{-r \Delta t} \sum_{k=0}^{N-1} \operatorname{Re}\left\{\varphi_{\text {levy }}\left(\frac{k \pi}{b-a}\right) e^{i k \pi \frac{x-a}{b-a}}\right\} V_{k}\left(t_{1}\right)
$$

The $V_{j}\left(t_{m}\right), j=0,1, \ldots N-1$ can be recovered from $V_{j}\left(t_{m+1}\right), j=0,1, \ldots N-1$ (Fang and Oosterless).

\subsubsection{Cosine Method in Barrier Option Time Changed}

We consider an exponential univariate Time-Changed Levy model with subordinator, given by a univariate Inverse Gaussian process. The dependence between both underlying assets is given through the Brownian components as well as the TimeChanged subordinator. We also consider a drift given by mean reverting component. As we see in previous section in COS method, we need a characteristic function for the Levy Process. If asset price hits a certain barrier level M at one of observation dates, we call discretely "out" barrier option. This out option be called down-andout option if $M<S_{0}$. In this section we explain COS Method for down-and-out 
option.

The pay off option for down-and-out option reads

$$
h\left(S_{T}\right)=\left(\max \left(\left(S_{T}-K\right), 0\right)\right) \mathbf{1}_{\left\{S_{T}>M\right\}}
$$

The price of down-and-out option that monitored $R$ times, the set of observation dates, $\left\{t_{1}, \ldots, t_{Q}\right\}, t_{1}<\ldots<t_{Q-1}<t_{Q}=T$

$$
\begin{gathered}
\hat{c}\left(S_{t_{q-1}}\right)=e^{-r\left(t_{q}-t_{q-1}\right)} \int_{\mathbb{R}} h\left(S_{t_{q}}\right) f(y \mid x) d y, \\
h\left(S_{t_{q-1}}\right)= \begin{cases}0 & m \geq 0 \\
c\left(S_{t_{q-1}}\right) & x<m .\end{cases}
\end{gathered}
$$

Where $m:=\ln \frac{M}{K}$ and $q=Q, Q-1, \ldots, 2$.

Lemma 4.2. (Backward Induction for Discrete Barrier Option). By backward recursion we find the following numerical approximation for discretely monitored barrier options: For $q=Q-1, Q-2, \ldots, 1$

$$
\hat{V}_{k}\left(t_{q}\right)=\hat{C}_{k}\left(a, m, t_{q}\right)
$$

If $m<0$ we have

$$
V_{k}\left(S_{t_{Q}}\right)=G_{k}(a, m)
$$

For $m \geq 0$,

$$
V_{k}\left(x, t_{Q}\right)=G_{k}(a, 0)
$$


Where

$$
\begin{gathered}
G_{k}\left(x_{1}, x_{2}\right):=\frac{2}{b-a} \int_{x_{1}}^{x_{2}} g\left(x, t_{q}\right) \cos \left(k \pi \frac{x-a}{x-b}\right) d x . \\
g\left(x, t_{q}\right)= \pm K\left(1-e^{x}\right)
\end{gathered}
$$

If $x_{2} \leq 0$ then $g\left(x, t_{q}\right)=K\left(1-e^{x}\right)$, if $x_{1} \geq 0$ then $g\left(x, t_{q}\right)=K\left(e^{x}-1\right)$ and

$$
C_{k}\left(x_{1}, x_{2}, t_{q}\right):=\frac{2}{b-a} \int_{x_{1}}^{x_{2}} c\left(x, t_{q}\right) \cos \left(k \pi \frac{x-a}{x-b}\right) d x
$$

Then we can find the $\hat{v}\left(x, t_{q-1}\right)$ by using (4.9) formula

\subsubsection{COS Method Algorithm}

In this section, we will explain the COS method algorithm for barrier option.We have three parts, initialization, main loop to recover $\left.\hat{V}_{(} t_{q-1}\right)$ and finalization in this algorithm.

\section{Initialization:}

1. In first step we find $V_{k}\left(t_{Q}\right)$ using (4.10) or (4.11).

2. For down-and-out: $x_{1}=m$ and $x_{2}=b$ and $c=a$ and $d=m$.

3. In next step, we construct $\mathbf{m}_{\mathbf{s}}\left(x_{1}, x_{2}\right)$ and $\mathbf{m}_{\mathbf{c}}\left(x_{1}, x_{2}\right)$ by suing following formulas

$$
m_{j}:= \begin{cases}\frac{\left(x_{1}-x_{2}\right) \pi}{b-a} i & \text { if } j=0 \\ \frac{\exp \left(i j \frac{\left(x_{2}-a\right) \pi}{b-a}\right)-\exp \left(i j \frac{\left(x_{1}-a\right) \pi}{b-a}\right)}{j} & \text { if } j \neq 0 .\end{cases}
$$

and

$$
\mathbf{m}_{\mathbf{s}}=\left[m_{0}, m_{-1}, m_{-2}, \ldots, m_{1-N}, 0, m_{N-1}, m_{N-2}, \ldots, m_{1}\right]^{T}
$$


and

$$
\mathbf{m}_{\mathbf{c}}=\left[m_{2 N-1}, m_{2 N-2}, \ldots, m_{2}, m_{1}, m_{0}\right]^{T} .
$$

4. Take $\left.d_{1}=D\left\{\mathbf{m}_{\mathbf{s}} x_{1}, x_{2}\right)\right\}, d_{2}=\mathbf{s g n} . D\left\{\mathbf{m}_{\mathbf{c}}\left(x_{1}, x_{2}\right)\right\}$. Where $\mathbf{s g n}=[1,-1,1,-1, \ldots]^{T}$.

Main Loop to Recover $\hat{V}\left(t_{q-1}\right)$ : For $q=Q$ to 2

1. Computing $\mathbf{u}\left(t_{m}\right)$ that giving by formula:

$$
\mathbf{u}:=\left\{u_{j}\right\}_{j=0}^{N-1}, \quad u_{j}=\varphi_{\text {levy }}\left(\frac{j \pi}{b-a}\right) V j\left(t_{m+1}\right), \quad u_{0}=\frac{1}{2} \varphi_{\text {levy }}(0) V_{0}\left(t_{m+1}\right)
$$

2. constructing $\mathbf{u}_{\mathbf{s}}$ by adding $N$ zeros to $\mathbf{u}\left(t_{m}\right)$.

3. Put $\mathbf{M s u}=$ the first $2 N$ elements of $D^{-1}\left\{d_{1} . D\left(\mathbf{u}_{\mathbf{s}}\right)\right\}$.

4. Take $\mathbf{M c u}=$ reverse $\left\{\right.$ the firstNelemntsof $\left.D^{-1}\left\{d_{2} . D\left(\mathbf{u}_{\mathbf{s}}\right)\right\}\right\}$.

5.

$$
\hat{C}\left(t_{q-1}\right)=\frac{e^{-r \Delta t}}{\pi} \operatorname{Im}\{\mathbf{M s u}+\mathbf{M} \mathbf{c u}\}
$$

6. In the last step of this part of algorithm is enough to take $\hat{V}\left(t_{m-1}\right)=\hat{C}\left(t_{m-1}\right)$.

\section{Finalization:}

Computing $\hat{v}\left(x, t_{q-1}\right)$ by using (4.9). 


\section{Chapter 5}

\section{CONCLUSION}

In this thesis we have studied barrier option pricing using the conventional wellknown but irrealistic Black-Scholes model and its counterpart advanced Time-Changed model. Two scenarios; single asset or univariate and two assets or bivariate option price with utilizing real price of Oil and Gas are analyzed. As expected, the advanced Time-Changed model could provide much different estimation for barrier option price than the Black-Scholes model over the same data.

In univariate barrier option pricing scenario, we could use Brent and WTI oil and Ontario Gasoline real data and prove that Time-Changed model barrier option price give much closer estimation (higher price) than univariate Black-Scholes model (lower price).

In two asset barrier option, one underlying asset, $S_{1}$, determined how much the option is out of money and the other asset, $S_{2}$, is linked to barrier hit in BlackScholes model.(Haug)Because of the barrier has not been taken on the difference price for two underlying assets, therefore, the bivariate barrier option price is not very genuine. On the other hand, the barrier and strike price are came both from the difference price for underlying assets in Time-Changed model that makes the model more realistic than the bivariate Black-Scholes model.For future works, find- 
ing the formula for bivariate Black-Scholes model when the barrier and strike price are based on the difference price of underlying assets, is a good idea.

To obtain the volatilities and drifts for all underlying assets, the data should be matched by same date for both underlying assets, as daily price was available for Brent and WTI oil and weekly price was available for Ontario Gasoline. Manual alignment of data was performed to obtain accurate empirical volatilities and drift value for Brent and WTI to use them in bivariate Time-Changed model.

The Monte-Carlo method is used for simulating the barrier price of both univariate and bivariate Time-Changed model with one million times repetition. consequently, each simulation could take couple of days to completed which can be very challenging and expensive in fast changing real markets. Accordingly, new promising Fast Fourier Cosine technique was introduced where its implementation was presented in the last section of the thesis for further studies. This Fast Fourier Transform technique could be much faster than Monte-Carlo method. 


\section{REFERENCES}

1) Boxma, O. J., Yechiali, U. (2008). Poisson Processes. Encyclopaedia of Statistics in Quality and Reliability.

2) Carmona, R and Durrleman, V. (2003). Pricing and Hedging Spread Options. SIAM Review. 45:4 627-685.

3) Carr, P., Madan, D. (1999). Option valuation using the fast Fourier transform, Journal of Computational Finance, 2(4), 61-73.

4) Carr. P., Liuren,W. (2003). Time-Changed Levy Processes and Option Pricing. Journal of Financial Economics.

5) Cont, Rama and Tankov, P.(2004). Financial Modelling with Jump Processes. Boca Raton, FL: Chapman and Hall CRC.

6) Dahl. Carol A., (2015). International Energy Markets , 2nd edition, Pennwell

7) Dempster, M.A.H. and Hong,S.S.G, (2000). Spread option valuation and the fast Fourier transform. Mathematical Finance - Bachelier Congress, 203-220, Springer.

8) Edwards, D.W, (2010). Energy Trading \& Investing. Trading, Risk managment, and Structuring Energy Markets. 
9) Fang, F. and Oosterlee, C.W. A novel option pricing method based on FourierCosine series expansions. SIAM J.Scri. Comput. 31(2), 826-848

10) Fang, F. and Oosterlee, C.W., (2009). Pricing Early-Exercise and Discrete Barrier Options by Fourier-Cosine Series Expansions.

11) Hazewinkel, M. ed. (2001), Law of large numbers, Encyclopedia of Mathematics, Springer, ISBN 978-1-55608-010-4

13) Hauge, E. G., The Complete Guid to Option Pricing Formula,. Second edition, McGrow-Hill.

14) Heynen, R.C. and Kat, A. M. (1994). Crossing Barriers, Risk Magazine,7.

15) Heynen, R.C. and Kat, A. M. (1994). Partial Barrier Options, Journal of Financial Engineering,3, 253-274.Crossing Barriers, Risk Magazine,7.

16) Hull.J.C., Options, Future and Other Derivatives, nine edition( 2015).

17) Hurd, T.R. and Zhuowei, Z., (2010). A Fourier Transform Method for Spread Option Pricing. SIAM J. Finan. Math., 1, 142-157.

18) James, Tom, (2003). Energy Price Risk, Palgrave Macmillan.

19) James, T., (2003). Energy Market Price Risk Managment and Tradin,Wiley Finance.

20)Kienitz.J, Wetterrau, D.(2012). Financial Modeling, Theory, Implementation and practice, Wiley\& Son. 
21) Marie Mack, Iris, (2014). Energy Trading and Risk managment", Wiley \& Sons.

22) Merton, R. C. (1973). Theory of Rational Option Pricing, Bell of journal of Economics and Managment science,4, 141-183.

23) Michael, J. R., Schucany, W. R. AND Haas, R. W. (1976). Generating Random Variates Using Transformations with Multiple Roots. The American Statistician, 30(2), 88 .

24) Olivares, P., (2018). Valuing Path Dependent Cracks And Quanto Spreads. Working Paper, 2018

25) Papapantoleon, A, (2000). An Introduction to Levy Processes with Applications in Finance.

26)Rich, D. R.(1994). The Mathematical foundation of Barrier Option Pricing Theory, Advance in Future and Optionns research, 7, 267-311.

27) Rubinsten, M.(1990). Double Truble Risk Magazine, 5.

28) Sandri, M. (1996). Numerical Calculation of Lyapunov Exponents. Mathematica J. 6, 78-84.

29) Schoutens, W. (2003). Levy process in finance: Pricing financial derivatives. Chichester, West Sussex: J. Wiley. 
30) Yuri Prokhorov. Strong law of large numbers. Encyclopedia of Mathematics.

117

31) Zhang. P.G., Exotic Options, second edition (1998). 


\section{.1 MATLAB Codes}

\section{Appendix}

MATLAB Codes

Pricing Barrier Option

\section{A. Black-Scholes Model}

\section{A.1 Univariate Black-Scholes Model}

\section{Vanilla Barrier Option}

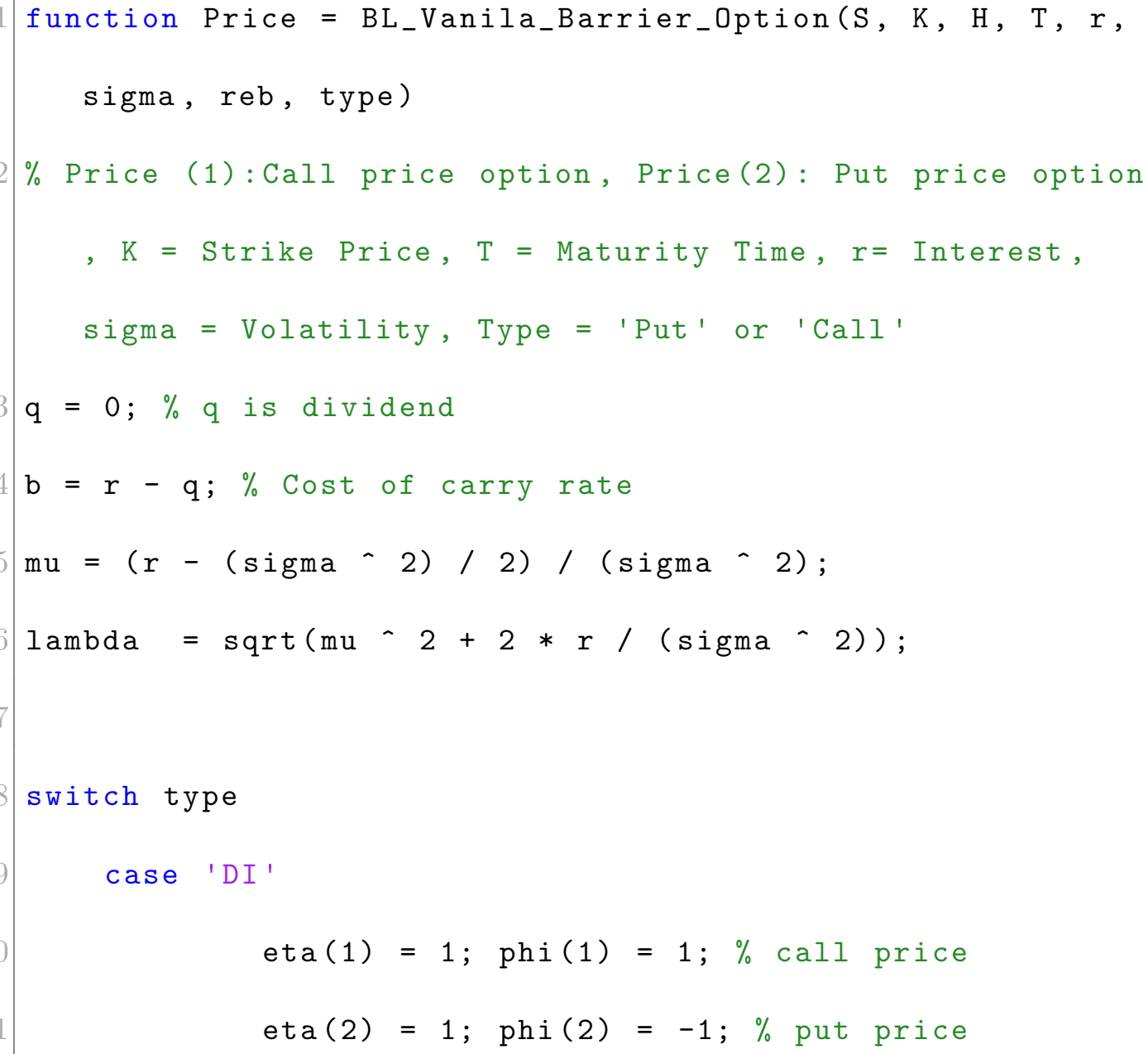




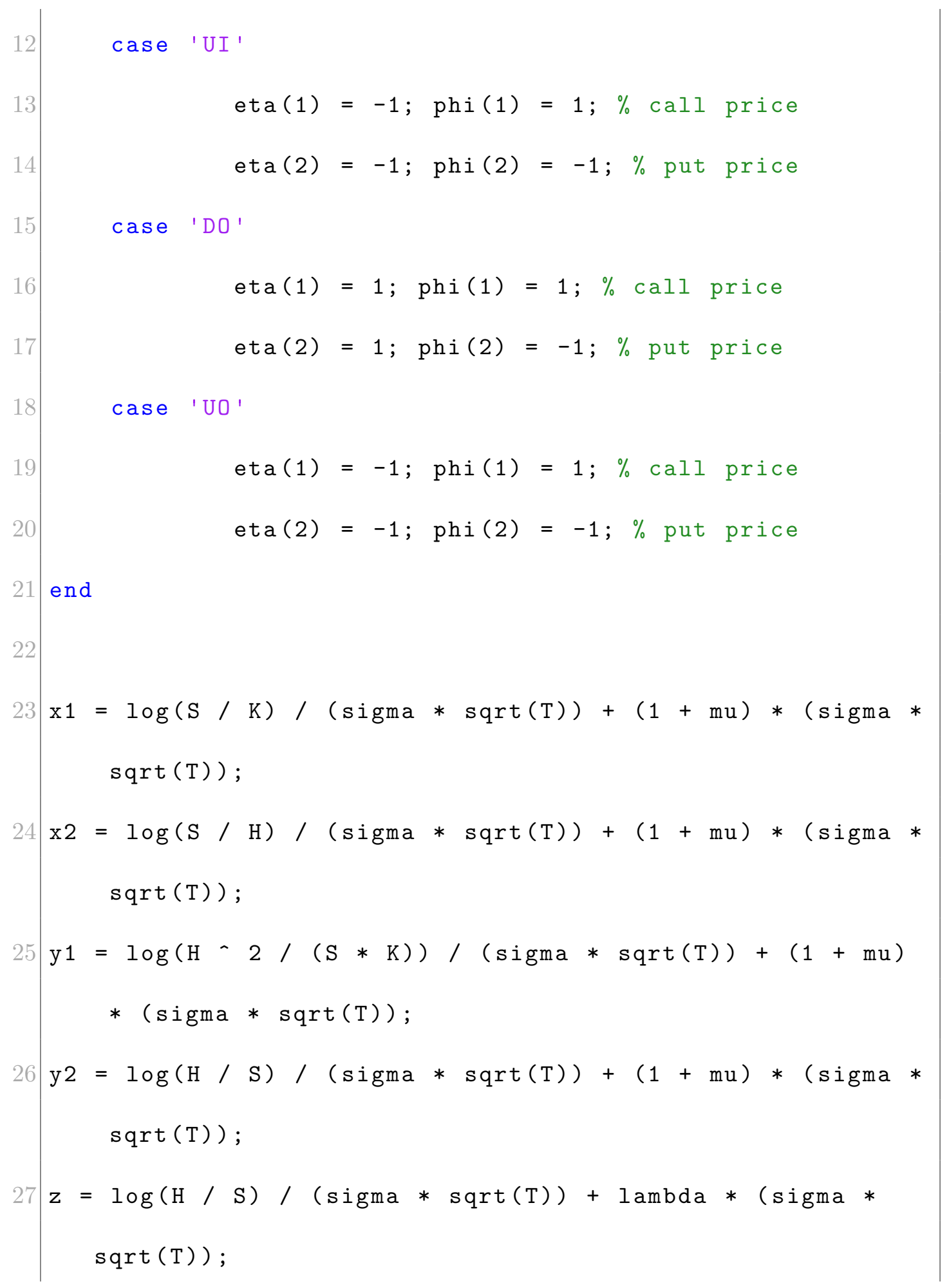




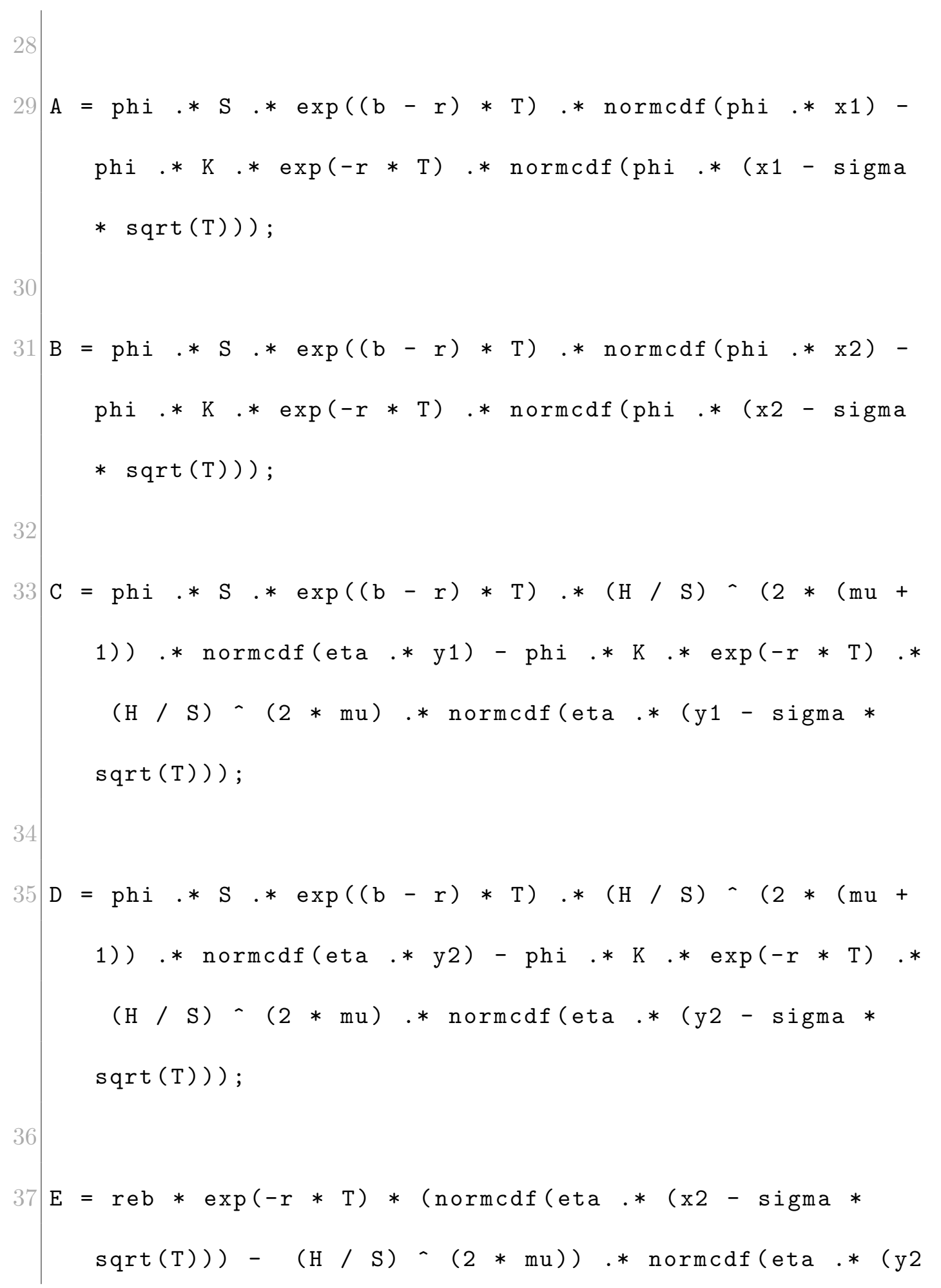




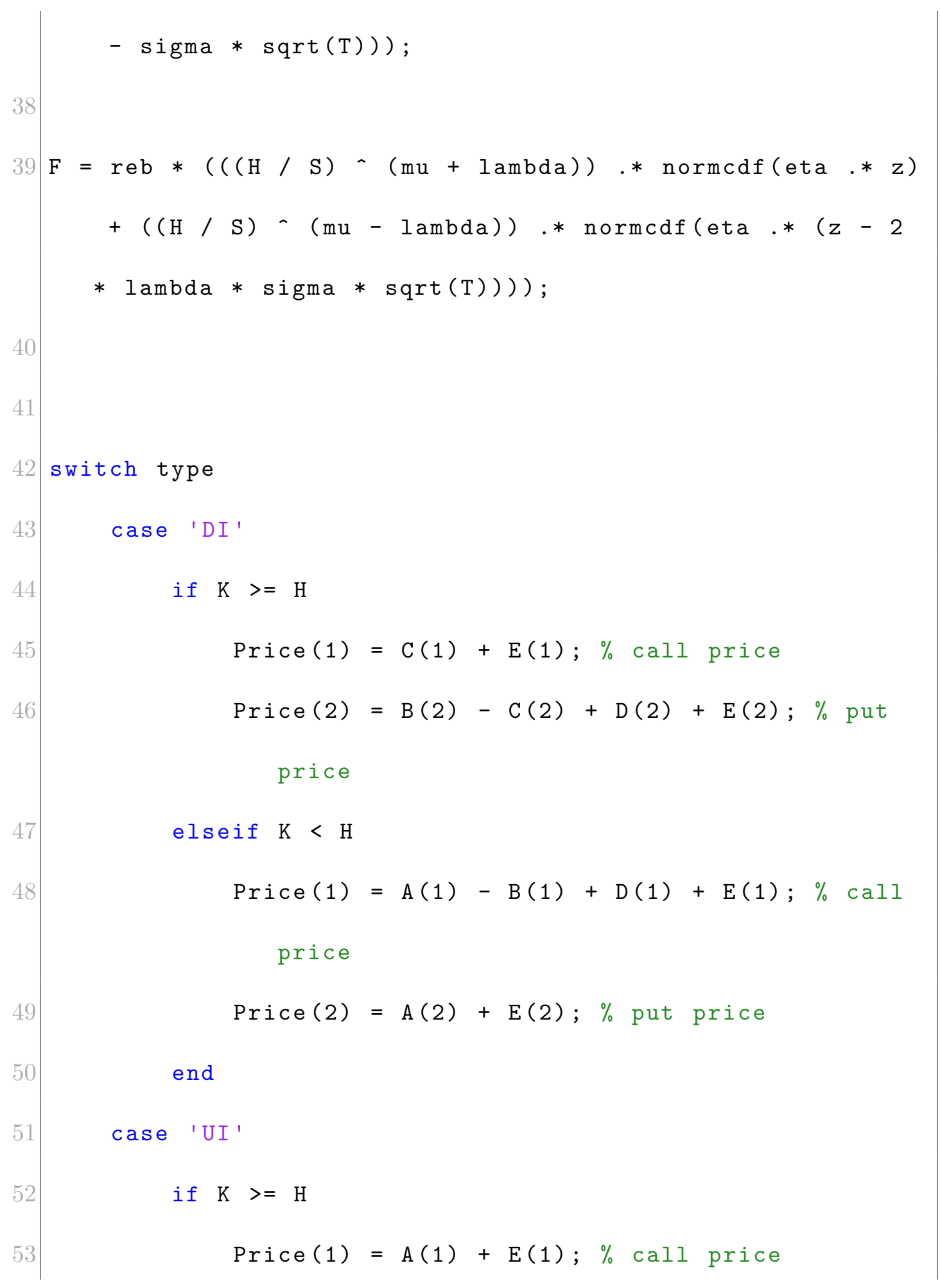




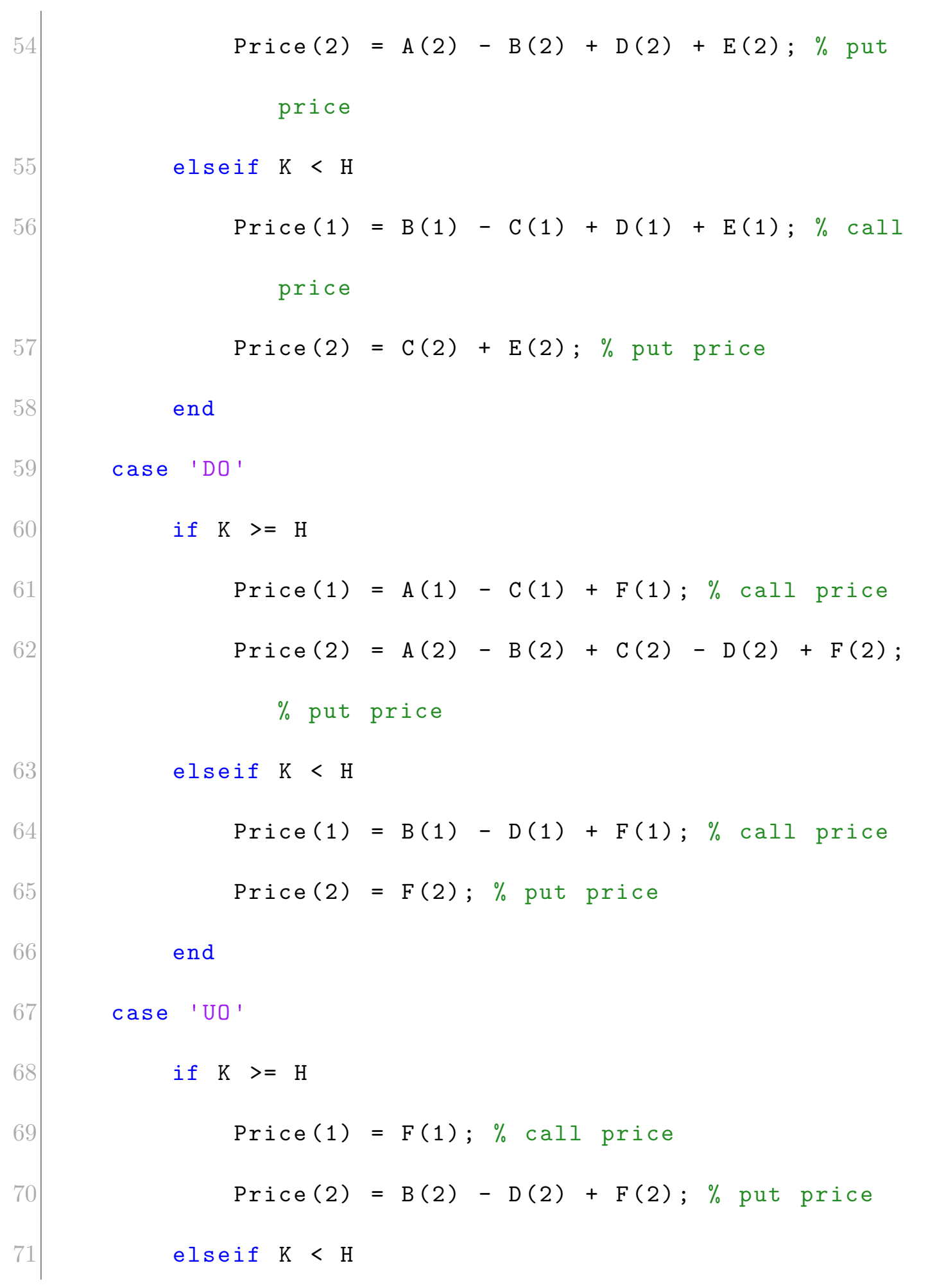




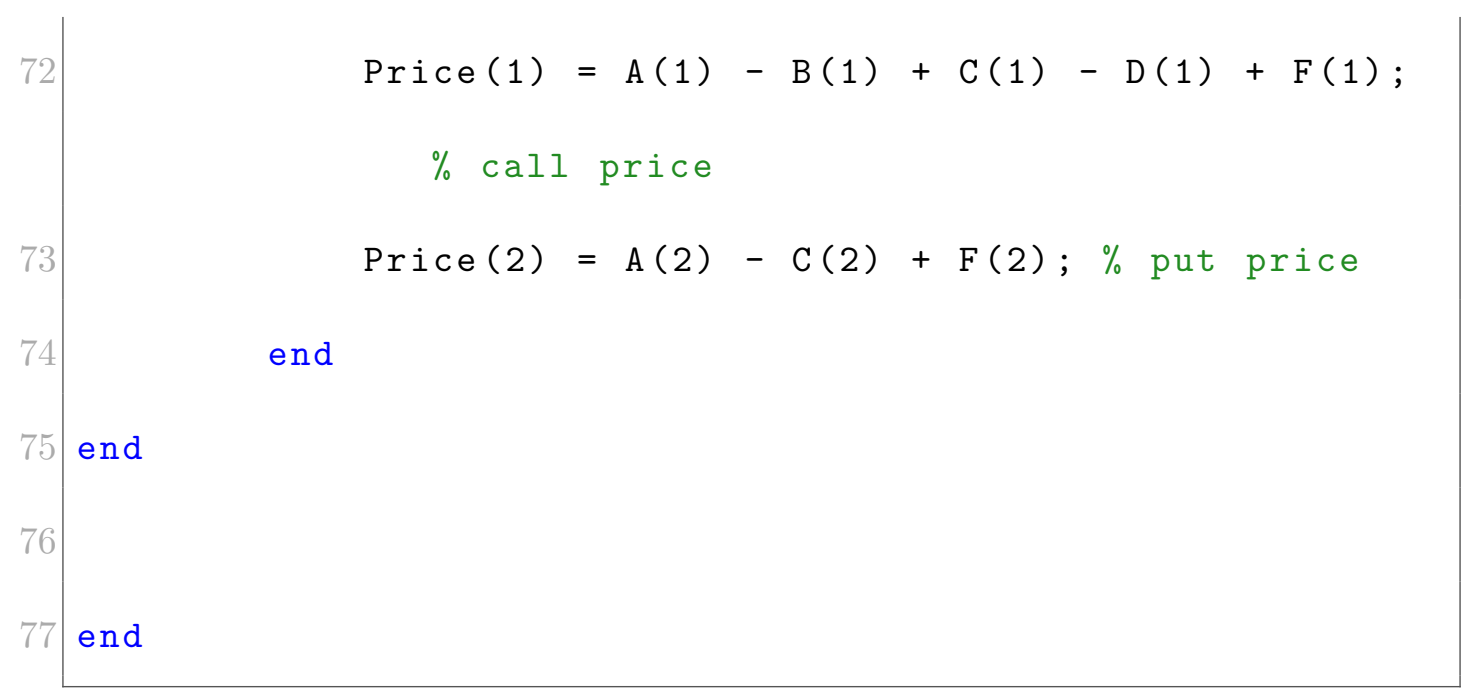

Mainscript Univariate Black-Scholes Model

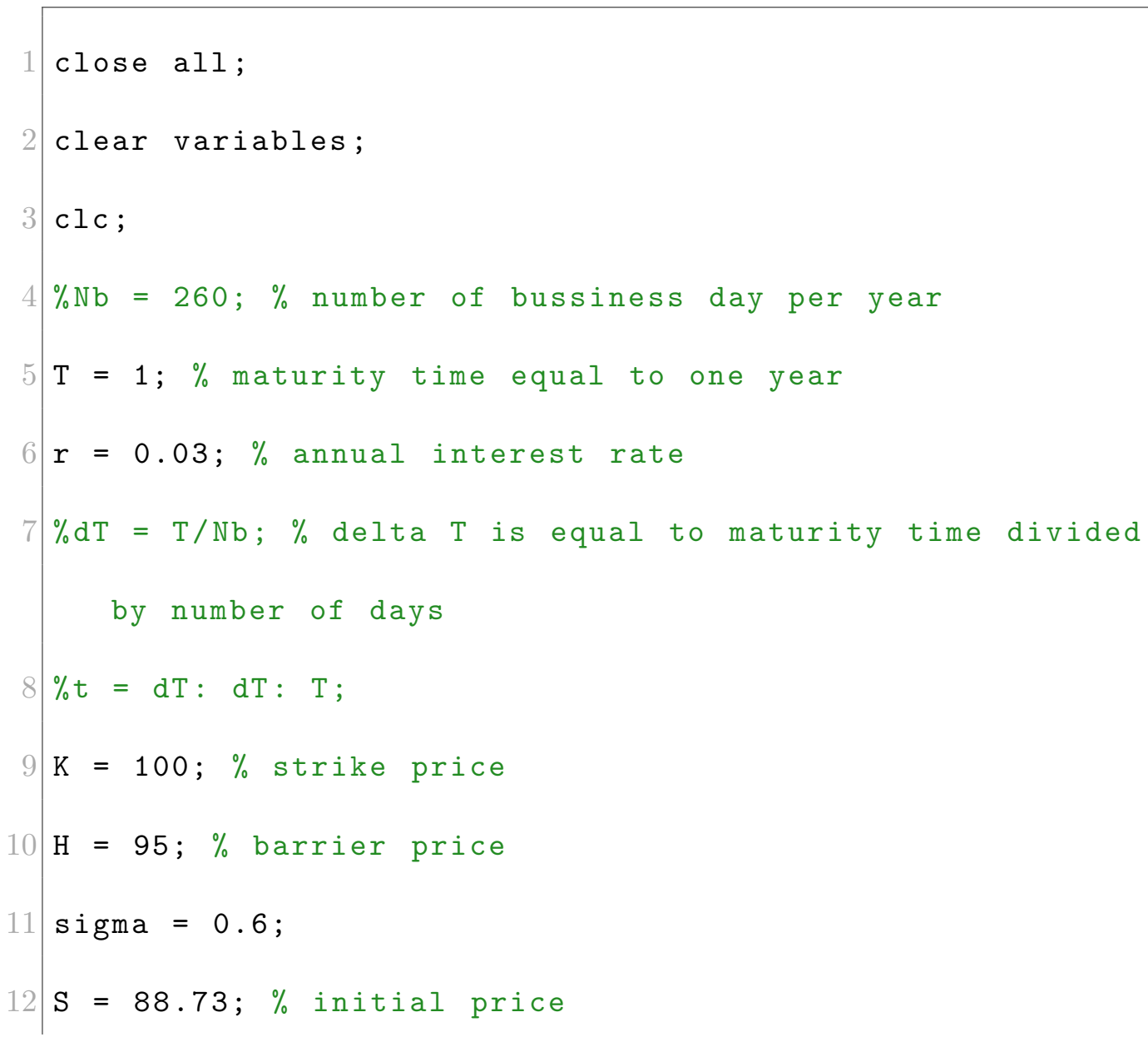




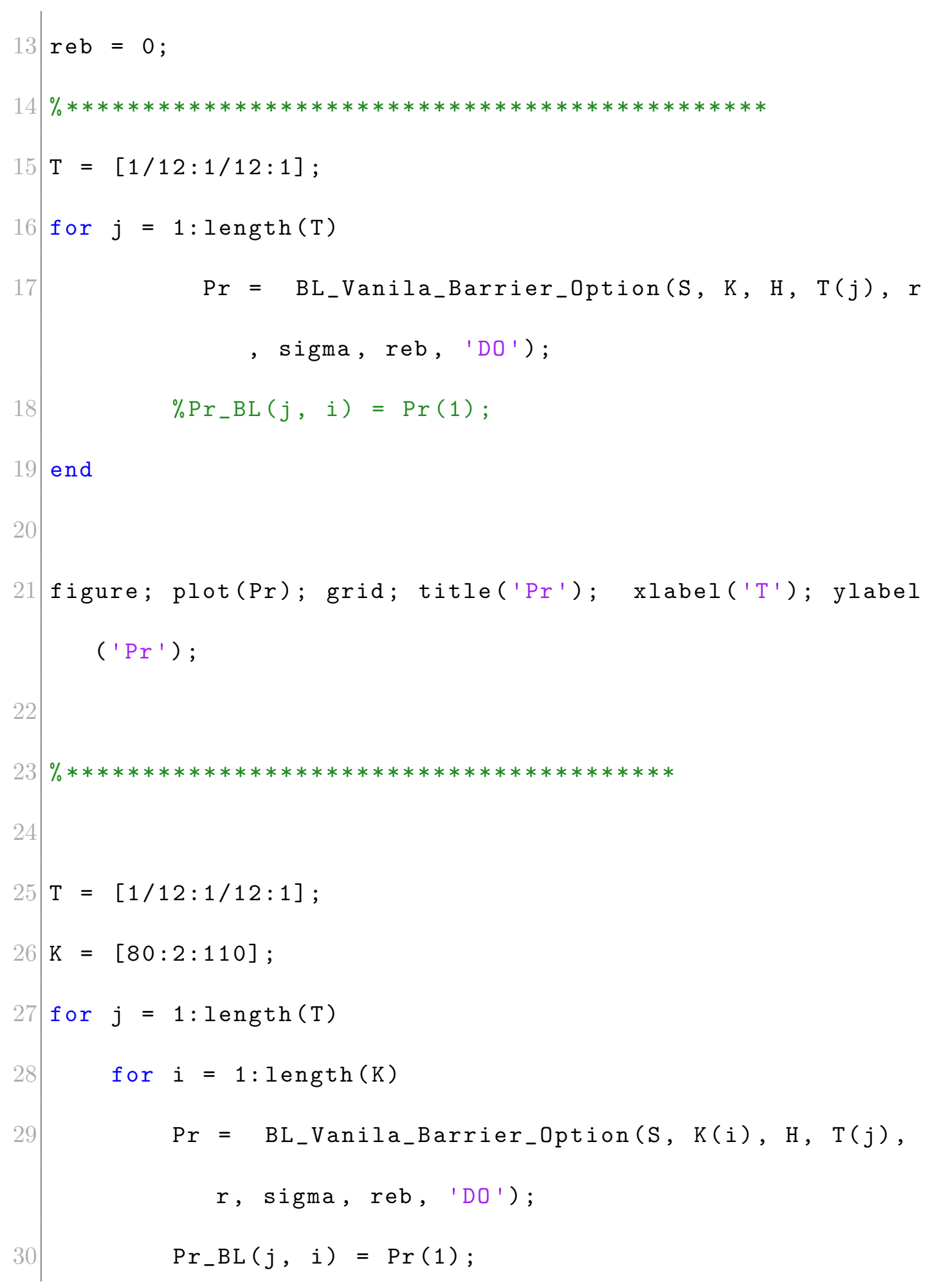




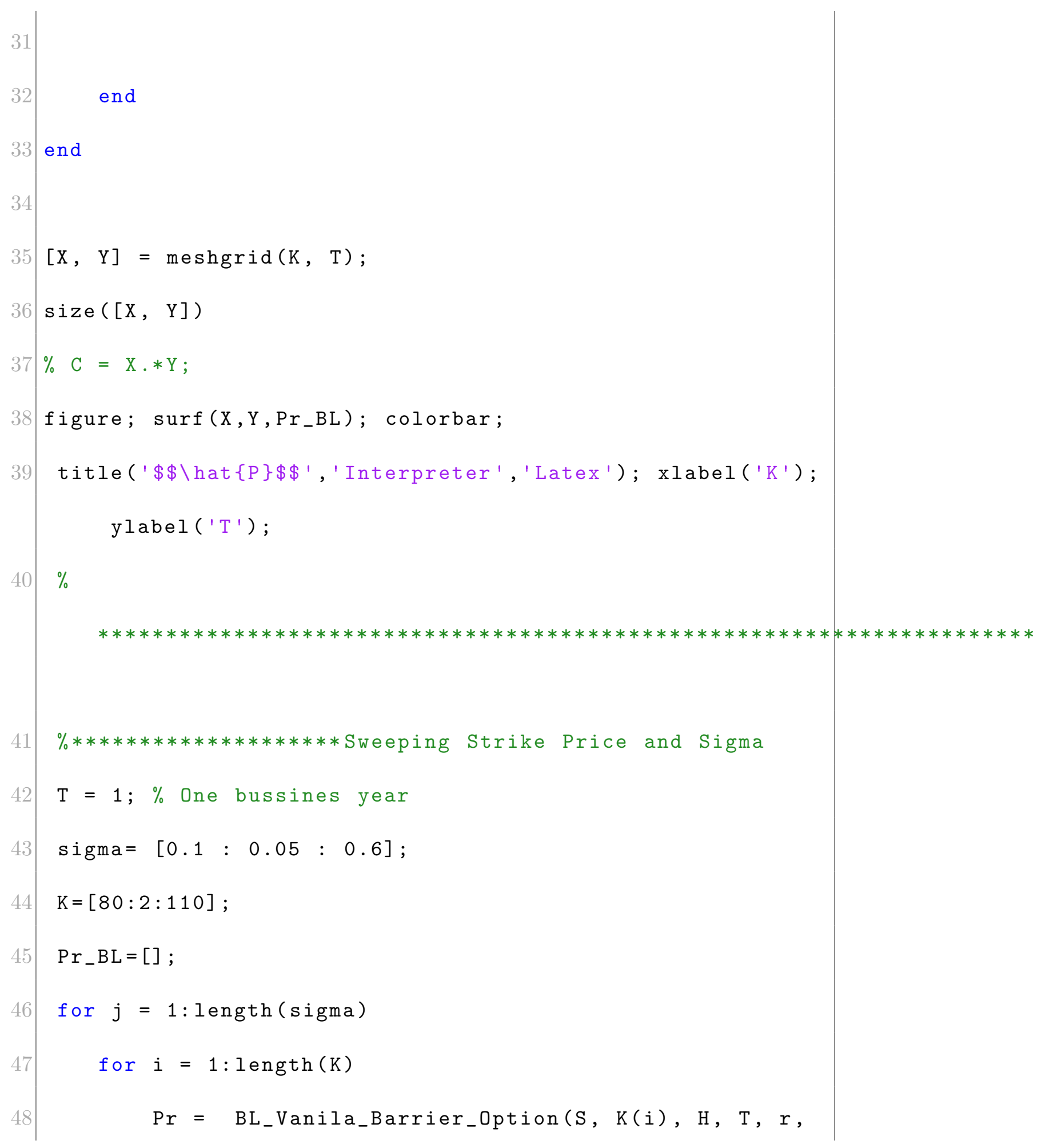




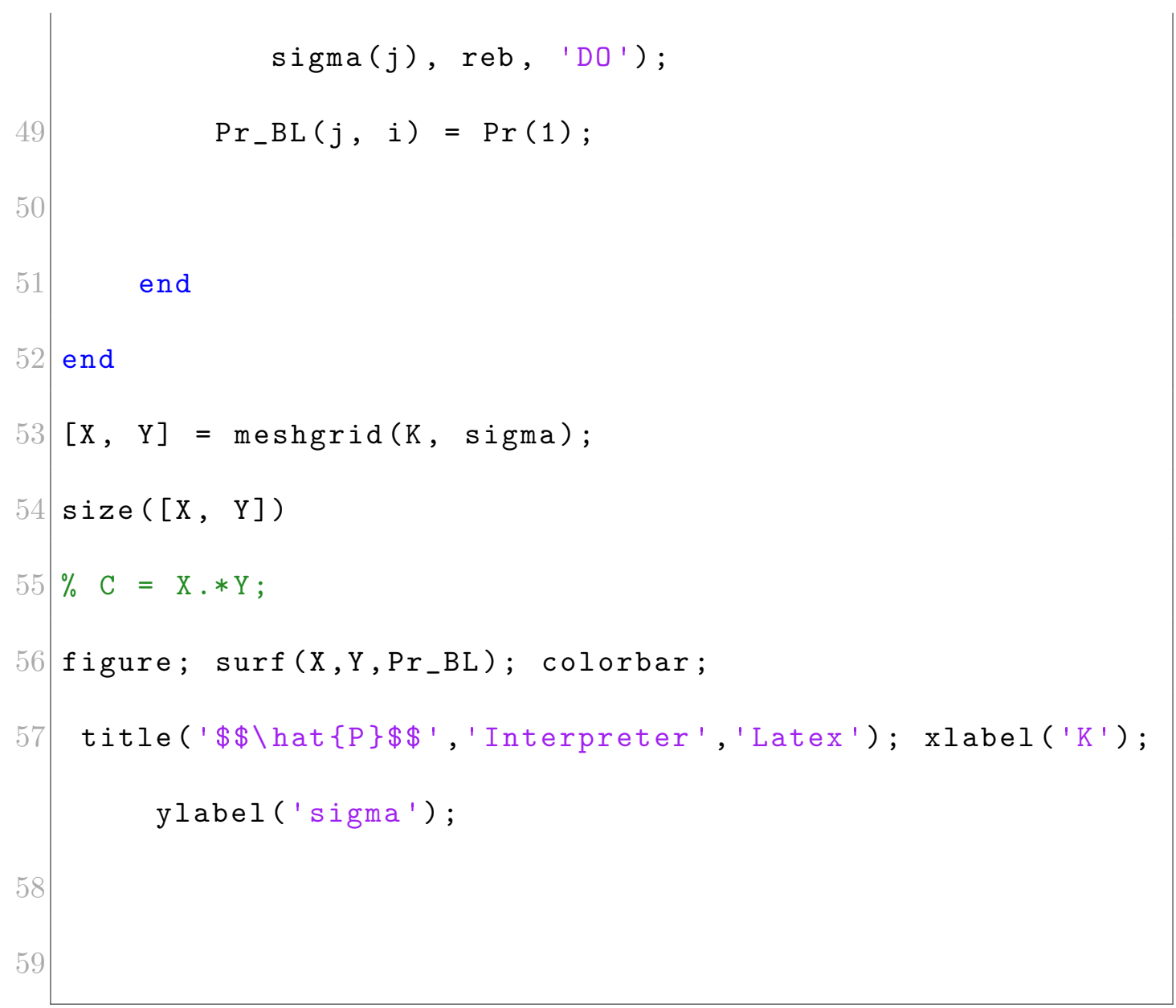

\section{A.2 Bivariate Black-Scholes Model Arcsin}

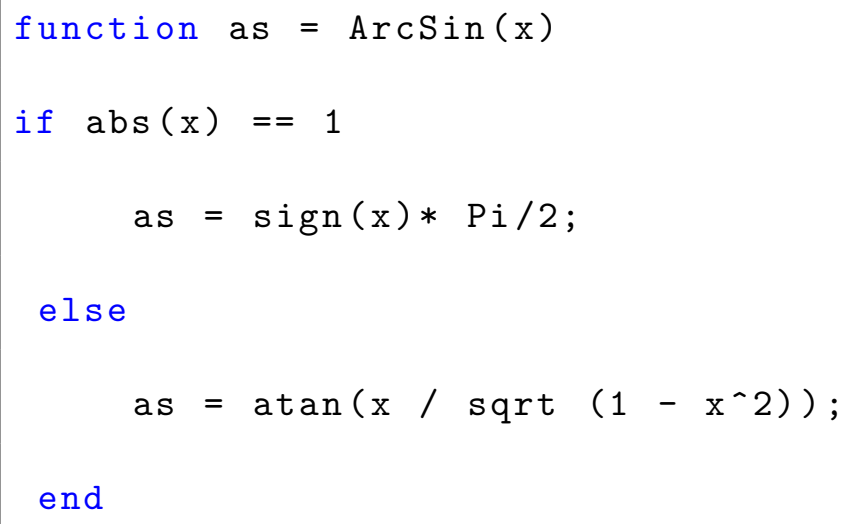




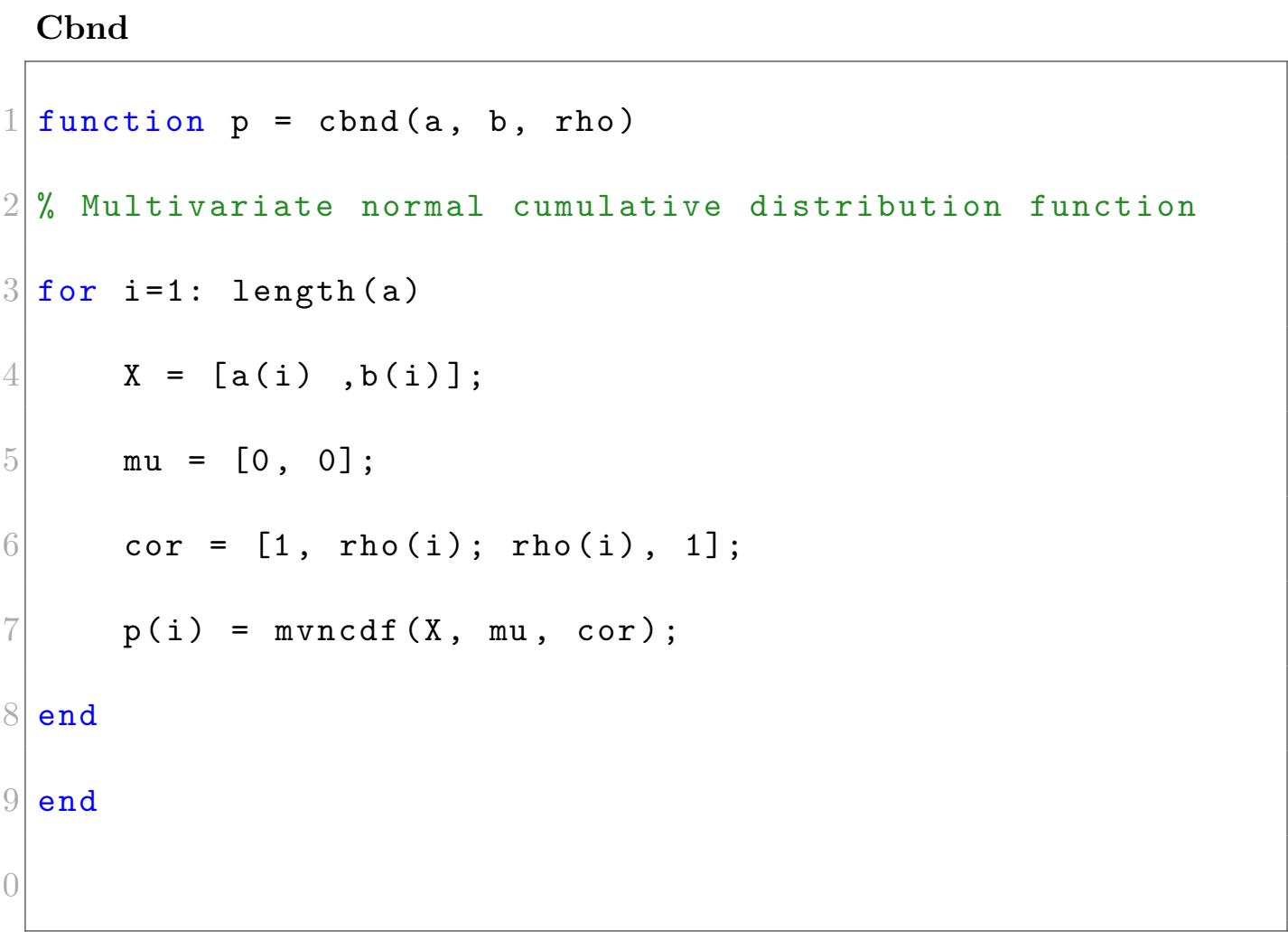

\section{Bivariate Vanilla Barrier Option}

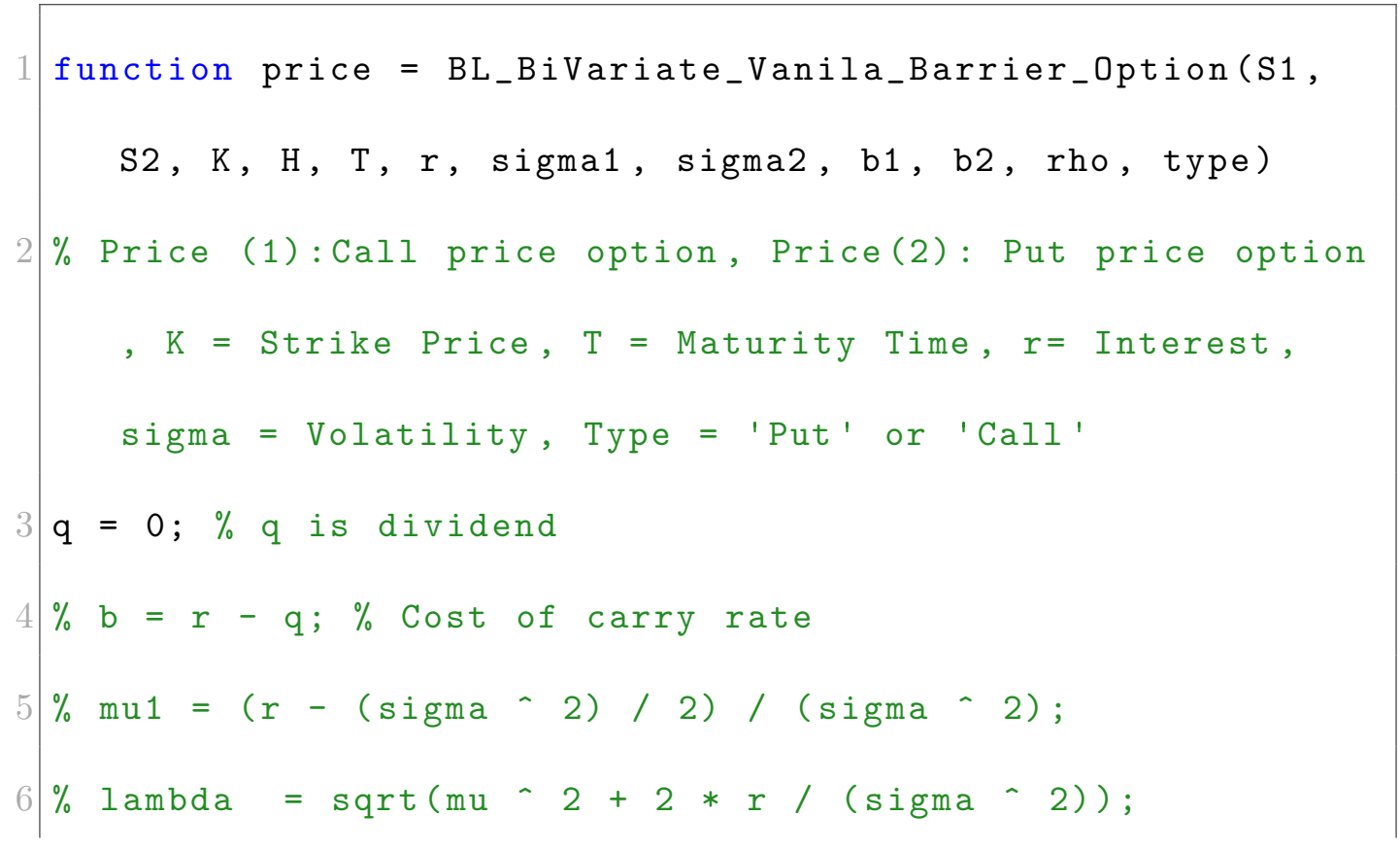




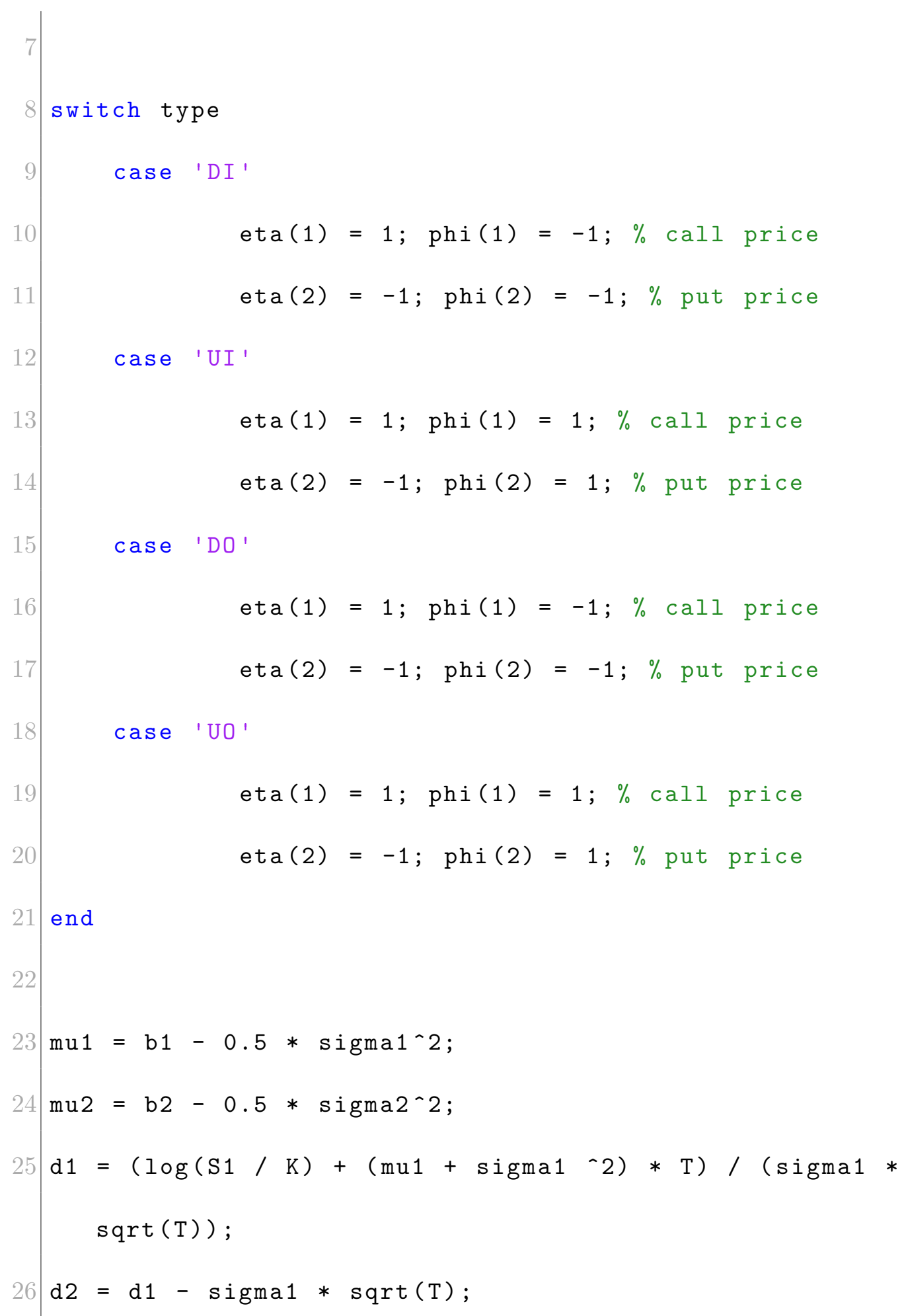




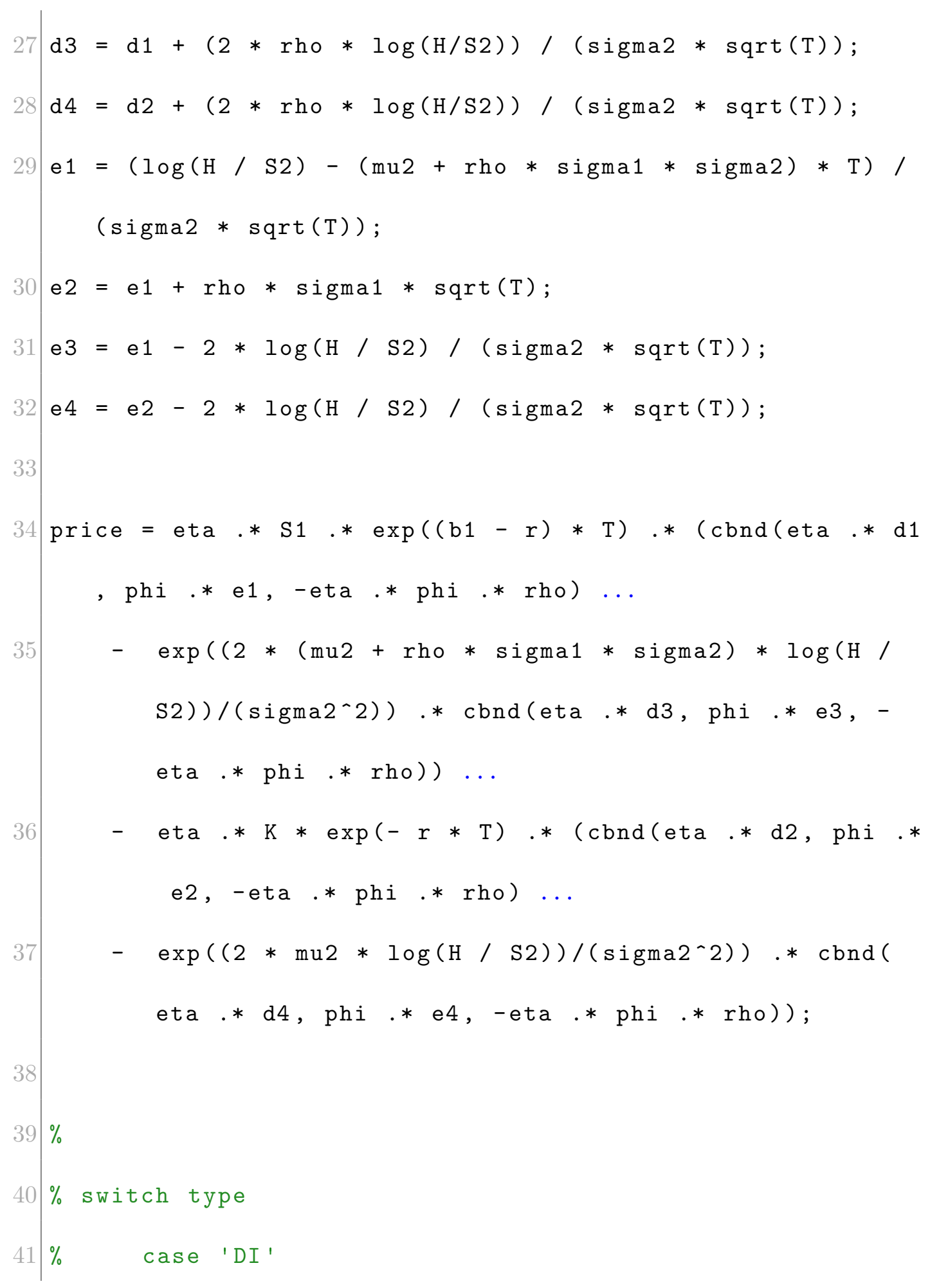




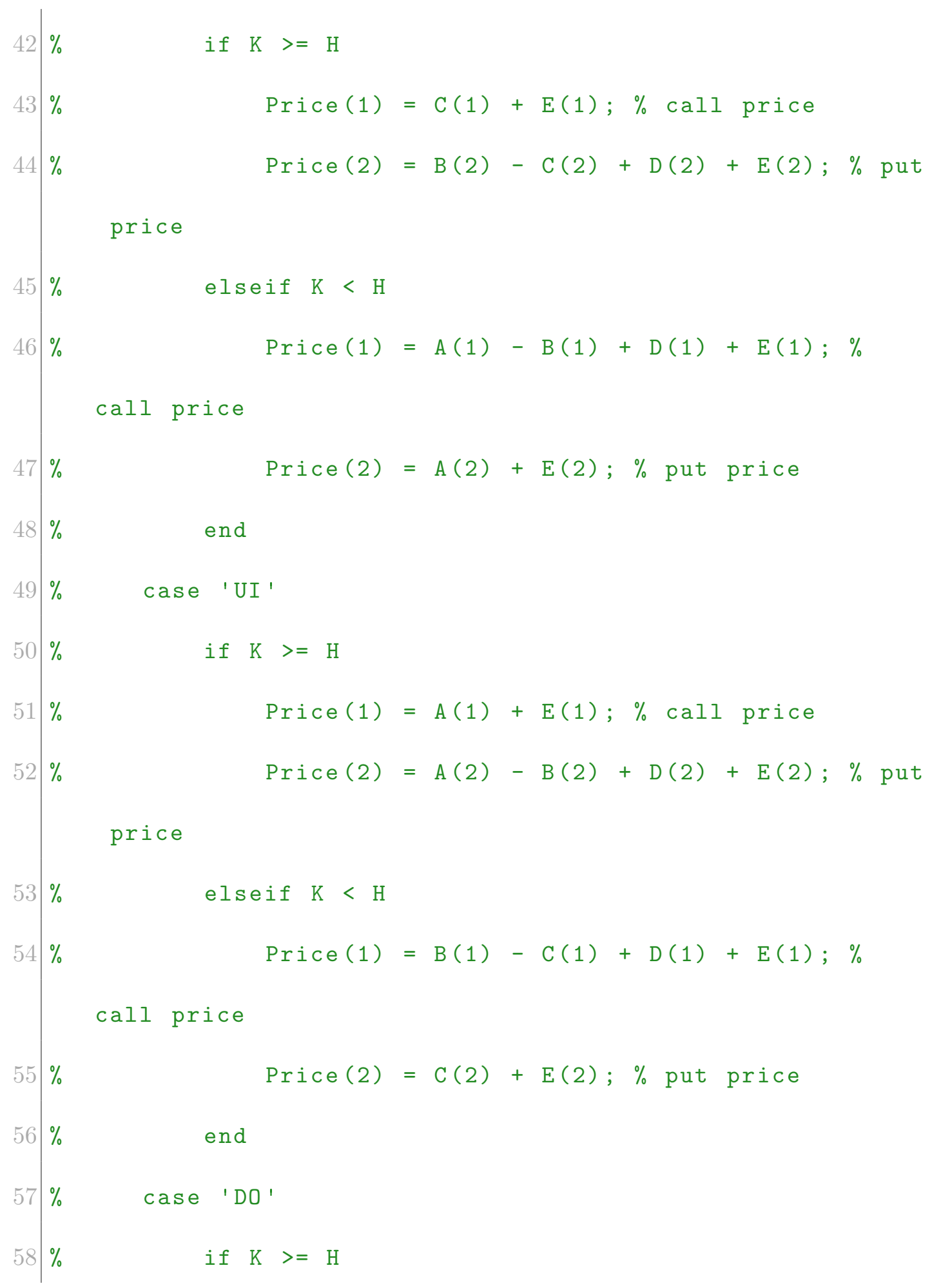




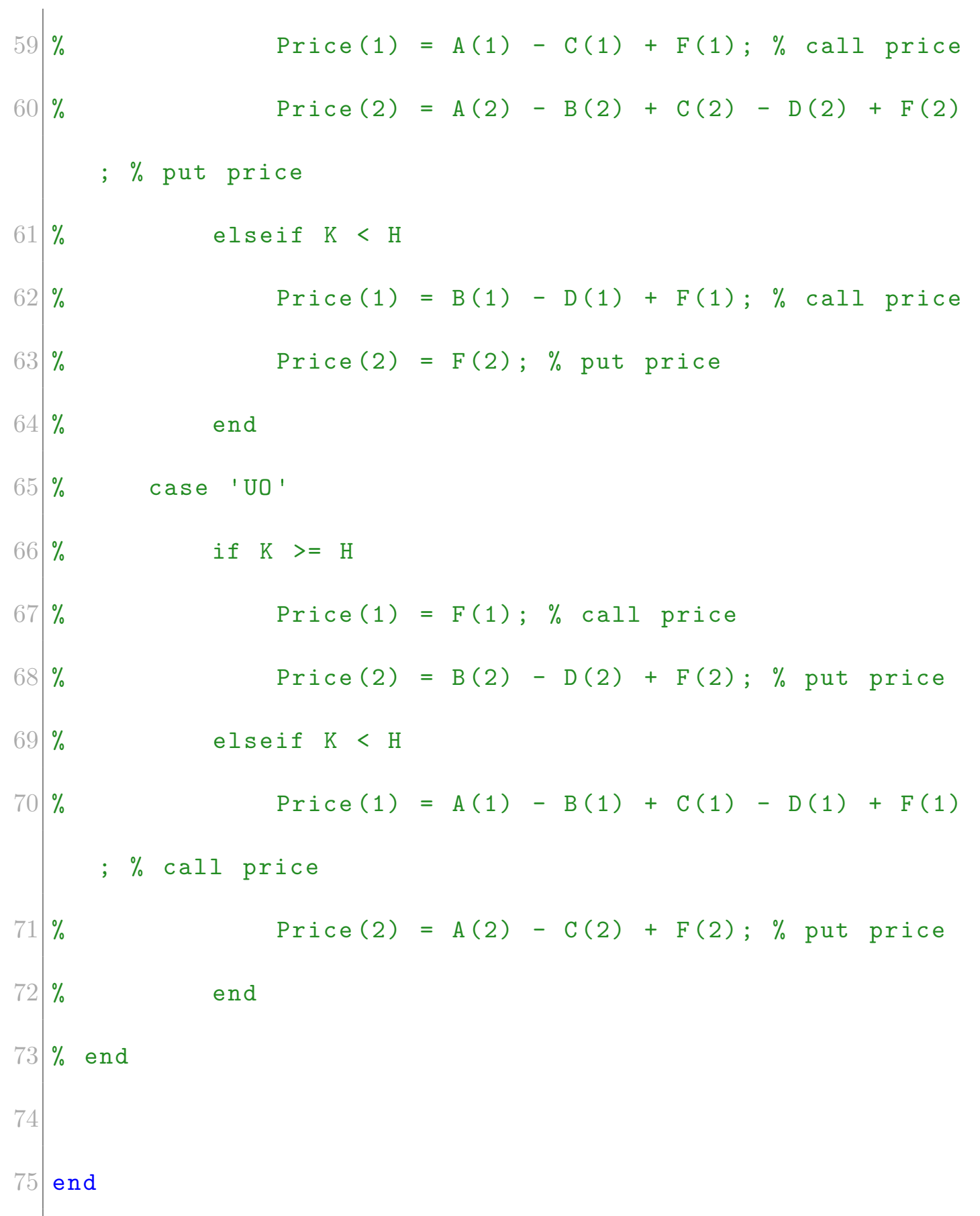

Mainscript Bivariate Black-Scholes Model

close all; 


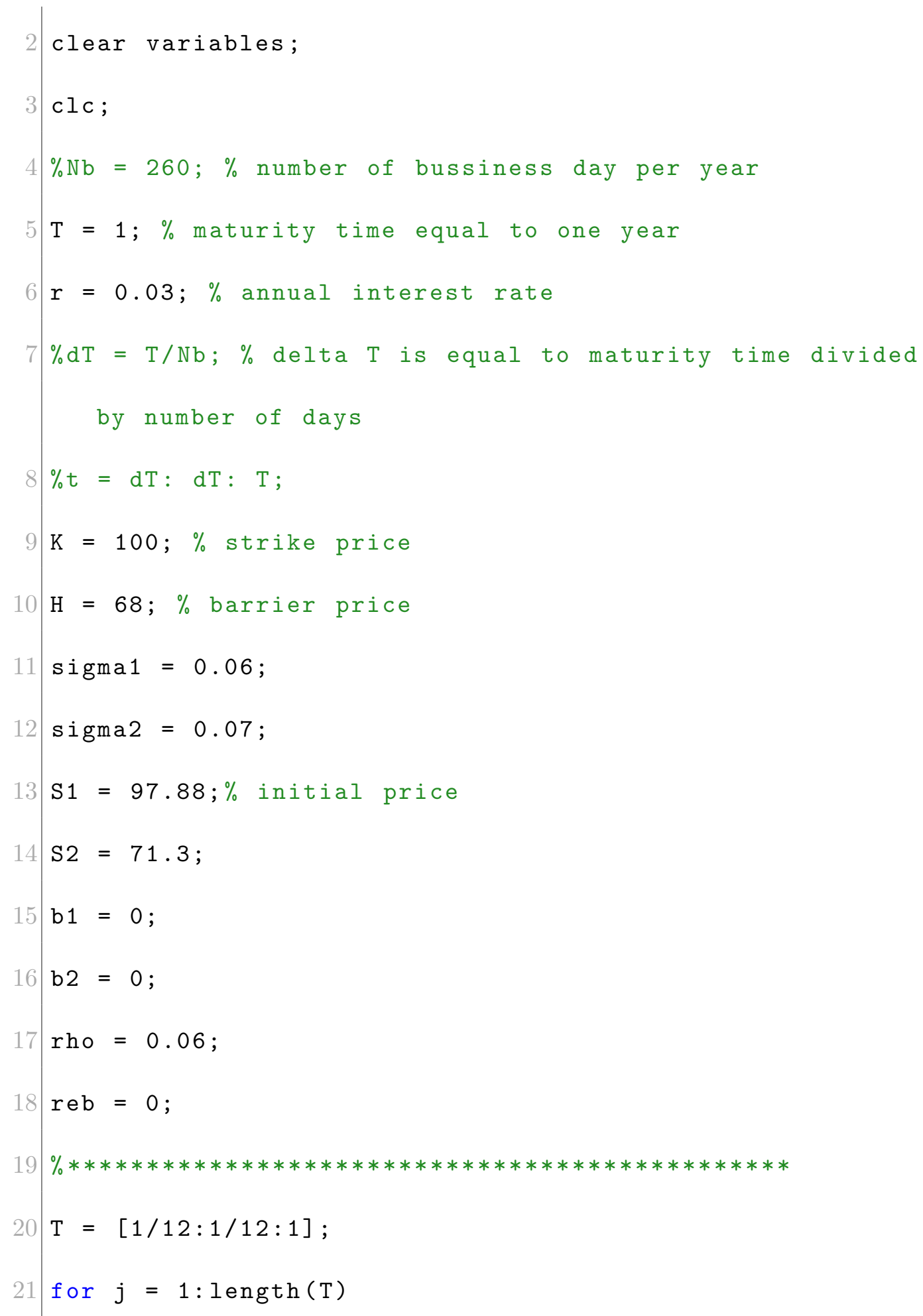




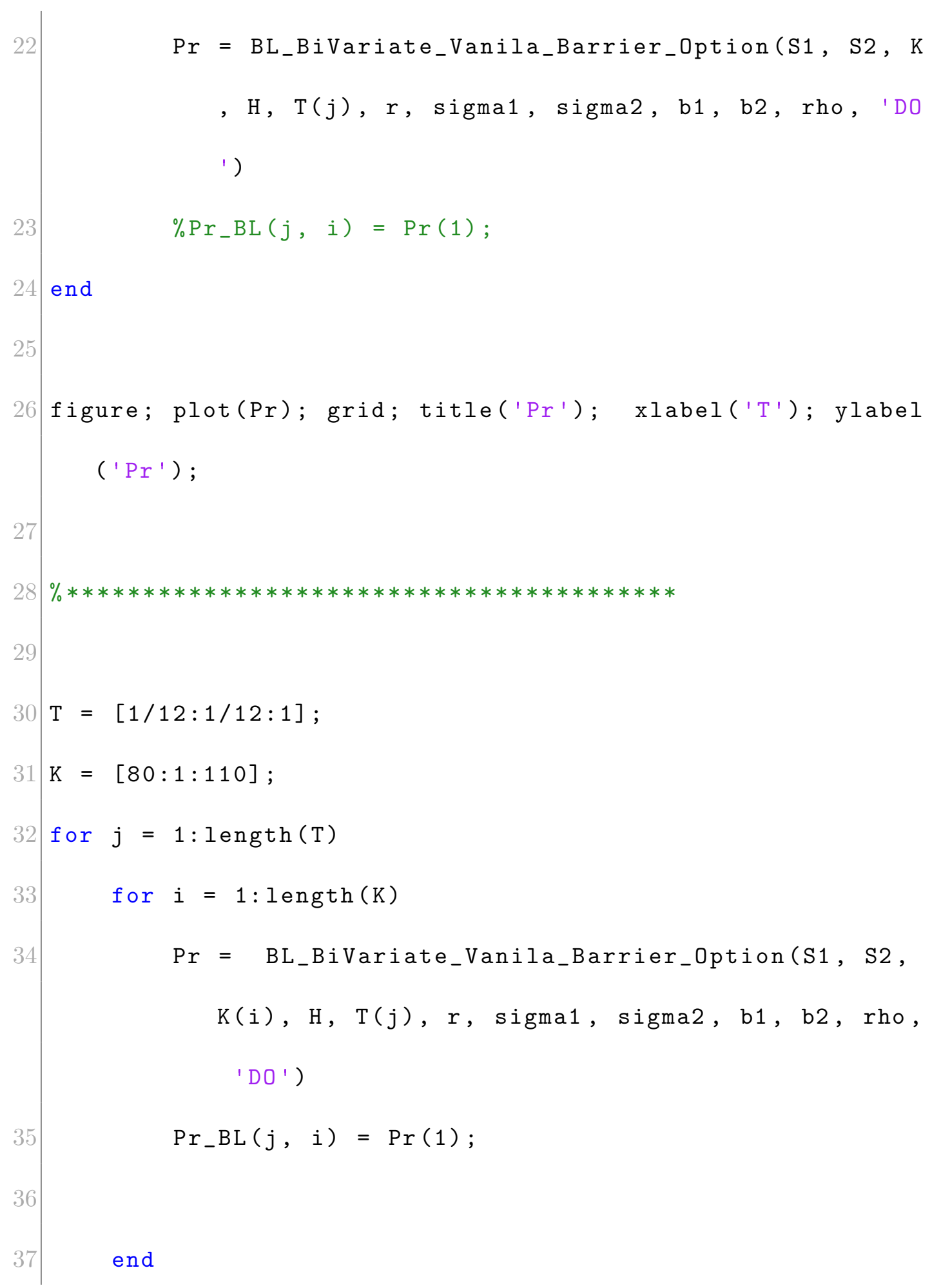




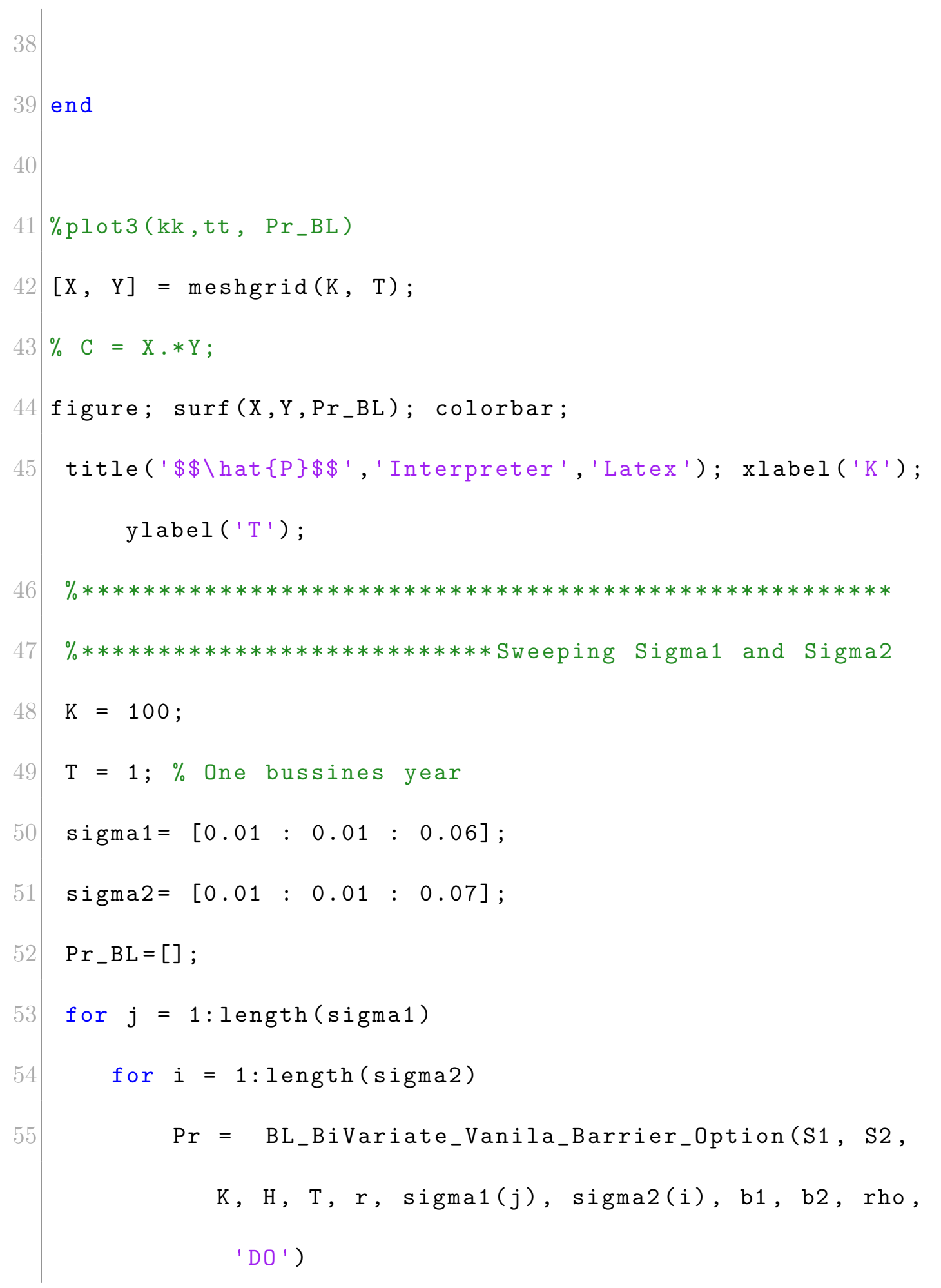




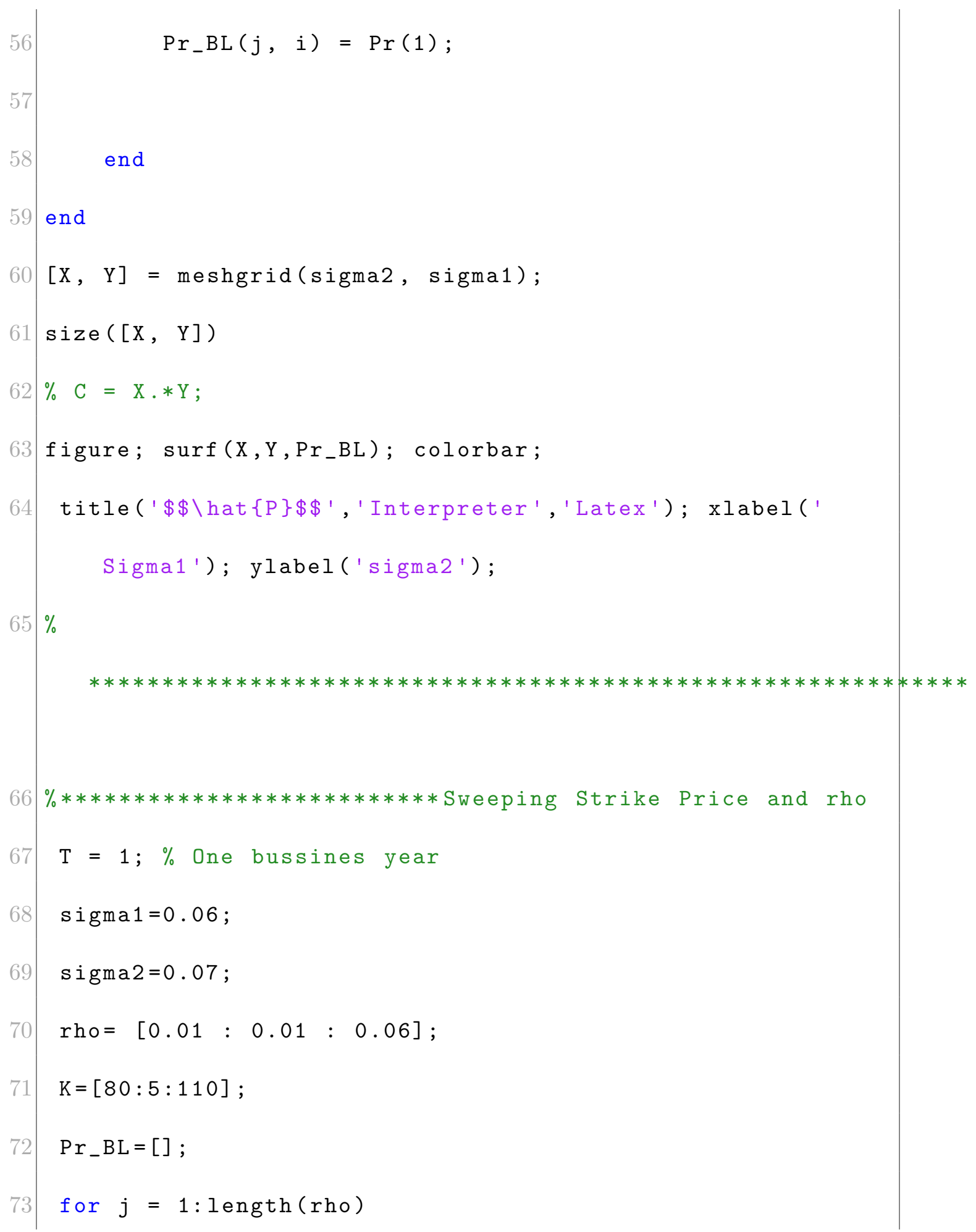




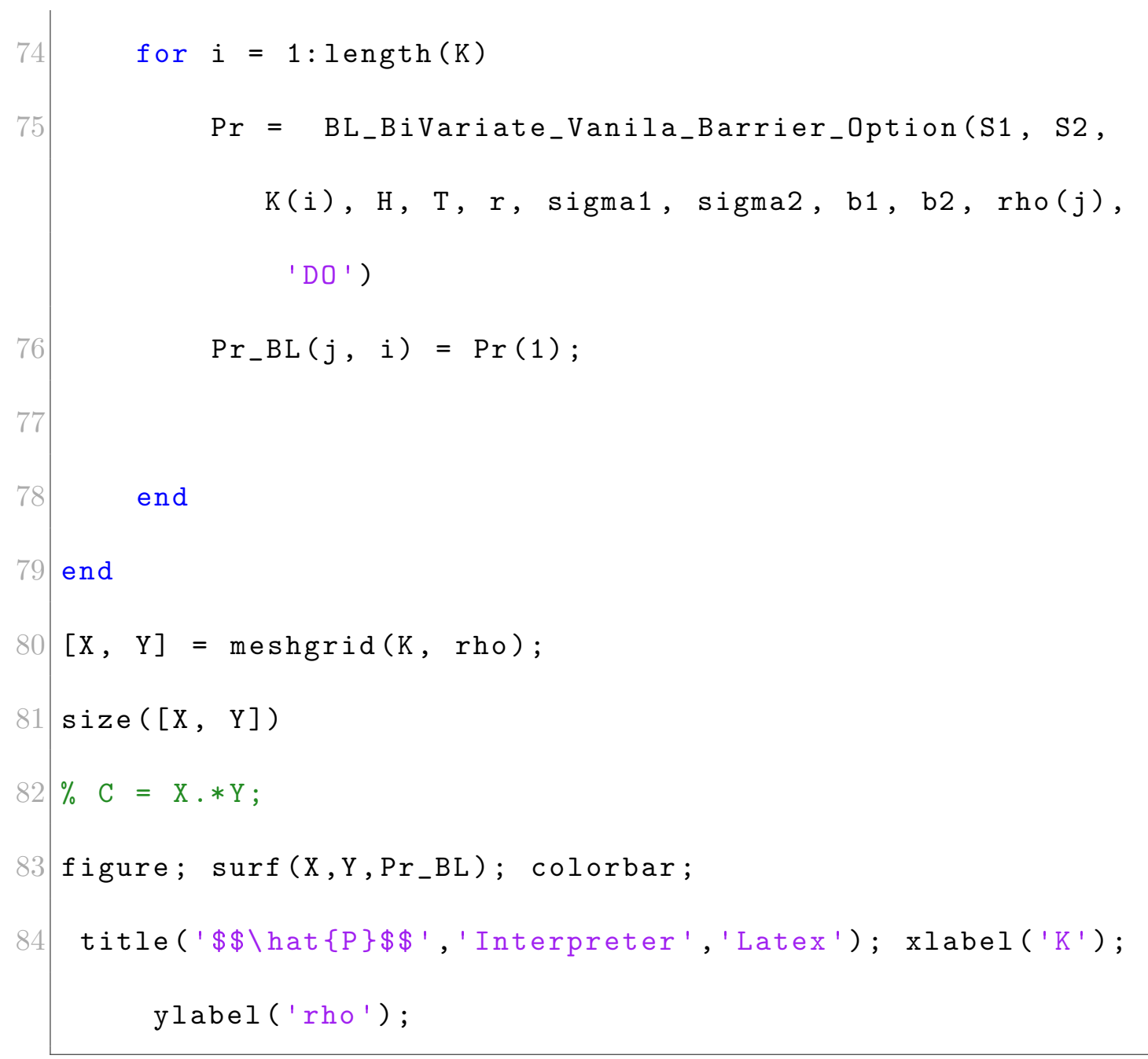

B. Time-Changed Model B.1 Univariate Time-Changed Model Inverse Gaussian Subordinator

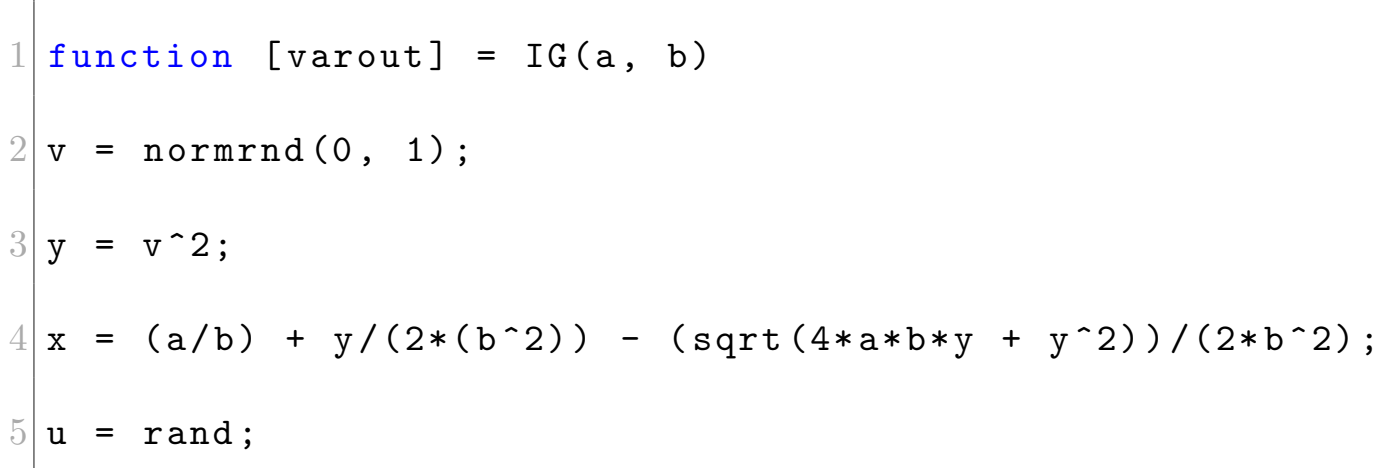




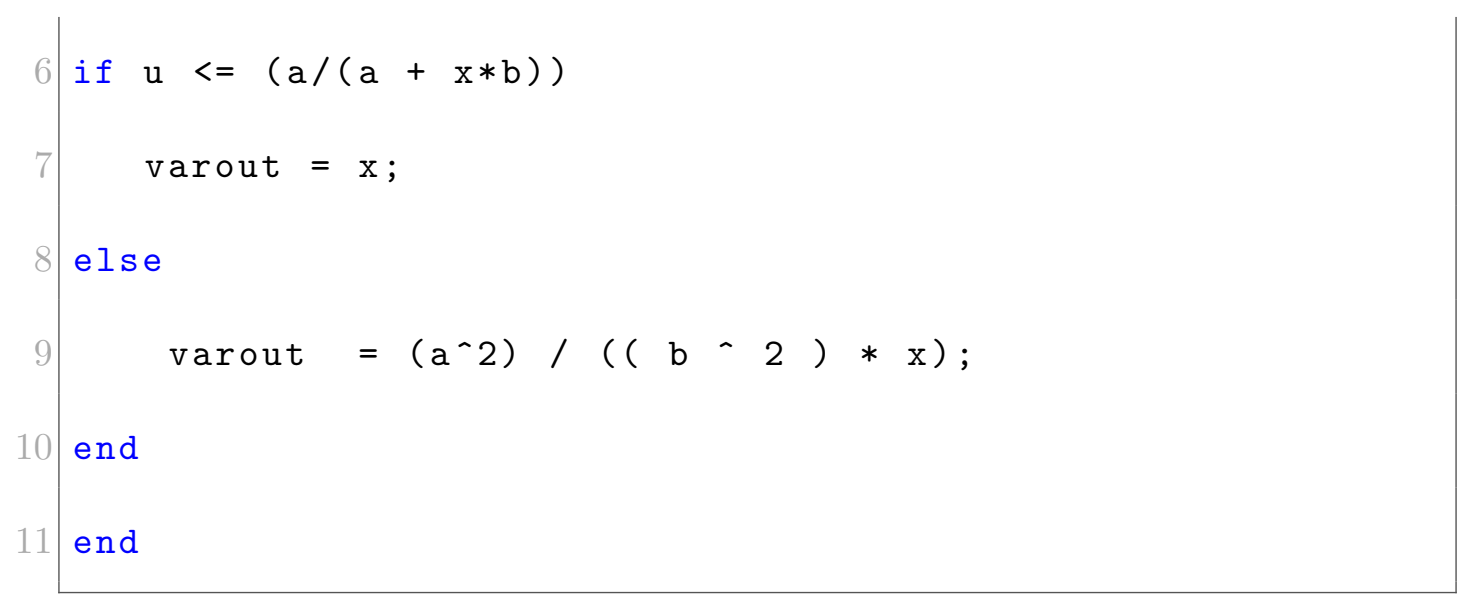

\section{Barrier Price Option}

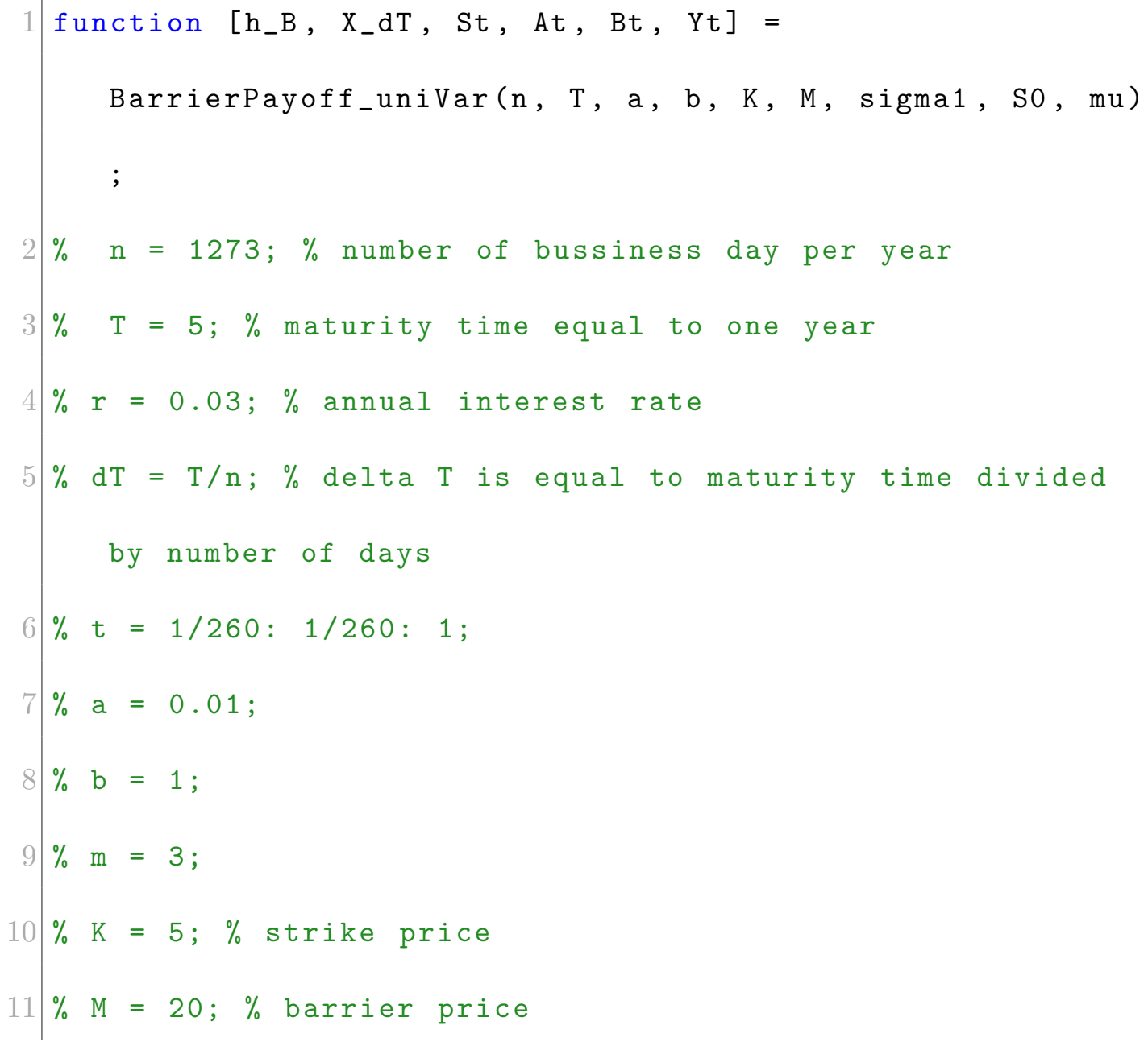




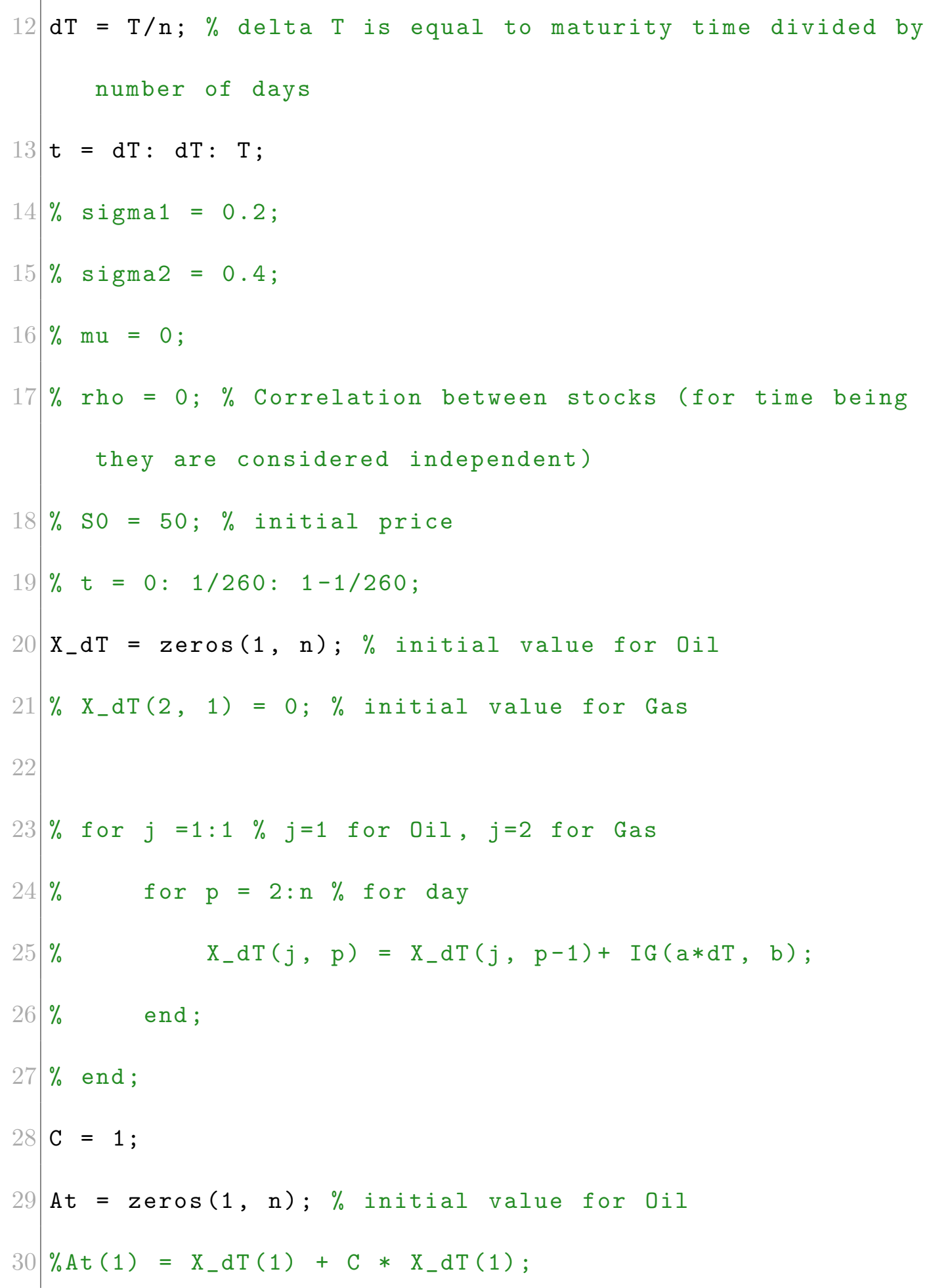




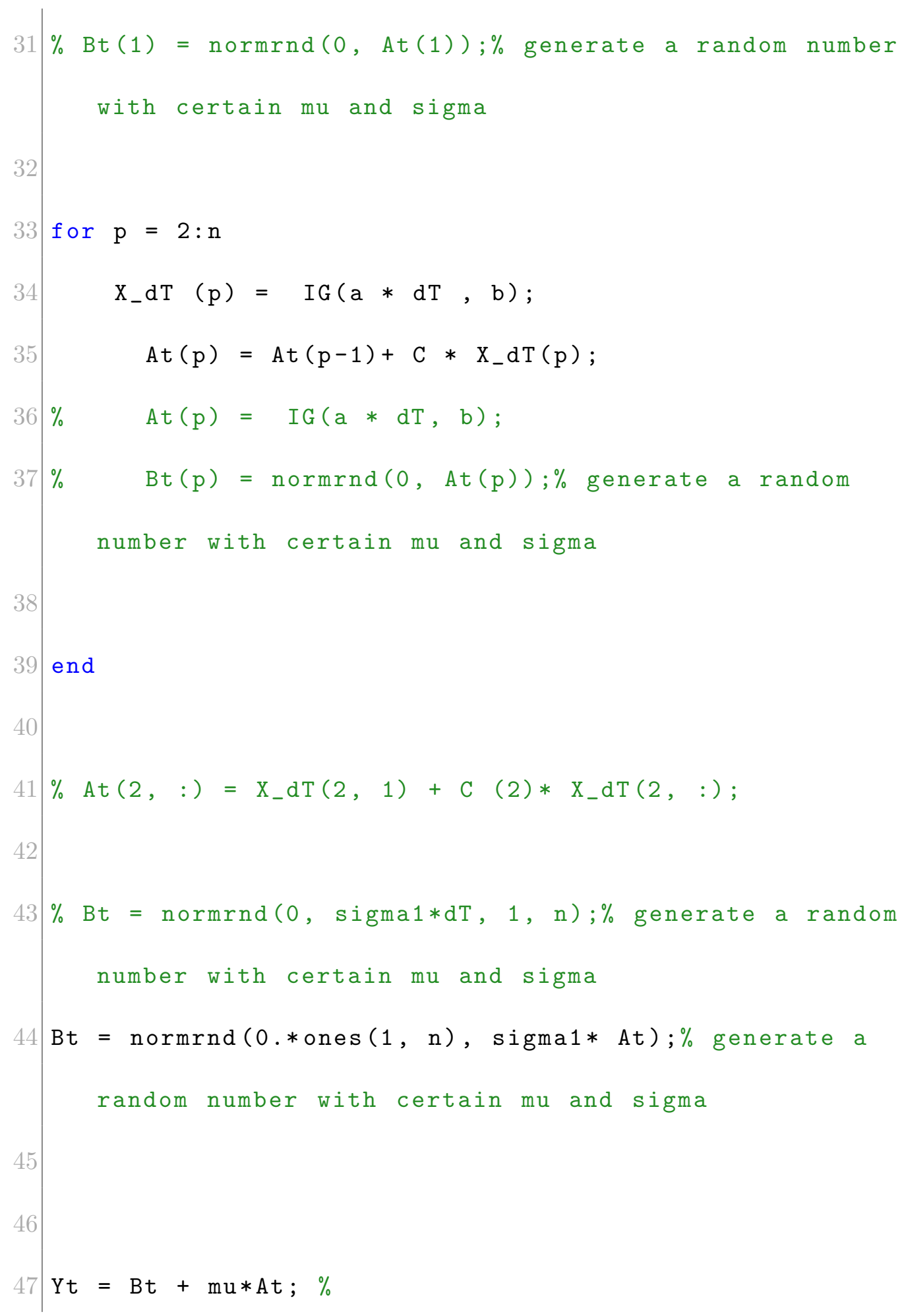




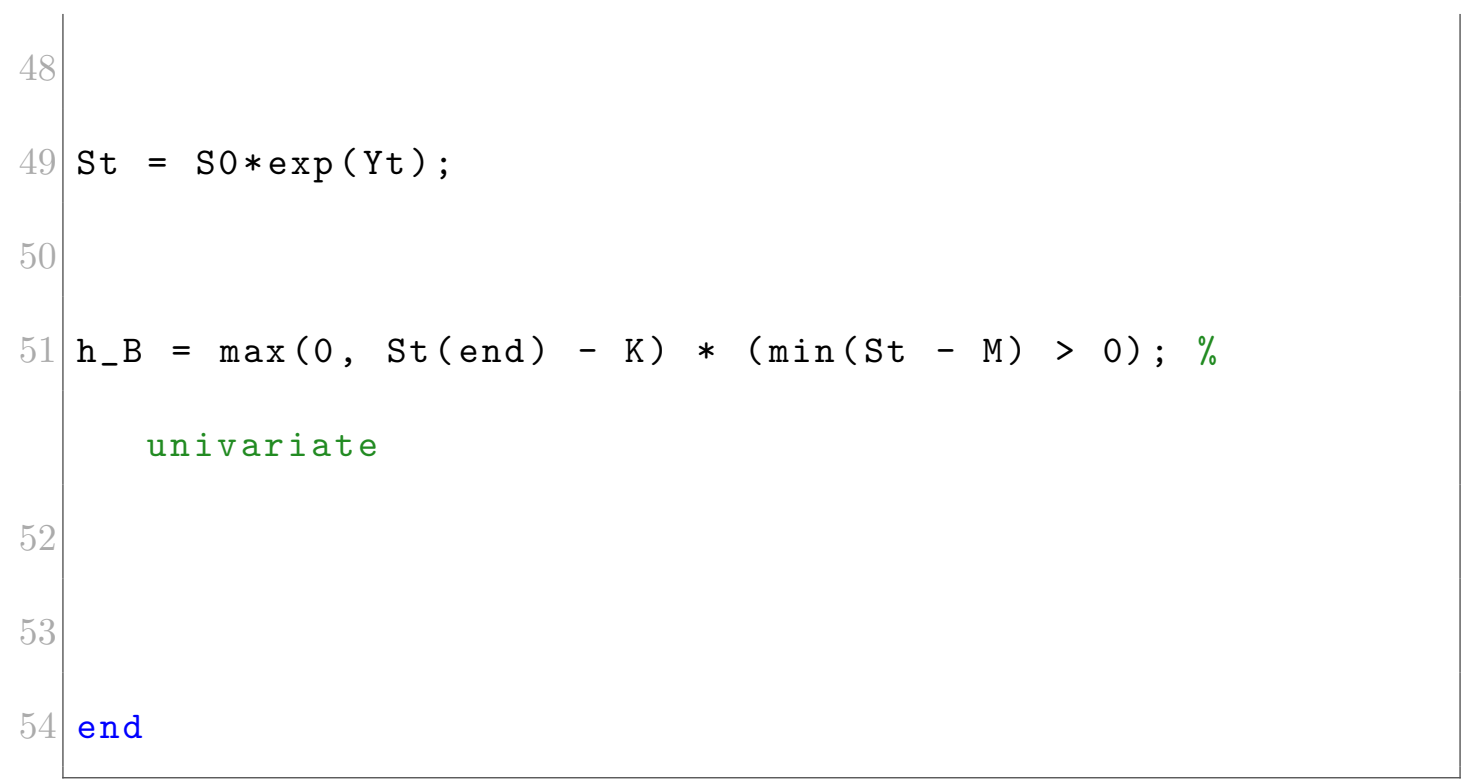

Monte Carlo Method

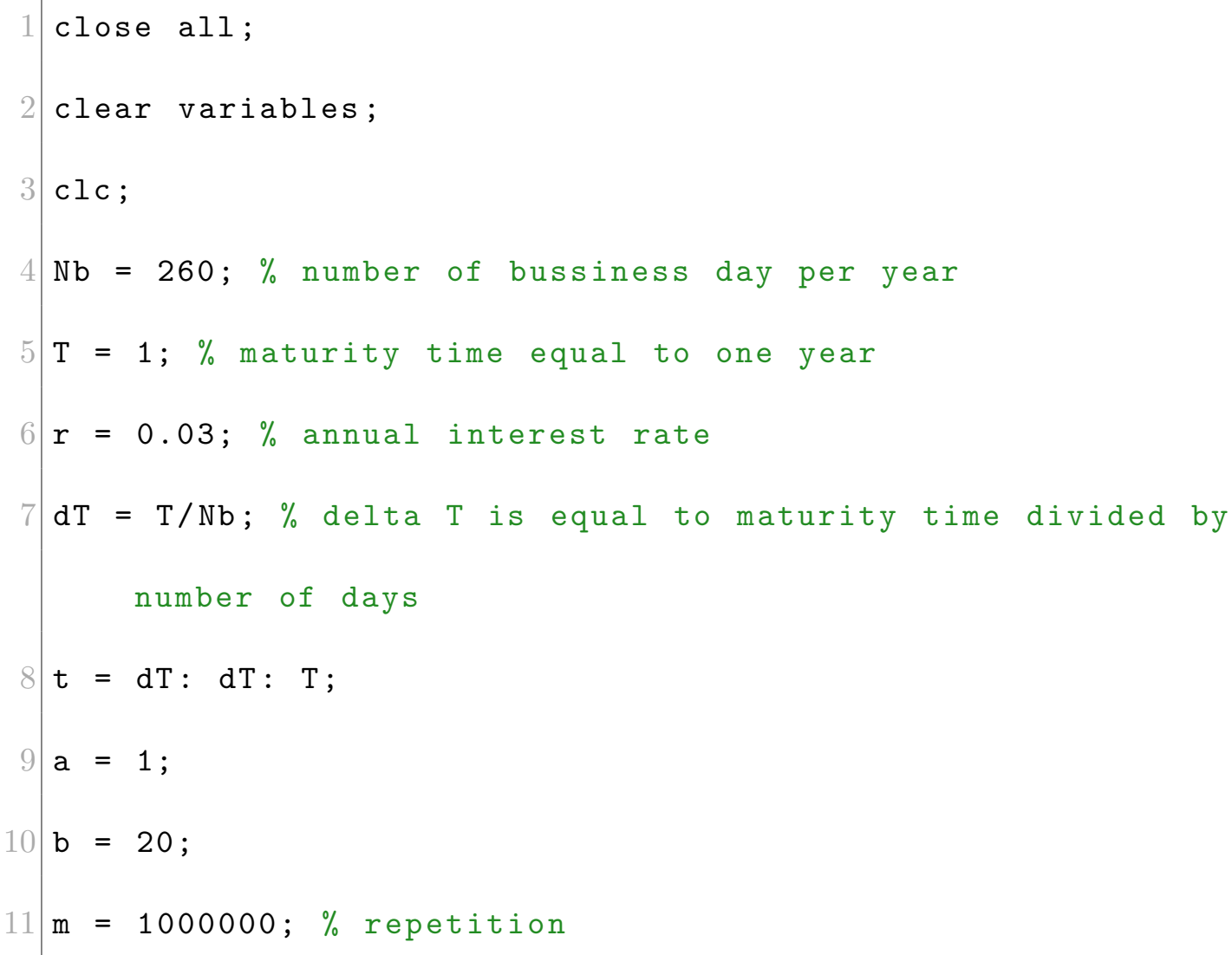




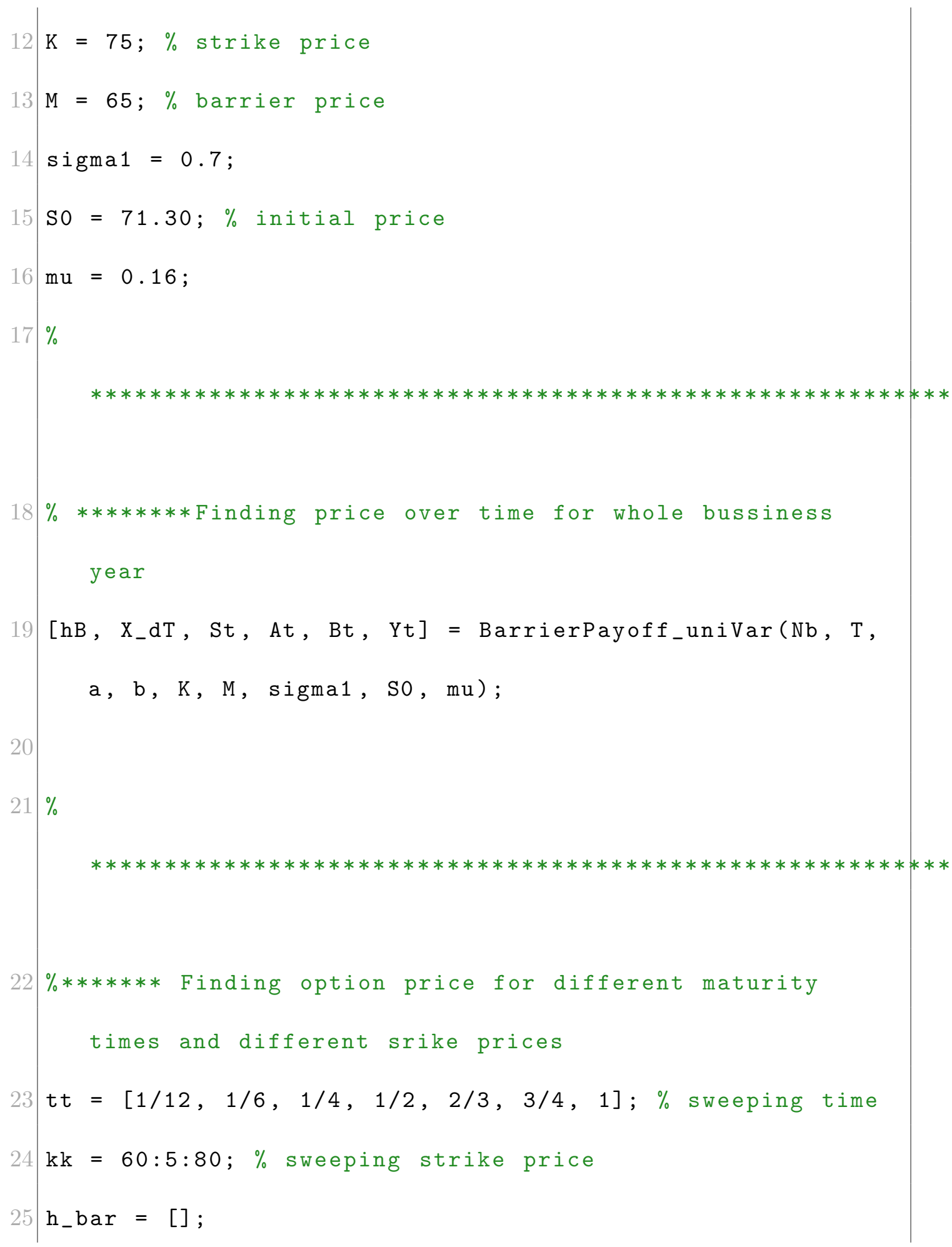




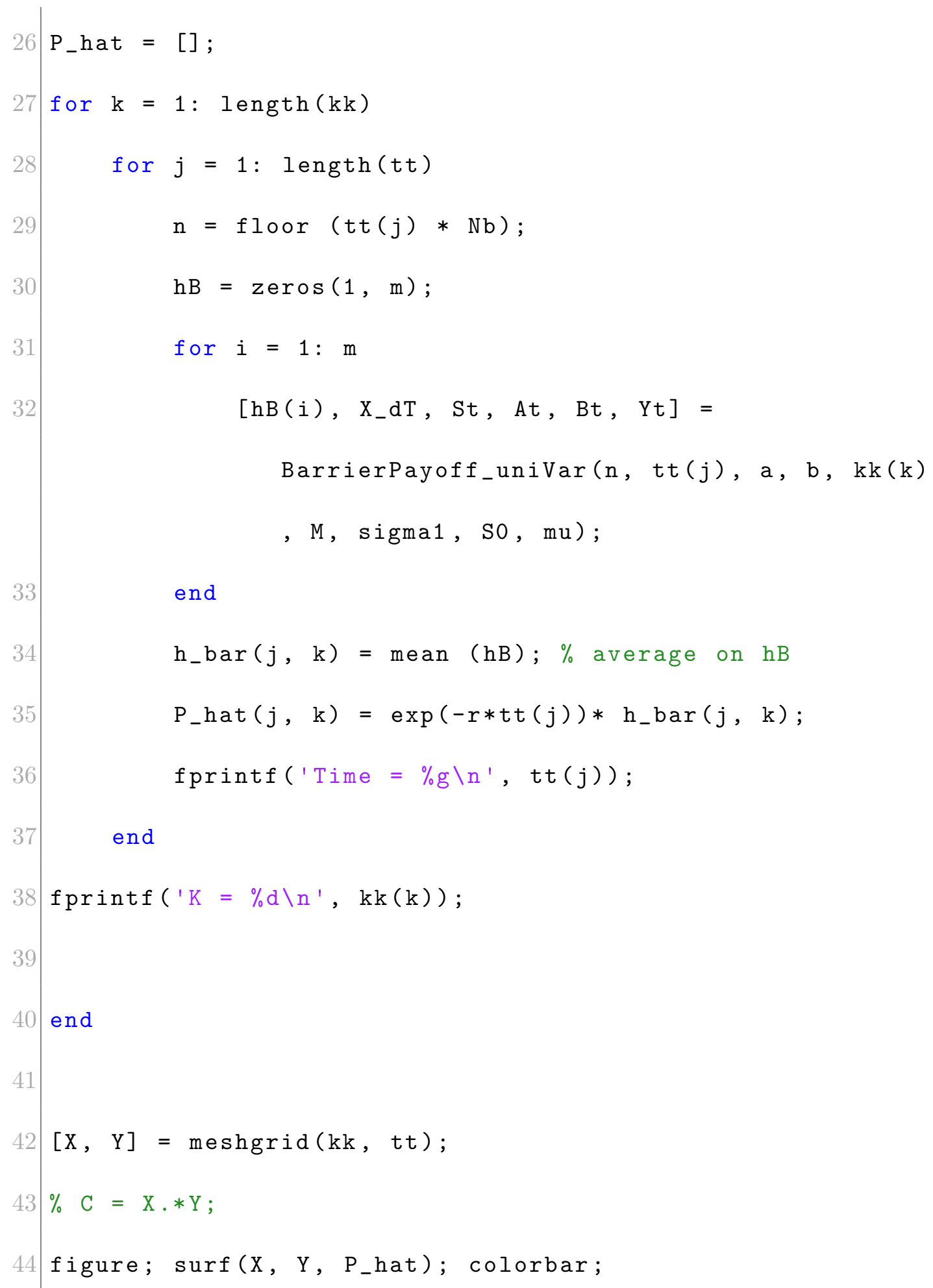




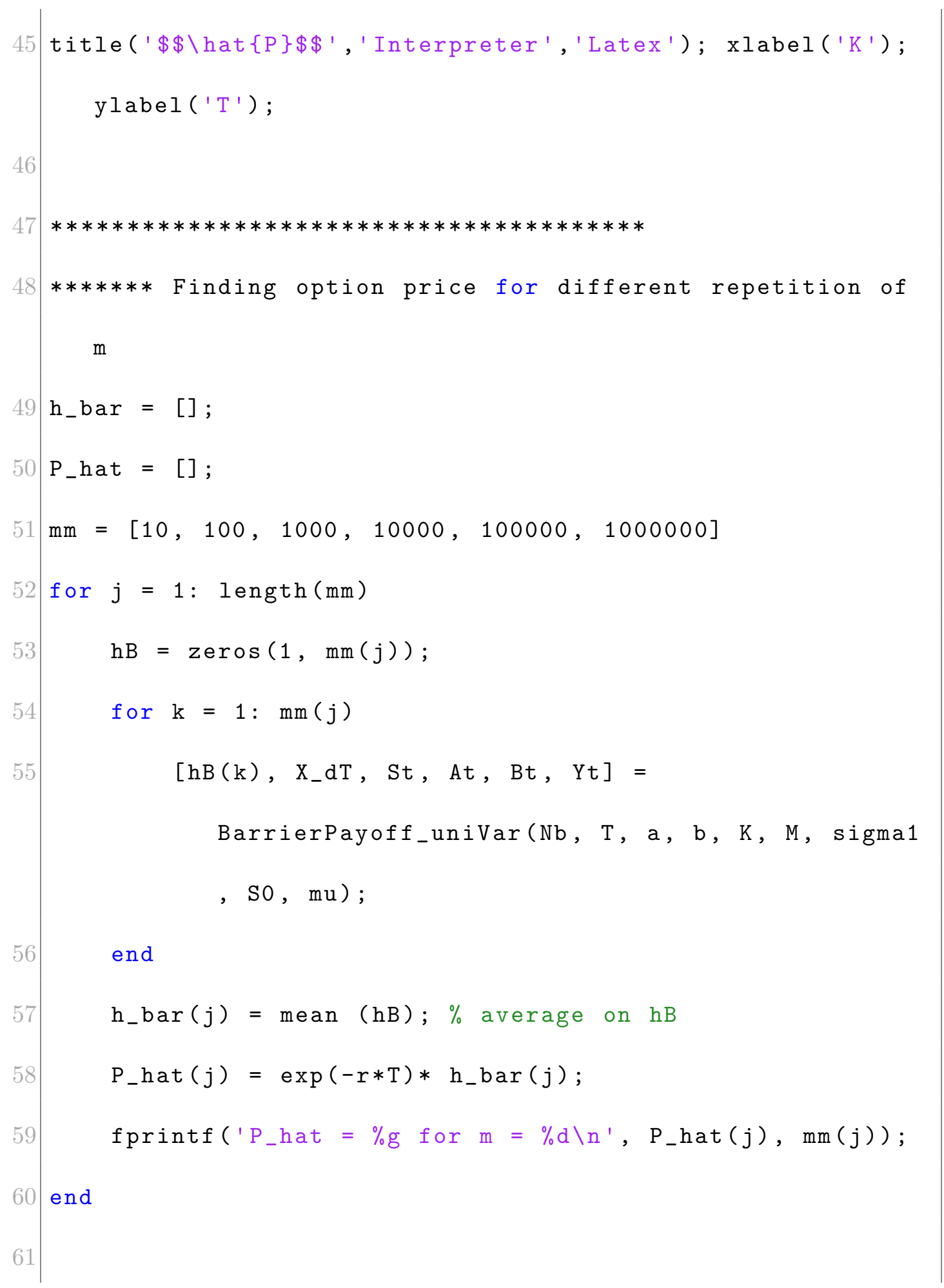




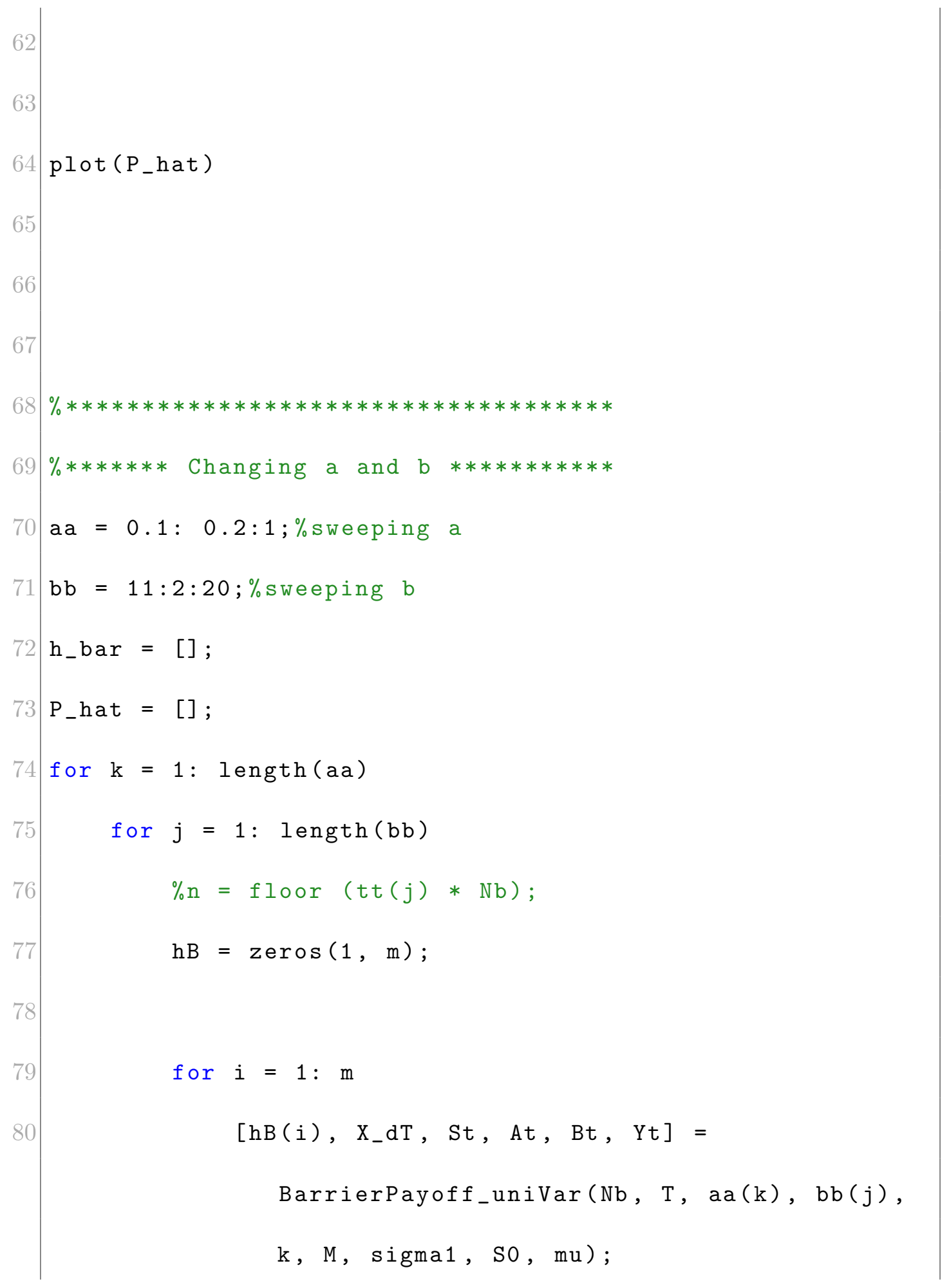




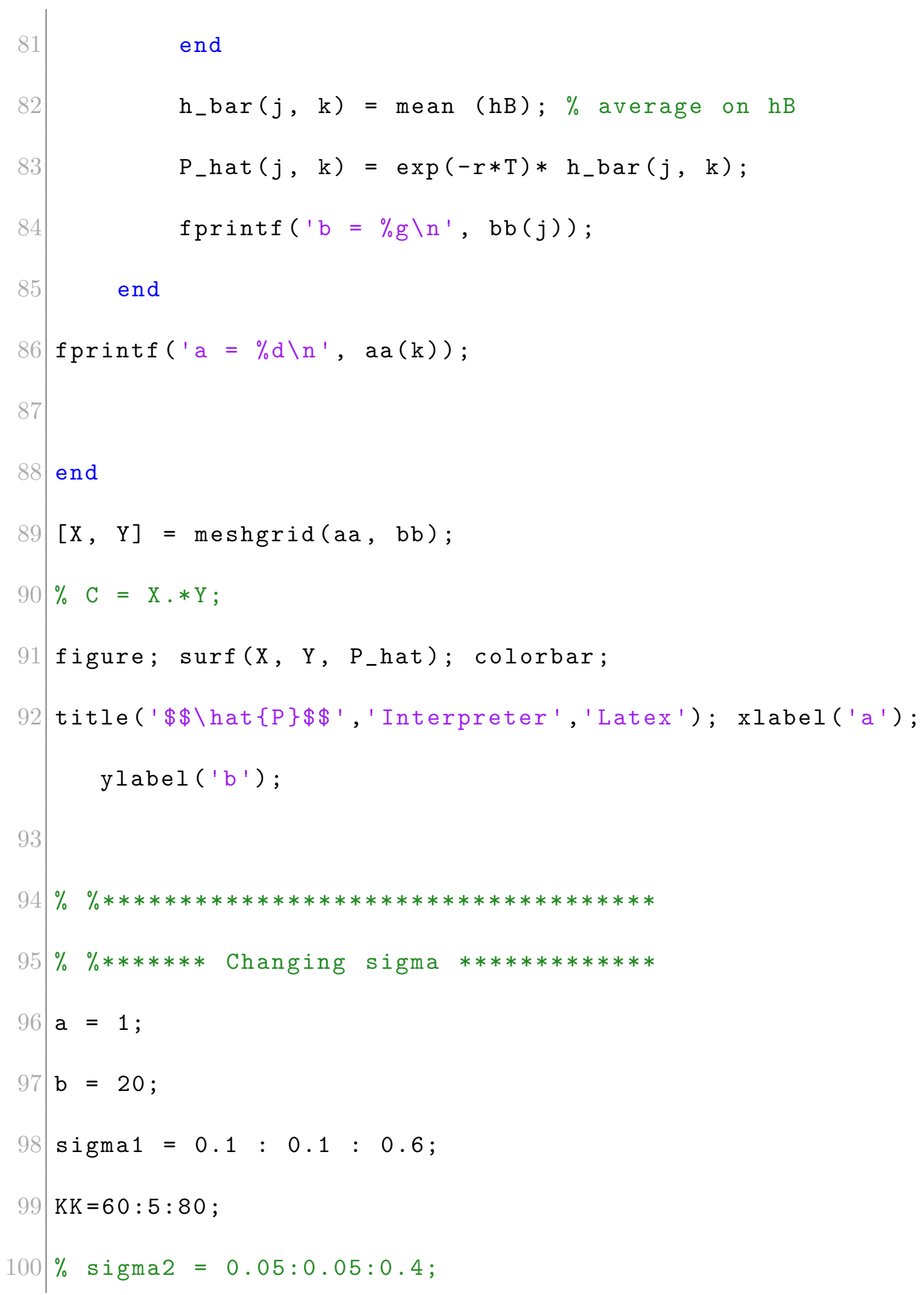




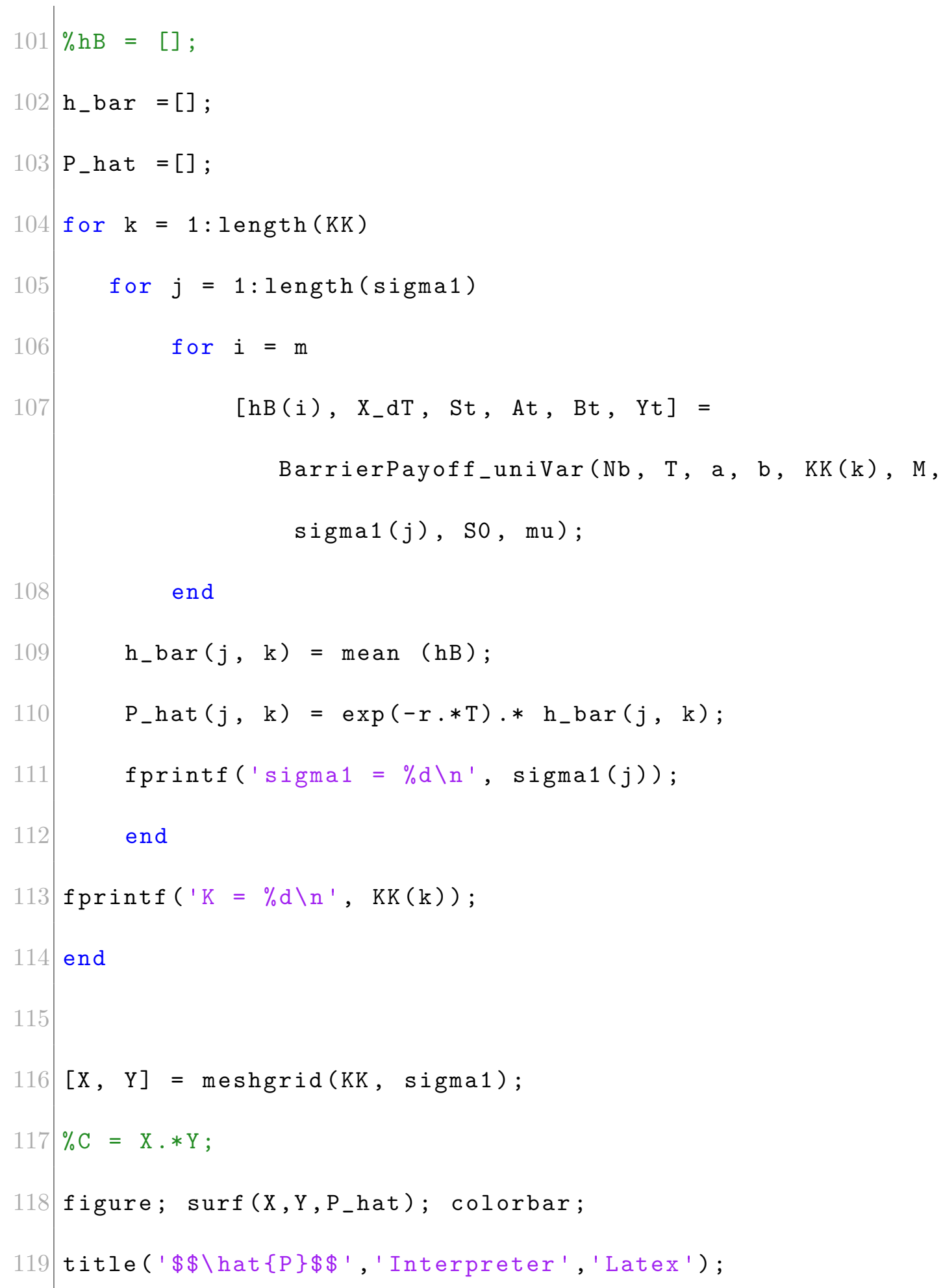




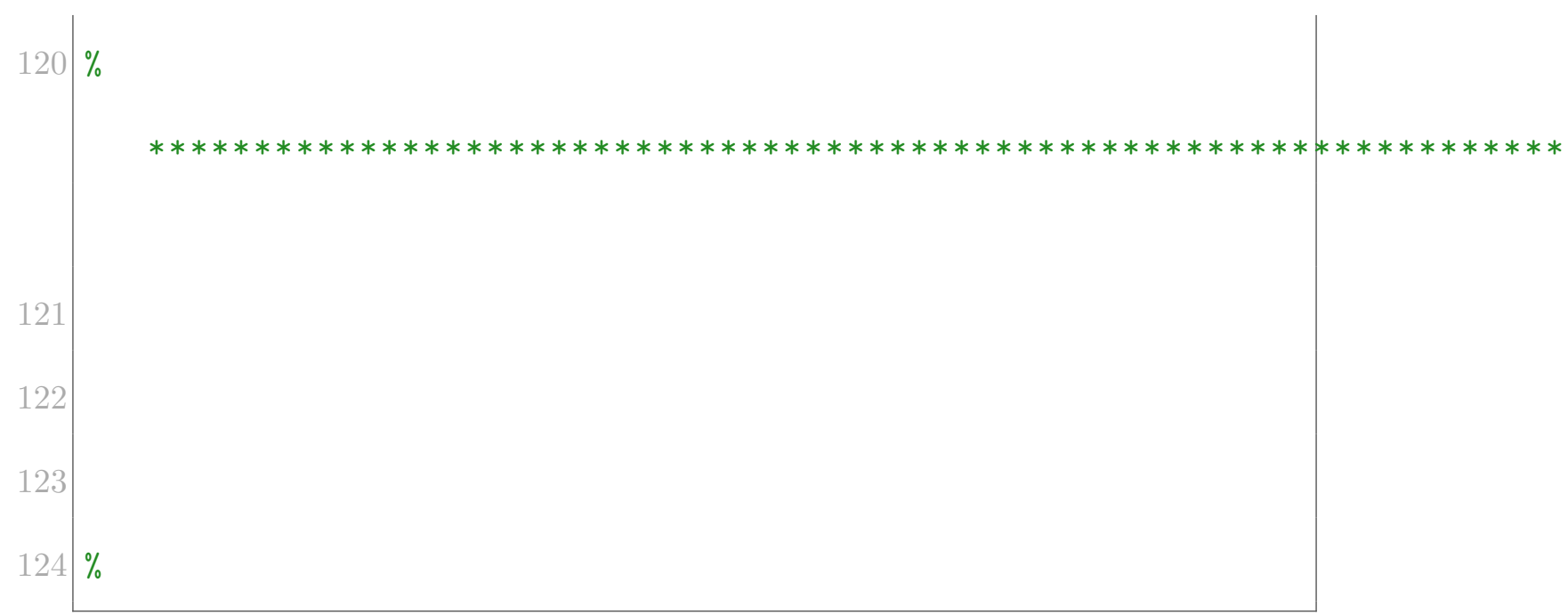

B.2 Bivariate Time-Changed Model Barrier Price Option

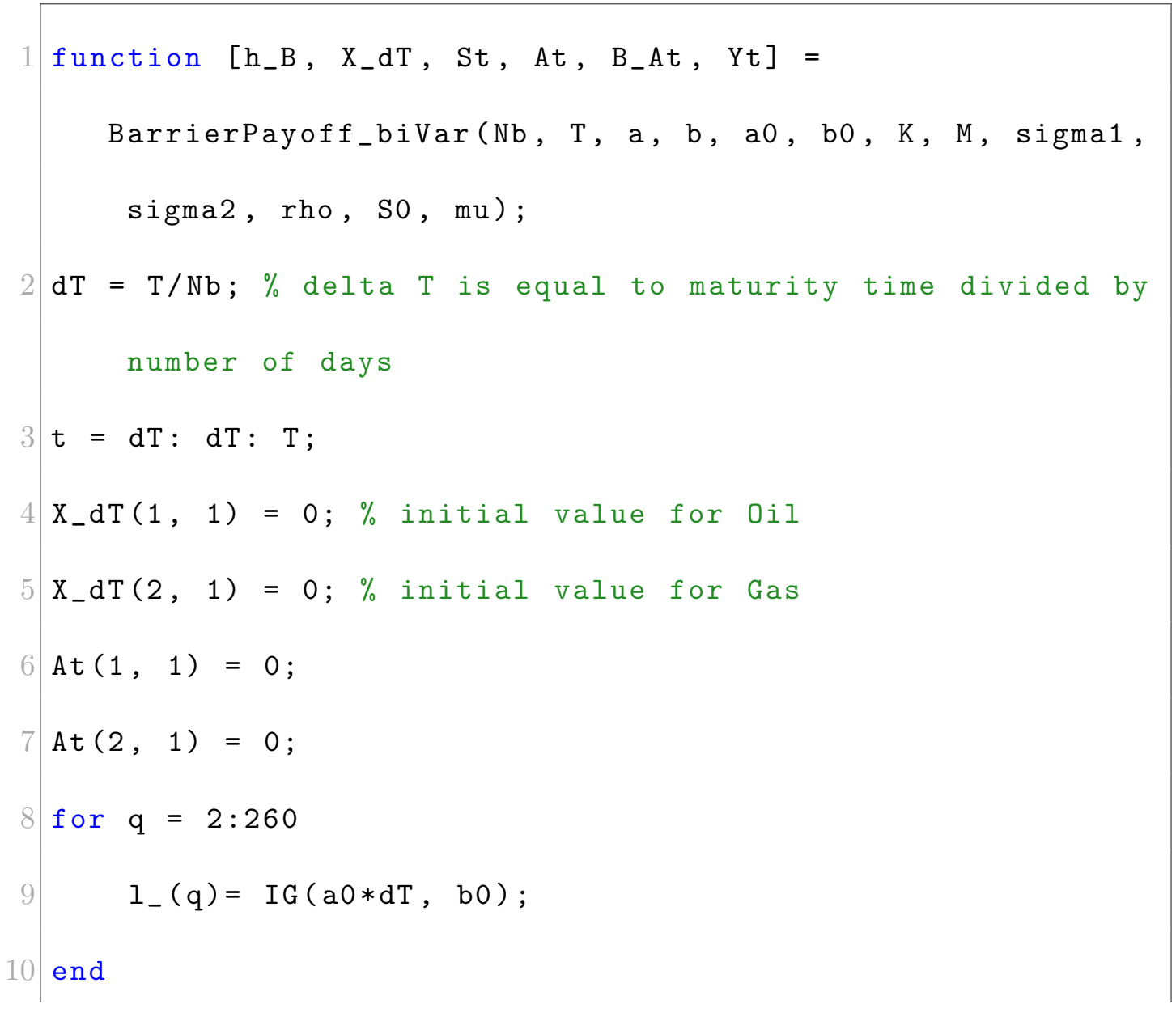




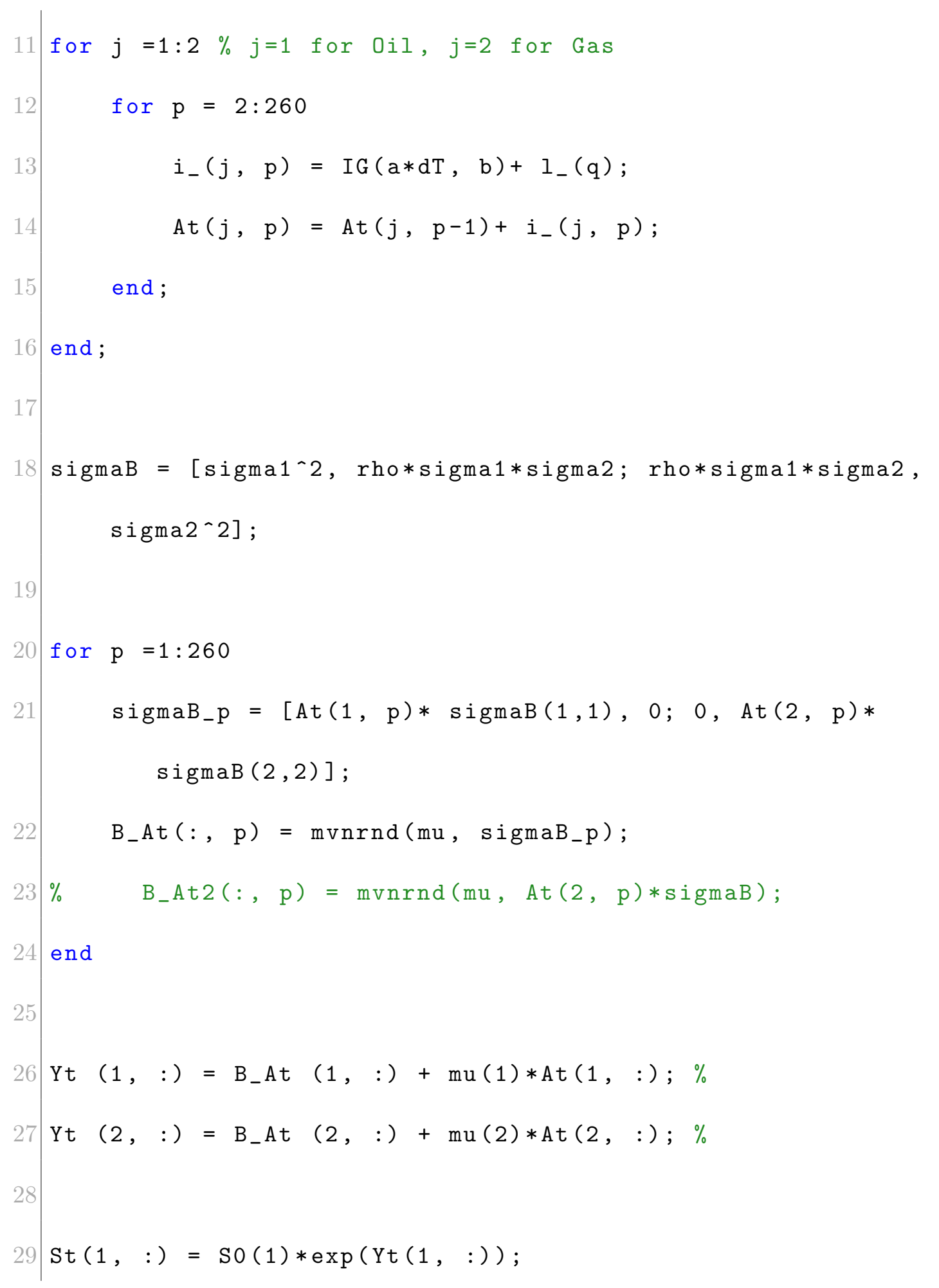




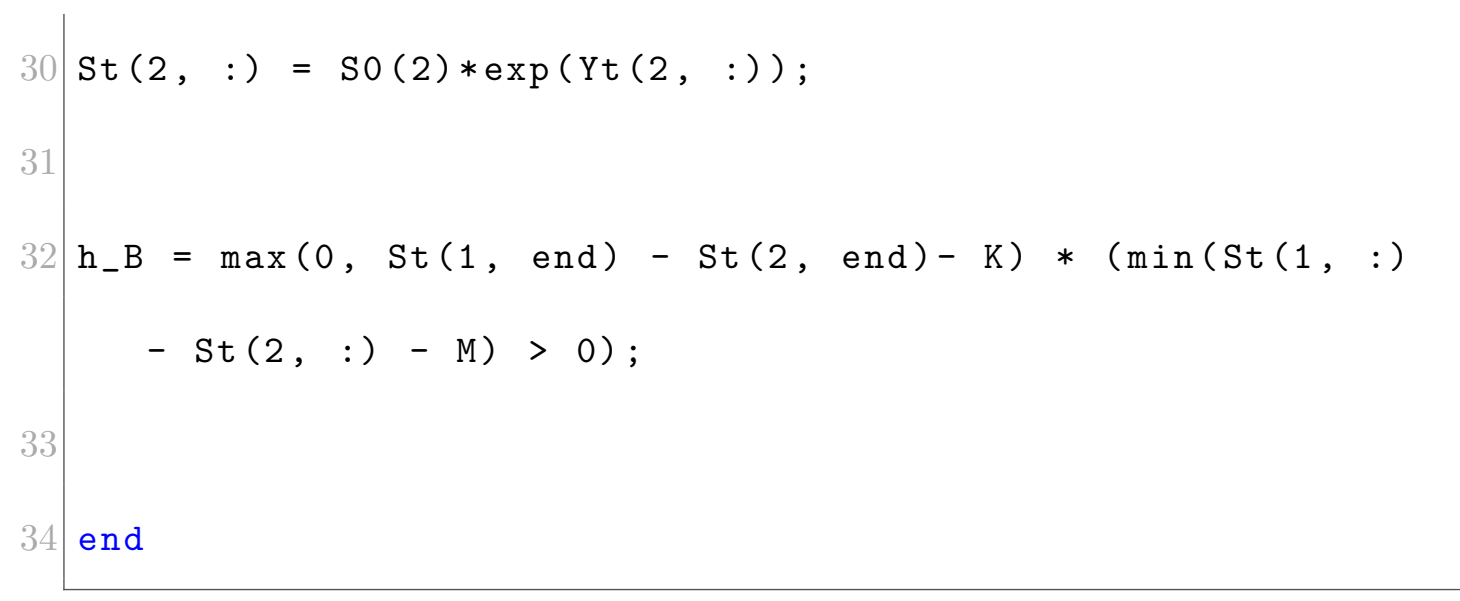

\section{Monte Carlo Method}

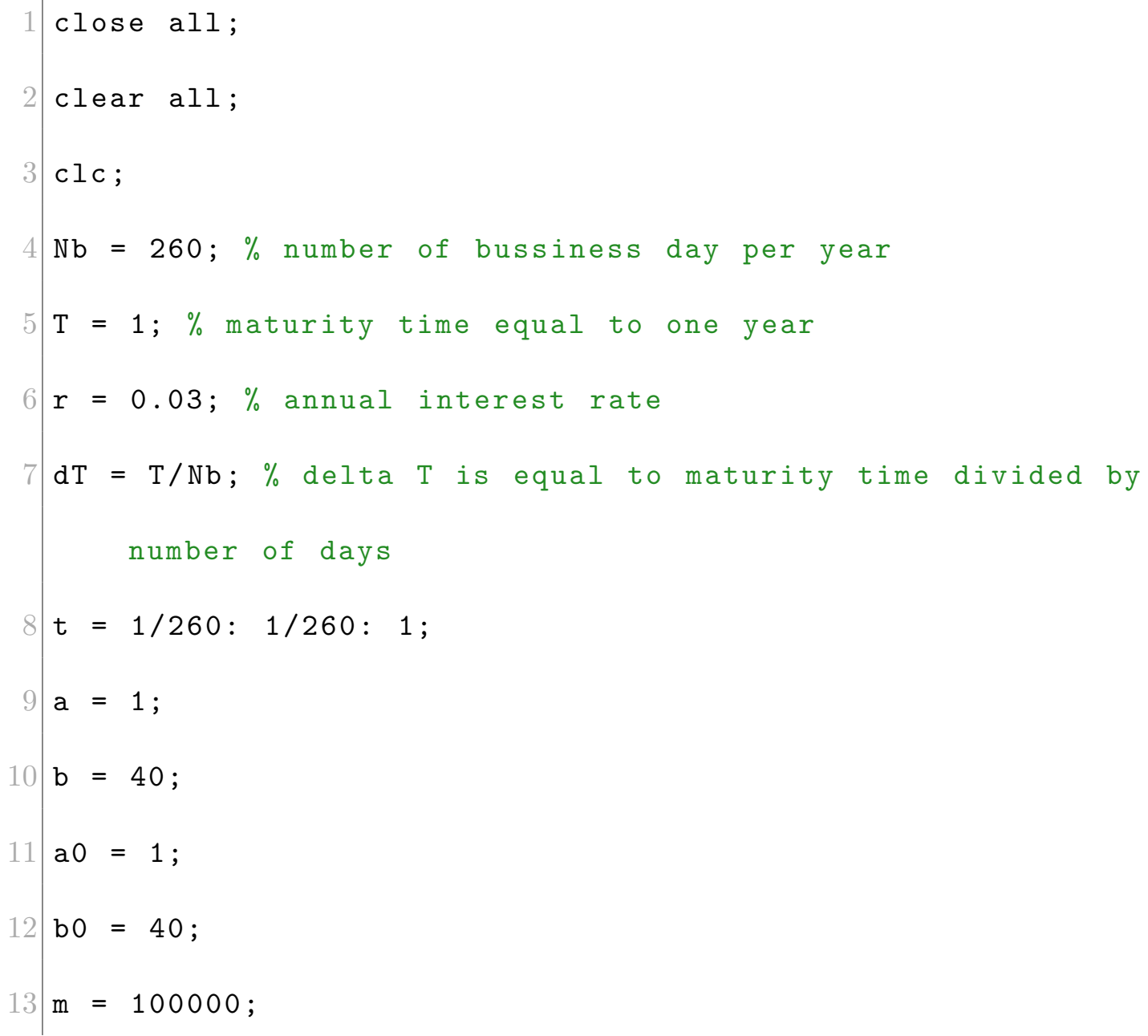




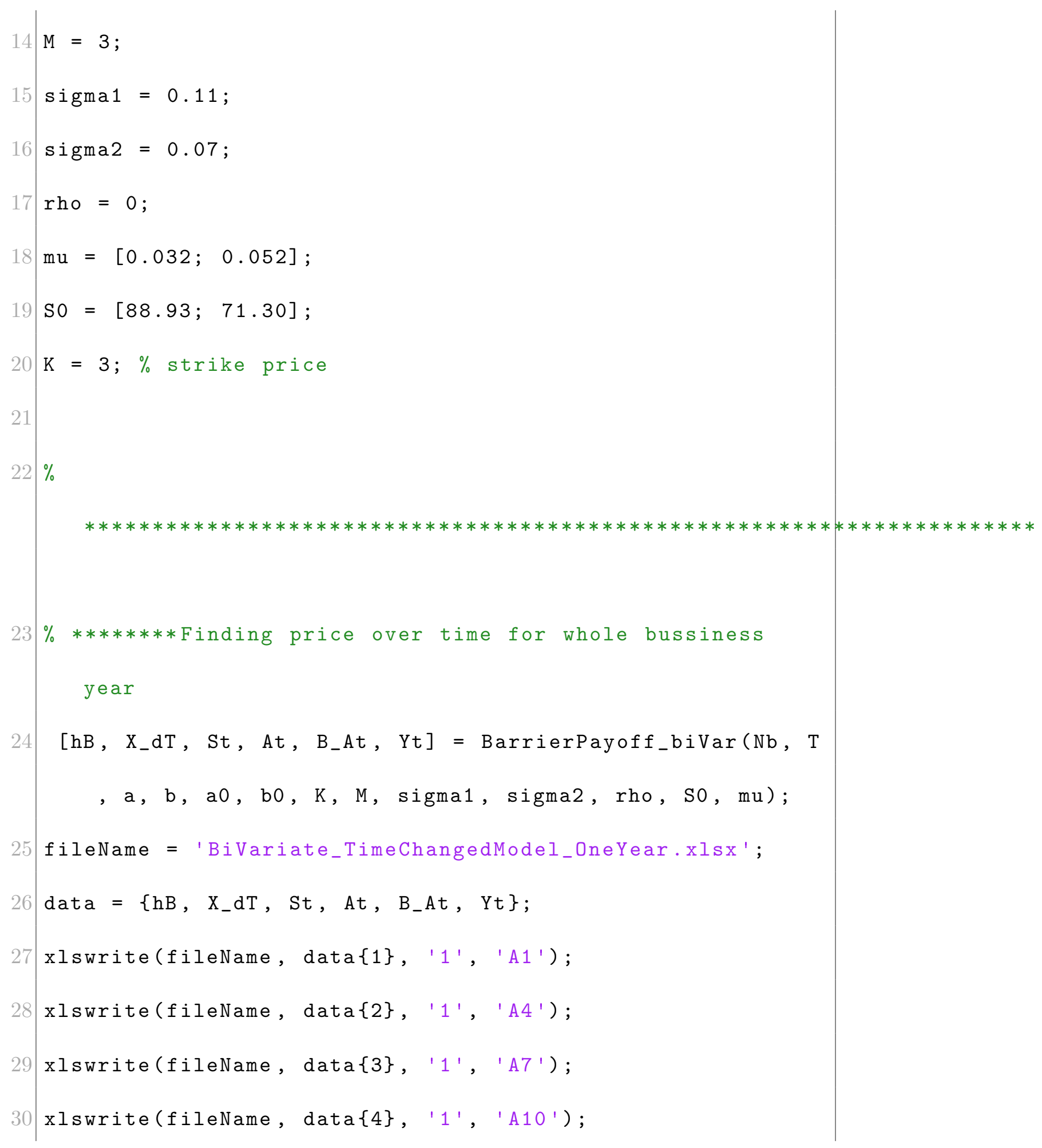




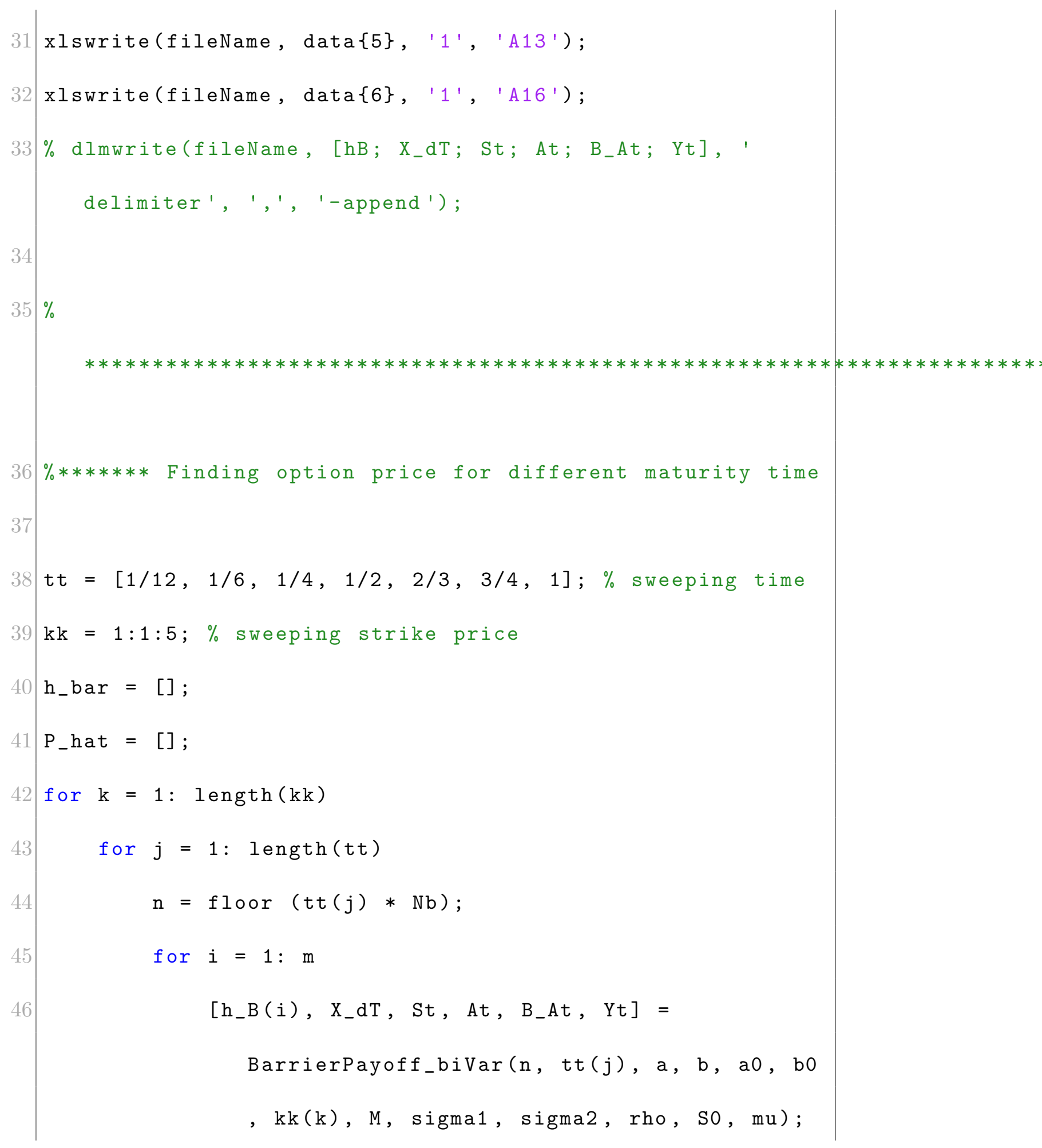




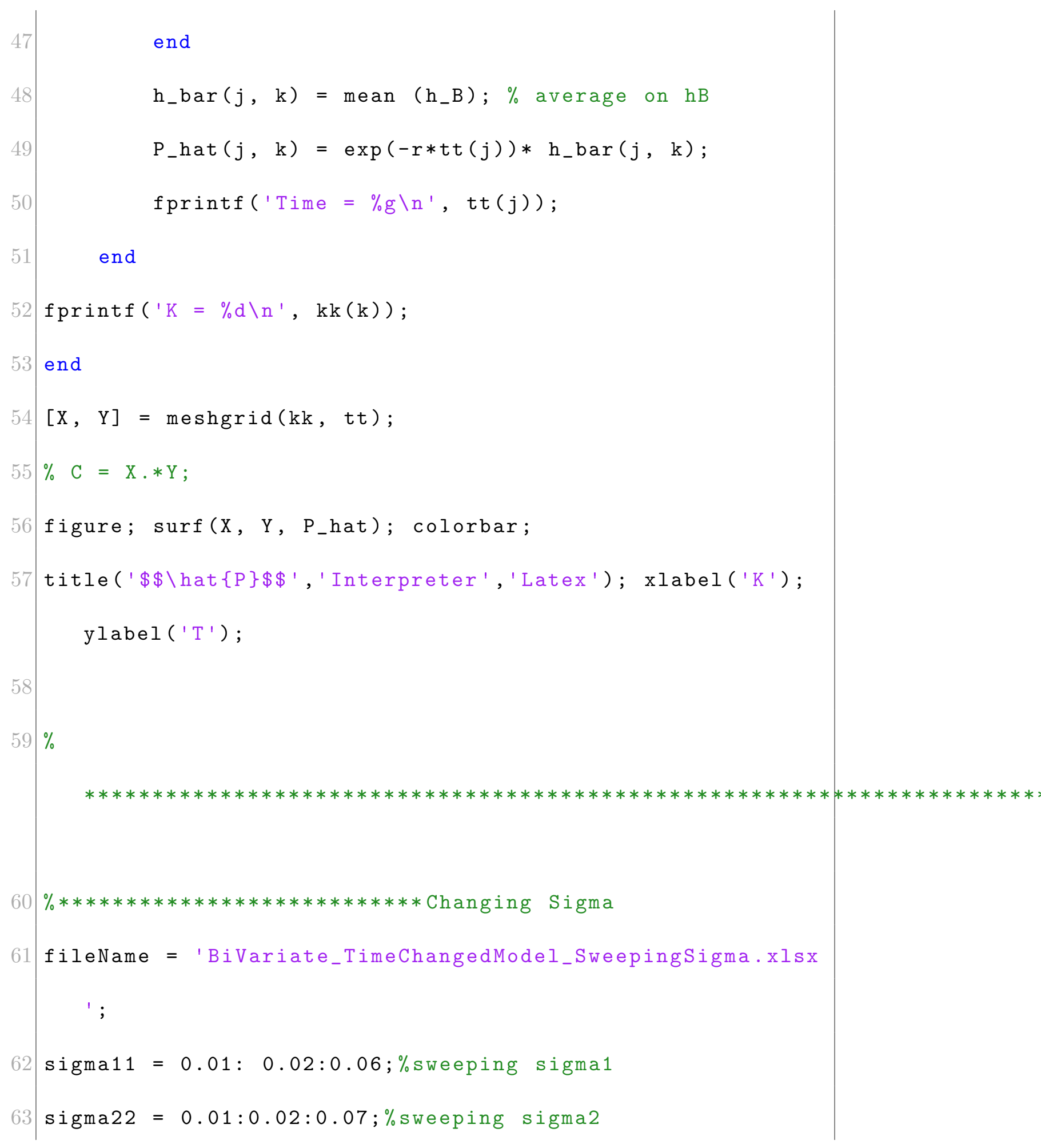




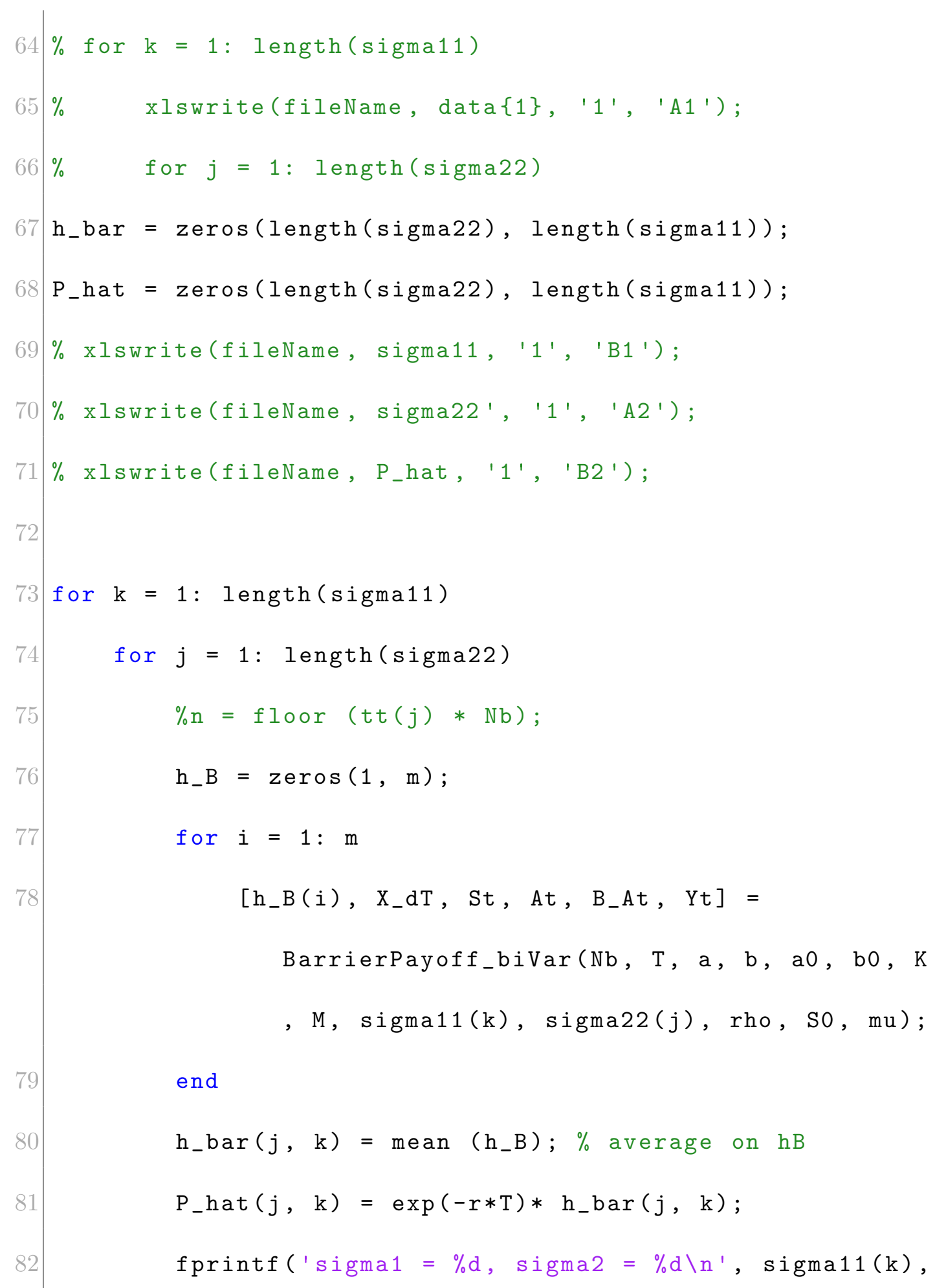




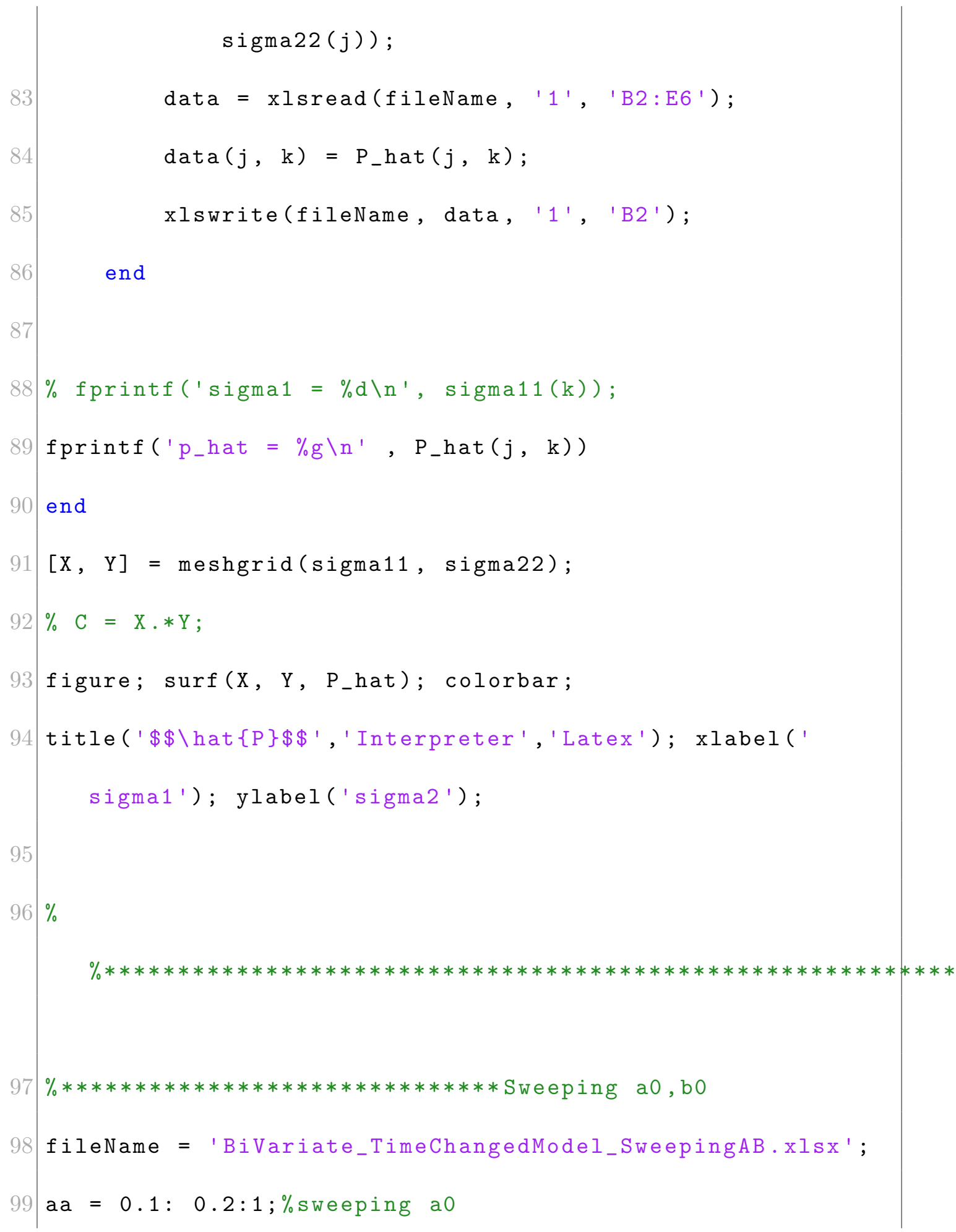




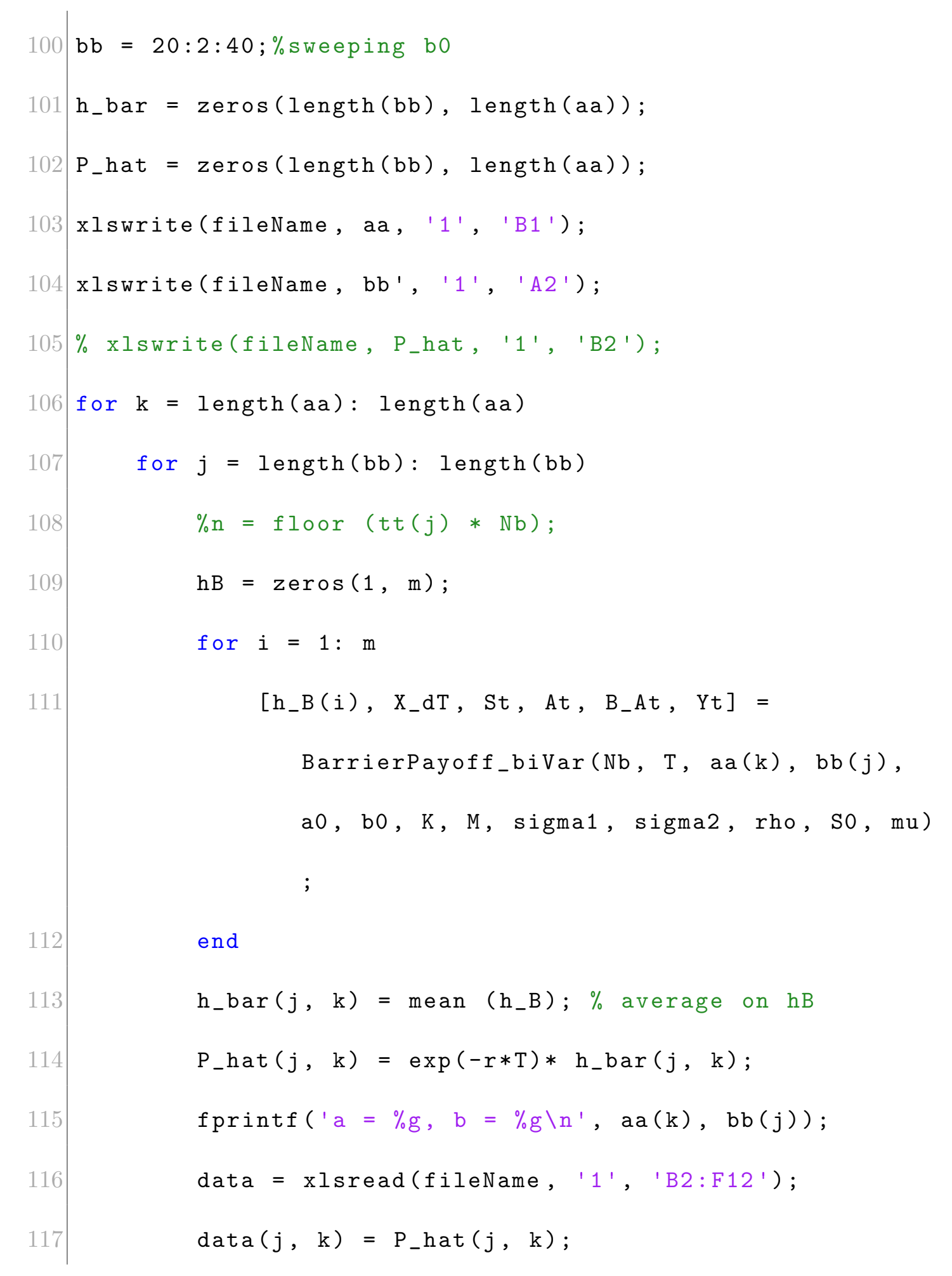




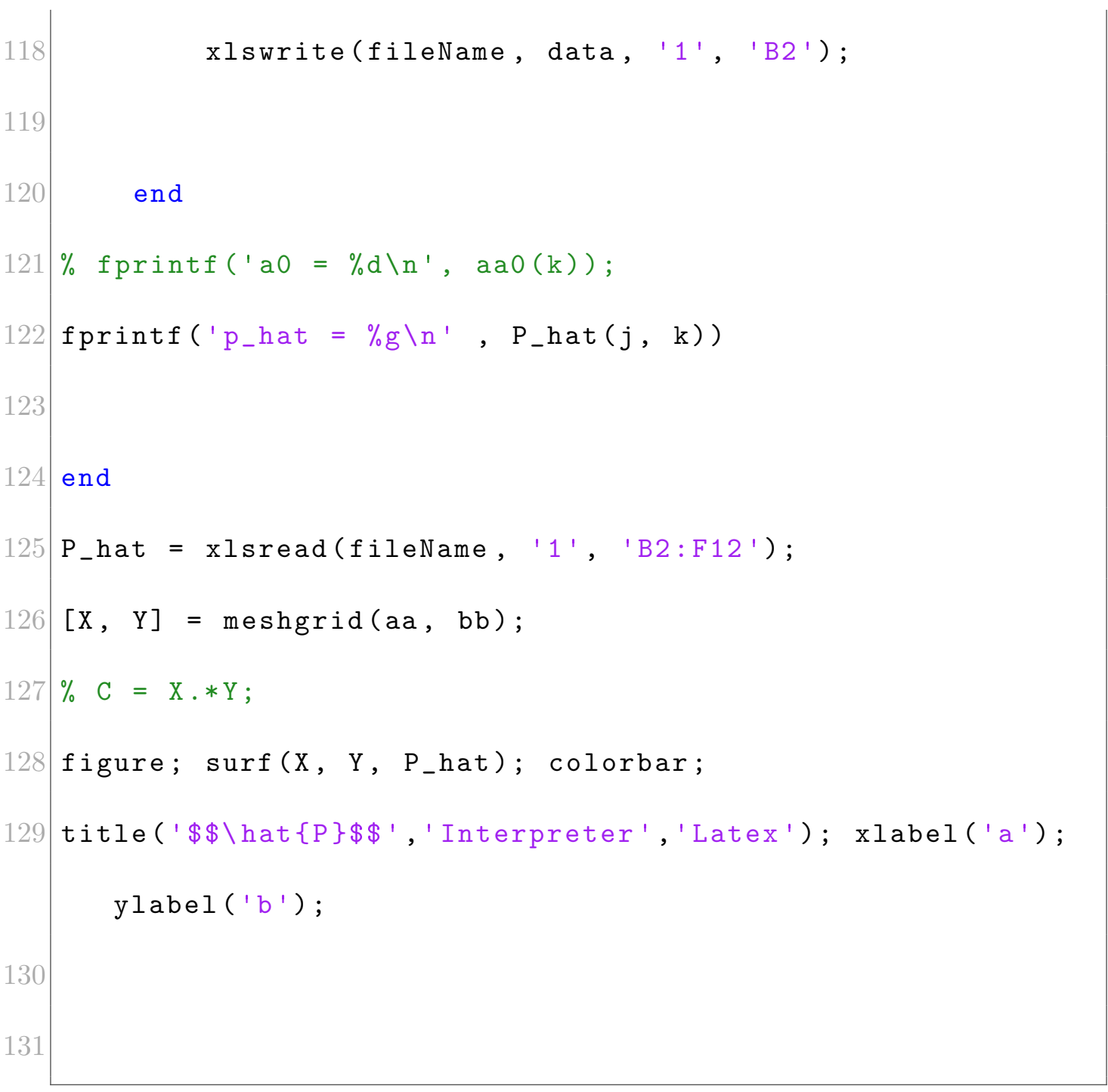

